\title{
A System Dynamic Transmission Model (SYStrans) to Simulate Epidemic Dengue Environment
}

\author{
Rifat Anwar
}

Follow this and additional works at: https://researchrepository.wvu.edu/etd

\section{Recommended Citation}

Anwar, Rifat, "A System Dynamic Transmission Model (SYStrans) to Simulate Epidemic Dengue Environment" (2017). Graduate Theses, Dissertations, and Problem Reports. 5116.

https://researchrepository.wvu.edu/etd/5116

This Thesis is protected by copyright and/or related rights. It has been brought to you by the The Research Repository @ WVU with permission from the rights-holder(s). You are free to use this Thesis in any way that is permitted by the copyright and related rights legislation that applies to your use. For other uses you must obtain permission from the rights-holder(s) directly, unless additional rights are indicated by a Creative Commons license in the record and/ or on the work itself. This Thesis has been accepted for inclusion in WVU Graduate Theses, Dissertations, and Problem Reports collection by an authorized administrator of The Research Repository @ WVU. For more information, please contact researchrepository@mail.wvu.edu. 


\title{
A System Dynamic Transmission Model (SYStrans) to Simulate Epidemic Dengue Environment
}

\author{
Rifat Anwar \\ Thesis submitted to the \\ College of Engineering and Mineral Resources \\ at West Virginia University \\ in partial fulfillment of the requirement for the degree of \\ Master of Science in \\ Civil and Environmental Engineering
}

\author{
Antar Jutla, PhD., Chair \\ Radhey S. Sharma, PhD., Co-Chair \\ Lian-Shin Lin, PhD. \\ Department of Civil and Environmental Engineering \\ Morgantown, West Virginia \\ 2017
}

Keywords: system dynamic, SYStrans, dengue environment, epidemic (๖) 2017 Rifat Anwar 


\section{ABSTRACT \\ A System Dynamic Transmission Model (SYStrans) to Simulate Epidemic Dengue Environment \\ Rifat Anwar}

Dengue is the most significant arthropod-borne virus in terms of human morbidity and mortality. Geographic expansion of dengue and intensity of outbreak has amplified significantly during the last few decades. Thus, the understanding of the dynamic of the large outbreaks has become indispensable for planning of control interventions in future epidemics. In this regard, local entomological, meteorological and epidemiological parameters based dengue models can be an essential tool for better interpretation of dengue-climate relationship at a regional scale. Process based modelling is resourceful in combining the vector and host dynamic along with the response to the meteorological factors for dengue transmission. In previous studies, process based models have not dealt with the integrated impact of vector-host dynamic and dengue transmission epidemiology by incorporating weather dependent transmission mechanism. In this study, a processbased model has been developed and validated for Iquitos of Peru, based on both vector and host population dynamic as well as the whole infection transmission mechanism. The sole objective was to develop a simple model to represent the actual scenario triggering dengue epidemic considering the most important features of vector population dynamics, transmission mechanism and environmental linkages. The model has used remote sensing or satellite based environmental data and also introduced dew point temperature as a new and effective weather parameter to depict the transmission process of dengue. The model has been capable of simulating the peak and moderate scenario in temporal scale, with considerable quantification of the actual number of cases for the 2004 and 2008 epidemics. Eventually, this type of model can be modified to use for different regions to predict the peak scenario based on local weather parameters effecting the infection transmission and vector development process along with population density. 


\section{Acknowledgements}

I would like to convey my deepest gratitude to my thesis supervisor, Dr. Antar Jutla. I am grateful to him for inspiring me through the path of my journey in acquiring knowledge and supporting me to develop the thirst for research. I thank him for his immense guidance and support. I am thankful to my committee members, Dr. Radhey S. Sharma and Dr. Lian-Shin Lin for their encouragement and knowledge that has guided me in improving my research. Their course works helped me incalculably during my research period. My deepest gratitude to my father, Nurul Anwar and my mother, Parveen Fatema, for whom I have achieved every success in my life. Their unconditional love and support has been by biggest strength. I would like to thank my beloved husband, Musfique Ahmed, for his unconditional support and love and for always being by my side. I would like to express my gratitude to him, for without his support I would not be able to achieve any success. I would like to thank my father in law, Md Jasim Uddin Ahmed and mother in law, Maksuda Begum, for their immense love and support. I would like to express my gratitude to my sisters and sister in law, for always being there for me. I express my greatest gratitude to my creator, Allah, for giving me the strength and being the best planner of my life. 


\section{Contents}

Acknowledgements ....................................................................................................................iii

List of Figures............................................................................................................................. V

List of Tables .......................................................................................................................... vii

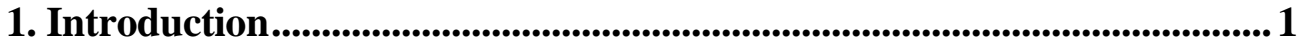

1.1 Background ....................................................................................................

1.2 Outbreak Locations .............................................................................................

1.3 Study Area.....................................................................................................

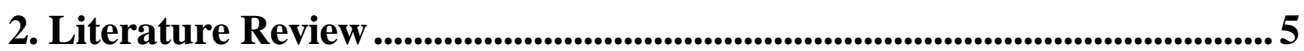

2.1 Literature Review on Dengue Modelling.............................................................

2.2 Literature Review on Climatic Parameters...........................................................10

3. Methodology ………........................................................................................................ 11

3.1 Background on Model Set-up ................................................................................11

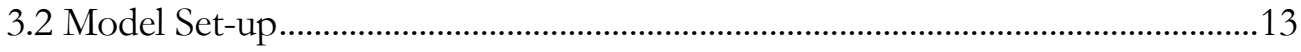

4. Data............................................................................................................................................ 17

5. Model Optimization and Calibration....................................................................... 19

6. Results and Simulations .............................................................................................. 22

7. Conclusion ..................................................................................................................... 26

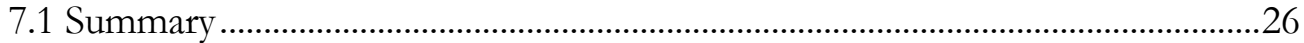

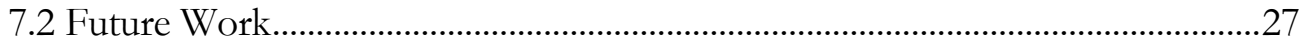

Appendix ...................................................................................................... 28

Bibliography ............................................................................................................ 75 


\section{List of Figures}

Figure 1: Dengue Hotspot Locations .............................................................................

Figure 2: Classification of Hotspot Countries ................................................................

Figure 3: Study Area..............................................................................................

Figure 4: Literature Review on dengue modeling ..............................................................

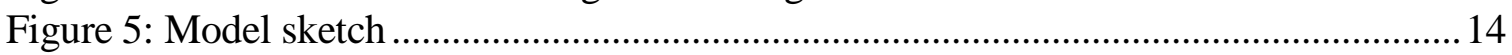

Figure 6: Model sketch in simulated form ................................................................... 14

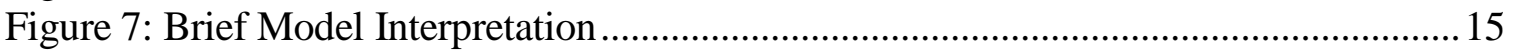

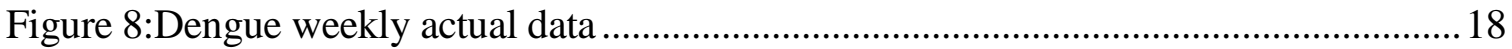

Figure 9: Sensitivity graph for all parameters...……………………………………...2 20

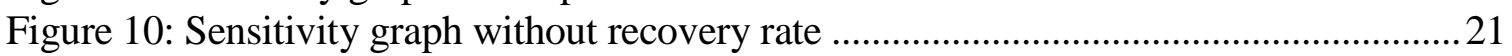

Figure 11: Model simulated and actual cases for 2002 (Model Calibration).........................2

Figure 12: Model simulated and actual cases for 2008 (Validation) .....................................2 24

Figure 13: Model simulated and actual cases for 2004 (Validation) .....................................24

Figure 14: Probability of exceedance of actual and medeled cases (2008) ………...............25

Figure 15: Probability of exceedance of actual and modeled cases (2004) ..........................2.

Figure 16: Vector life cycle model interpretation (part 1)...................................................2. 28

Figure 17: Vector life cycle model interpretation (part 2)....................................................29

Figure 18: Transmission and population model interpretation ..............................................30

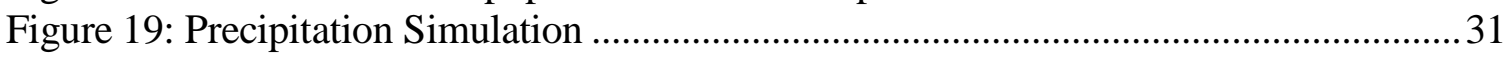

Figure 20: Precipitation index simulation ........................................................................

Figure 21: Precipitation induced container simulation .......................................................... 32

Figure 22: Habitable water simulation .............................................................................. 32

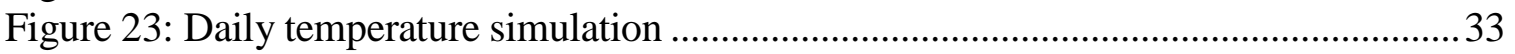

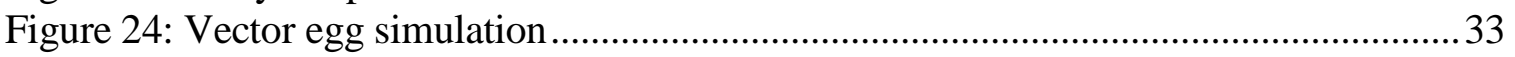

Figure 25: Egg development rate simulation ................................................................... 34

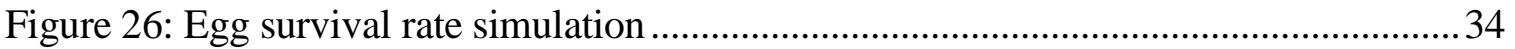

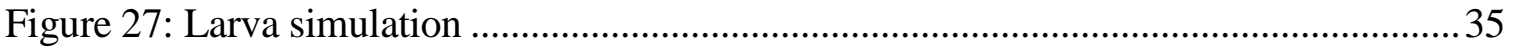

Figure 28: Larva development rate simulation ....................................................................35

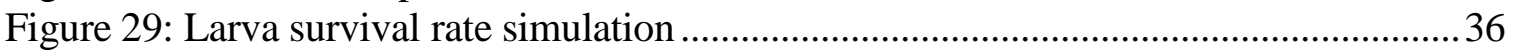

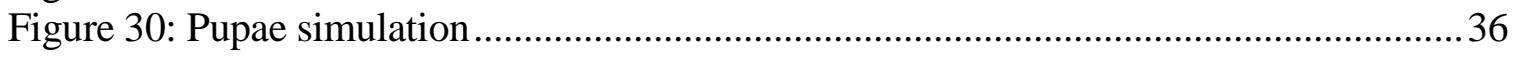

Figure 31: Pupae development rate simulation..................................................................... 37

Figure 32: Pupae survival rate simulation....................................................................... 37

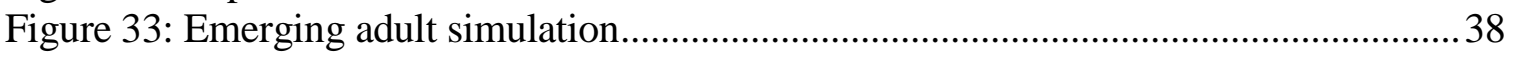

Figure 34: Female adult simulation................................................................................... 38

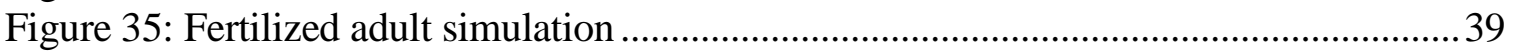

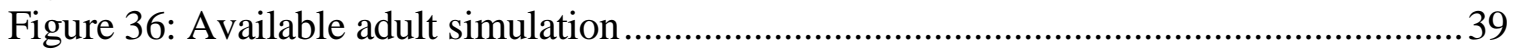

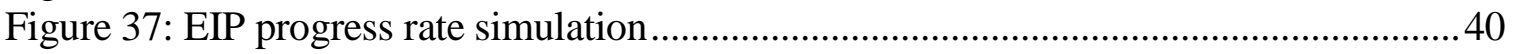

Figure 38: Vector infection probability simulation ..............................................................4

Figure 39: Dew point temperature based persistency factor simulation .................................4 41

Figure 40: Adult biting rate simulation ........................................................................ 41

Figure 41: Infectious adult simulation .......................................................................4 42

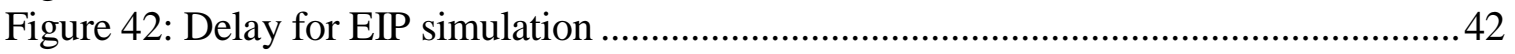

Figure 43: Effective infectious adult simulation .................................................................. 43 


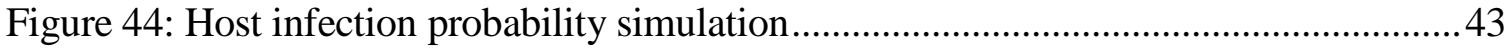

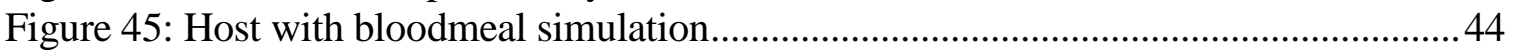

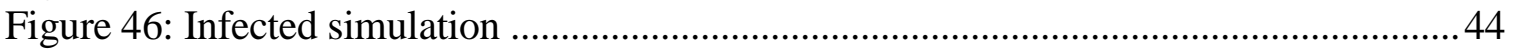

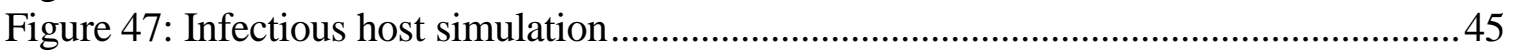

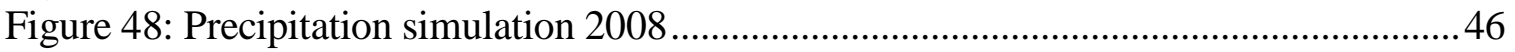

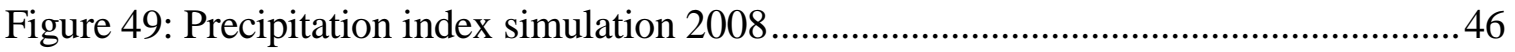

Figure 50: Precipitation induced container simulation 2008 .............................................47

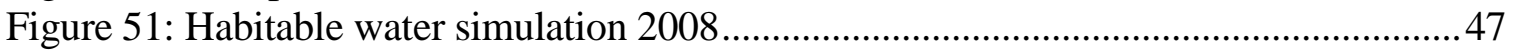

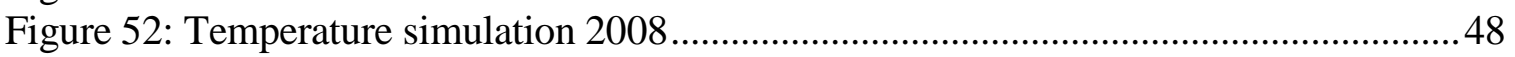

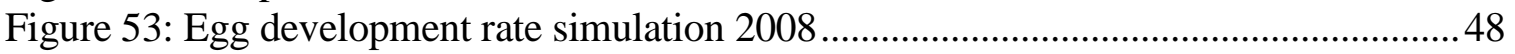

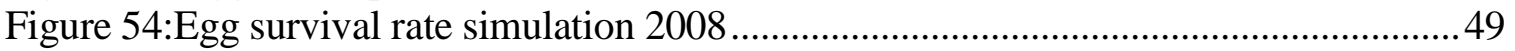

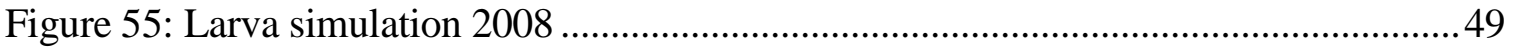

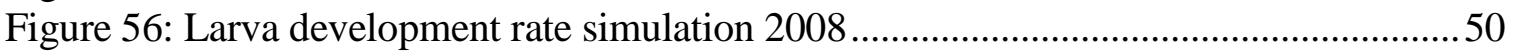

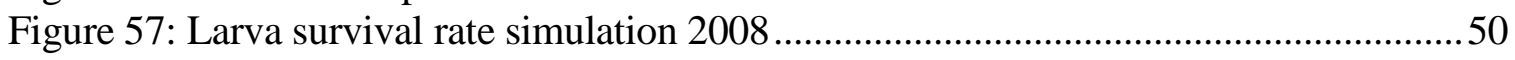

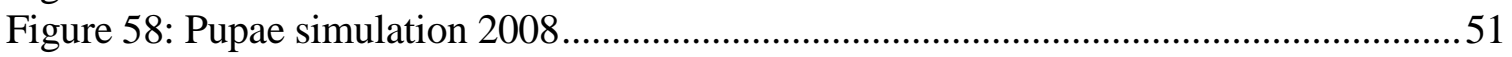

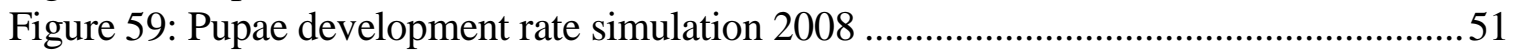

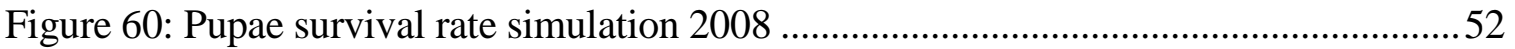

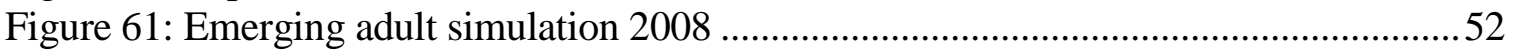

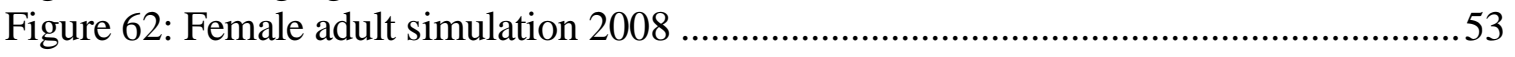

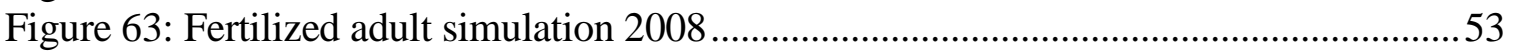

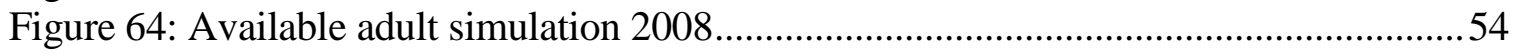

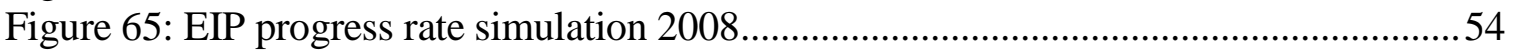

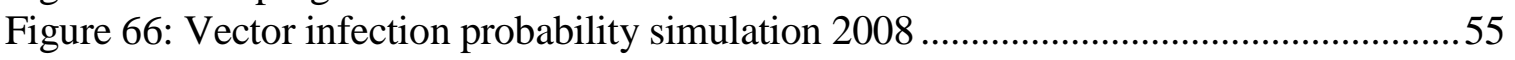

Figure 67: Dew point temperature based persistency factor simulation 2008 .....................55

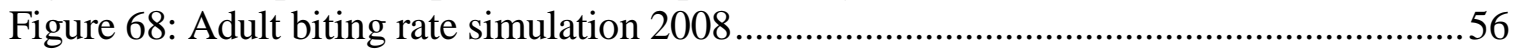

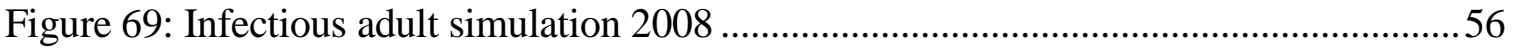

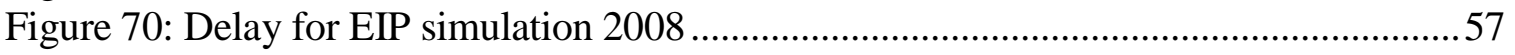

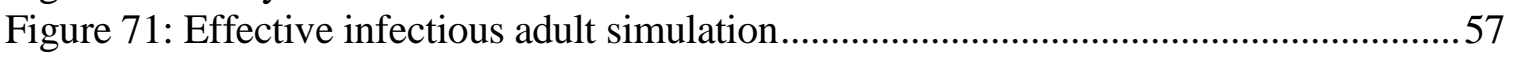

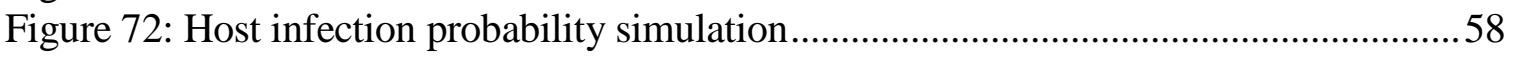

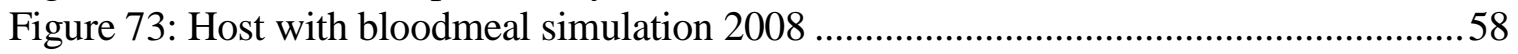

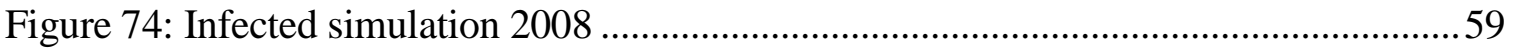

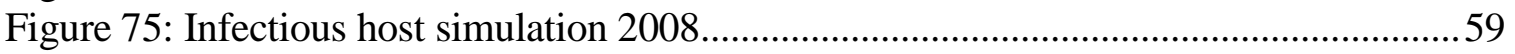

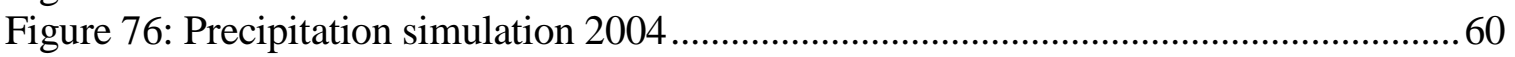

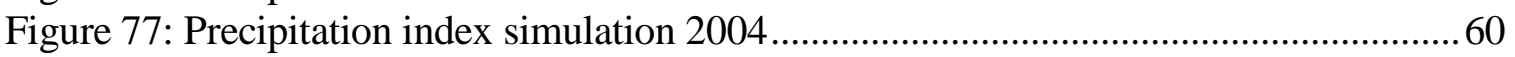

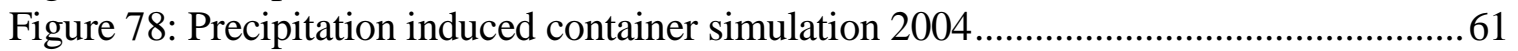

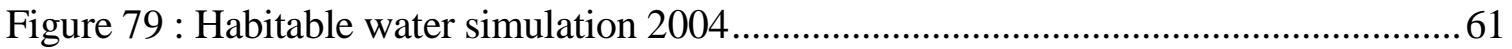

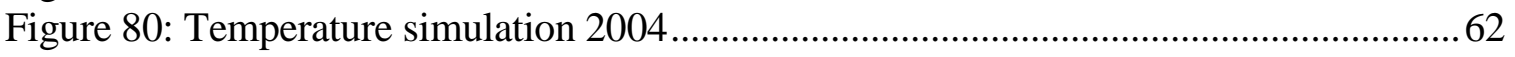

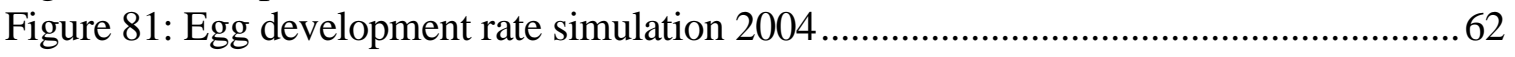

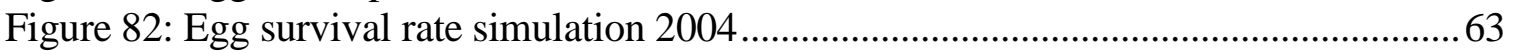

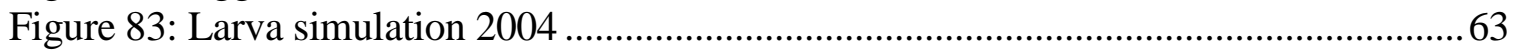

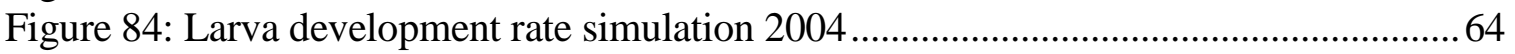

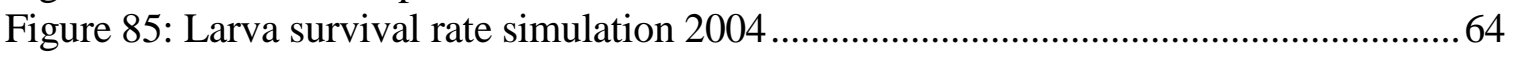

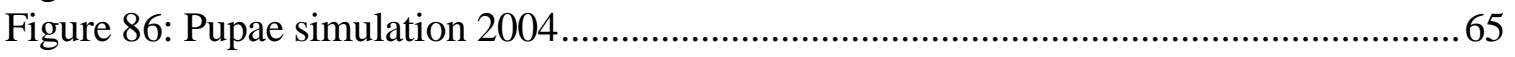

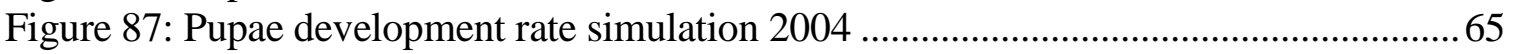

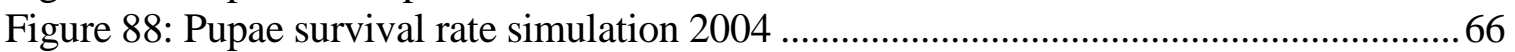




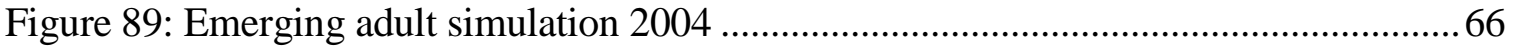

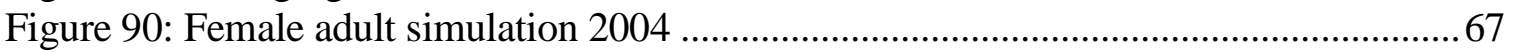

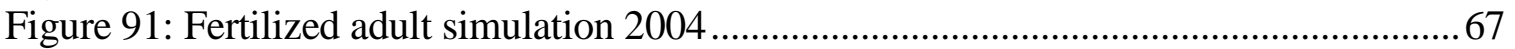

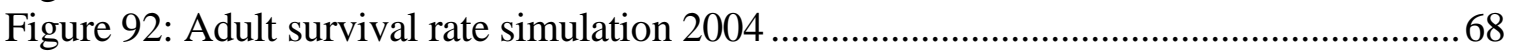

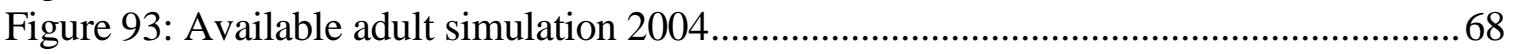

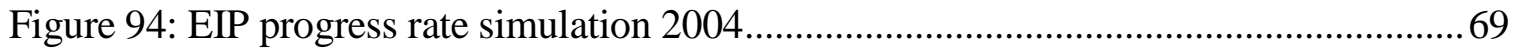

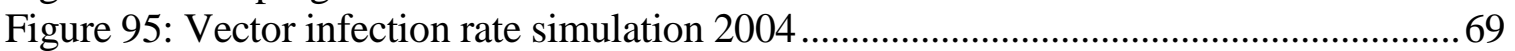

Figure 96: Dew point temperature based persistency factor simulation 2004 ..................... 70

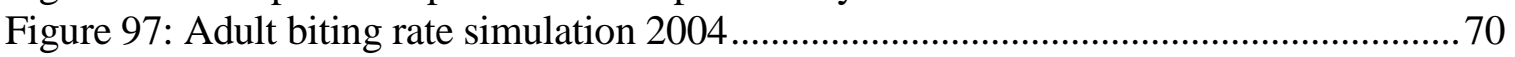

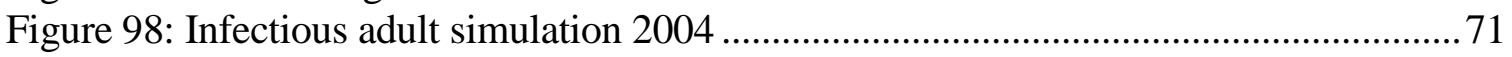

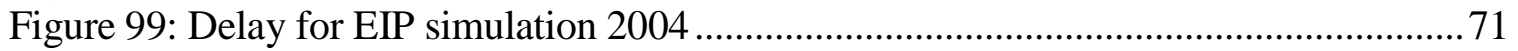

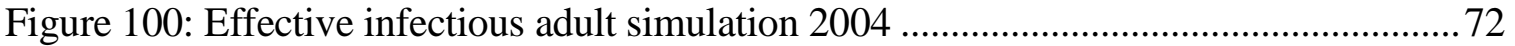

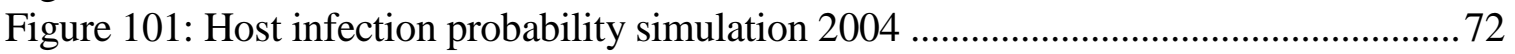

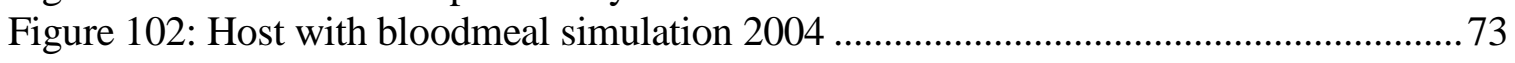

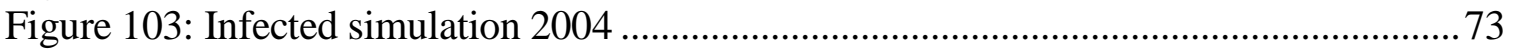

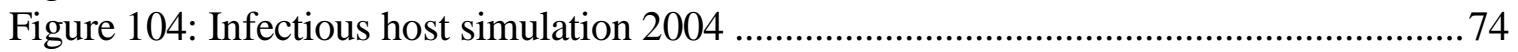

\section{List of Tables}

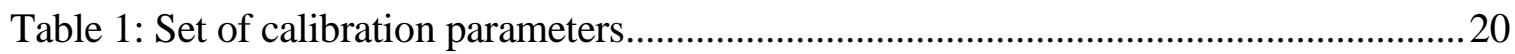




\section{Introduction}

\subsection{Background}

Dengue is the most significant arthropod-borne virus in terms of human morbidity and mortality (DJ, 2002) (SB, 2008). Geographic expansion of dengue has intensively amplified since the last few decades (Morrison, et al., 2010). Whereas, higher urban population density and availability of vector habitats have intensified the transmission with increasing number of cases (Karl, Halder, Kelso, Ritchie, \& Milne, 2014). Thus, the understanding of the dynamic of the large outbreaks has become indispensable for planning of control interventions in future epidemics (Anderson \& May, 1992). Aedes aegypti is considered as the main vector for dengue transmission, which is the most efficient vector for abroviruses as it is anthropophilic and flourish in close proximity to humans (Otero \& Solari, 2010). However, vector population and vector competence of Aedes varies significantly with seasonal environmental condition (Mohammed \& Chadee, 2011).

Climatic condition and variability play a significant role in regulating entomological process of vectors and epidemiology of vector borne diseases like dengue (Githeko, et al., 2000). The evolving and survivor factors of Aedes are temperature dependent and there is a favorable threshold range of temperature supporting the development at each stage (Hopp \& Foley, 2001). The ecology of virus transmission and replication is also temperature dependent, which regulates extrinsic incubation period (EIP), gonotrophic cycle, biting rate, life span as well as human response or interaction (FOCKS, et al., 2000) (Barbazan, et al., 2010) (Mohammed \& Chadee, 2011). On the other hand, precipitation pattern has been associated to the availability of aquatic breeding sites, while few studies discussed about the opposite impact of precipitation on vector availability (Althouse, et al., 2015) (Shope, 1991) (Kearney, et al., 2009) (O'Gower, 1956) (Olson, 2006). The effect of other environmental variables has been skeptical and utilize differently in different studies.

Local entomological, meteorological and epidemiological parameters based dengue models can be an essential tool for better interpretation of dengue-climate 
relationship at a regional scale. Vector-host transmission modelling, representing both vector and host dynamic, is essential to evaluate the effectiveness of different intervention activities (Andraud, et al., 2012). In addition, deterministic and process-based modelling facilitates in analyzing the regional ecology of dengue-climate relations and thus pivotal to adopt regional control strategies (Bannister-Tyrrell, et al., 2013). Different deterministic models have been developed focusing on different aspects including variable human population, variable vector population, vertical and mechanical transmission and presence of different strains (Otero \& Solari, 2010).

Process based modelling is resourceful in combining the vector and host dynamic along with the response to the meteorological factors for dengue transmission (Vezzani, et al., 2004). This dynamic modelling process can incorporate the biophysical relationship between different entomological and environmental factors (Vaidya, et al., 2014). In previous studies, process based models have not dealt with the integrated impact of vector-host dynamic and dengue transmission epidemiology by incorporating weather dependent total transmission mechanism (Morin, et al., 2013). In this study, a processbased model has been developed for Iquitos of Peru, based on both vector and host population dynamic as well as the whole infection transmission mechanism. The response of entomological activities and transmission dynamic to the daily local weather parameters has been incorporated to the model to represent the local transmission scenario. Aedes aegypti based vector life cycle model is integrated with SEIR based population model and a conceptual transmission model developed by replicating the infection transmission process between vector and host. The study has proposed the use of remote sensing or satellite data in process-based modelling. The potential of remote sensing data has inflated recently for the study of diseases related to environmental condition as it provides information about weather or environmental variability on different spatial and temporal scale (Side \& Noorani, 2013). The study also introduces dew point temperature as a new and effective weather parameter to depict the transmission process of dengue. Eventually, this type of model can be modified to use for different regions to predict the peak scenario based on local weather parameters effecting the infection transmission and vector development process along with population density. 


\subsection{Outbreak Locations}

As a background study, a detail literature review has been conducted on identifying historical hotspots of dengue epidemic. Annual large outbreaks or epidemic scenarios has been reviewed to obtain country wise specific locations on city level. Figure 1 shows locations of dengue outbreaks, where mainly epidemic scenarios has been included for the period of 1960 to 2016. For African regions outbreaks has been suggested to be less documented in literatures, where the number of locations is supposed to be more in terms of risk and vulnerability. The hotspot countries have been classified based on quantitative historical evidence of large outbreaks (Figure 2). Countries with less than five large historical outbreaks have been identified as low-risk countries, whereas countries have been demonstrated to be at medium-risk where large outbreaks occured between five to ten times (Figure 2). Countries with more than ten outbreaks have been stated as high-risk countries (Figure 2). This background study facilitated in selecting the study area based on vulnerability and depicts the overall global pattern of dengue outbreak.

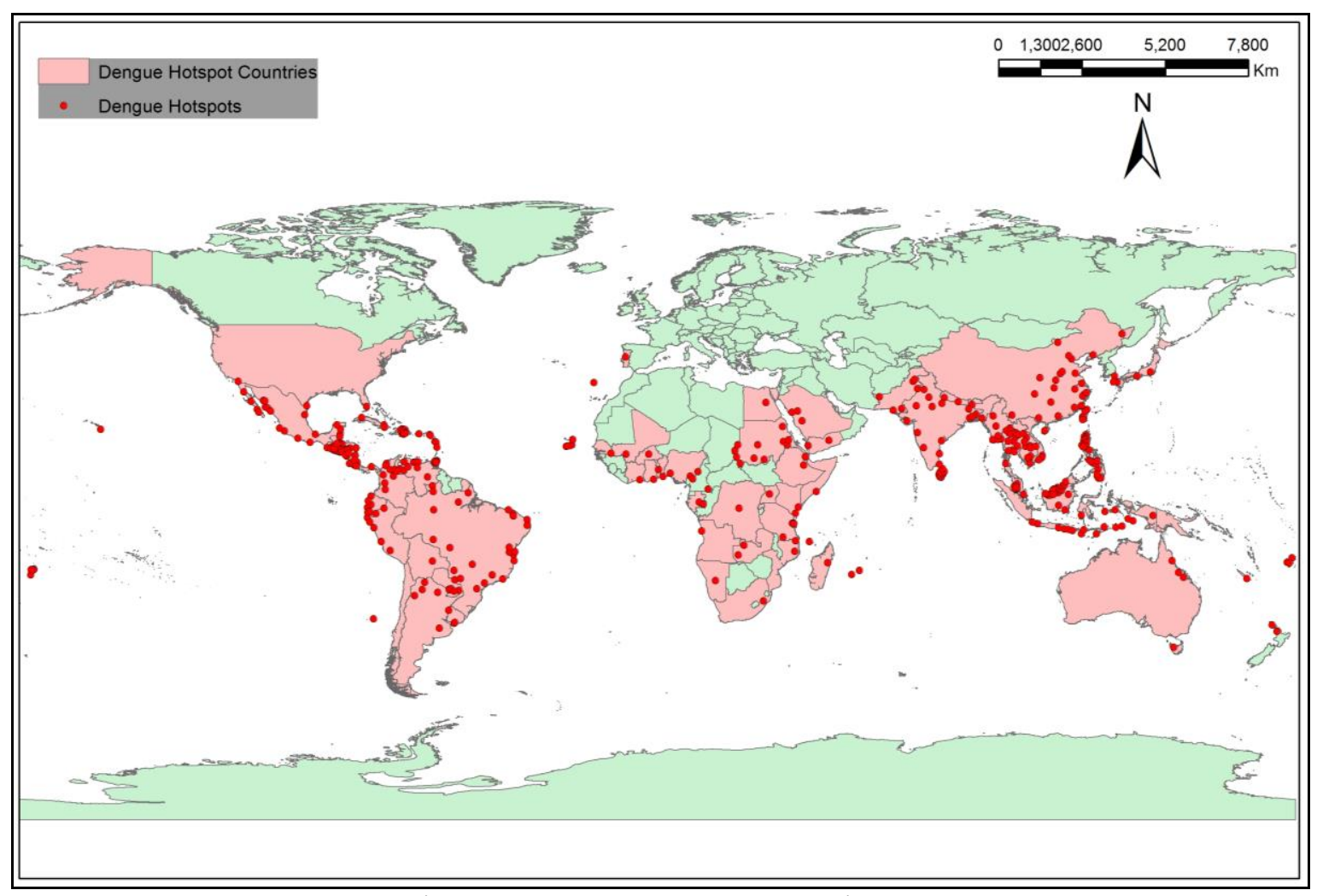

Figure 1: Dengue Hotspot Locations 


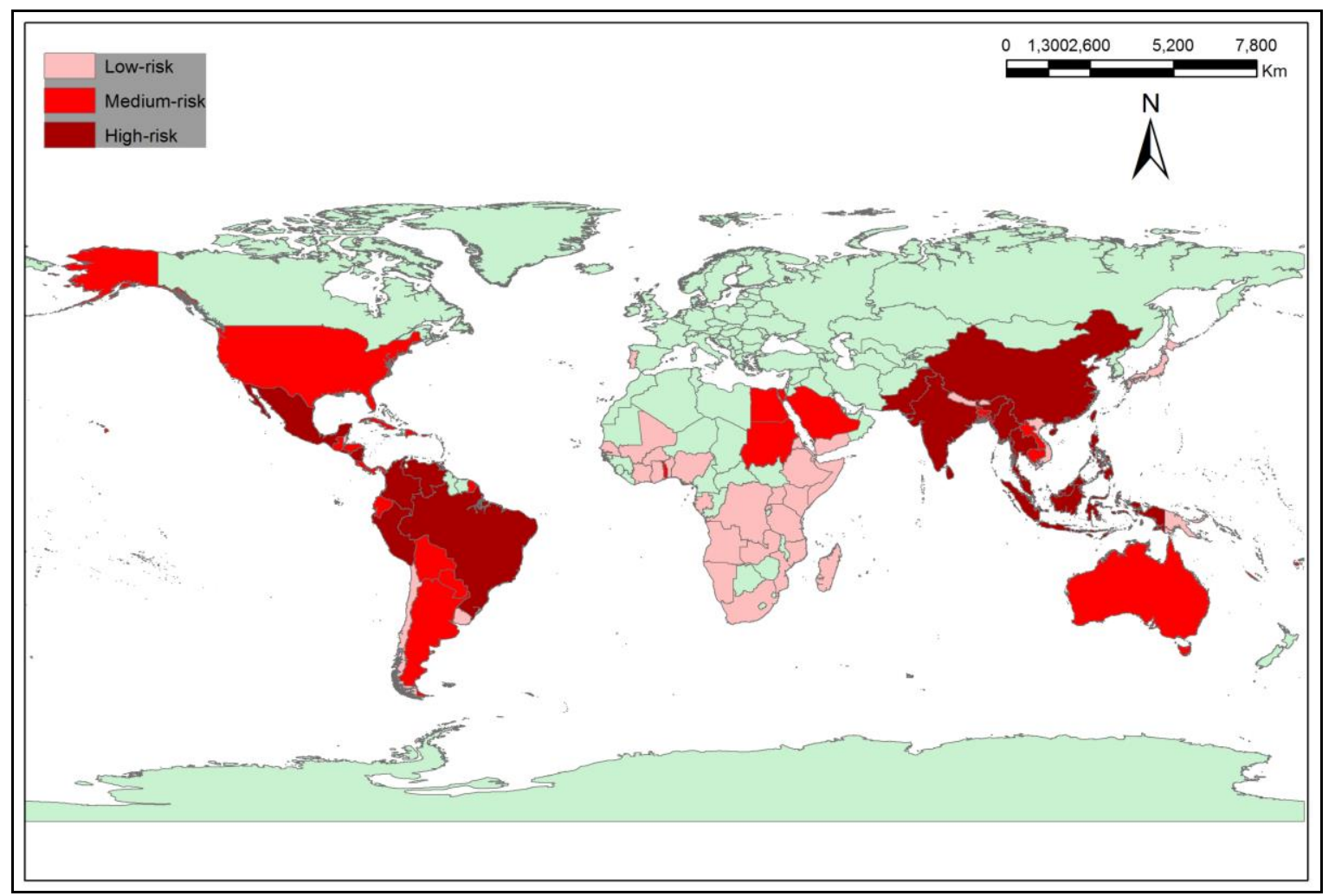

Figure 2: Classification of Hotspot Countries

\subsection{Study Area}

Our model has been developed for Iquitos, an isolated city in the Amazonian region of Peru having around 400,000 people (Figure 3). Peru has been identified as a country with high dengue risk (Figure 2). The city of Iquitos has well documented history of dengue virus transmission with recent and dramatic outbreaks. The climate of the city is tropical, with precipitation occurring throughout the year (Morrison, et al., 2010). In Iquitos, dengue serotype DEN-1 was first identified in 1990, where American genotype DEN-2 invaded during the large outbreak of 1995-1996 (Chowell, et al., 2008). However, the cocirculation of all four serotypes started during the epidemic of 2000-2001 (Montoya, et al., 2003). 


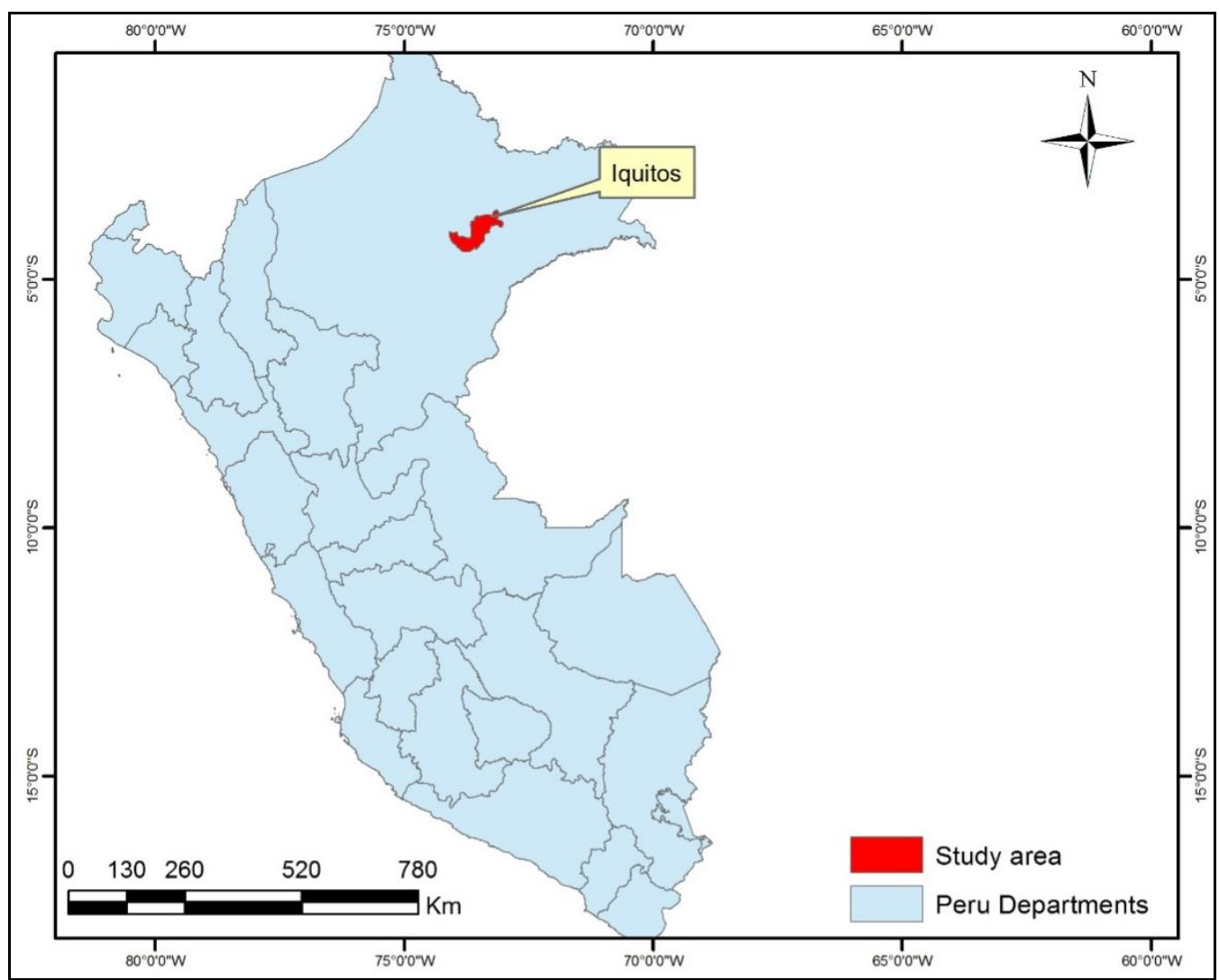

Figure 3: Study Area

\section{Literature Review}

\subsection{Literature Review on Dengue Modelling}

The dynamics of dengue epidemic has been modelled in different literatures to perceive the ideal outbreak scenario as these models can exemplify the disease dynamic and eventually aid in disease control (Diekmann \& Heesterbeek, 2000) (Esteva \& Vargas, 1998). The development of these models require intensive knowledge on relationship between different entomological and epidemiological variables (Ljung, 1999) (Massad, et al., 2003). However, unreported and misdiagnosed cases is a source of uncertainty in the model (World Health Organization, 2009), whereas understanding the relationship and prominence of different environmental factors and disease epidemic has been observed to be the prime challenge. The influence or impact of environmental factors can vary spatially due to socio-economical condition and life style (Lozano et al). Also, the lag between 
dengue prevalence and these environmental factors has been identified to be different for models developed for different locations (Yu, et al., 2011).

A generalized mixed model (GLMM) approach was followed by Lowe et al, where Bayesian framework was used for epidemic forecasting. The model quality has been assessed based on the ability to depict the warning of the peak dengue season (Lowe, et al., 2011). Torres et al used fuzzy model identification technique, where multiresolution analysis has been used to replicate original dengue and severe dengue epidemic in Colombia (Torres, et al., 2014). Buczac et al used fuzzy association rule models to identify spatial risk level of dengue incidence in terms of environmental and social condition of prior outbreaks (Buczak, et al., 2012). Yu et al proposed spatio-temporal prediction approach for dengue, using stochastic Bayesian Maximum Entropy (BME) analysis (Yu, et al., 2011). Studies developed mathematical and statistical models (deterministic or stochastic) to illustrate spatial or spatio-temporal epidemic pattern in terms of interaction between vectors and their human-hosts (Nishiura, 2006) (Otero, et al., 2006) (Maidana \& Yang, 2008).

Time-series regression approach has been used for predicting dengue fever using different weather variables in many literatures (Depradine \& Lovell, 2004) (Wu, et al., 2007) (Luz, et al., 2008) (Brunkard, et al., 2008) (Chakravarti \& Kumaria, 2005). Lu et al predicted dengue incidence for China using one-month prior minimum temperature, minimum humidity and wind velocity as the major predictors (Lu, et al., 2009). Brunkard et al and Diaz et al developed weather variable based autoregressive model to predict weekly changes in dengue incidence with changing weather pattern (Brunkard, et al., 2008) (Hurtado-Diaz, et al., 2007). Autoregressive integrated moving average (ARIMA) models have been fitted with time series of dengue fever incidence in few literatures $(\mathrm{Wu}$, et al., 2007) (Hu, et al., 2010). Chan et al developed historical case based regression model using environmental condition and population density to provide risk prediction for small areas (Chan, et al., 2015). Regression models have been mainly used to assess cross correlations between environmental variables and dengue fever cases with different lags (Fuller, et al., 2009). Regression models have been also explored to evaluate weather dependent vector abundance and their impact on dengue outbreak (Azil, et al., 2010). Some studies also 
developed transmission potential models for dengue, with major focus on temperature dependent transmission capacity of the vector population (Barbazan, et al., 2010). These studies used historical dengue cases for risk prediction and most of these models utilized temperature, precipitation and humidity to forecast the dengue fever cases (Wu, et al., 2007) (Hu, et al., 2010).

Ecological niche modelling has been also used to predict the risk zone of dengue occurrence based on satellite environmental data and history of dengue fever cases. Arboleda et al conducted ecological niche modelling with satellite environmental data and dengue cases (Arboleda, et al., 2009). Peterson et al used ecological niche modelling to predict monthly distribution, dynamics and activity of dengue vector (Peterson, et al., 2005). The geographical expansion of the risk of dengue fever transmission has been assessed by Hales et al, where geographical distribution has been modelled based on vapor pressure (Hales, et al., 2002). Studies have conducted tele-epidemiology based analysis to map entomological risk of vector distribution around dwellings (Machault, et al., 2014). Climate-driven statistical and process based models have been developed to assess the climate change impact on global distribution of dengue (Morin, et al., 2013). These type of models mainly evaluated the geographic distribution of the risk of dengue and have not focused on spatio-temporal risk pattern (Arboleda, et al., 2009).

Remote sensing data has been used for understanding the spatio-temporal risk of dengue and modelling the global distribution of the disease due to promising scope of this higher spatial resolution data (Anno, et al., 2015). Remote sensing data has gradually become very efficacious for epidemiological studies due to prominent development in providing environmental data and landuse pattern (Curran, et al., 2000) (Hay, et al., 2010). Remote sensing or satellite data has been effectively used in modelling vector borne diseases (Stefani, et al., 2013) (Yang, et al., 2005) (Kalluri, et al., 2007) (Bergquist, 2001), including distribution of dengue vector (Neteler, et al., 2011) (Roiz, et al., 2011) (Estallo, et al., 2008) (Fuller, et al., 2009) (Vanwambeke, et al., 2011) (Sarfraz, et al., 2012) as well as human dengue cases (Benthem, et al., 2005) (Rotela, et al., 2007). These studies focused on mapping vector habitats, evaluating environmental factors influencing vectorial entomology and epidemiological risk of disease transmission (Rotela, et al., 2007). In these 
studies, temperature, rainfall and humidity were the most commonly used satellite based weather variables along with vegetation index and land cover pattern. Some studies have also used wind speed and digital elevation model derived drainage pattern in the risk modelling (Khalid \& Ghaffar, 2015). Many studies utilized the normalized difference vegetation index (NDVI) for associating the changing land use pattern with the prevalence of dengue (Rotela, et al., 2007) (Troyo, et al., 2009). MODIS and Landsat data have been mostly used, whereas some studies have also used AVHRR, SPOT and QuickBird satellite data. Remote sensing data can be more expedient for modelling of infectious diseases like dengue, compared to the station based data (Tran \& Raffy, 2006). Recently, process based or network based modelling has emerged as a proficient tool for studying geotemporal epidemics of infectious diseases (Newman, 2002). However, their practical simulation has not been adapted with use of realistic remotely sensed data (Tran \& Raffy, 2006).

Studies used SEIR (susceptible-exposed-Infective-resistant) models to study vector borne diseases like dengue (Murray, 2003). These type of compartmental models have been majorly used to assess the host dynamics (Pongsumpun \& Tang, 2001), influence of vector control strategies (Newton \& Reiter, 1992) and competition between different strains (Feng \& Velasco-Herna'ndez, 1997). However, these models have not considered combined dynamics of the mosquito life cycle and development as well as the infection transmission process to represent the actual scenario. Modelling vector distribution can portray the risk pattern of dengue, however is not sufficient to replicate the actual scenario with the transmission dynamics (Machault, et al., 2014). On the other hand, modelling population dynamic cannot determine the effect of vector development and transmission activity, thus combination of the whole process is necessary to illustrate the real scenario.

A detail literature review has been conducted to evaluate the previous studies on dengue modelling (Figure 4). Figure 4 shows that only few studies used remote sensing data, while mathematical modelling based studies have not explored the field of remote sensing or satellite data. There are some commonly used environmental parameters. Most of the studies based on mathematical modelling approach have not evaluated the combined 
impact of the environmental variables on vector development, host dynamic and vectorhost transmission process.
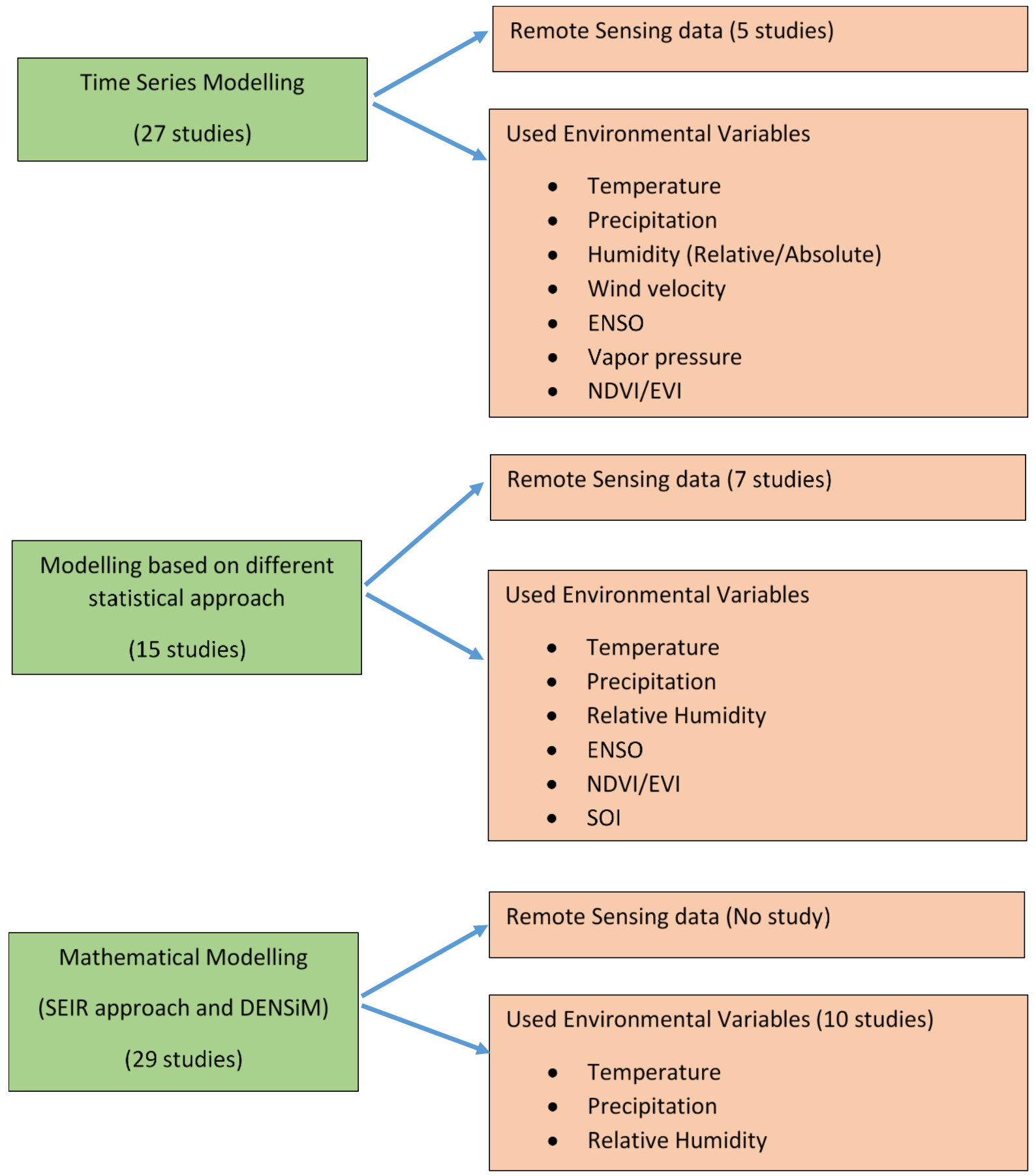

Figure 4: Literature Review on dengue modeling 


\subsection{Literature Review on Climatic Parameters}

Climatic condition and variability play a significant role in regulating entomological process of vectors and epidemiology of vector borne diseases. Different literatures discussed that vector-host distribution as well as vector-host-virus complex interaction influences prevalence of dengue epidemic (Althouse, et al., 2015). The intricate relationship is related to seasonal climatic condition as vector abundance, competence and virus transmissibility is significantly climate regulated (Mohammed \& Chadee, 2011) (Focks, et al., 2000). In most of the studies, temperature and precipitation have been recognized as the major climatic factors, linking dengue prevalence and climate around the endemic areas (Descloux, et al., 2012) (Dom, et al., 2012). Many studies discussed and reviewed the significant influence of temperature on faster viral replication rate within vector as well as intensified transmission scenario (Morin, et al., 2013). Precipitation and temperature has been observed to be the most commonly used climatic factors for prediction of dengue epidemic and developing risk maps (Sang, et al., 2014) (Yu, et al., 2011) (Chan, et al., 2015). Also, relative humidity has been perceived as a significant climatic factor along with temperature and precipitation in different studies (Descloux, et al., 2012) (Dom, et al., 2012) (Duncombe, et al., 2013). Hales et al conferred expedition of transmission efficiency with increasing temperature and relative humidity effecting epidemiological activities of vector like extrinsic incubation period (EIP) and gonotrophic cycle (Hales, et al., 2002). Costa et al discussed the influence of relative humidity in regulating the effect of temperature on vector survival rate, which eventually control transmission efficiency of vector (Costa, et al., 2010). Descloux et al modeled dengue outbreaks in Noumea relating temperature, precipitation, relative humidity, potential evapotranspiration and wind force (Descloux, et al., 2012). Dom et al and Lu et al (Dom, et al., 2012) (Lu, et al., 2009) incorporated temperature, precipitation and relative humidity as climatic parameters for modelling dengue outbreak scenario. Duncombe et al also utilized these parameters for assessing dengue transmission risk through computation of vector density (Duncombe, et al., 2013). Deviation of these parameters (temperature and relative humidity) has been also considered as predictive factors in studies (Wu, et al., 2007). Studies also reconnoitered the association of El Niño southern oscillation (ENSO) with 
dengue fever epidemic for different coastal countries (Descloux, et al., 2012). ENSO is acknowledged as an indicator of climate variability, which effects the distribution of dengue vector ( $\mathrm{Hu}$, et al., 2010). Hales et al modelled global distribution of dengue based on vapor pressure as an indicator of humidity (Hales, et al., 2002). Studies also considered drainage pattern and land cover type for predicting dengue outbreak, which are eventually influenced by precipitation pattern by providing or flushing vector breeding sites (Khalid \& Ghaffar, 2015).

Repercussion of different climatic or environmental factors on dengue vector abundance and competence has been observed to be skeptical in different studies (Jansen \& Beebe, 2010). Different studies discussed the dominance of different climatic factors for prevailing outbreak condition of dengue to portray the climate dependent transmission efficiency of vector (Rohani, et al., 2009). Biswas et al analyzed and suggested precipitation and humidity to have more profound impact on vector density compared to temperature as they control larval development and survival (D, et al., 1993). Few studies also suggested the prominence of rainfall over temperature and humidity, whereas dominance of temperature and humidity over precipitation has been conferred in few studies (Anno, et al., 2014) (Favie, et al., 2006). On the other hand, studies speculated lower dengue incidence with higher mean temperature, which contradict with most of the other studies on climate dependent dengue epidemic (Limper, et al., 2014). Araujo et al analyzed association of dengue prevalence with urban heat islands having higher land surface temperature, lower humidity and poor vegetation cover (Araujo, et al., 2015). Also studies identified vegetation dynamics to be a controlling factor for dengue epidemic as it can regulate local moisture supply, evaporation rate and wind speed (Fuller, et al., 2009).

\section{Methodology}

\subsection{Background on Model Set-up}

Mathematical modeling has been used intensively for simulating dengue outbreak, based on the concept of SIR (Susceptible-Infected-Recovered) (Side \& Noorani, 2013). For dengue epidemiology, most of the studies have considered changing host 
population dynamic with constant vector population, while few have considered both host and vector dynamics. On the other hand, few studies have considered the changing vector dynamic with constant population (Esteva \& Vargas, 2000). However, time series modelling has been used for dengue to use long term environmental dataset for predicting dengue cases, where validation of these models with real data have contributed in assessing the significance of different environmental variables. These types of models have been used in many studies with the major focus on environmental data, not considering the population and vector dynamic and concluded with the significance of different variables with different time lag. But, the use of process based simulation model for dengue epidemic has been observed to be limited (Bannister-Tyrrell, et al., 2013). Compared to the statistical models, process based models explicitly explain and illustrate each step of the transmission process as well as the biological life cycle of the vector and thus can be very effective. However, previous studies of process based modelling has not compiled the whole weather influenced infection transmission process with vector and host dynamic models. Moreover, they used station based environmental data and none of them explored the significance of remote sensing data in this regard. They mainly used local temperature and precipitation as environmental variable to simulate local dengue cases (Morin et al, 2013).

In our study, a process based mathematical modelling approach has been used with a system dynamic simulation software named Vensim. It supports continuous simulation and provides graphical modelling interface with interactive tracing of behavior between model variables. Generally process based models provide the advantage of realistically associate different variables and connect them to process the real scenario. This approach is mainly based on theoretical understanding of the whole vector development and dengue transmission process, providing the framework to incorporate the response of environmental conditions to the overall process. It provides easier interpretation of the real scenario with a framework to associate the impact of the different condition on the disease dynamic. In this model, the dynamic or cyclic process is triggered by arrival or introduction of infected people. In our model, we have introduced dew point temperature as a new environmental variable that have not been evaluated before for dengue models. Dew point 
temperature is related to temperature and humidity and higher dew point temperature interpret existence of more moisture in the air and depicts that the air is maximally saturated with water. Temperature and relative humidity has been observed to be the determinant in dengue outbreak occurrence and persistent condition for the outbreak was determined based on number of days with these two variables being in the favorable range (Descloux, et al., 2012). Moreover, studies found absolute humidity to be the most consistent factor for dengue transmission, which elucidate the combined impact of mean temperature and relative humidity on infection proliferation (Xu, et al., 2014). In previous studies, it has been analyzed that the combined effect of temperature and humidity has influence on dengue virus propagation in the vectors (HM, et al., 1998). Thus, we hypothesize that dew point temperature can be a significant factor contributing persistent climatic condition for dengue as it portrays the combined effect of temperature and relative humidity.

\subsection{Model Set-up}

We have studied the spread of the disease by host and vector activities, while considering the effect of different environmental variables on entomological activities of vectors and epidemiology of dengue transmission. The host dynamic has been incorporated based on the traditional approach of susceptible-infected-recovered (SIR), but inspecting the impact of different weather dependent activities before simulating infected host. The motivation of the model is based on combining the vector and host population dynamics, incorporated with weather parameters and delay for fertilization and incubation periods, to simulate realistic temporal and numerical pattern of dengue cases. The model consists of three sub-models (Host Population Dynamic Model, Vector Population Dynamic Model and Dengue Transmission Model), which have been integrated in a way that it represents the whole process (Figure 7). Figure 5 and 6 show the model sketch in normal and simulated form and figure 7 illustrates the schematics of different variables and their interrelationships within different sub models. The model is simulated with daily time step and the model calculates daily vector population at each stage of life depending on survival and development rate related to the daily temperature as well as water availability. 


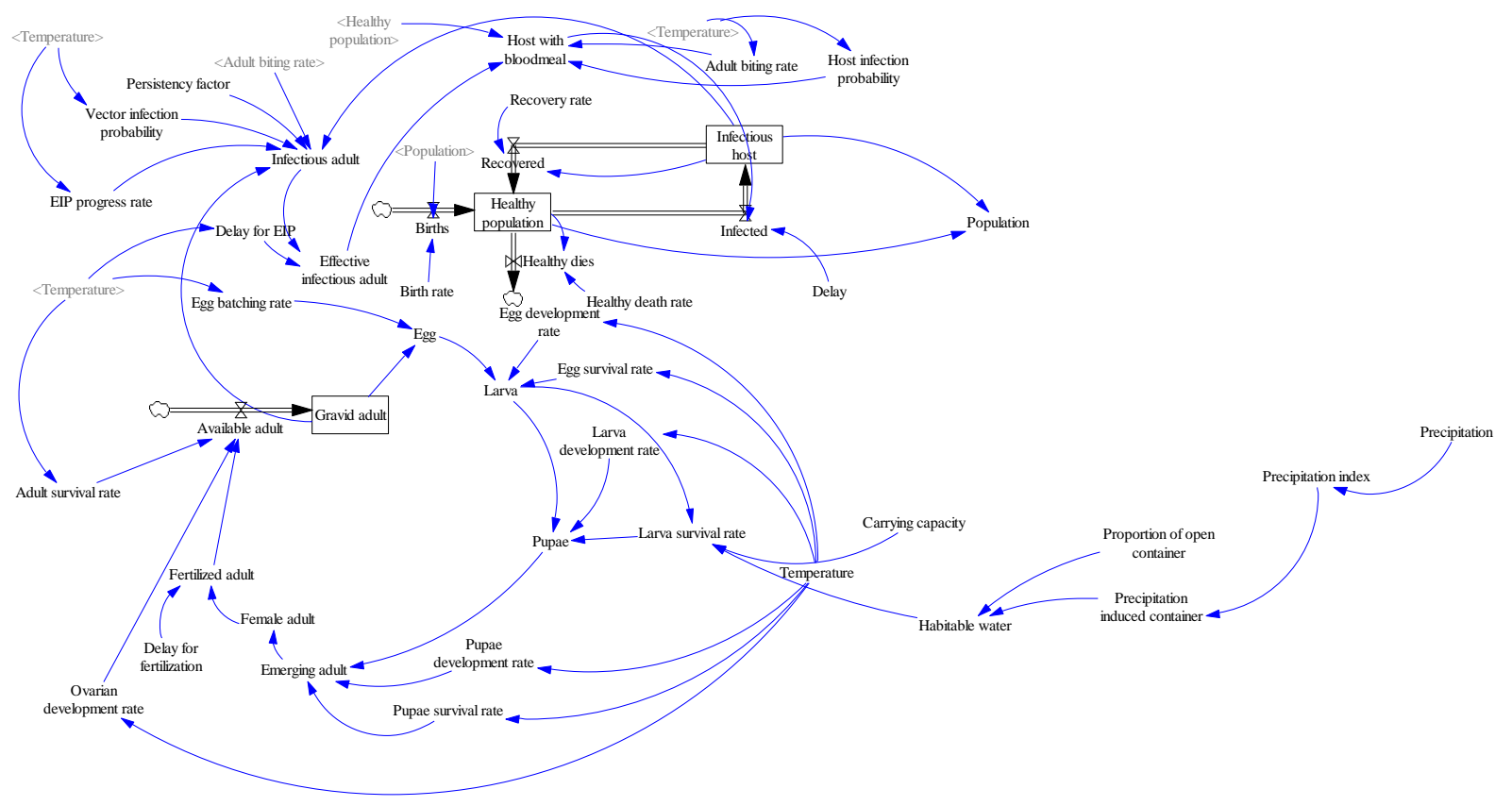

Figure 5: Model sketch

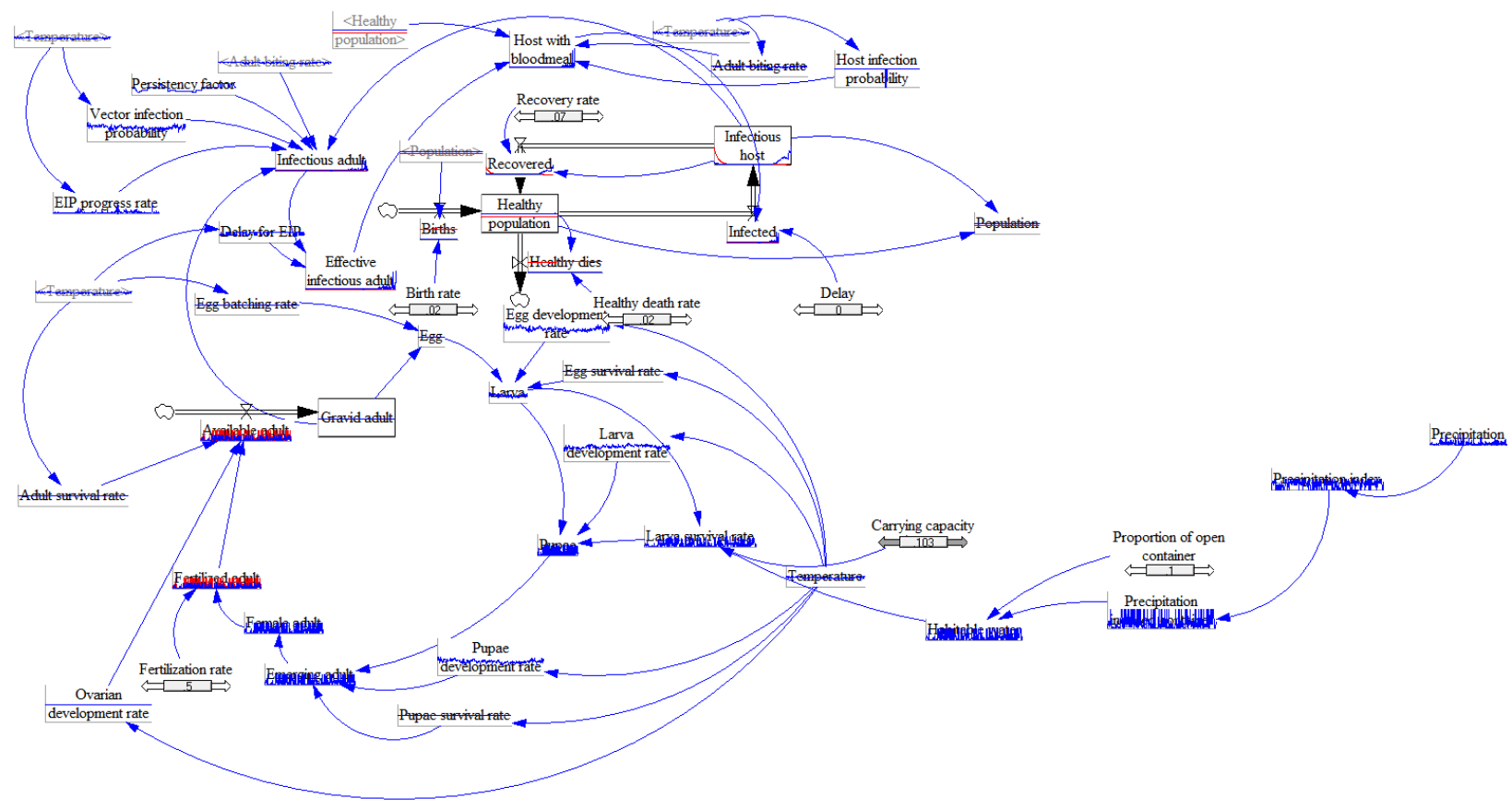

Figure 6: Model sketch in simulated form 


\begin{tabular}{|c|c|}
\hline $\begin{array}{l}\text { Host Population Dynamic } \\
\text { Model }\end{array}$ & $\begin{array}{l}\text { Locationwise healthy population (susceptible host) with birth and death rate associated to the total population (Comprised } \\
\text { of susceptible and infectious host) } \\
\text { Infectious host with few initially infected starting the cycle } \\
\text { Recovered host again joining the susceptible host with a recovery rate }\end{array}$ \\
\hline $\begin{array}{l}\text { Vector Population Dynamic } \\
\text { Model }\end{array}$ & $\begin{array}{l}\text { Vector life cycle started with some available gravid adult providing eggs with temperature dependent egg hatching rate } \\
\text { Life cycle propagates through different stages (larva,pupae,emerging adult) with temperature dependent survival and } \\
\text { development rate at each stage } \\
\text { Larva survival rate also depends on availability of habitable water and carrying capacity of larva, where habitable water is } \\
\text { dependent on precipitation pattern and proportion of open containers } \\
\text { Fifty percent of the emerging adults are emerged as female adults,contributing to dengue transmission and become } \\
\text { fertilized adult with a delay } \\
\text { Based on temperature dependent adult survival rate and ovarian development rate,fertilized adults are transformed into } \\
\text { gravid adults to lay eggs }\end{array}$ \\
\hline Dengue Transmission Model & $\begin{array}{l}\text { These available adults are transformed into infectious adults based on temperature dependent adult biting rate,vector } \\
\text { infection probability, dew point temperature dependent persistency factor and interaction with infectious host simulated in } \\
\text { host population dynamic model } \\
\text { Infectious adults become effective which is related to temperature dependent delay for EIP and life span. The proportion of } \\
\text { effective infectious adults become available for infection transmission that have longer life span than delay rate } \\
\text { These available effective infectious adults contribute in converting the 'susceptible host' from the 'Host population dynamic } \\
\text { model' to 'Host with infected bloodmeal', based on temperature dependent adult biting rate and host infection probability. } \\
\text { These hosts with infected bloodmeal become infected hosts in the 'Host population dynamic model', with a delay for IIP }\end{array}$ \\
\hline
\end{tabular}

Figure 7: Brief Model Interpretation

The dynamical evolution of the vector population is modeled representing the whole life cycle. The biological events of the life cycle is associated to the transition rates between different stages of life and their dependence and relationship with temperature and water availability has been taken from experimental observations. Egg, larvae and pupae development and survival rate were determined from previous studies, which are associated with temperature and require the availability of water. In the developed model, it has been assumed that there is minimum availability of water to lay eggs. We have considered the development of larvae in density dependent manner, as discussed in few previous literatures (Morin et al, 2013). Larva survival rate is dependent on larva density and carrying capacity of habitable water. Generally, precipitation or artificial storage can make availability of aquatic breeding sites or habitable water. We followed the approach that adequate habitable water has been developed through precipitation, as the study area has continuous pattern of rainfall through the year. Thus, it has been assumed that there is absence of Human-made or artificial water storage due to 
unavailability of field data on artificial storage and as dry condition does not prevail in the study area. On the other hand, larva-sustaining capacity of habitable water is declined during continuous high rainfall scenario due to overflowing effect. So, it has been assumed that continuous pattern of extremely high rainfall reduce the larva sustaining capacity of habitable water and thus habitable water is not considered as proper breeding site under this scenario. Through this development and survival rate, larvas are emerged as pupae and pupaes are converted to emerging adults with temperature dependent development and survival rate. Only 50 percent of the adults are emerged as female adults and thus fertilized as gravid adults after a delay of three days. The transformation from fertilized adults to gravid adults is associated with temperature dependent ovarian development and adult survival rate. In the model, availability of gravid adults has been considered from the initial stage as the study area is vector dominant and always prevail the condition for their development.

The simulated gravid adults from this life cycle model were then transformed into the effective infectious adult. This part of the model represented the interactive infection transmission process between vector and host. The conversion or transformation of gravid vector adults to the effective infectious adults is significantly dependent on the vector-infected host interaction as well as the temperature dependent extrinsic incubation period (EIP), and eventually this transformation dynamic effectively govern the whole process of dengue transmission. The transformation is also related to temperature dependent adult biting rate and vector infection probability. Vector-infected host interaction or adult biting rate can also rely on socioeconomic factors that have not been considered in this study. Sufficient vector-host interaction and vulnerable socioeconomic condition has been considered in the study area. In the developed model, we have also integrated a persistency factor that is related to the effect of dew point temperature on dengue transmission, which has impact on the conversion of gravid adults to infectious adults. This variable is assimilated based on the hypothesis that comparatively higher dew point temperature supports and expedite the propagation of infection. The infectious adults are transformed in to effective infectious adults after the delay for extrinsic incubation period (EIP), depending on temperature effect on EIP. 
The host population dynamic model is compiled with healthy, infected and recovered population. Fifty to ninety percent of the population of the study area has been considered as susceptible host. The percentage has been used as a calibration parameter. These susceptible hosts become 'Hosts with infected bloodmeal' through the interaction with available effective infectious adult vectors, based on temperature dependent adult biting rate and host infection probability. After the delay for intrinsic incubation period (IIP), these hosts are altered to become infected. The infected hosts interact with the gravid adults of the vector population dynamic model, increasing the susceptibility of intensive transformation of gravid adults to infectious adults. The whole cycle of this infection transmission is started with few initial infected host. The number of initial infected host has been collected from real dengue data. The proportion of infected hosts are recovered with a certain recovery rate and again join the susceptible host. The whole modelling framework and background is briefly presented in figures of the appendix.

\section{Data}

We modelled dengue outbreak based on data collected in Iquitos, Peru. The latitude and longitude of the station data is -3.783 and -73.3 respectively. The dengue outbreak data used for Iquitos dates between 2000-2009. Dengue outbreaks were recorded and separated into the four serotypes (DENV1 - DENV4) for each week of these years from U.S. Naval Medical Research Unit 6 (NAMRU-6). For climatic continuous daily observations, we used NOAA's NCEP climate Forecast System (CFS) reanalysis data. CFS reanalysis is a global, high-resolution system to provide the best continuous daily estimate over the period of record. Our collected climate data set include daily precipitation. dew point temperature and air temperature. Figure 8 shows the actual weekly dengue cases of Iquitos for different years. The data for year of 2002, 2004 and 2008 have been used for model calibration and validation as they represent the epidemic environment. 

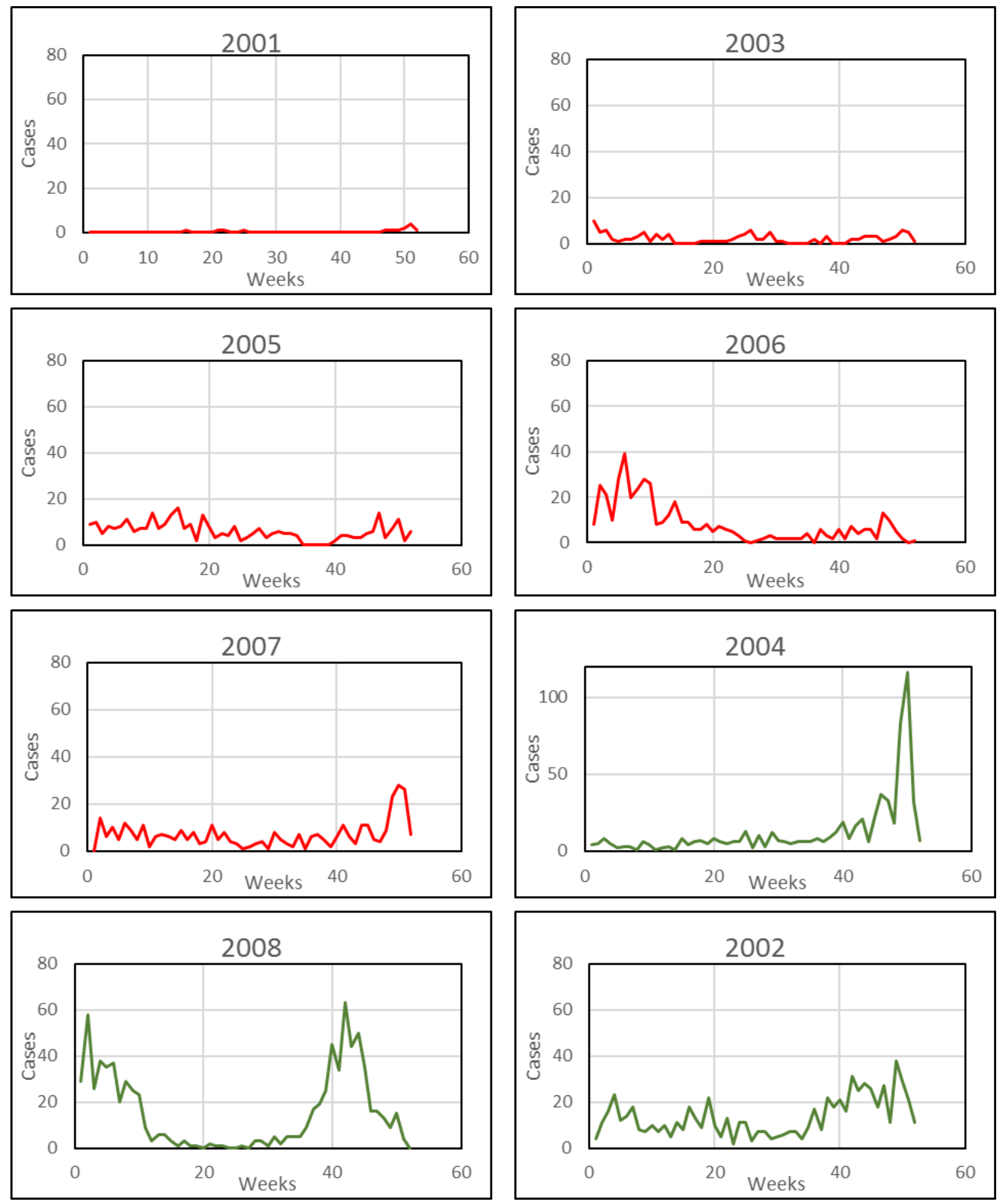

Figure 8: Dengue weekly actual data 


\section{Model Optimization and Calibration}

The model development and calibration approach has been adopted based on experimental field data or empirical equation. Few equations has been developed following our own hypothesis. The unknown or uncertain parameters has been used to calibrate the model. For example, the area of habitable water has been associated with precipitation and has varied with precipitation pattern. Different amount of habitable water for different scenario has been used for calibration of the model, as we have not used any land use pattern or human dwelling density data. For medium range of continuous precipitation, higher amount of habitable water has been considered; whereas lower amount of habitable water has been considered for very low and extremely high precipitation pattern. The other calibrated parameters are larva carrying capacity of the habitable water and proportion of open containers that are uncertain. The term "proportion of open containers' has been used as an indication of socioeconomic condition or lifestyle pattern and utilized to calibrate the model. The initial availability of vectors is a crucial factor, which provides eggs for the initiation of the cycle. Due to unavailability of any trapping or field data, the initial quantification of gravid adults has been considered to be a calibration parameter. In dengue endemic regions, sufficient vector availability is maintained throughout the year and thus general availability is considered from initial stage of the model. These four calibration parameters were used in vector population dynamic part of the model. Delay for intrinsic incubation period (IIP) and recovery rate has been used for calibrating the other part of the model. Literatures suggested that intrinsic incubation period vary between 4 to 8 days (Side \& Noorani, 2013), while recovery rate can also vary within a range depending on the immunity. Literatures suggested the duration of infectiousness of the host to be strongly influential factor for seasonal pattern of dengue transmission and the timing of peak prevalence is dependent on it (Bartley, et al., 2002).Thus recovery rate has been used as a calibration parameter. Moreover, in the study area a proportion of the total population can be immune from the infection, which is an uncertain parameter. The percent of immune people can vary and has been used as a calibration parameter. Monte Carlo approach based vensim sensitivity analysis has been conducted to evaluate the sensitivity of the calibration parameters. Monte Carlo Marcov Chain approach of Vensim has been 
used to identify the values of calibration parameters that resulted in the best fit to the real dengue cases. Table 1 represents the set of calibration parameters with their range. Among these calibration parameters, only two parameters (initial vector availability and initial exposed host) have been changed manually as they were not used as constant in the model and Vensim optimization is modified for constant parameters only. Figure 9 and 10 show the sensitivity of these parameters on the major output parameter of the model (infectious host).

Table 1: Set of calibration parameters

\begin{tabular}{|l|l|}
\hline \multicolumn{1}{|c|}{ Variables } & \multicolumn{1}{|c|}{ Range } \\
\hline Habitable water & $1 * 10^{\wedge} 4$ to $8^{*} 10^{\wedge} 6$ \\
\hline Carrying capacity & $0.5-1$ \\
\hline Proportion of open container & $0.1-1$ \\
\hline Initial vector availability & $1 * 10^{\wedge} 4$ to $1 * 10^{\wedge} 8$ \\
\hline Intrinsic incubation period (IIP) & $4-8$ days \\
\hline Recovery rate & $0.05-0.1$ \\
\hline Initial exposed host & 50 to 90 percent of the total population \\
\hline
\end{tabular}

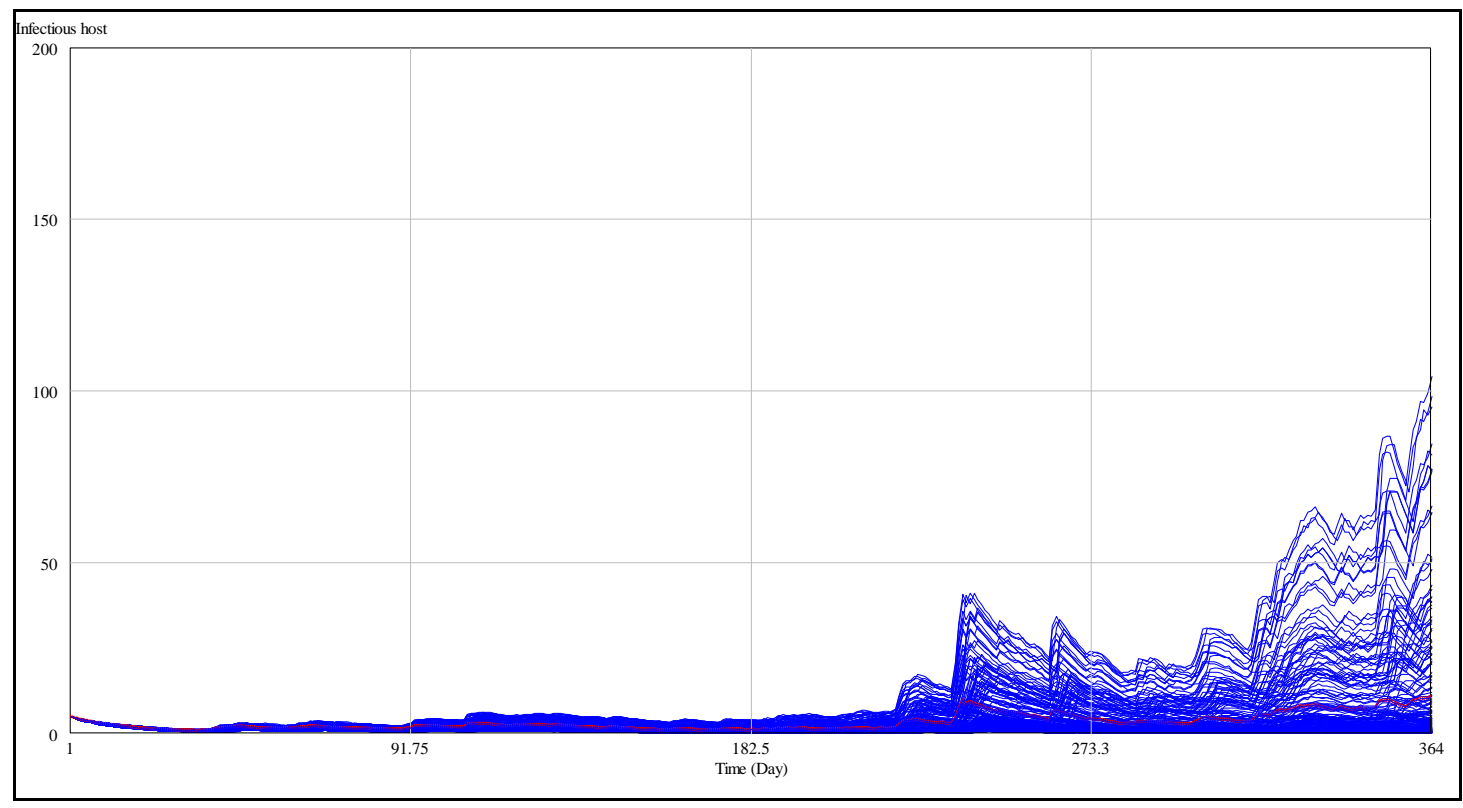

Figure 9: Sensitivity graph for all parameters 


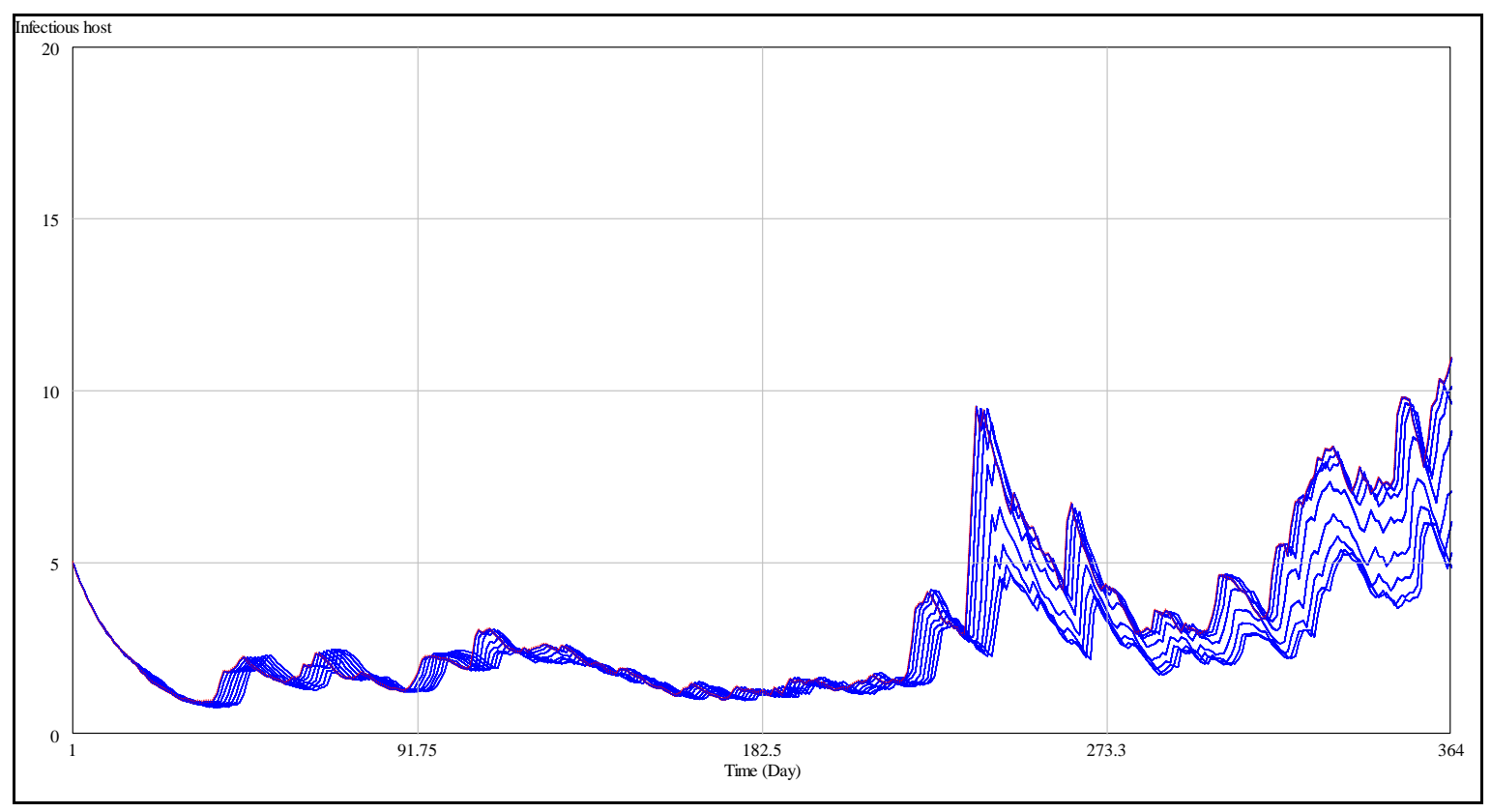

Figure 10: Sensitivity graph without recovery rate

Calibration of model requires simulating thousands of combinations of different parameter values. Vensim is a visual modeling tool that allows simulation, optimization and sensitivity analysis for an established system dynamic model (Lin, et al., 2012). Literatures suggested Vensim as an effective tool to connect the simulation with optimization and to choose sensitive parameters to get optimum output (Kasperska, et al., 2014). In system dynamic models, there are many parameters and the effect of their variation on model simulation is significant. Vensim follows Markov Chain Monte Carlo approach to adjust the parameters to get the optimal match between model behavior and the real data. In this approach, the output is optimized under uncertain environment using different combinations of calibration parameters within a certain range (Lin, et al., 2012). Monte Carlo Multivariate sensitivity analysis of Vensim can be used as an entrance for optimization as it facilitates in selecting the sensitive parameters for optimization (Kasperska, et al., 2013). Different literatures used optimization set up of Vensim where certain range and weight can be identified for each payoff or calibration parameter. Literatures suggested Vensim's optimization and sensitivity set ups as efficient approach as it allows searches within confidence bounds or optimal values to provide the best fit to the 
real data. The process of optimization is advantageous as it allows providing weights to different calibration parameters according to its sensitivity (Kasperska, et al., 2013). The equations and relationships used for the model are provided in figure 16 to 18 in appendix. All the equations or relationships used are for daily scenario.

\section{Results and Simulations}

The model has been calibrated for 2002 dengue epidemic and validated for 2004 and 2008 epidemics, comparing the weekly simulated cases with the weekly actual cases. The model simulation has provided daily cases and weekly cases were determined through summation of daily cases. All the figures of model simulations are provided in appendix. Figure 11 depicts the simulation of the calibrated model, which has been used for validation of the model for 2004 and 2008. Figure 12 shows the weekly actual cases and model cases for the year 2008. It has been observed that there was an initial peak in dengue cases, which diminished after tenth week and the next significant rise started after thirty fifth week following almost six months of moderate scenario. The simulation has been

observed to be competent in capturing the declining trend during tenth week and increasing trend during thirty fifth week. The model is capable of replicating the moderate scenario in the mid-year and peak scenario during the starting and end of the year. But, the model simulated the year end peak with a lag compared to the actual scenario. Figure 13 shows the comparison between simulated and actual cases for 2004. During 2004, the peak scenario has been observed at the end of the year after continuous moderate condition for the first nine months. The cases started rising after the fortieth week, which has been captured by the model. The model has been also capable of simulating the peak week, but with comparatively lower number of cases than the actual scenario.

The initial peak during 2008 was resulted from previous year scenario as the simulation was started with large number of initially infected cases. The cases started decreasing due to continuous comparative lower temperature and dew point temperature, which was not persistent enough for efficient infection transmission. The precipitation scenario has not been observed to have much impact on the case pattern as vector availability was maintained throughout the year. The mid year condition influenced the end 
peak as efficient transmission was allowed by temperature and dew point temperature scenario. During 2004, the scenario started with lower number of initially infected host. The end of the year peak condition was generated from mid-year persistent higher temperature and dew point temperature, the effect of which resulted with a lag at the end of the year.

Figure 14 and 15 show the probability of exceedance plot for actual and modeled cases for 2008 and 2004 respectively. The probability of exceedance has been computed for actual and modeled cases and plotted with normal distribution in order to evaluate the model competent. The graphs depict that the model is significantly competent and efficient to replicate the dengue epidemic environment as the exceedance plots of actual and simulated cases follow almost similar distribution.

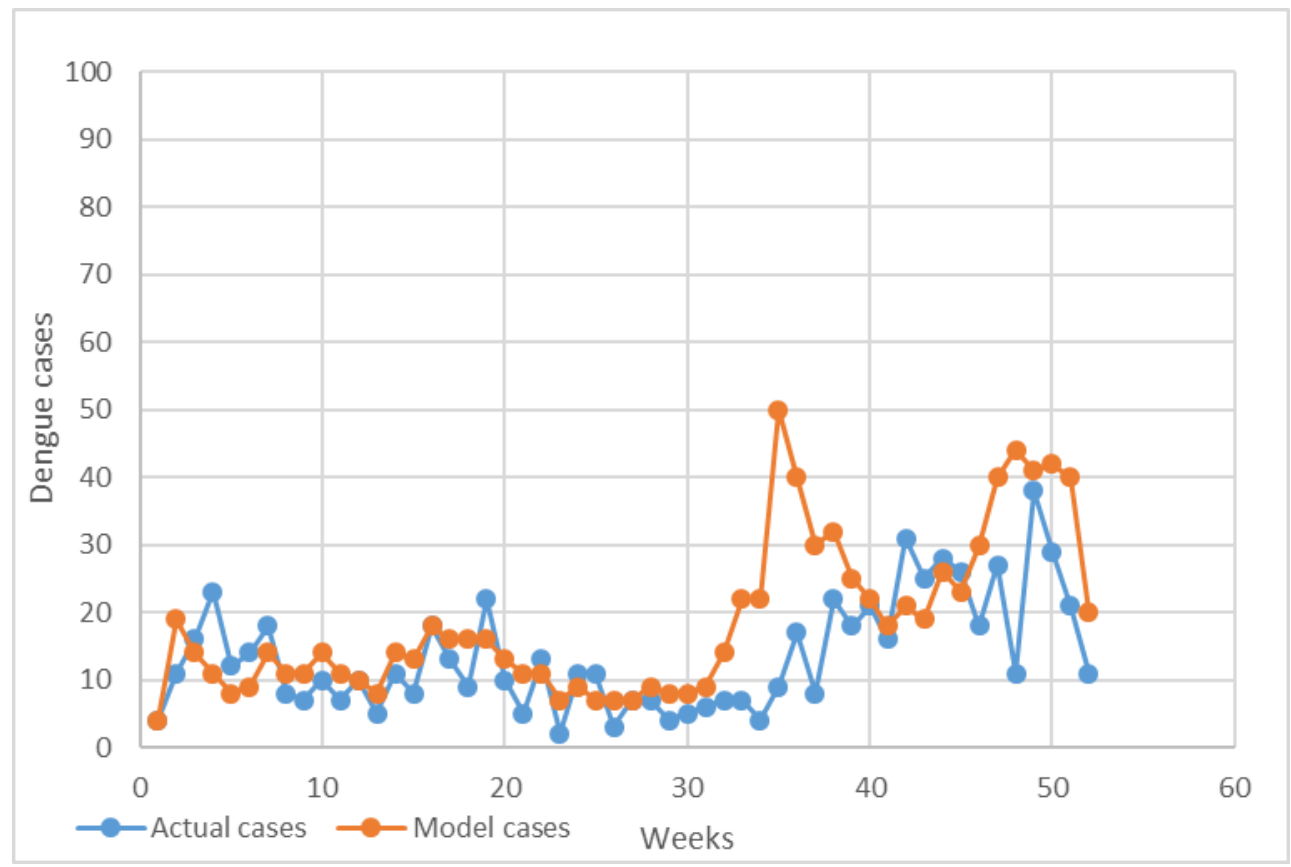

Figure 11: Model simulated and actual cases for 2002 (Model Calibration) 


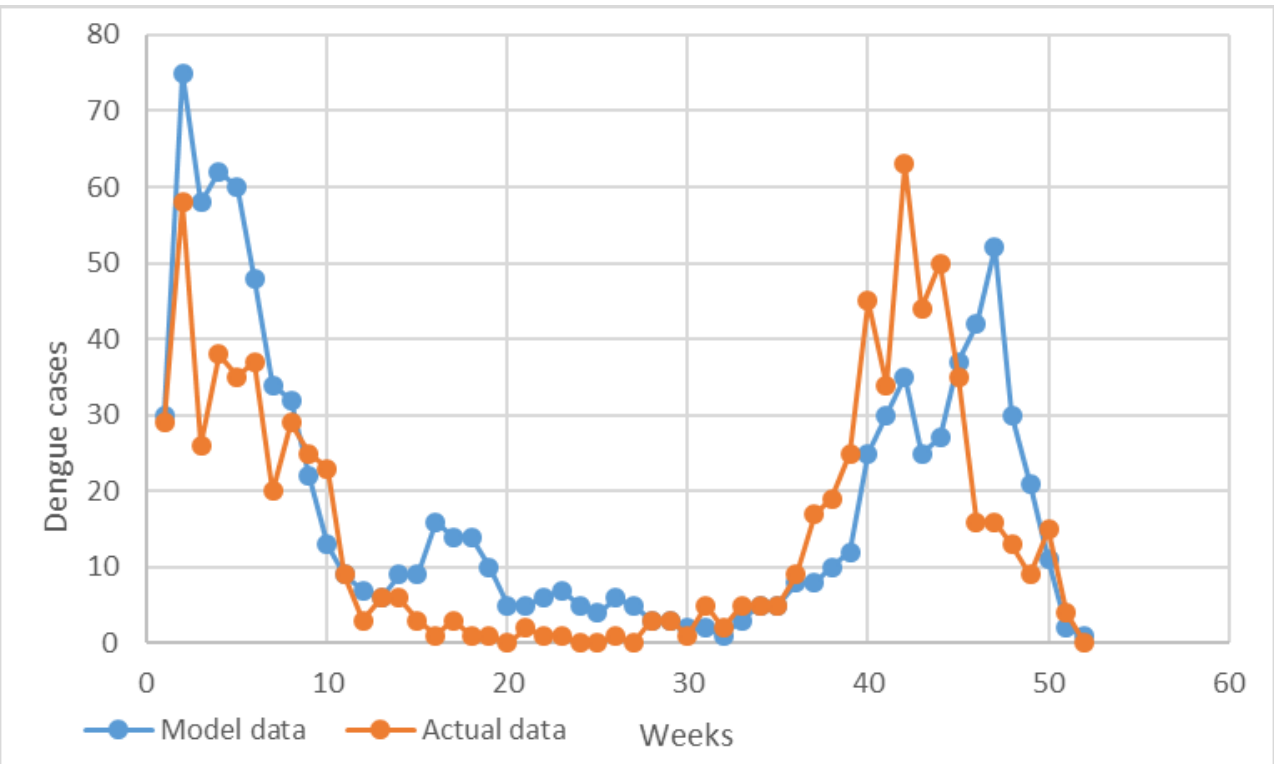

Figure 12: Model simulated and actual cases for 2008 (Validation)

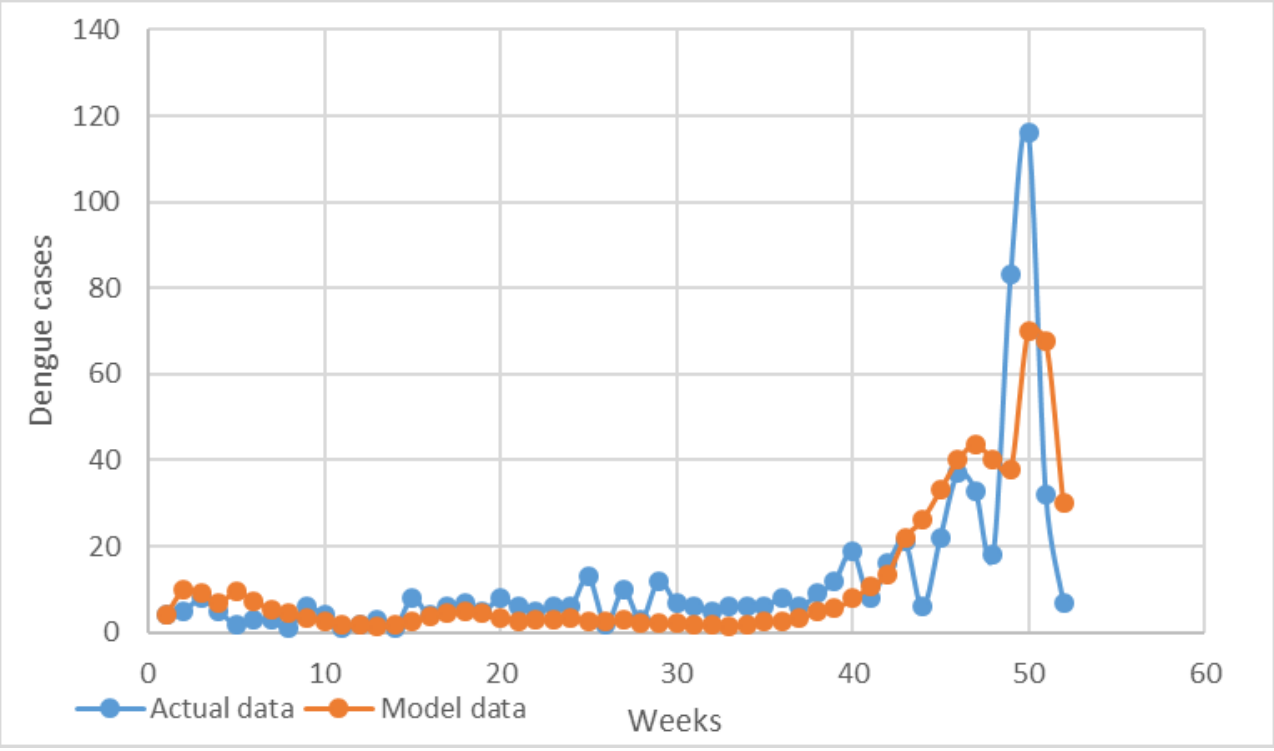

Figure 13: Model simulated and actual cases for 2004 (Validation) 


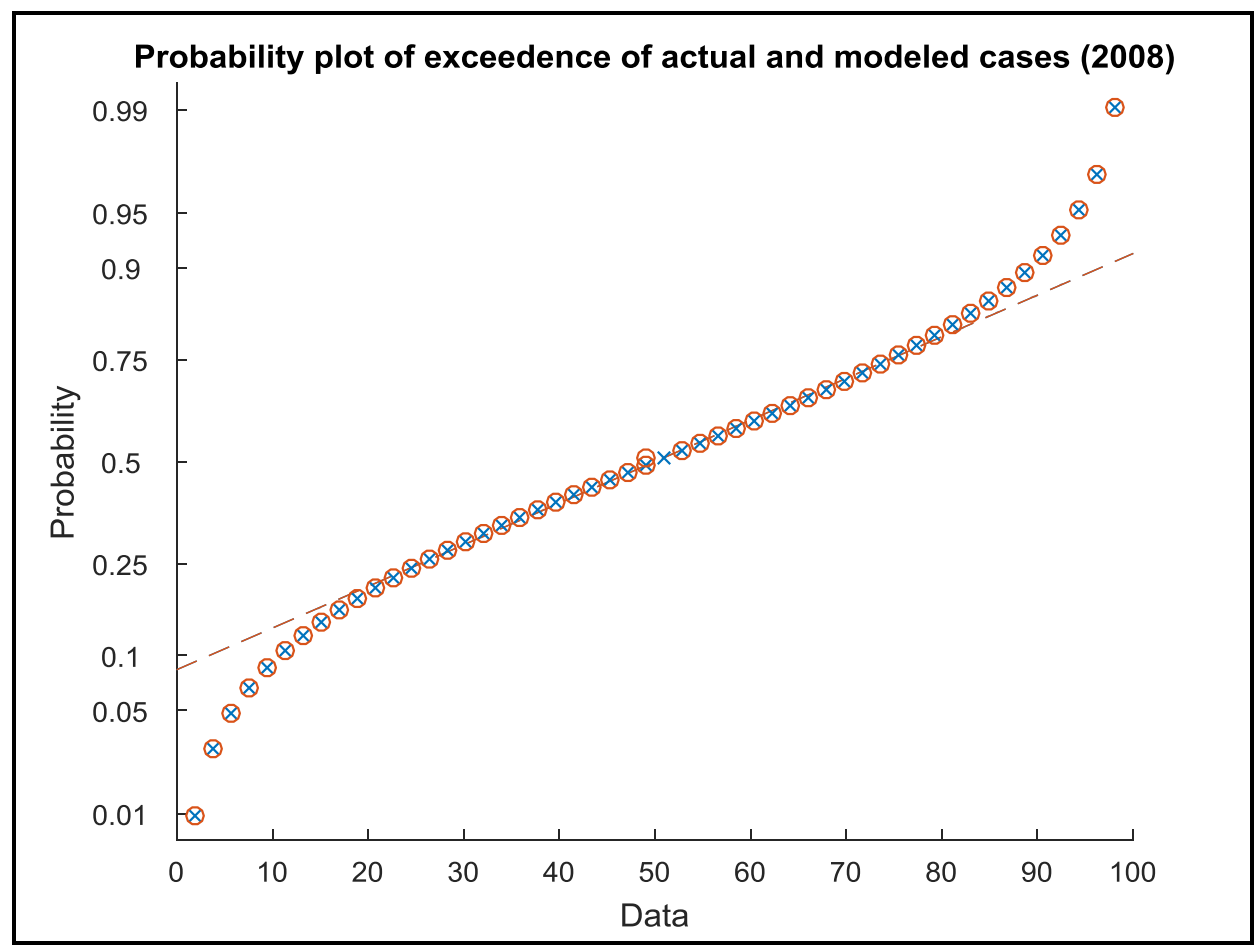

Figure 14: Probability of exceedance of actual and medeled cases (2008)

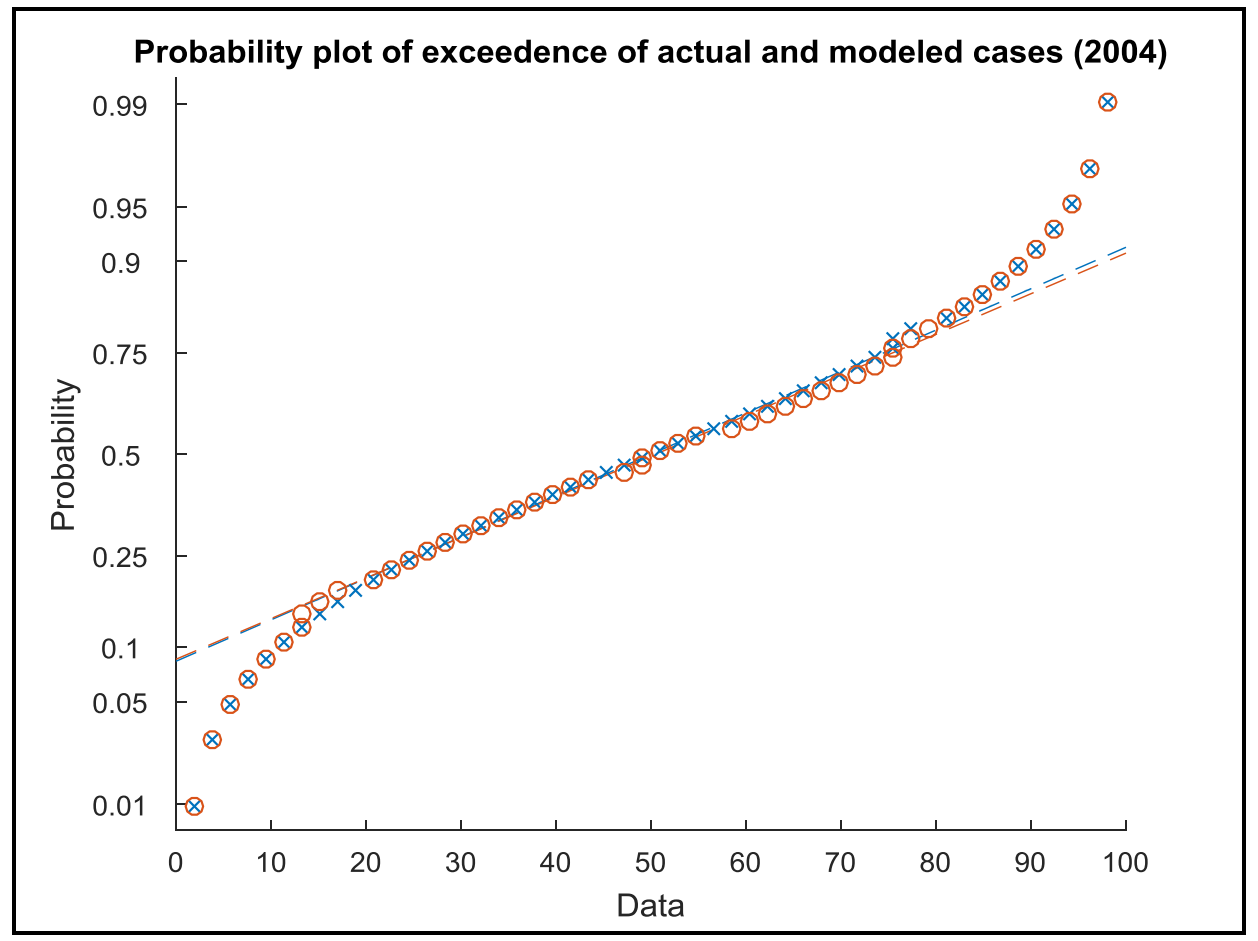

Figure 15: Probability of exceedance of actual and modeled cases (2004) 


\section{Conclusion}

\subsection{Summary}

The model highlighted the overall physical system of the transmission process using the linkage and interaction between different parameters to explain the physical phenomena contributing in timing and scale of dengue epidemic. The sole objective was to develop a simple model to represent the actual scenario triggering dengue epidemic considering the most important features of vector population dynamics, transmission mechanism and environmental linkages. By simulating 2004 and 2008 outbreaks, the model has defined the conditions generating the peak, which can be utilized in future prediction and planning for control strategies. As the model has replicated the peak condition, it can facilitate in assessing the persistent environmental condition with associated lag that can cause significant proliferation in transmission scenario.

From a practical point of view, this model can facilitate in understanding the triggering environmental condition that can cause local or regional epidemic scenario. As we have used grid based remote sensing data, it is possible to make local risk maps or evaluate local risk patterns using the framework and concept of this model.

From a methodological point of view, few detailing and augmentation can make the model more effective. There is probability of vertical transmission of infection processed by transmission to the descendants by infected female vectors (Side \& Noorani, 2013). However, the rate of this transmission is very low (Side \& Noorani, 2013) and has not been incorporated in the model. The model has been developed considering only one vector species, while aedes albopictus can be another potential vector with different dynamics. Moreover, the model has been developed considering precipitation induced habitable water, whereas artificial or human-made habitable water can also prevail in comparative dry condition or depending on socioeconomic scenario. Thus, availability of different type of habitable water has to be considered for reframing the model for different study area with disparate precipitation pattern. Furthermore, the socioeconomic condition and life style has significant impact on vector-host interaction and thus regulate the 
transmission process, which has not been incorporated in the model. Only one calibration parameter (proportion of open container) has been used, which indirectly indicate lifestyle pattern. Also, survival rate of adult vector has been considered to be effected only by temperature, although vector control activities and socioeconomic pattern has significant influence in this regard.

The model has been validated using actual data to show that it can almost generate the actual scenario capturing the temporal pattern of peak. However, incorporation of parameters regarding local socioeconomic condition may facilitate in adaptation of the model for different locality with more accurate simulation.

\subsection{Future Work}

The model has been simulated for Iquitos; however it has been developed in such a way that it can be adopted for different regions of available vectors with different environmental data and population density. The transformation is dependent on availability of environmental data, demographic data and actual case data. The motivation of future work is to develop the model using this concept and framework for other dengue vulnerable cities. Incorporation of some social parameters will be another purpose and direction of future work.

With appropriate reparameterisation, this model can be modified for other diseases (zika,chikungunya) with same vectors. This model can be modified for these diseases if demarcation among their environmental transmission condition is developed. With development of demarcation among transmission condition of these diseases, we have plan to develop model for zika and chikunguniya using the same concept. 


\section{Appendix}

Vector life cycle

Equations used/Constant

Conditions

Eggs=egg batching rate* ${ }^{*}$ gravid adult

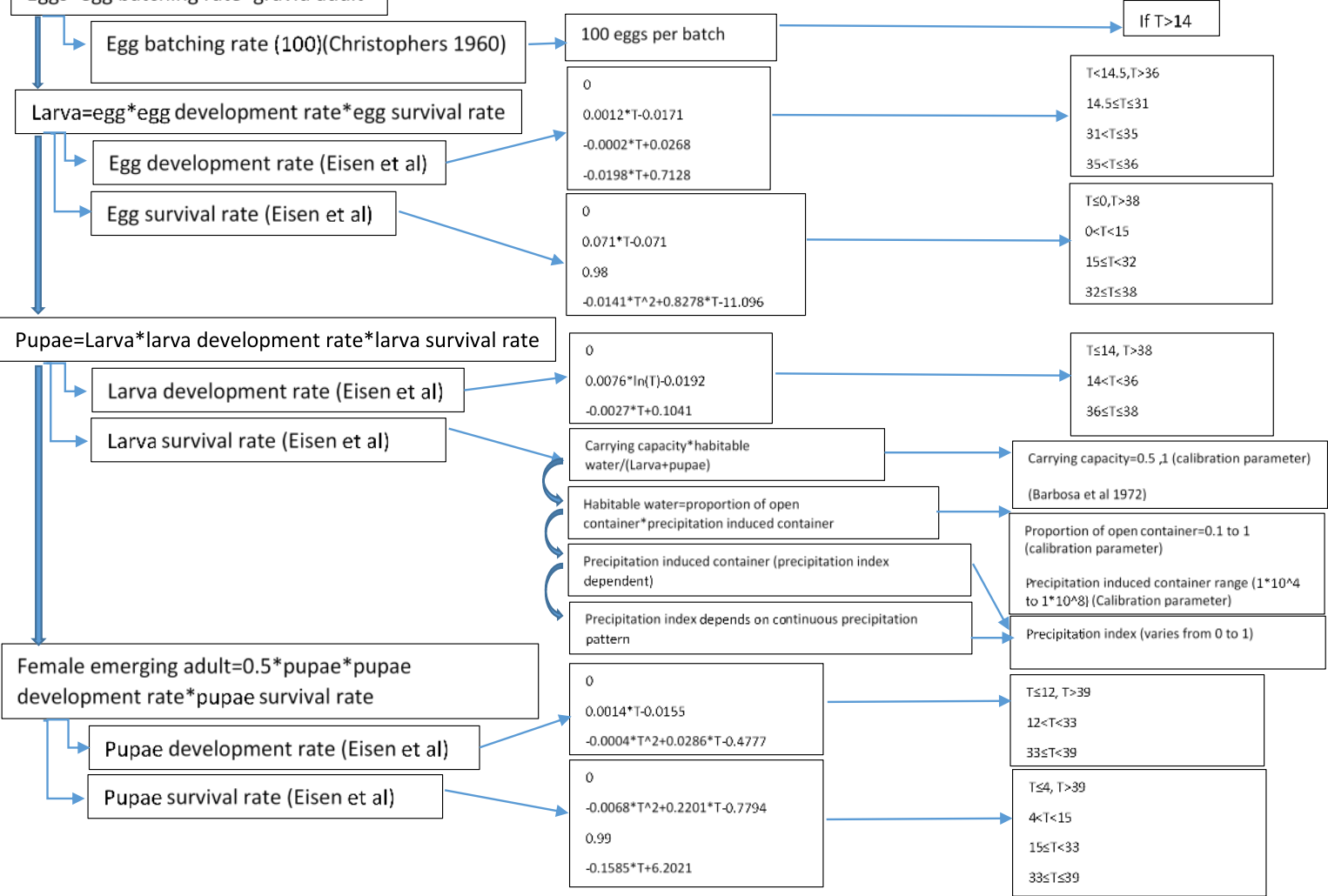

Figure 16: Vector life cycle model interpretation (part

1) 


\section{Vector life cycle}

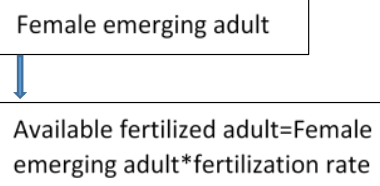

\begin{tabular}{|c|c|c|c|}
\hline \\
\hline & $\rightarrow \quad \begin{array}{c}\text { Fertilization rate (Christophers } \\
1960)\end{array}$ & $\begin{array}{l}0.33,0.5,1 \text { (calibration } \\
\text { parameter) }\end{array}$ & $\mathrm{T}>17$ \\
\hline \multicolumn{4}{|c|}{ Fertilized adult } \\
\hline \multicolumn{4}{|l|}{$\rrbracket$} \\
\hline \multicolumn{2}{|c|}{$\begin{array}{l}\text { Gravid adult=fertilized adult*adult survival } \\
\text { rate*ovaporian development rate }\end{array}$} & \multirow{2}{*}{$\begin{array}{l}0.01 \\
0.88 \\
\end{array}$} & \multirow{2}{*}{$\begin{array}{l}\mathrm{T}<1, \mathrm{~T}>40 \\
1 \leq \mathrm{T} \leq 40\end{array}$} \\
\hline \multirow{3}{*}{$\longrightarrow$} & \multirow{2}{*}{$\begin{array}{l}\text { Adult survival rate (Muir and Kay } \\
\text { 1998,Conway et al 1974) }\end{array}$} & & \\
\hline & & 0 & \multirow{2}{*}{$\mathrm{T} \leq 20$} \\
\hline & $\begin{array}{l}\text { Ovaporian development rate (Christophers } \\
\text { 1960, Focks et al 1993) }\end{array}$ & 0.01 & \\
\hline \multicolumn{2}{|r|}{$\begin{array}{l}\text { Available adult for biting=Gravid adult* gonotrophic } \\
\text { development rate }\end{array}$} & 0/very low & \multirow{2}{*}{$\begin{array}{l}T<20, T>32 \\
20 \leq T \leq 22 \\
22<T \leq 24\end{array}$} \\
\hline \multirow[t]{5}{*}{$\Rightarrow$} & \multirow{5}{*}{$\begin{array}{l}\text { Gonotrophic development rate } \\
\text { (Focks et al 2000) }\end{array}$} & 0.199 & \\
\hline & & 0.240 & $24<T \leq 26$ \\
\hline & & 0.288 & $26<T \leq 28$ \\
\hline & & 0.344 & $28<T \leq 30$ \\
\hline & & 0.411 & $30<T \leq 32$ \\
\hline
\end{tabular}

Figure 17: Vector life cycle model interpretation (part 2) 


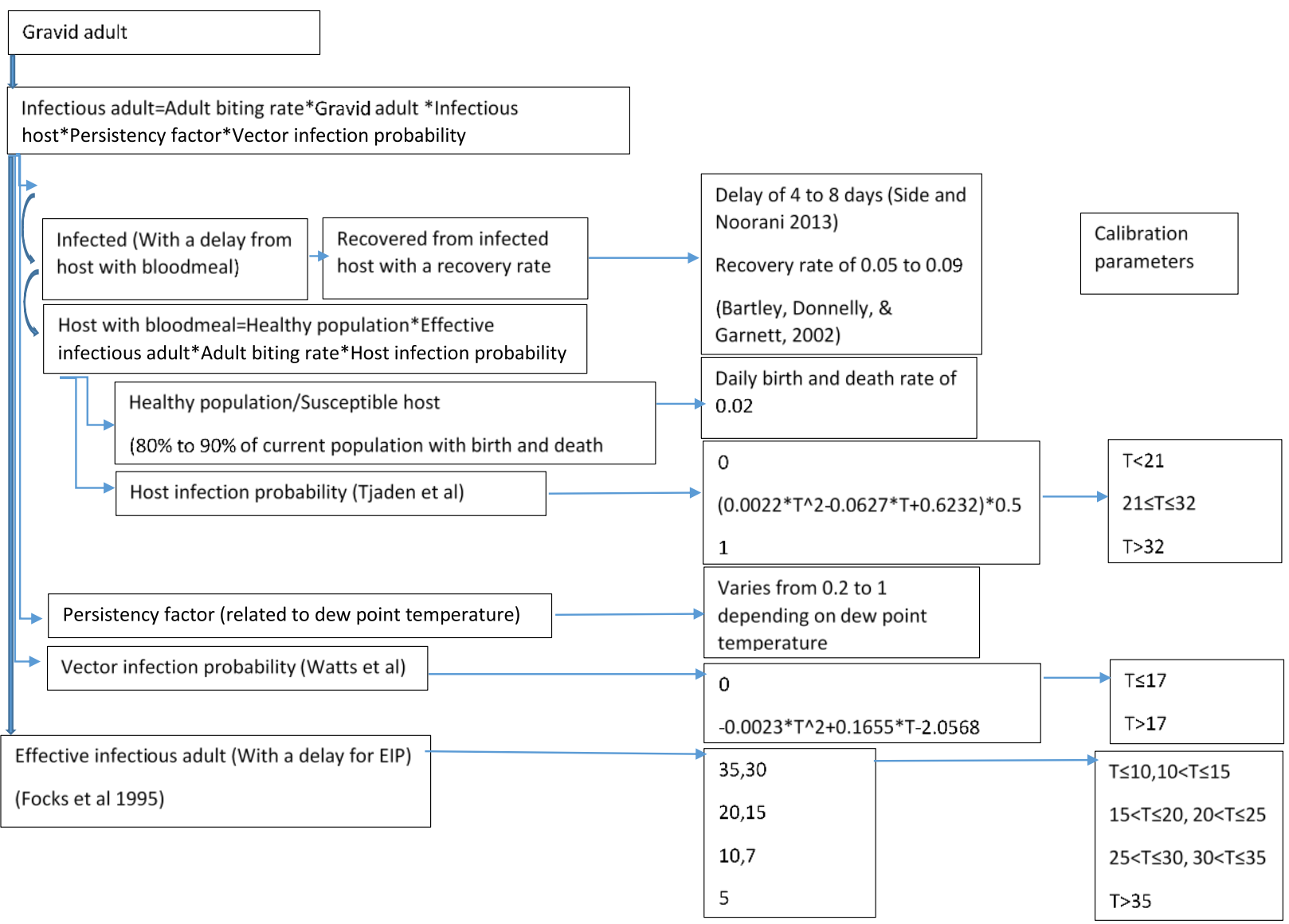

Figure 18: Transmission and population model interpretation 


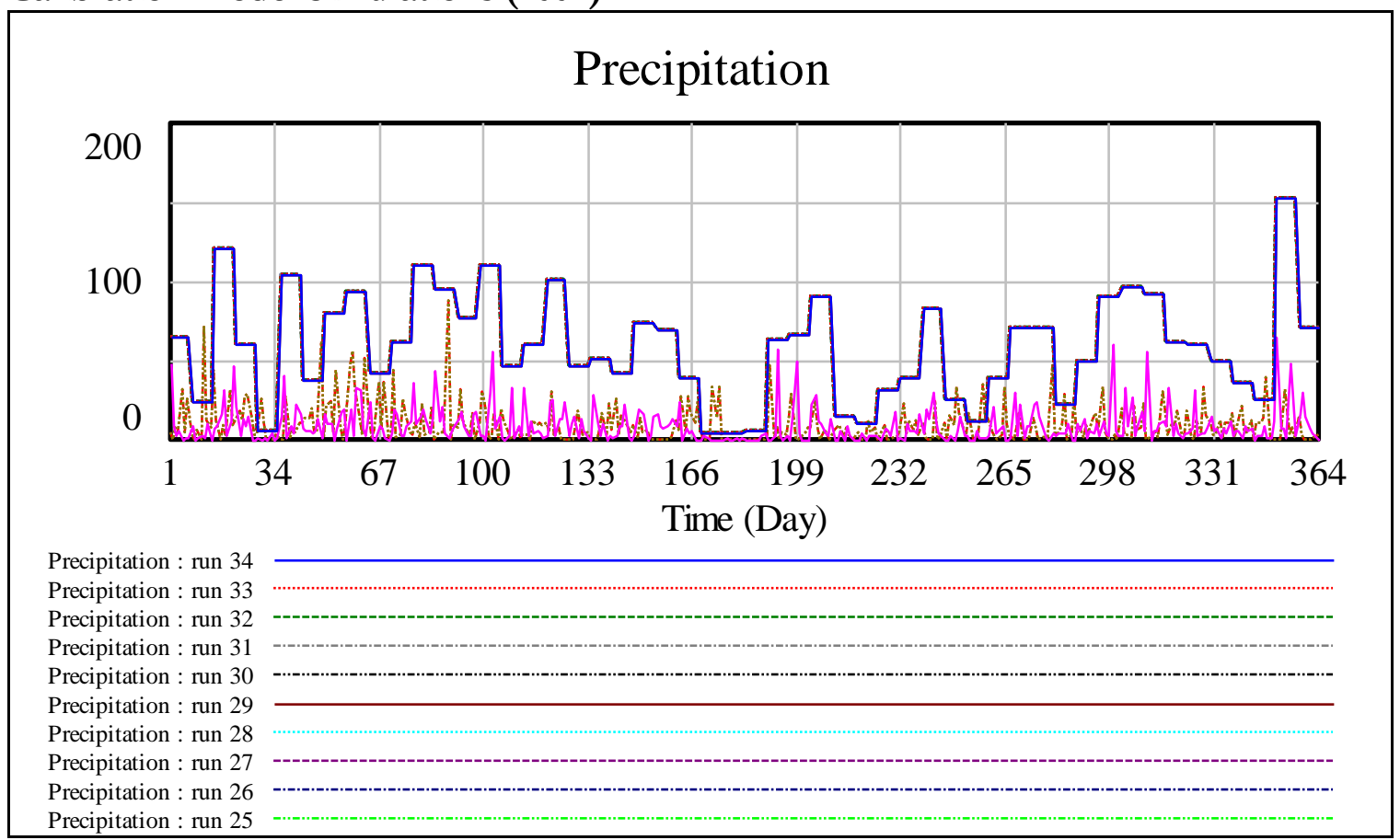

Figure 19: Precipitation Simulation

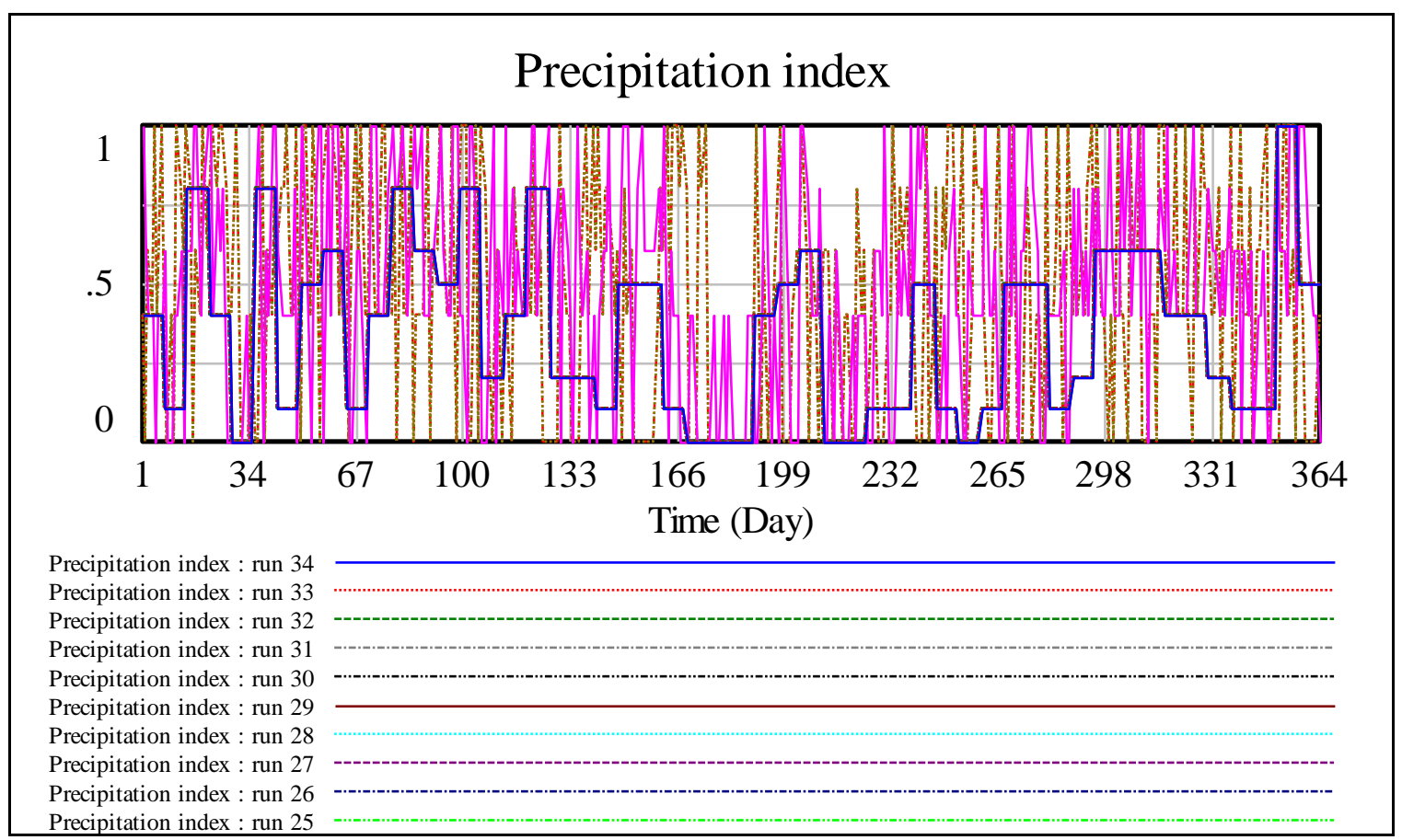

Figure 20: Precipitation index simulation 


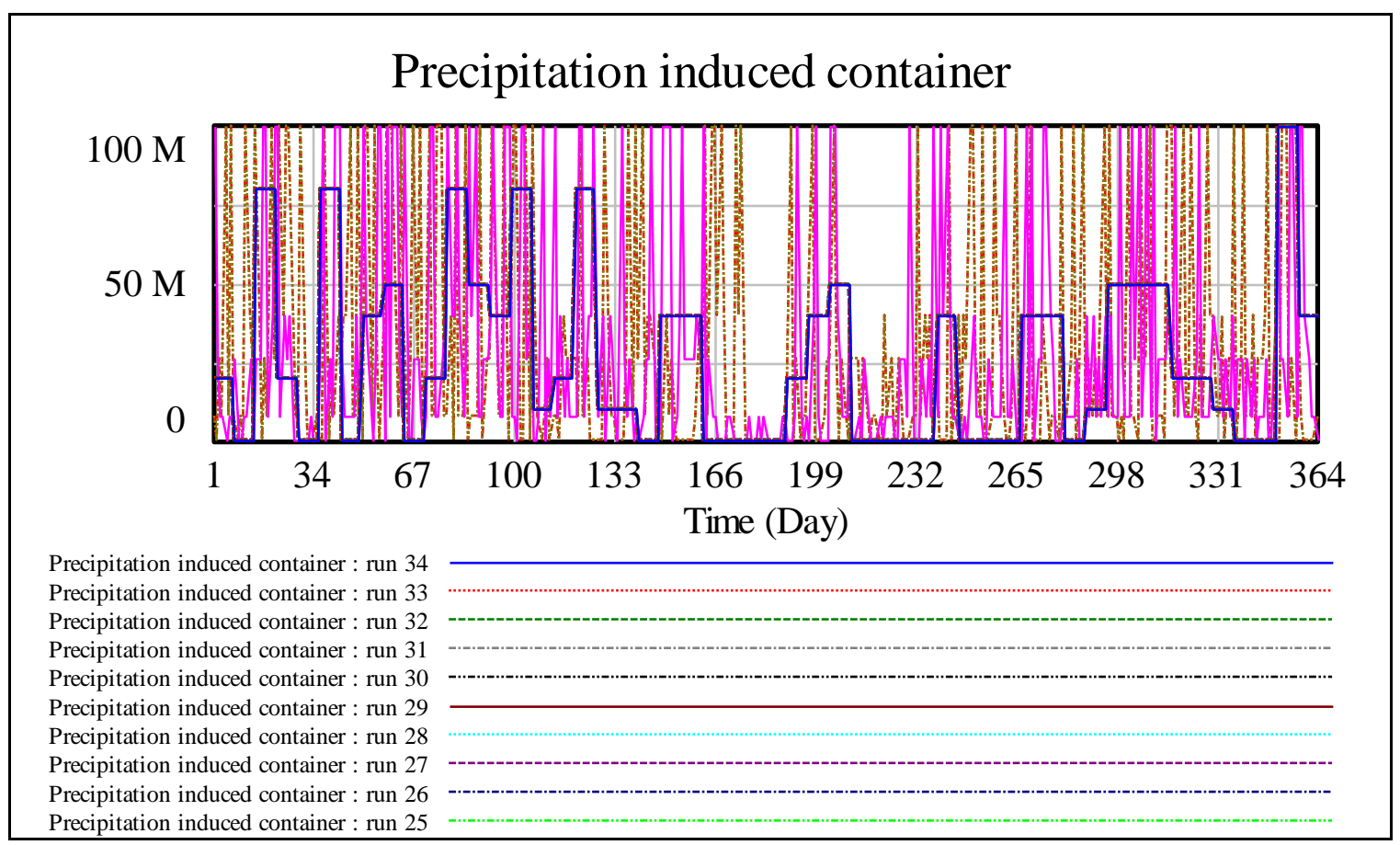

Figure 21: Precipitation induced container simulation

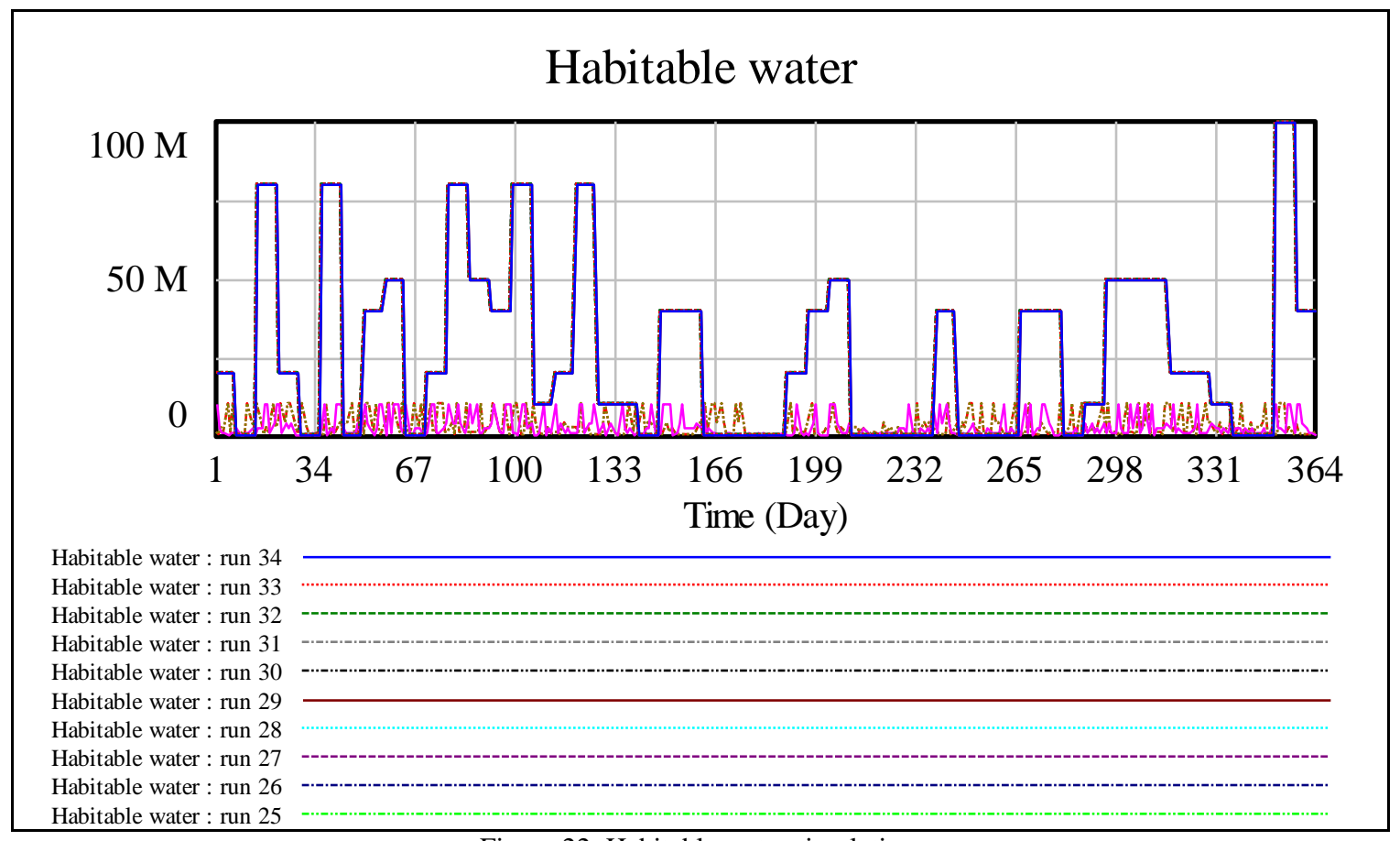

Figure 22: Habitable water simulation 


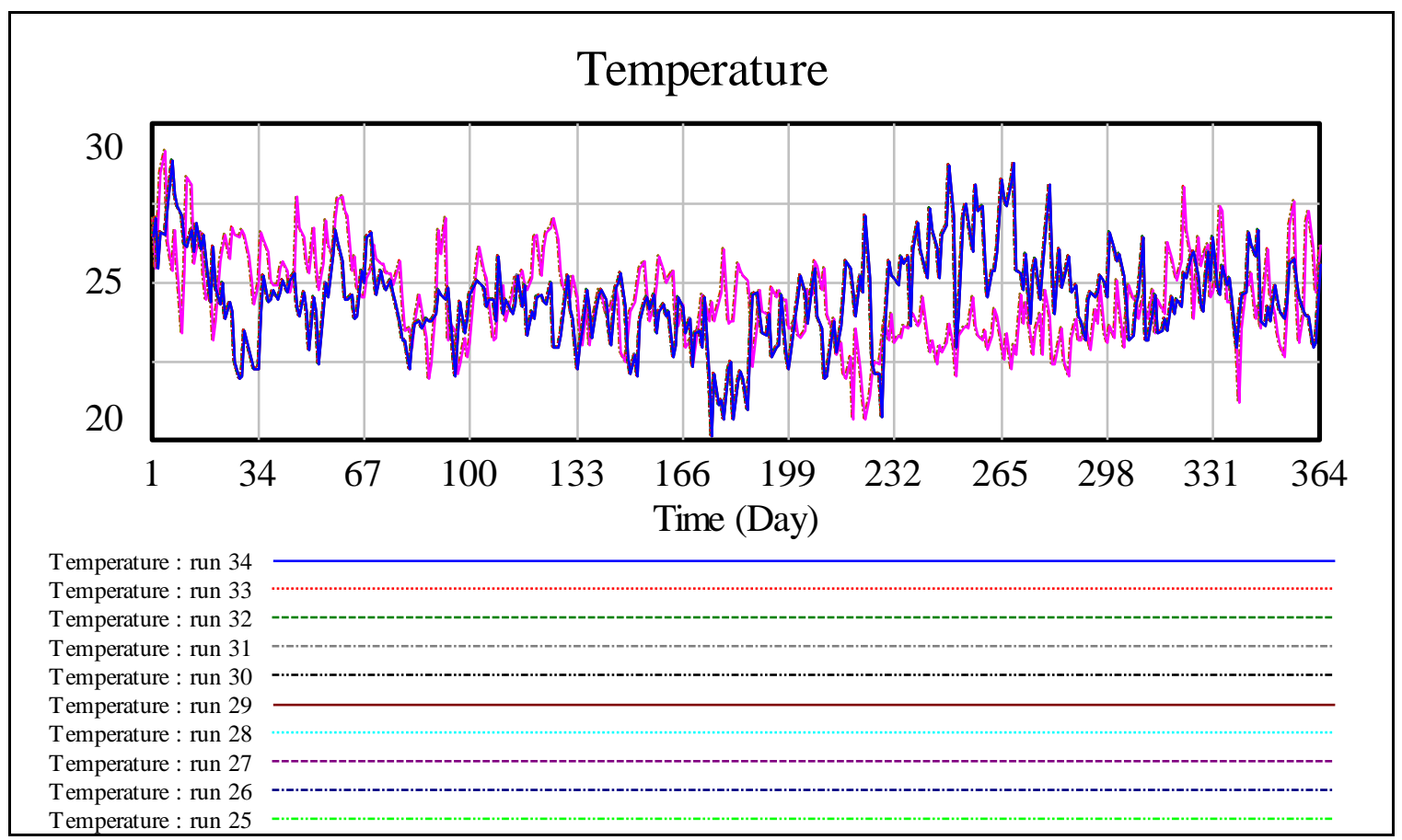

Figure 23: Daily temperature simulation

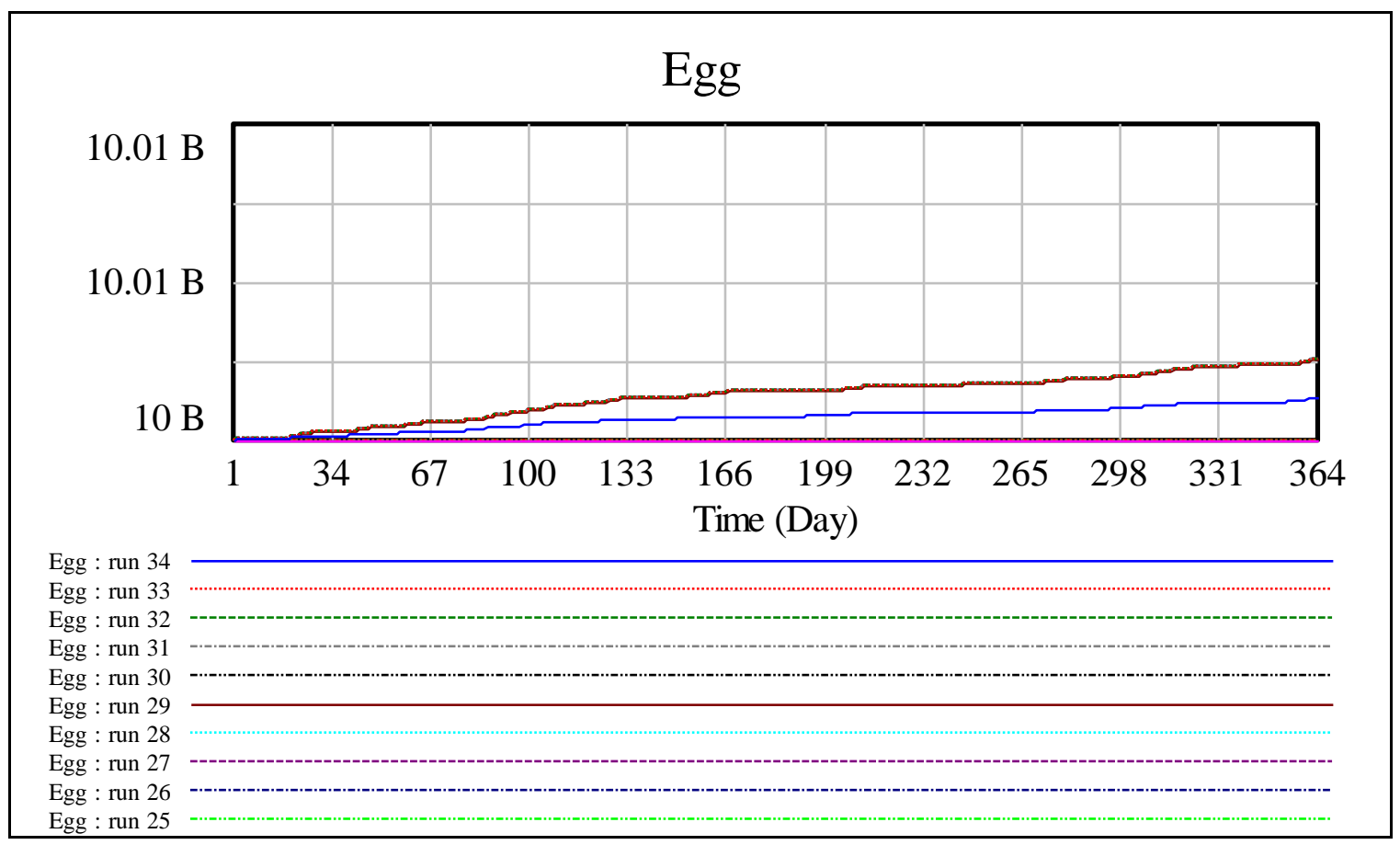

Figure 24: Vector egg simulation 


\section{Egg development rate}

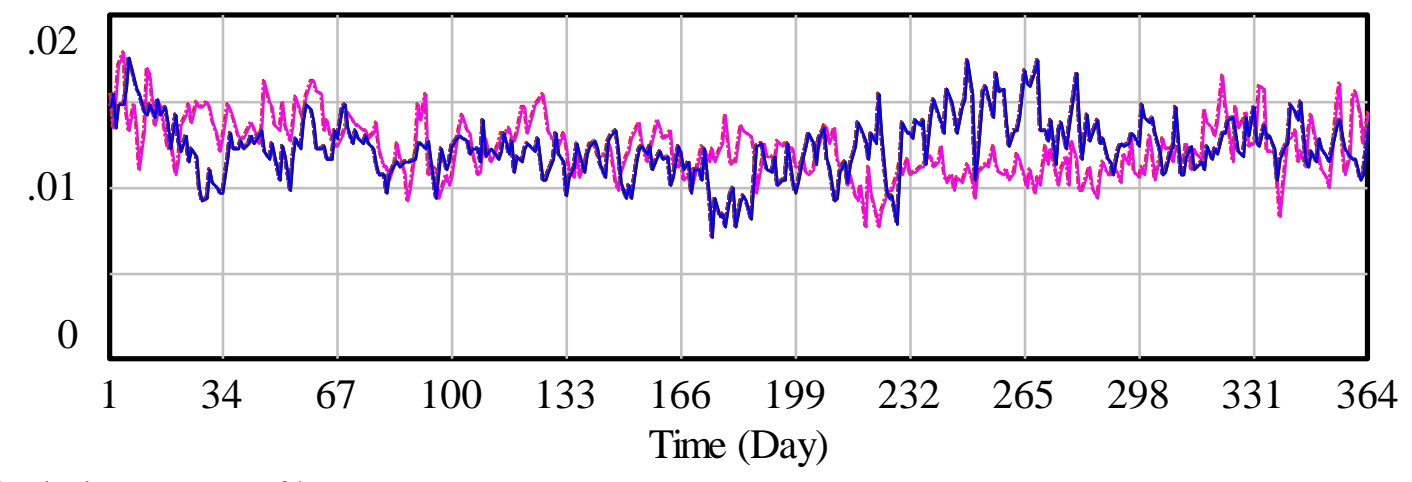

Egg development rate : run 34

Egg development rate : run 33

Egg development rate : run 32

Egg development rate : run 31

Egg development rate : run 30

Egg development rate : run 29

Egg development rate : run 28

Egg development rate : run 27

Egg development rate : run 26

Egg development rate : run 25

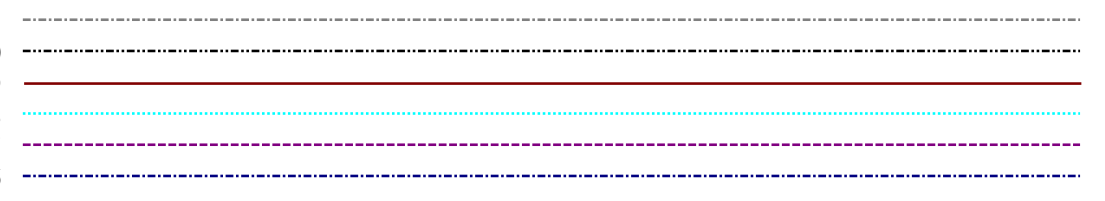

Figure 25: Egg development rate simulation

\section{Egg survival rate}

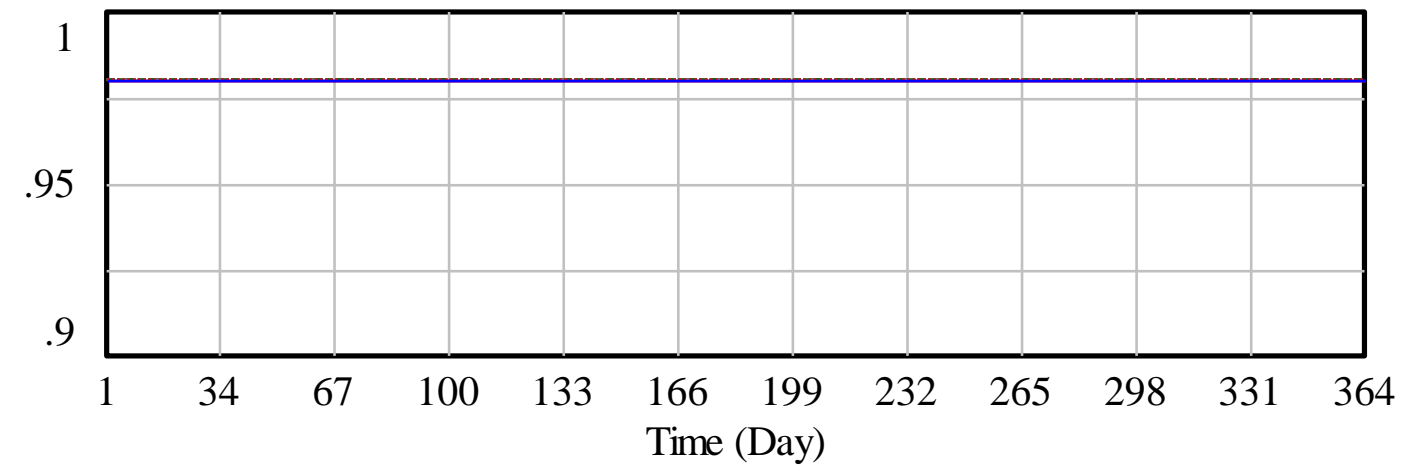

Egg survival rate : run 34

Egg survival rate : run 33

Egg survival rate : run 32

Egg survival rate : run 31

Egg survival rate : run 30

Egg survival rate : run 29

Egg survival rate : run 28

Egg survival rate : run 27

Egg survival rate : run 26

Egg survival rate : run 25

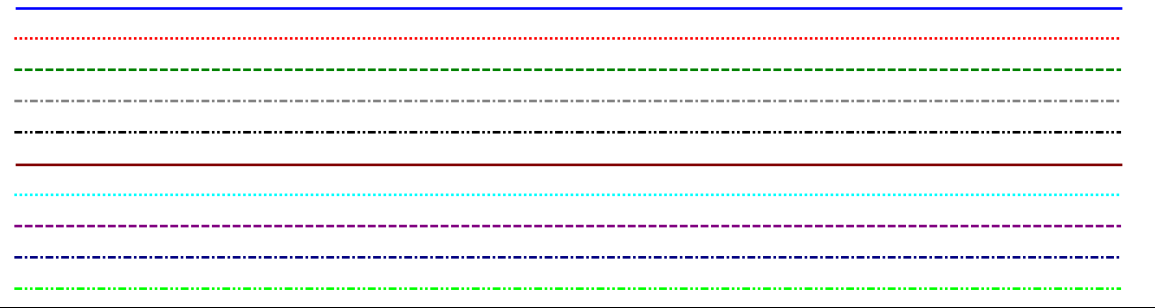

Figure 26: Egg survival rate simulation 


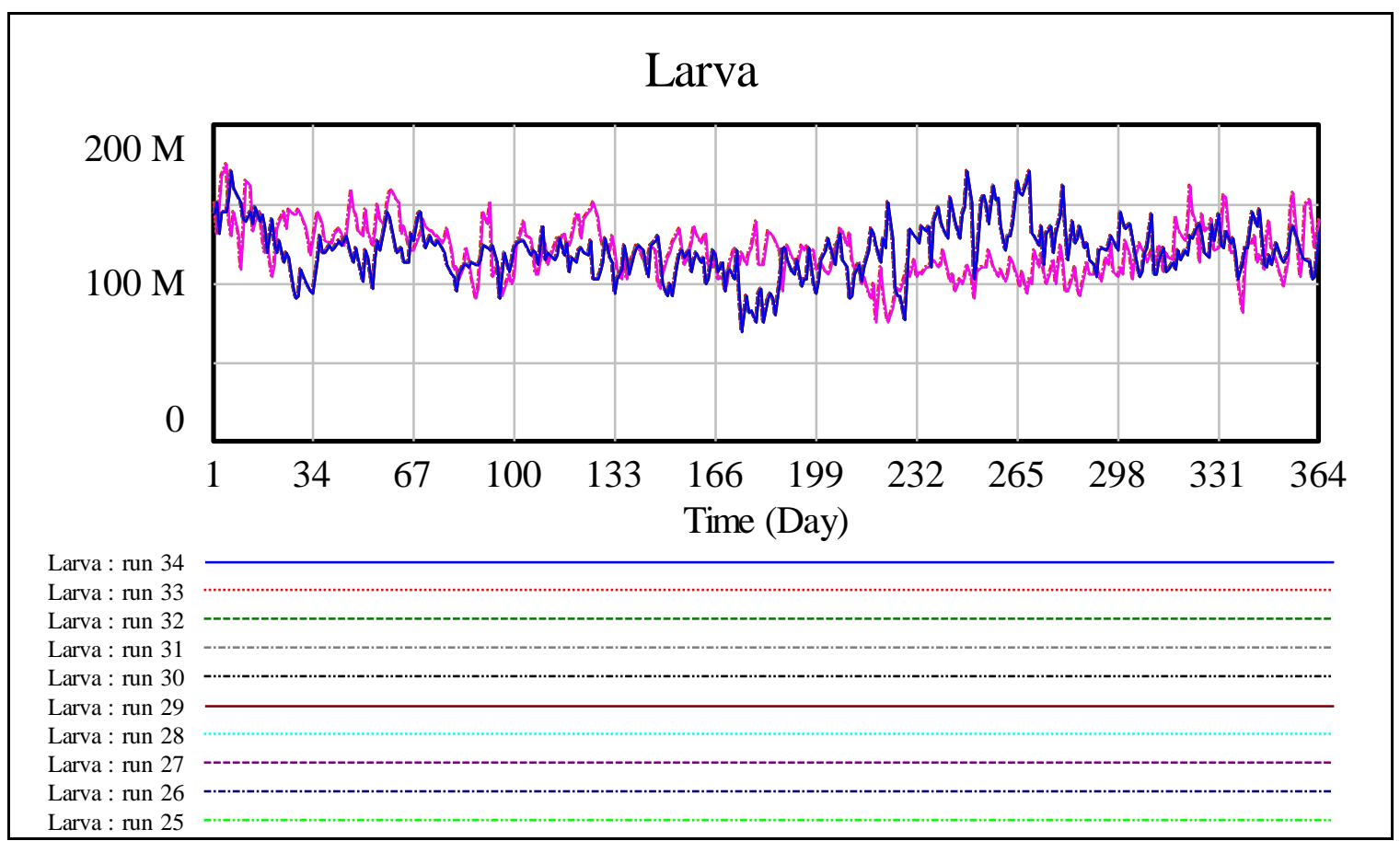

Figure 27: Larva simulation

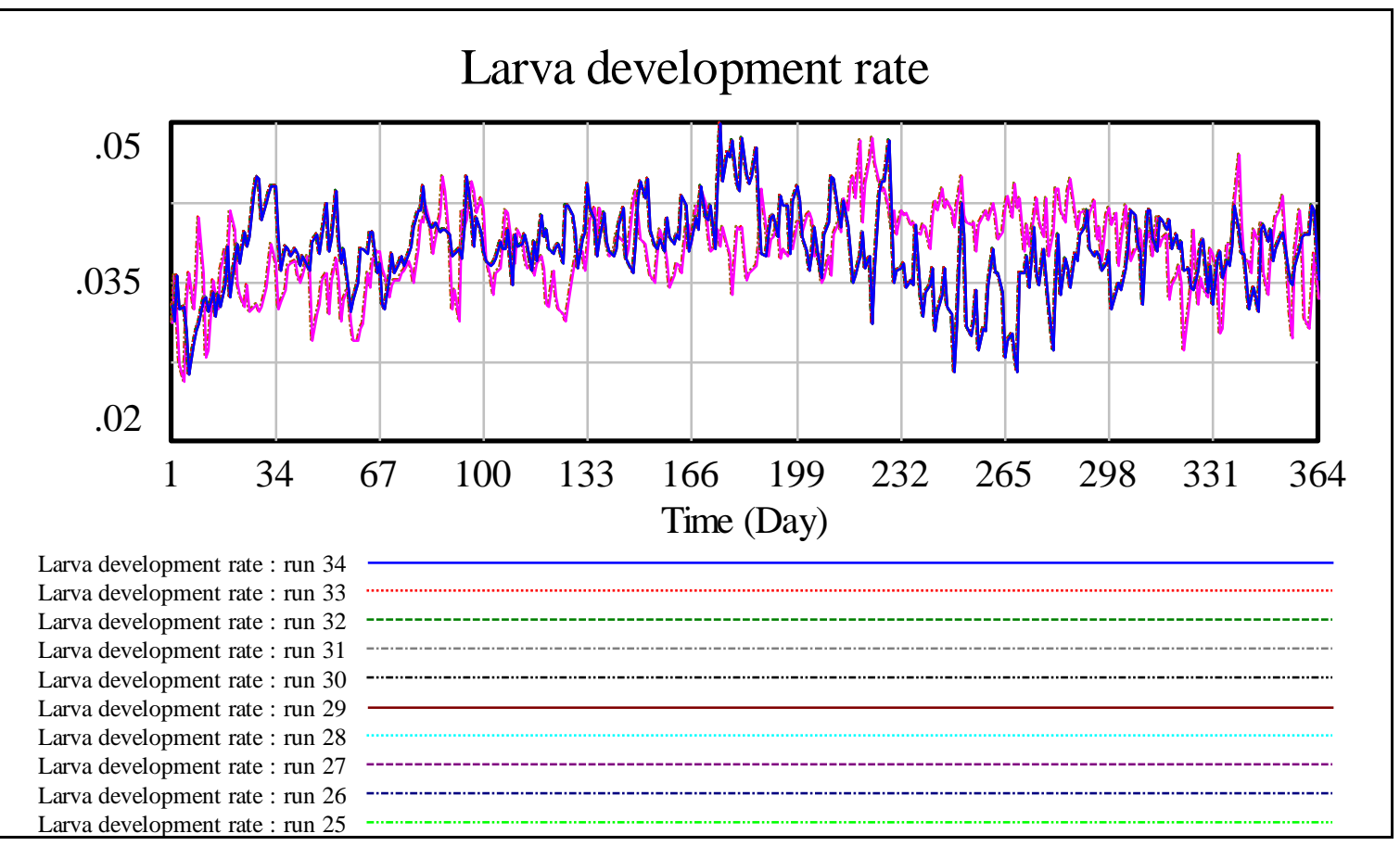

Figure 28: Larva development rate simulation 


\section{Larva survival rate}

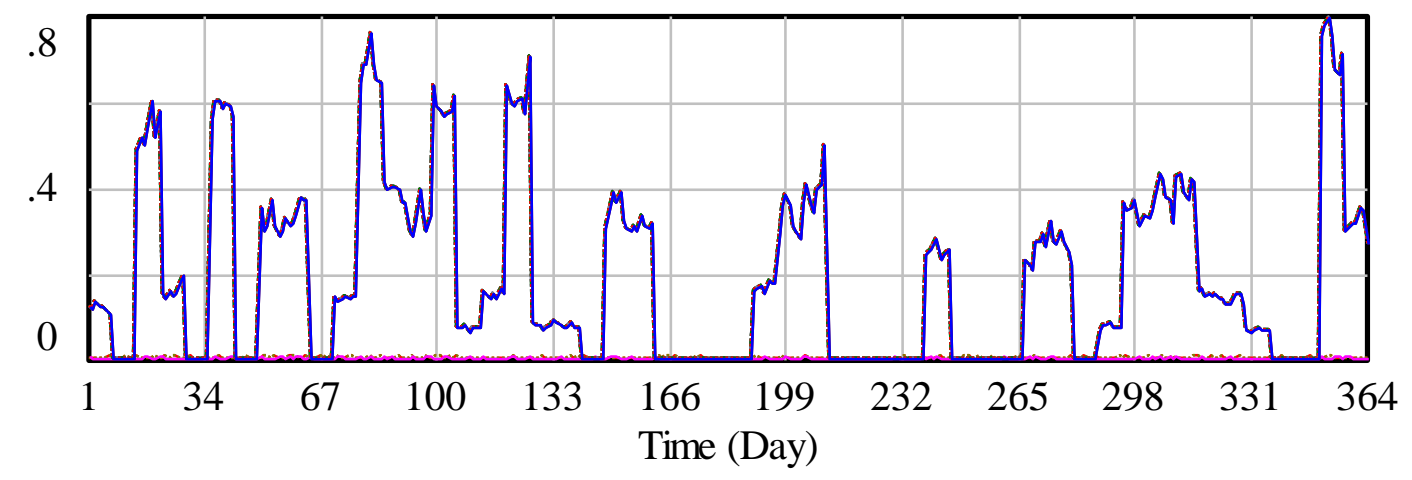

Larva survival rate : run 34

Larva survival rate : run 33

Larva survival rate : run 32

Larva survival rate : run 31

Larva survival rate : run 30

Larva survival rate : run 29

Larva survival rate : run 28

Larva survival rate : run 27

Larva survival rate : run 26

Larva survival rate : run 25

Figure 29: Larva survival rate simulation

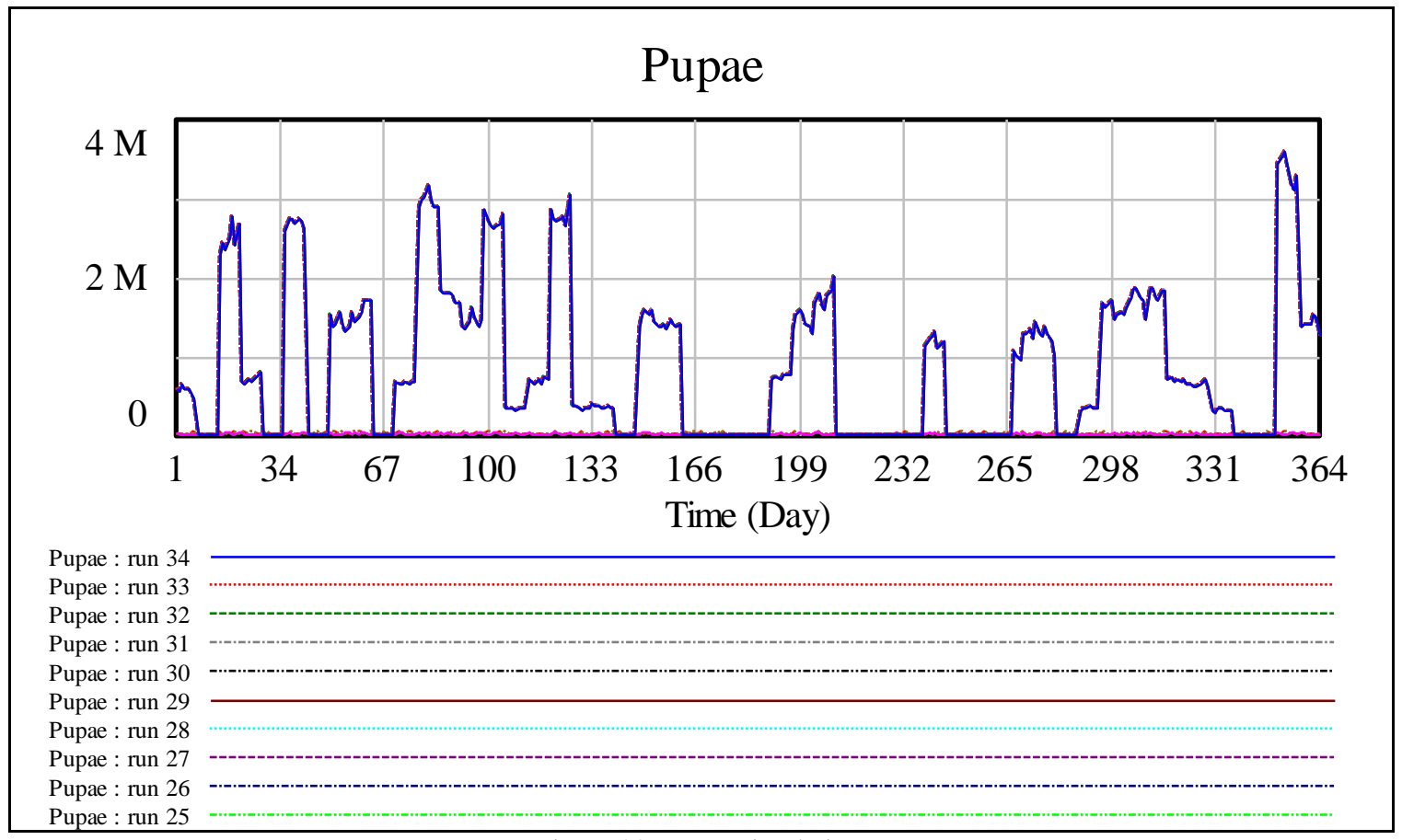

Figure 30: Pupae simulation 


\section{Pupae development rate}

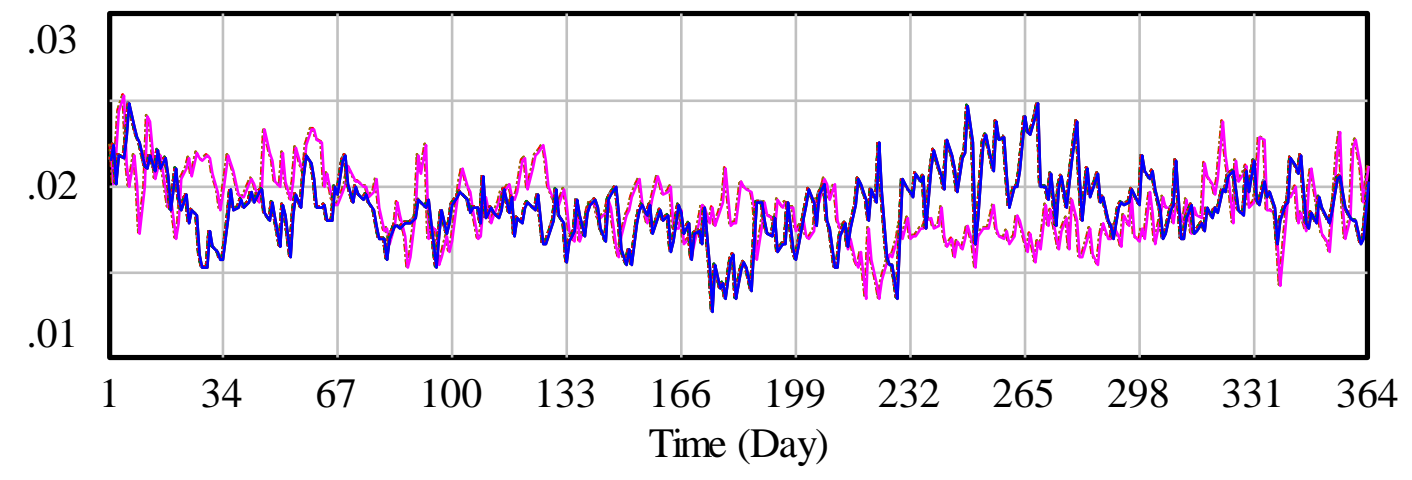

Pupae development rate : run 34

Pupae development rate : run 33

Pupae development rate : run 32

Pupae development rate : run 31

Pupae development rate : run 30

Pupae development rate : run 29

Pupae development rate : run 28

Pupae development rate : run 27

Pupae development rate : run 26

Pupae development rate : run 25

Figure 31: Pupae development rate simulation

\section{Pupae survival rate}

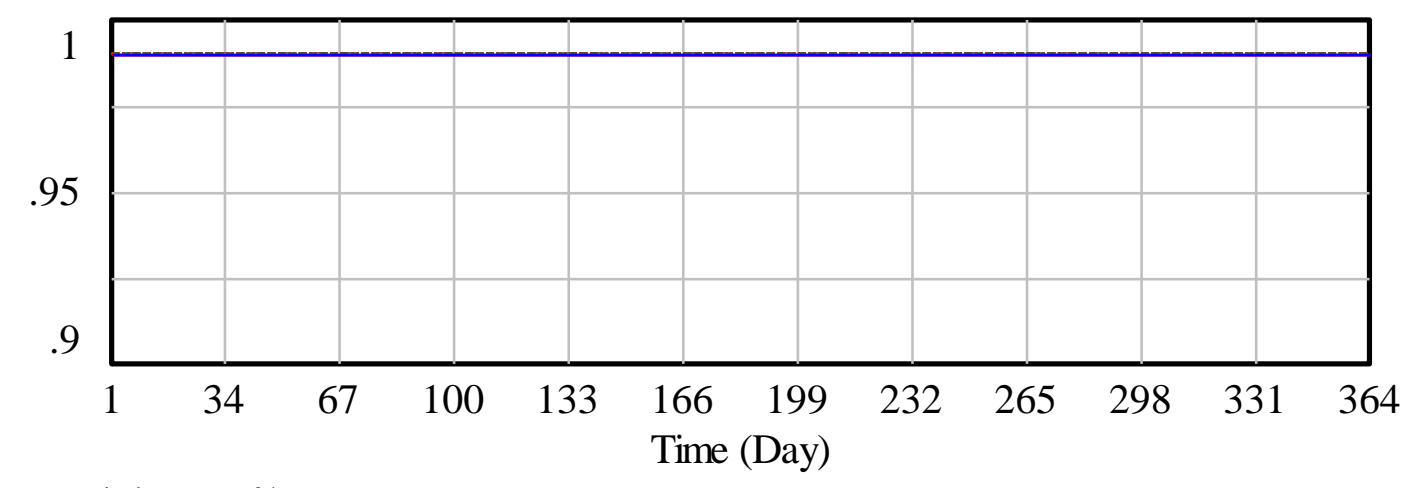

Pupae survival rate : run 34

Pupae survival rate : run 33

Pupae survival rate : run 32

Pupae survival rate : run 31

Pupae survival rate : run 30

Pupae survival rate : run 29

Pupae survival rate : run 28

Pupae survival rate : run 27

Pupae survival rate : run 26

Pupae survival rate : run 25

Figure 32: Pupae survival rate simulation 


\section{Emerging adult}

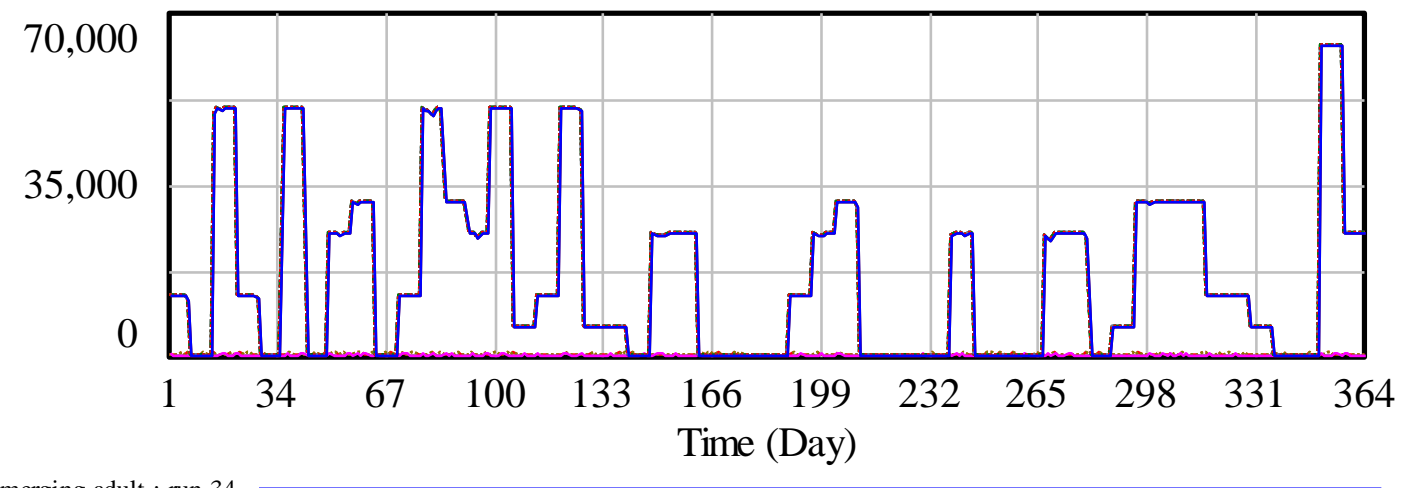

Emerging adult : run 34

Emerging adult : run 33

Emerging adult : run 32

Emerging adult : run 31

Emerging adult : run 30

Emerging adult : run 29

Emerging adult : run 28

Emerging adult : run 27

Emerging adult : run 26

Emerging adult : run 25

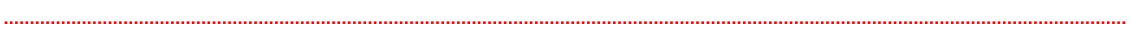

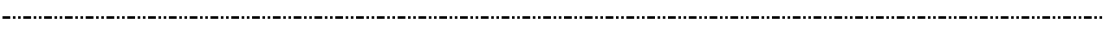

Figure 33: Emerging adult simulation

Female adult

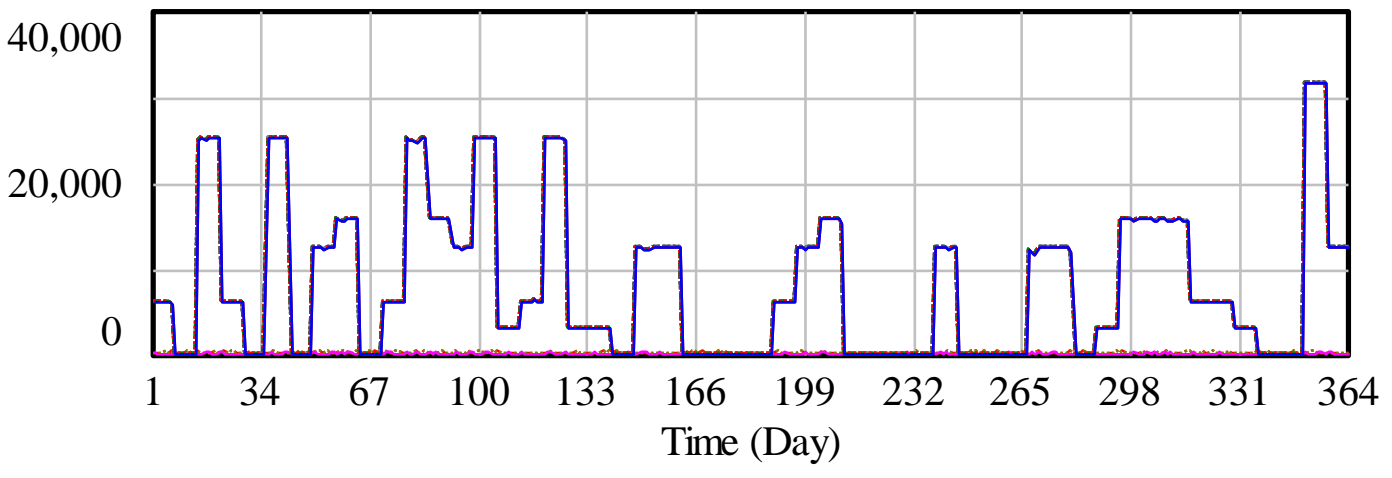

Female adult : run 34

Female adult : run 33

Female adult : run 32

Female adult : run 31

Female adult : run 30

Female adult : run 29

Female adult : run 28

Female adult : run 27

Female adult : run 26

Female adult : run 25

Figure 34: Female adult simulation 


\section{Fertilized adult}

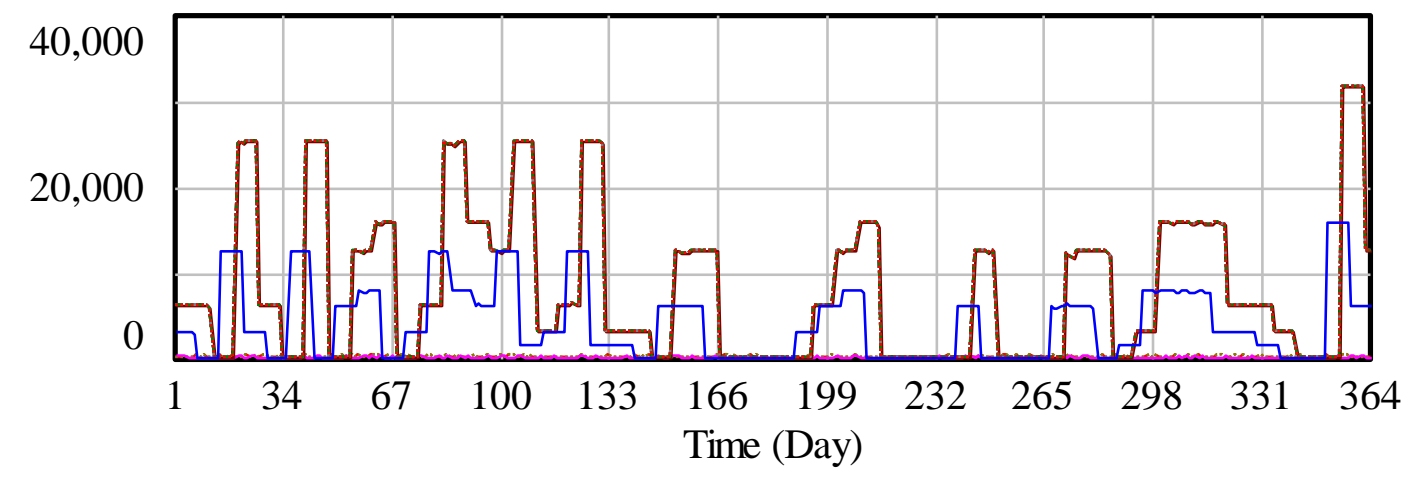

Fertilized adult : run 34

Fertilized adult : run 33

Fertilized adult : run 32

Fertilized adult : run 31

Fertilized adult : run 30

Fertilized adult : run 29

Fertilized adult : run 28

Fertilized adult : run 27

Fertilized adult : run 26

Fertilized adult : run 25

Figure 35: Fertilized adult simulation

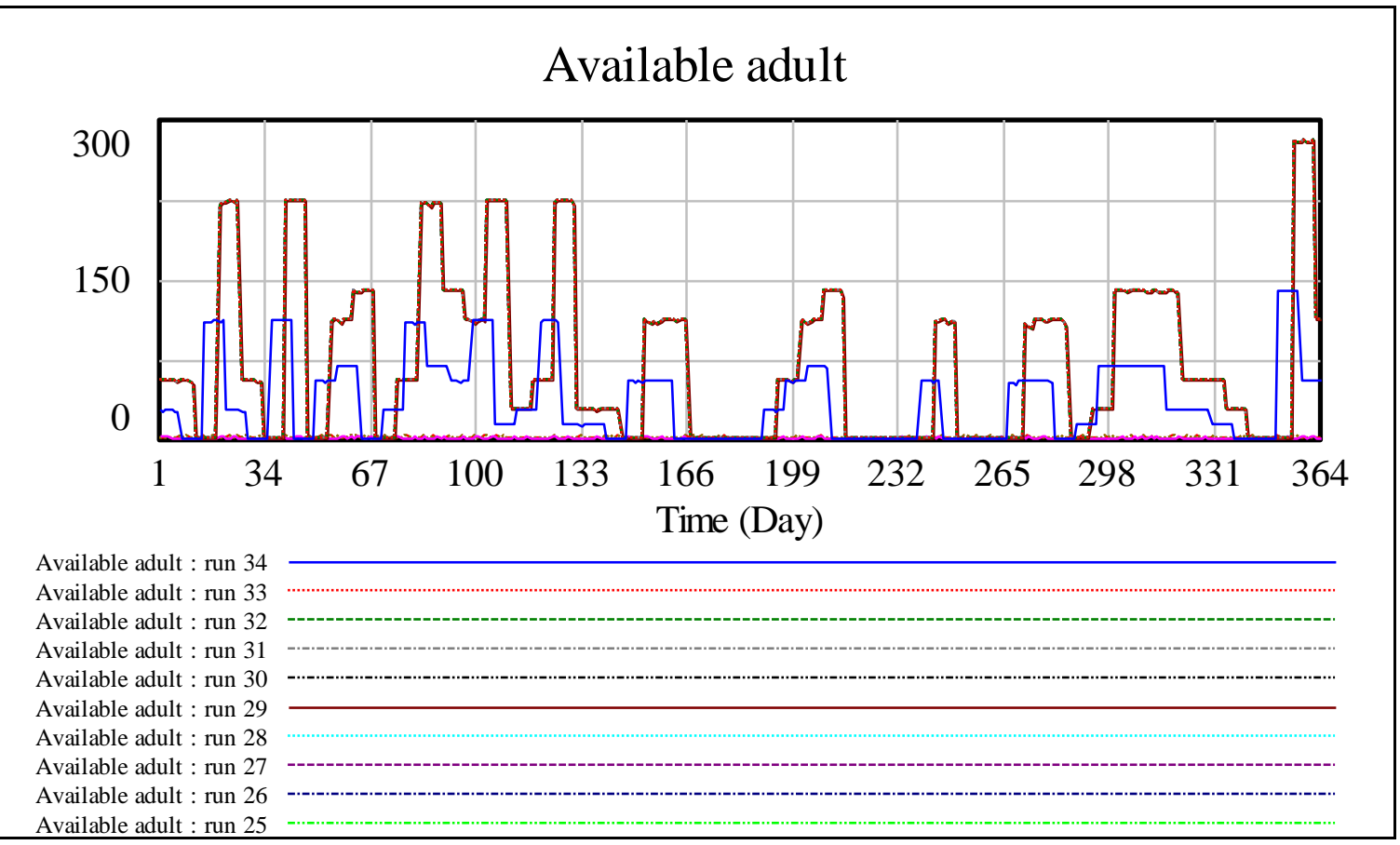

Figure 36: Available adult simulation 


\section{EIP progress rate}

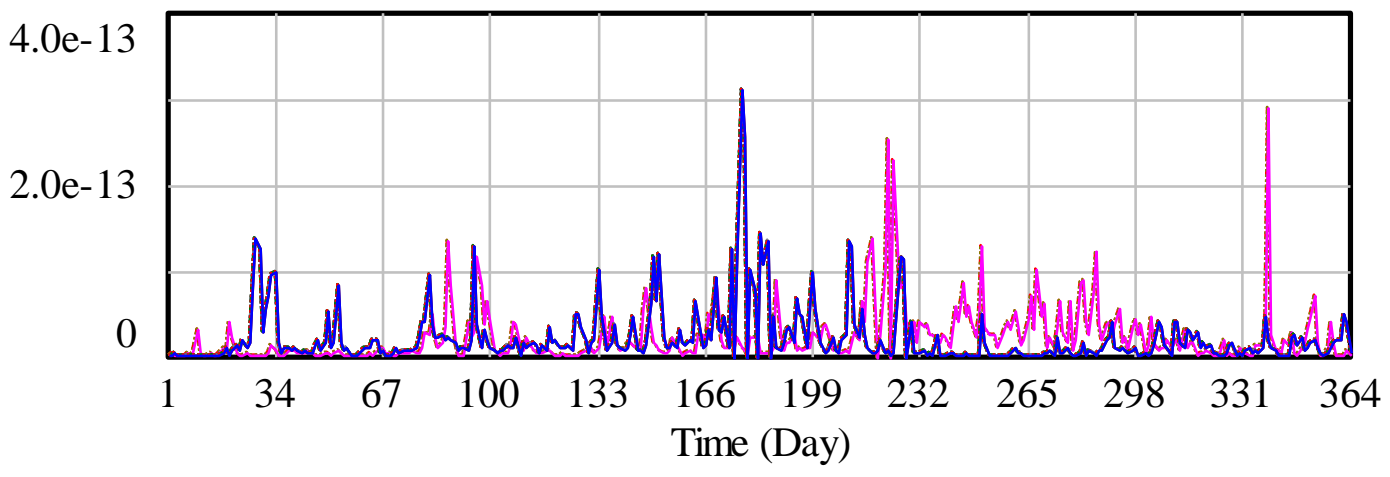

EIP progress rate : run 34

EIP progress rate : run 33

EIP progress rate : run 32

EIP progress rate : run 31

EIP progress rate : run 29

EIP progress rate : run 28

EIP progress rate : run 27

EIP progress rate : run 26

EIP progress rate : run 25

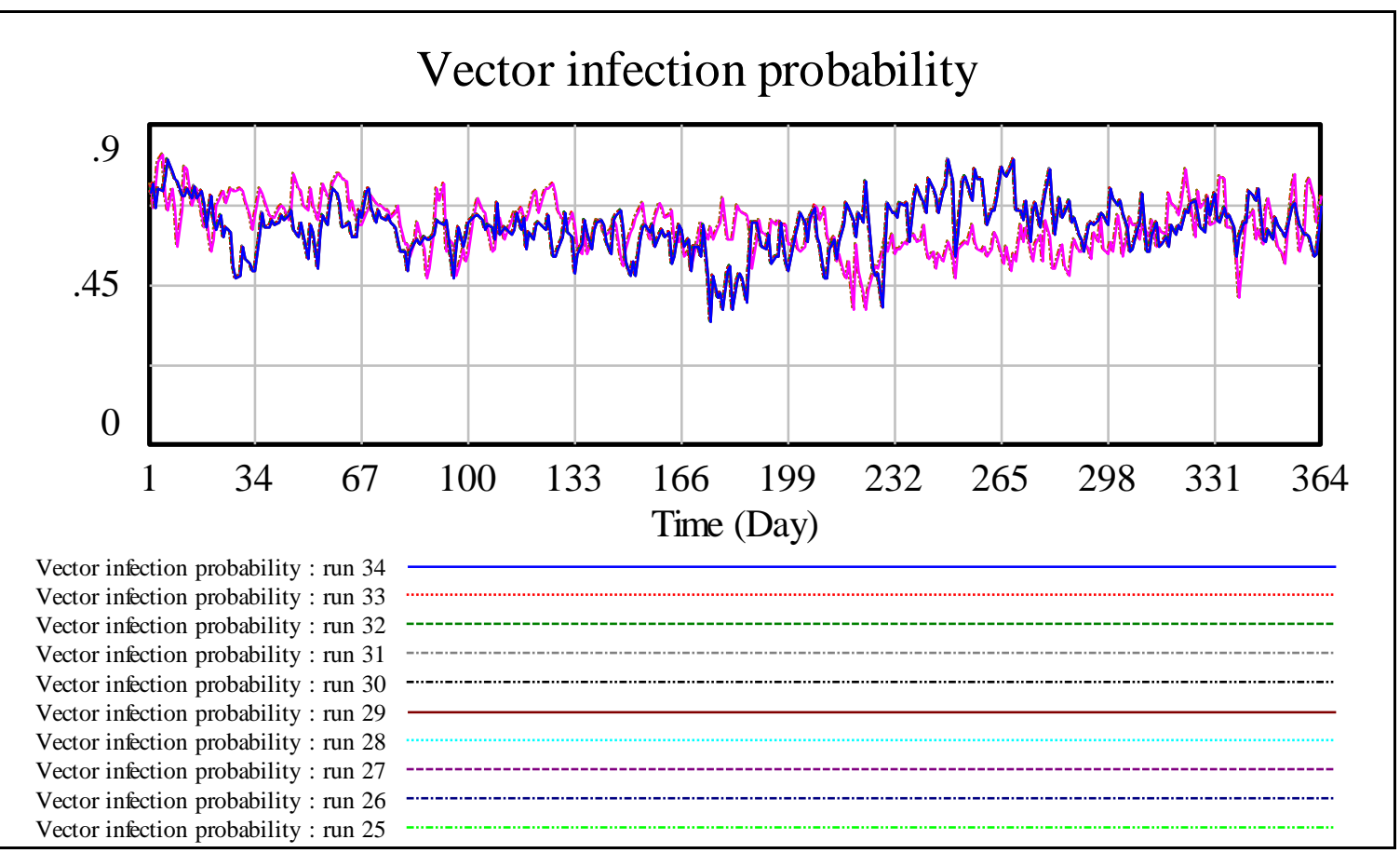

Figure 38: Vector infection probability simulation 


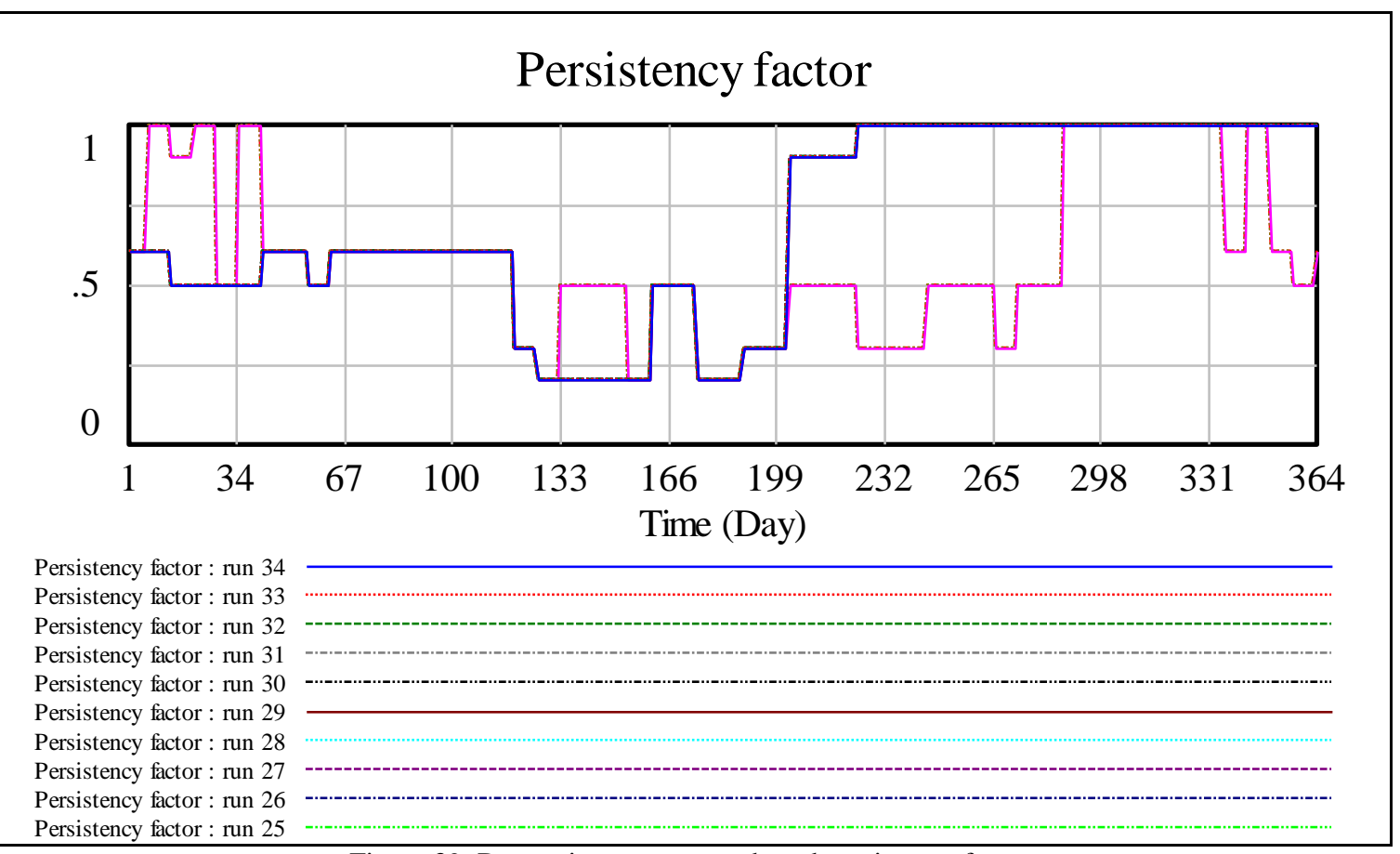

Figure 39: Dew point temperature based persistency factor simulation

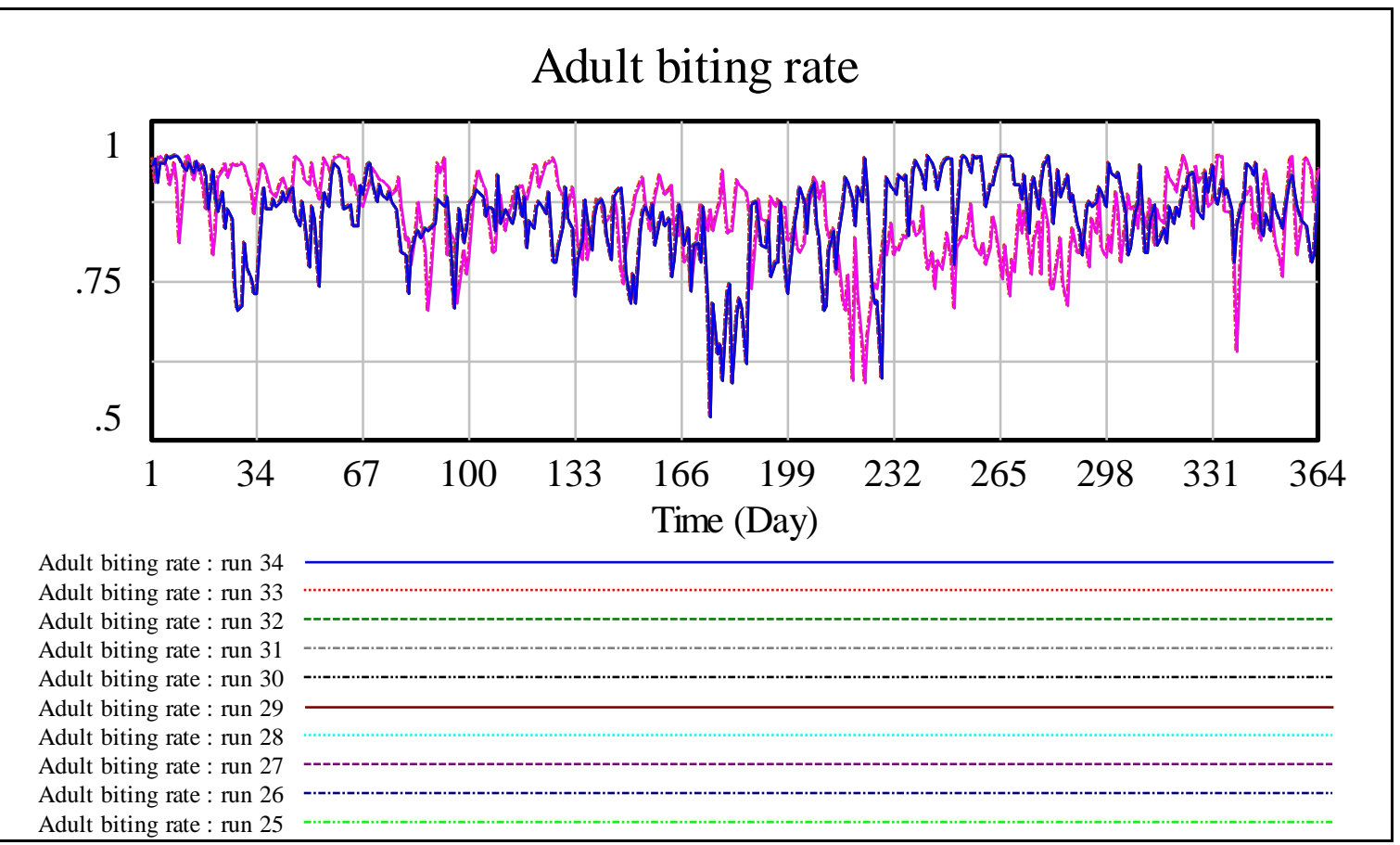

Figure 40: Adult biting rate simulation 


\section{Infectious adult}

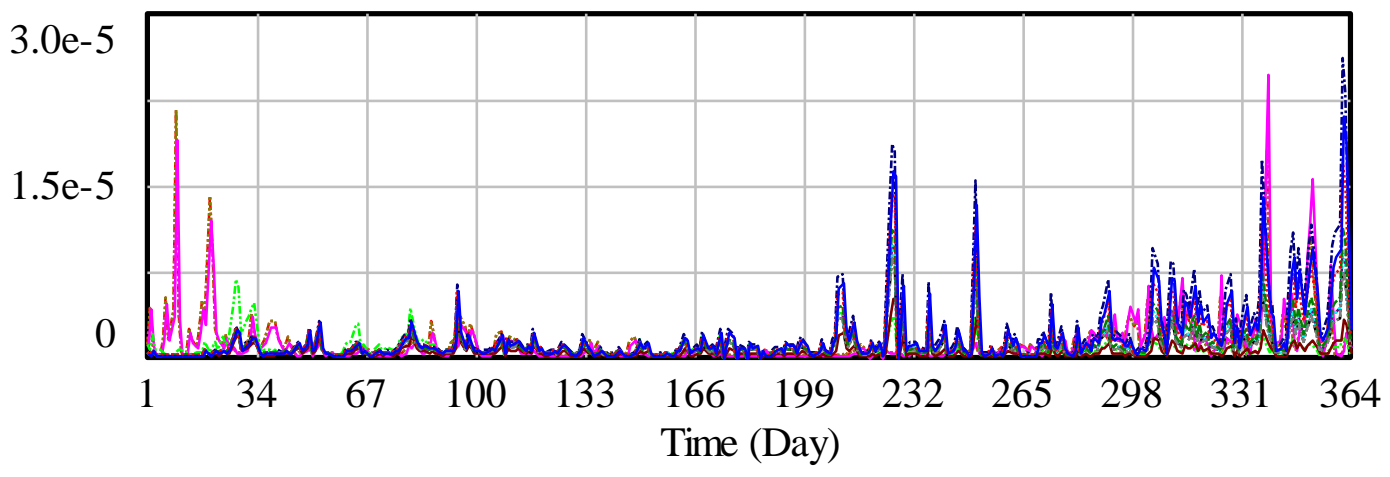

Infectious adult : run 34

Infectious adult : run 33

Infectious adult : run 32

Infectious adult : run 31

Infectious adult : run 30

Infectious adult : run 29

Infectious adult : run 28

Infectious adult : run 27

Infectious adult : run 26

Infectious adult : run 25

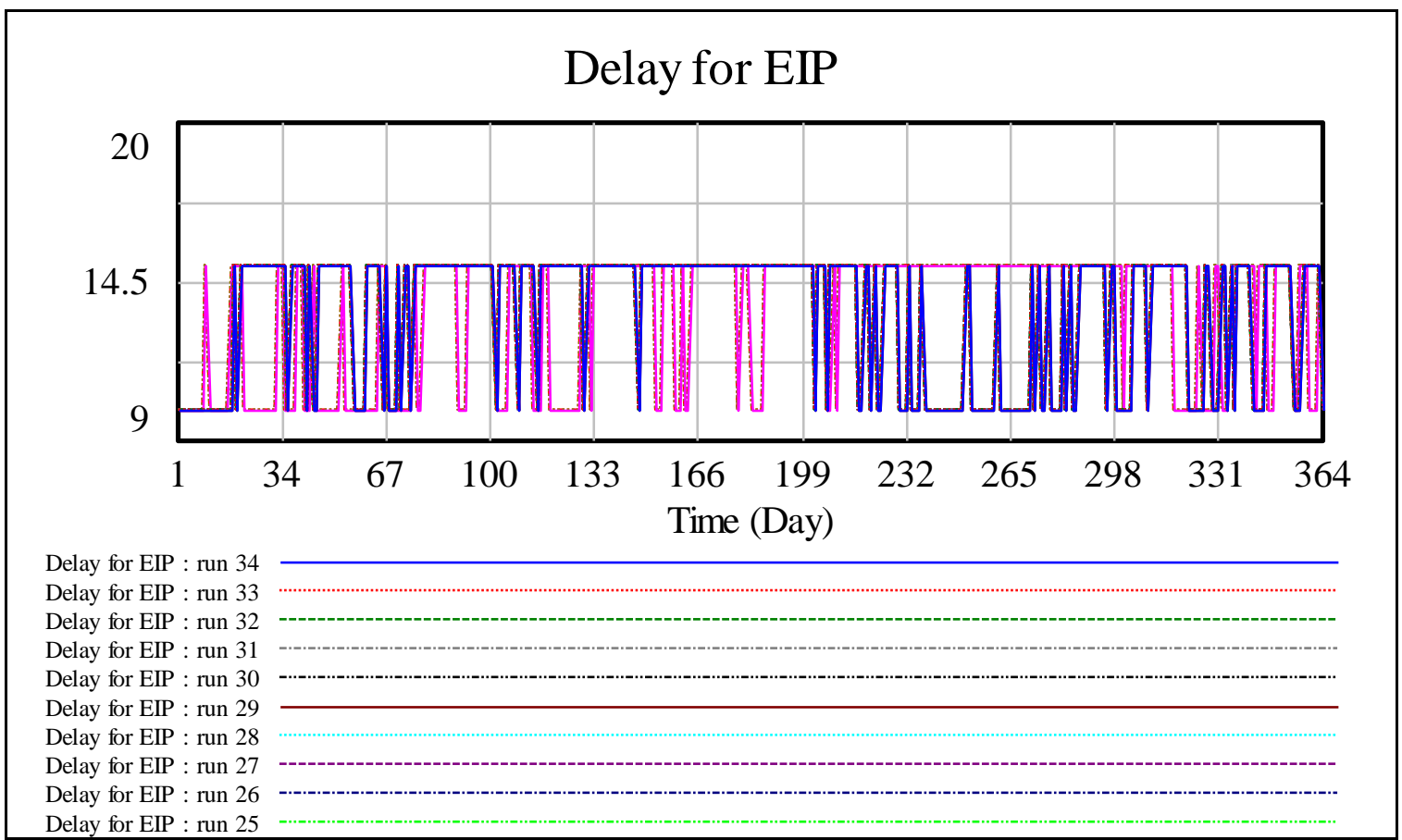

Figure 42: Delay for EIP simulation 


\section{Effective infectious adult}

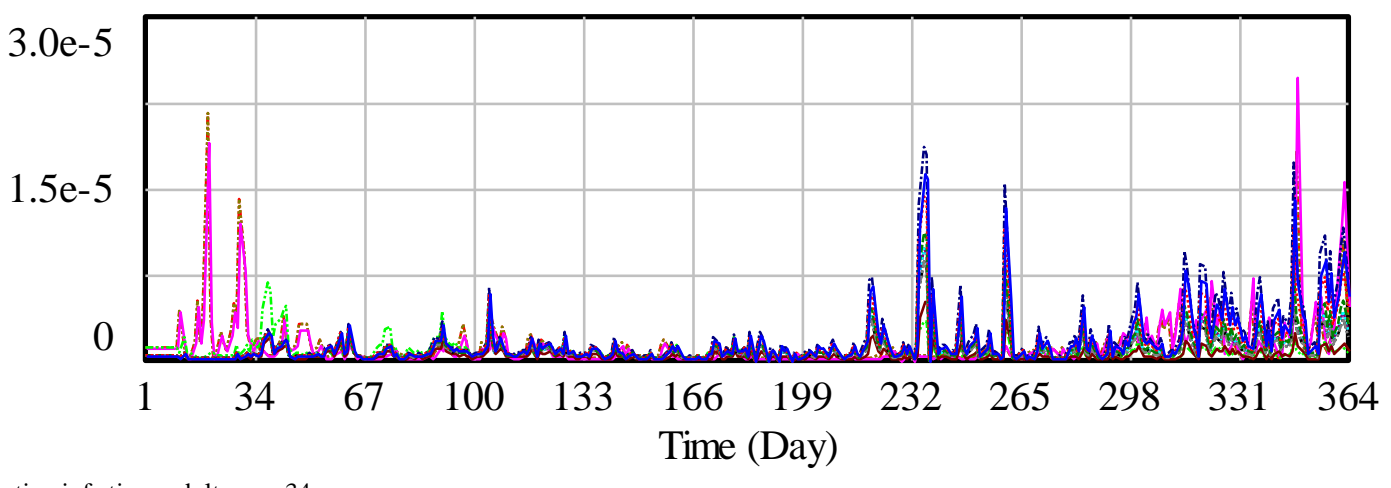

Effective infectious adult : run 34

Effective infectious adult : run 33

Effective infectious adult : run 32

Effective infectious adult : run 31

Effective infectious adult : run 30

Effective infectious adult : run 29

Effective infectious adult : run 28

Effective infectious adult : run 27

Effective infectious adult : run 26

Effective infectious adult : run 25

Figure 43: Effective infectious adult simulation

\section{Host infection probability}

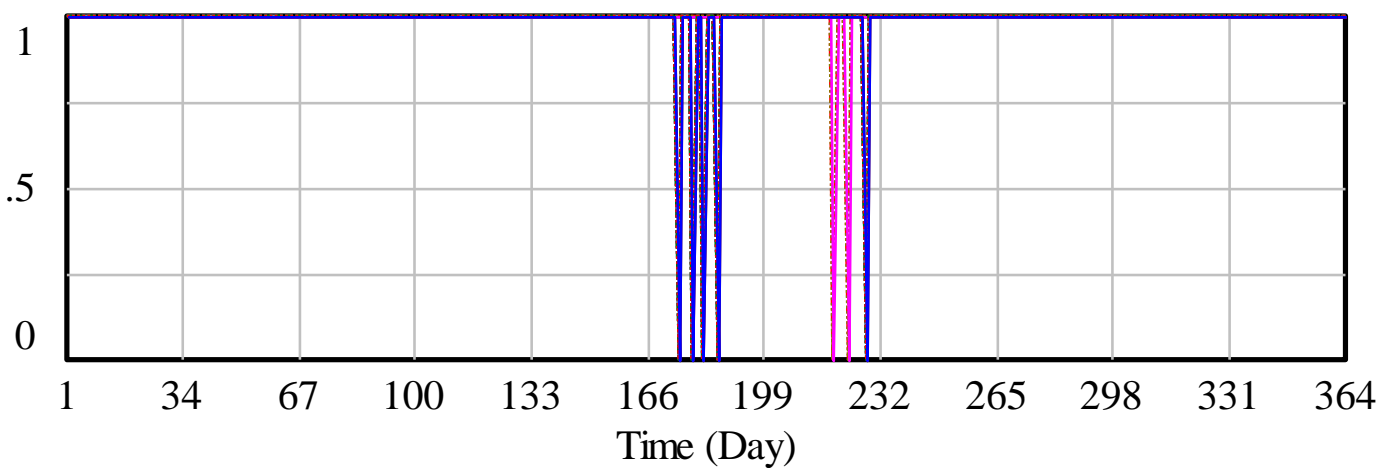

Host infection probability : run 34

Host infection probability : run 33

Host infection probability : run 32

Host infection probability : run 31

Host infection probability : run 30

Host infection probability : run 29

Host infection probability : run 28

Host infection probability : run 27

Host infection probability : run 26

Host infection probability : run 25

Figure 44: Host infection probability simulation 
Host with bloodmeal

6

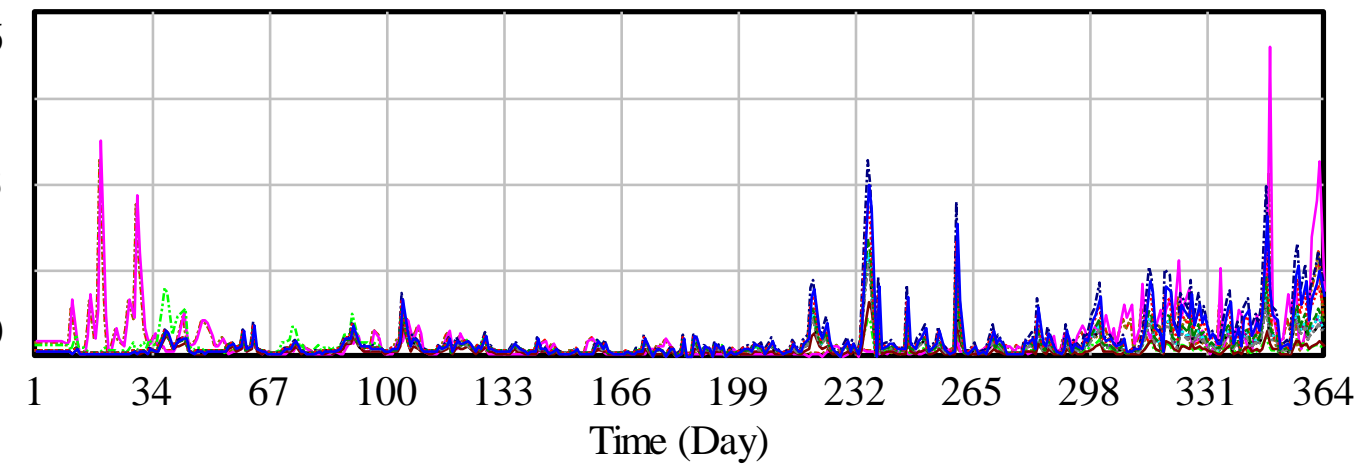

Host with bloodmeal : run 34

Host with bloodmeal : run 33

Host with bloodmeal : run 32

Host with bloodmeal : run 31

Host with bloodme

Host with bloodmeal : run 28

Host with bloodmeal : run 27

Host with bloodmeal : run 26

Host with bloodmeal : run 25

Figure 45: Host with bloodmeal simulation

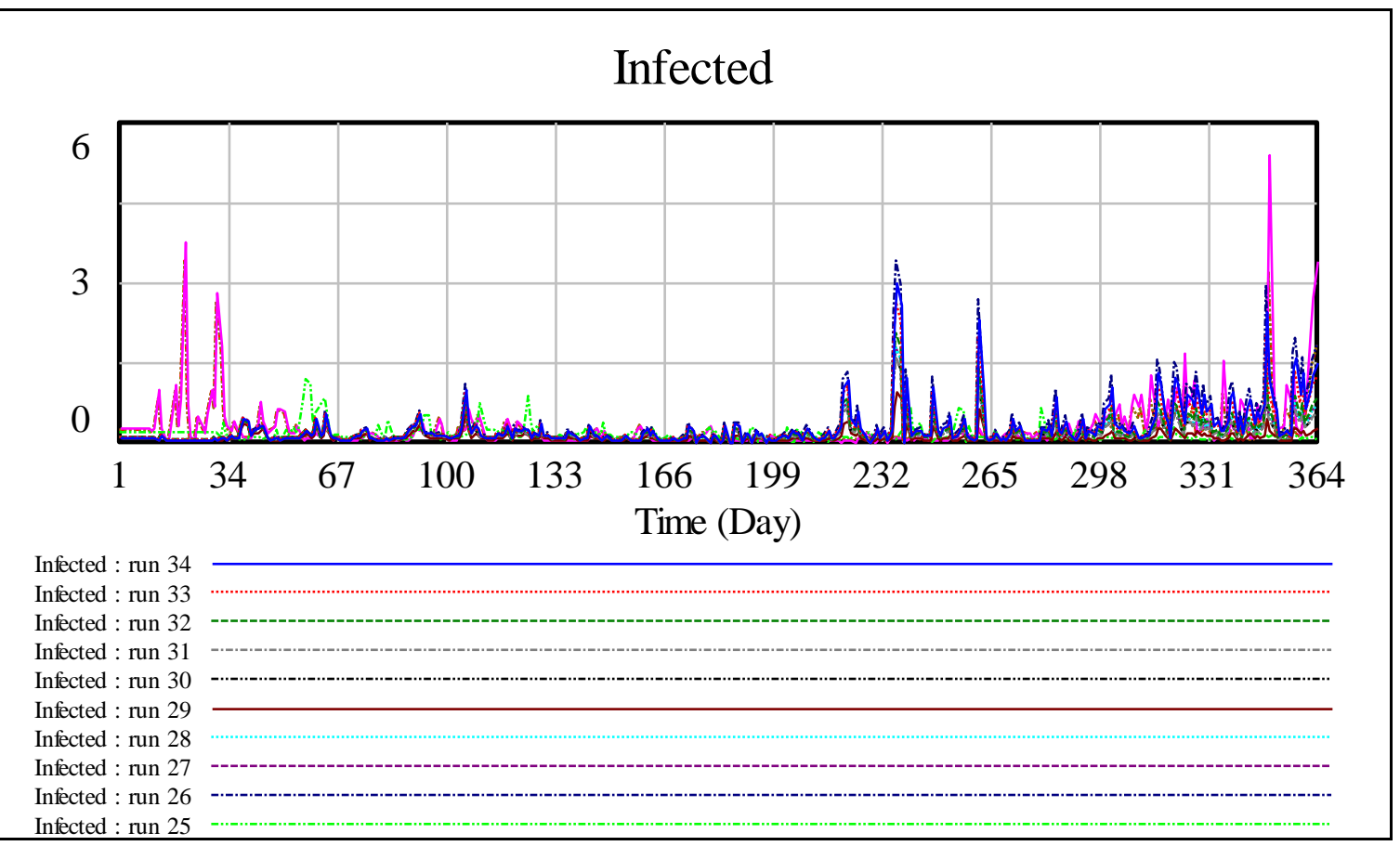

Figure 46: Infected simulation 


\section{Infectious host}

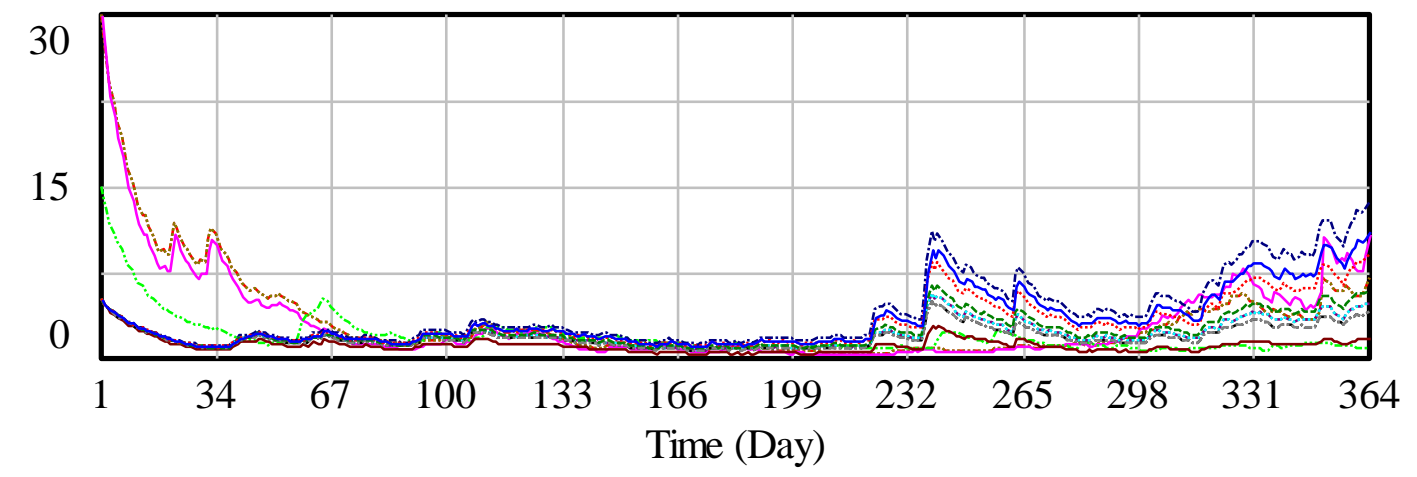

Infectious host : run 34

Infectious host : run 33

Infectious host : run 32

Infectious host : run 31

Infectious host : run 30

Infectious host : run 29

Infectious host : run 28

Infectious host : run 27

Infectious host : run 26

Infectious host : run 25

Figure 47: Infectious host simulation 


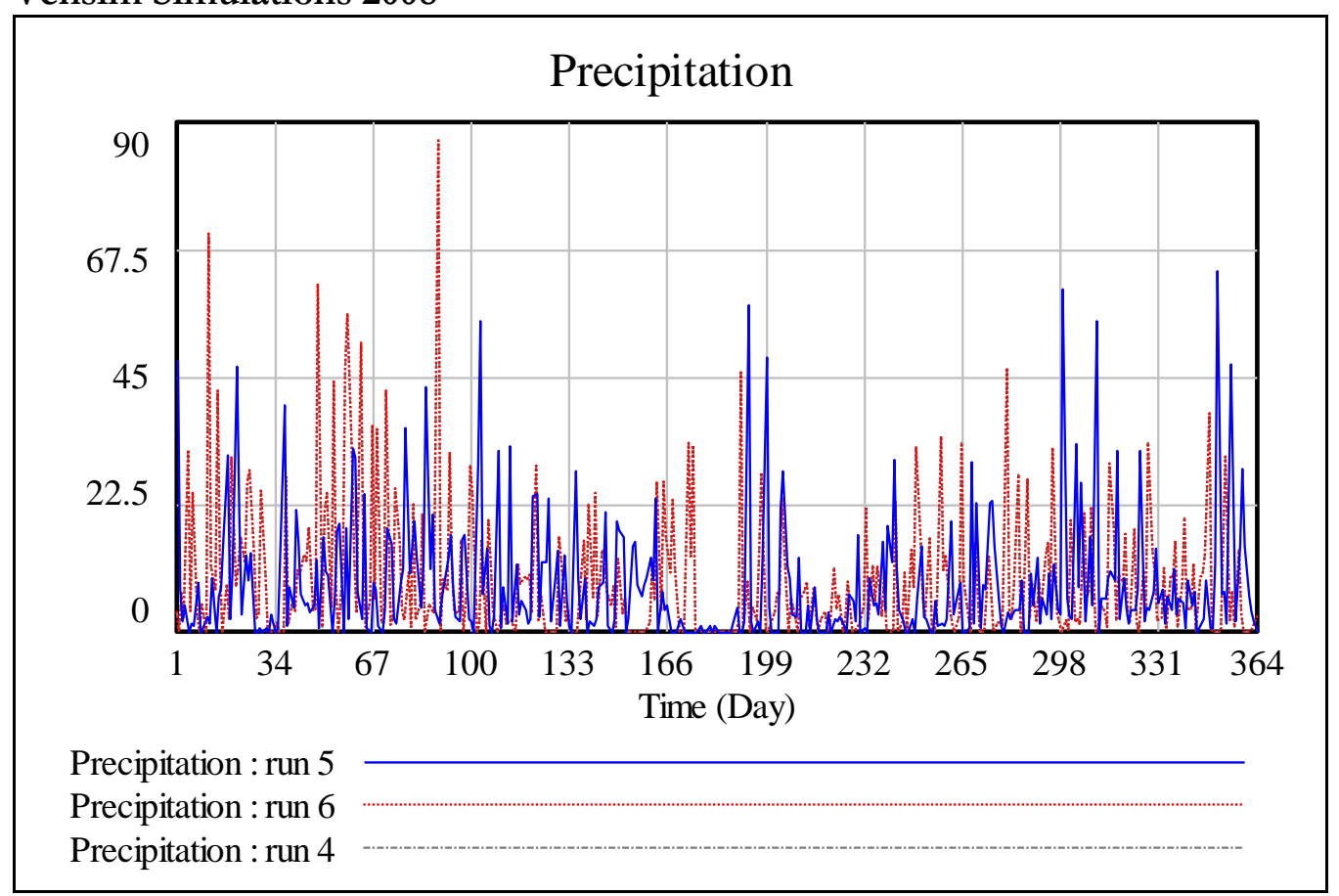

Figure 48: Precipitation simulation 2008

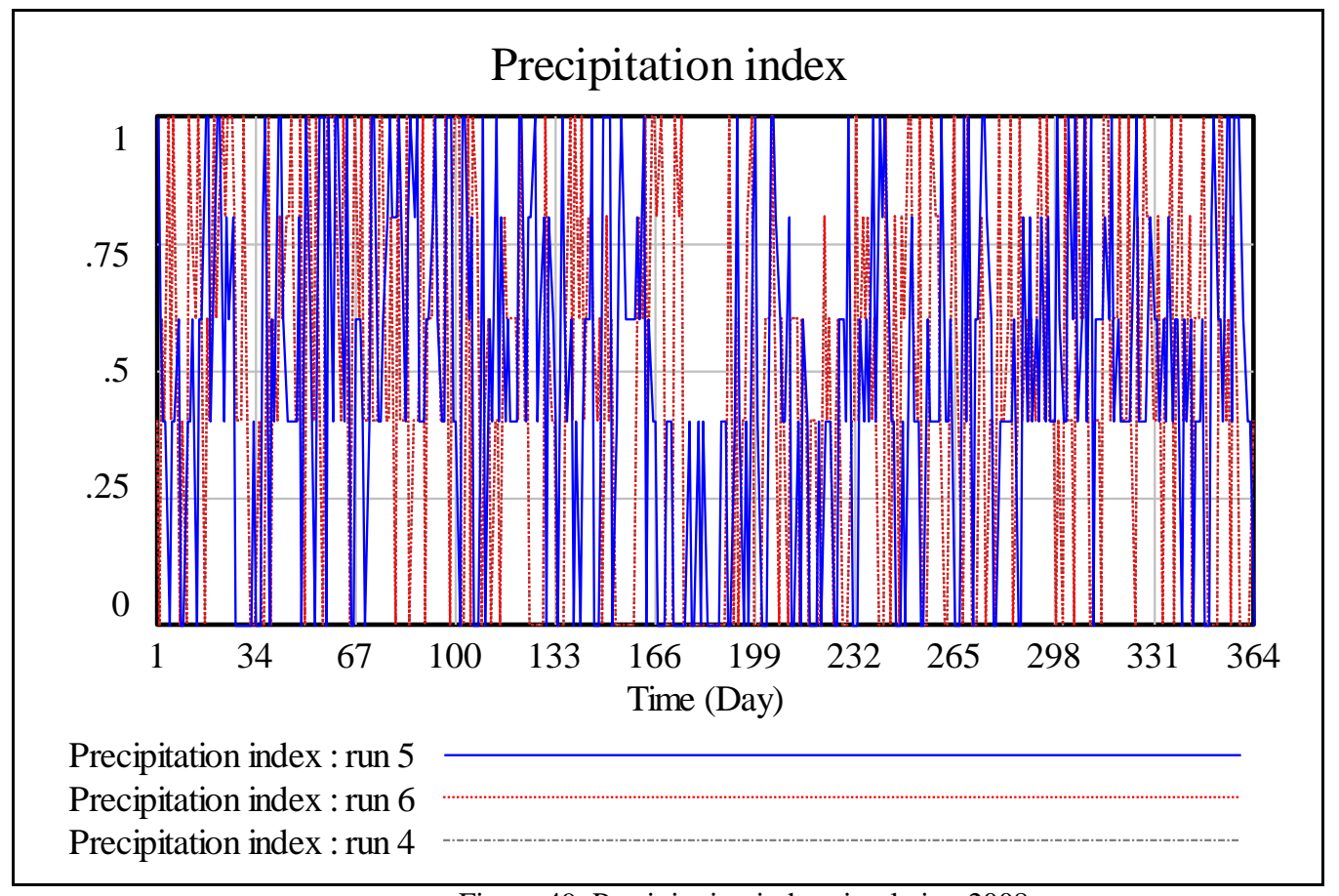

Figure 49: Precipitation index simulation 2008 


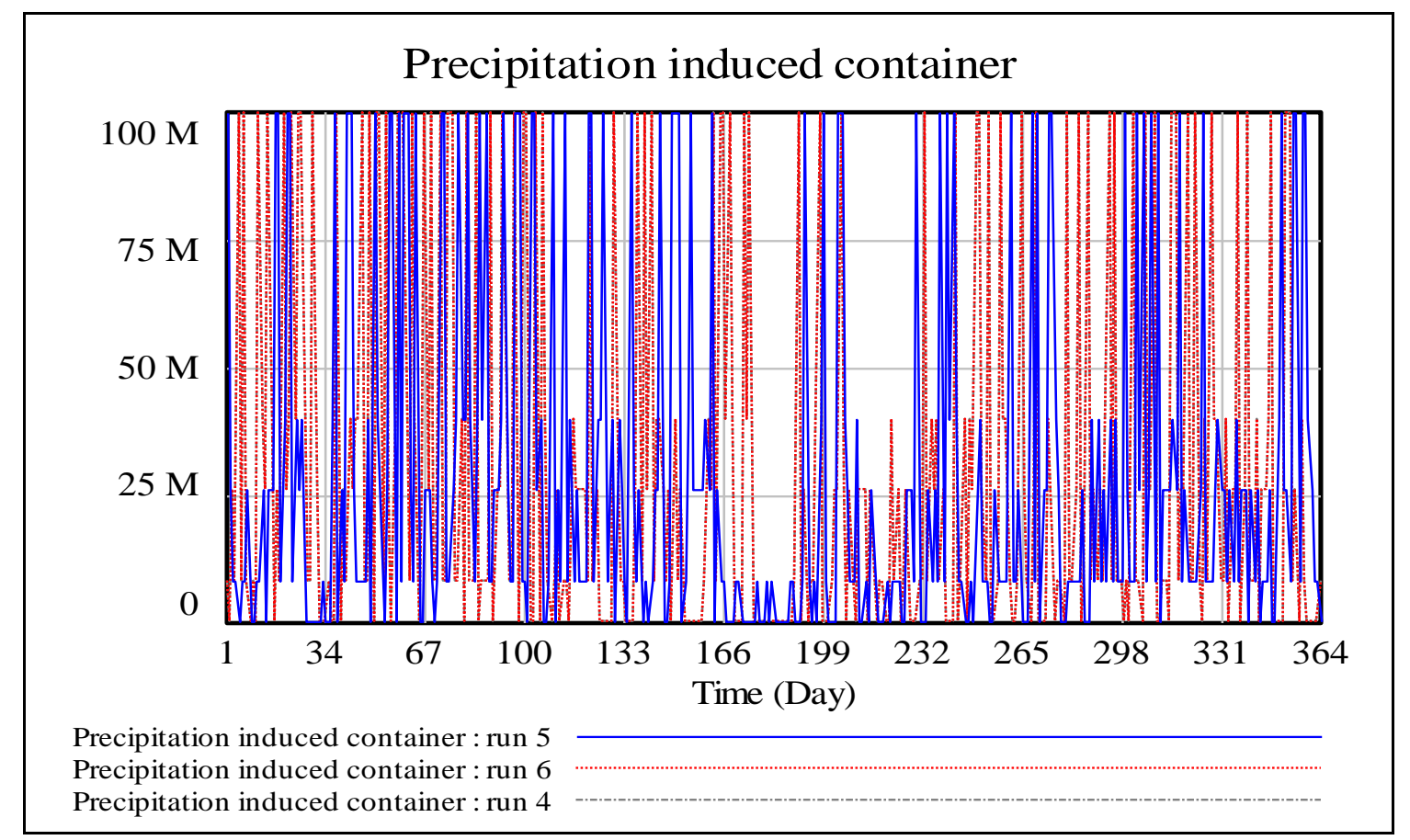

Figure 50: Precipitation induced container simulation 2008

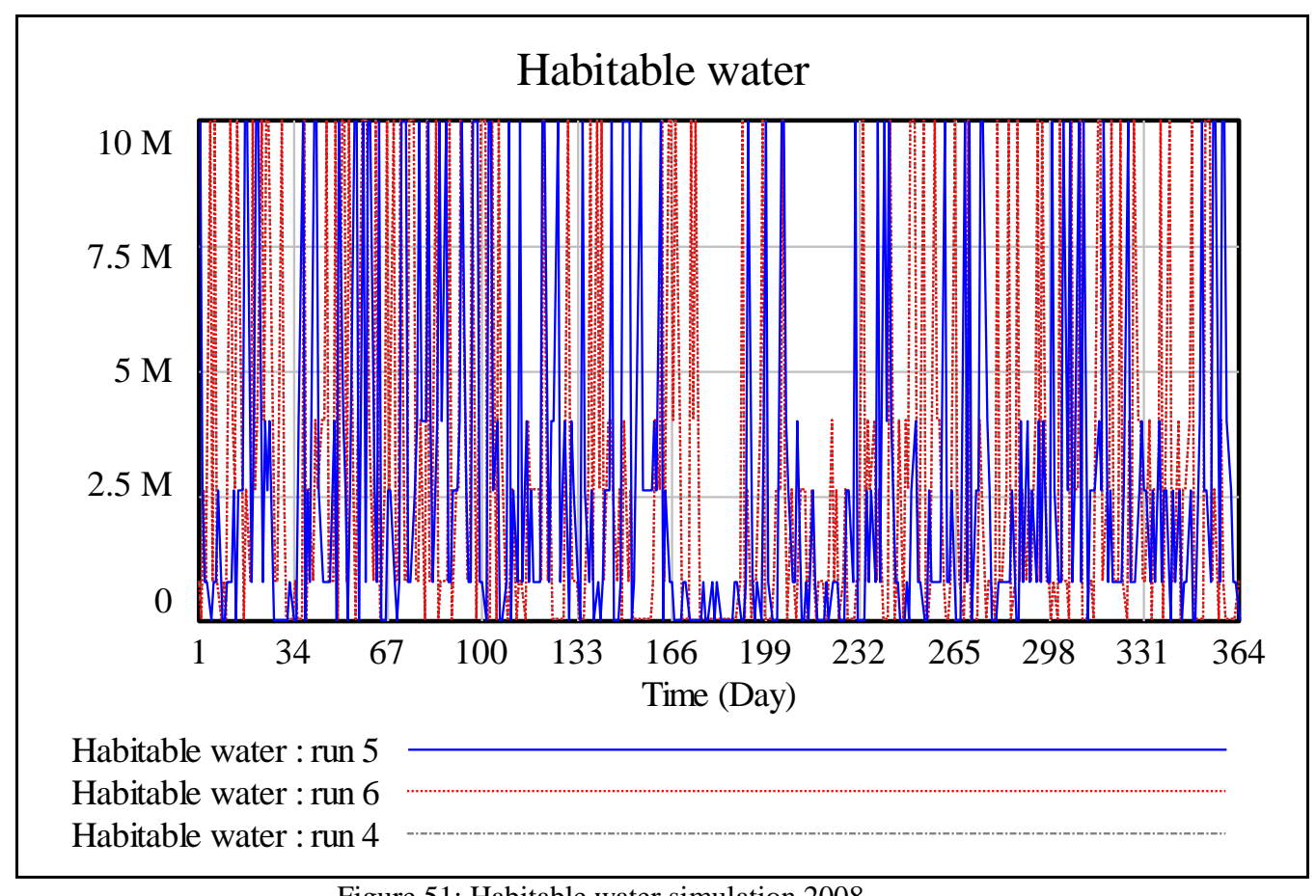

Figure 51: Habitable water simulation 2008 


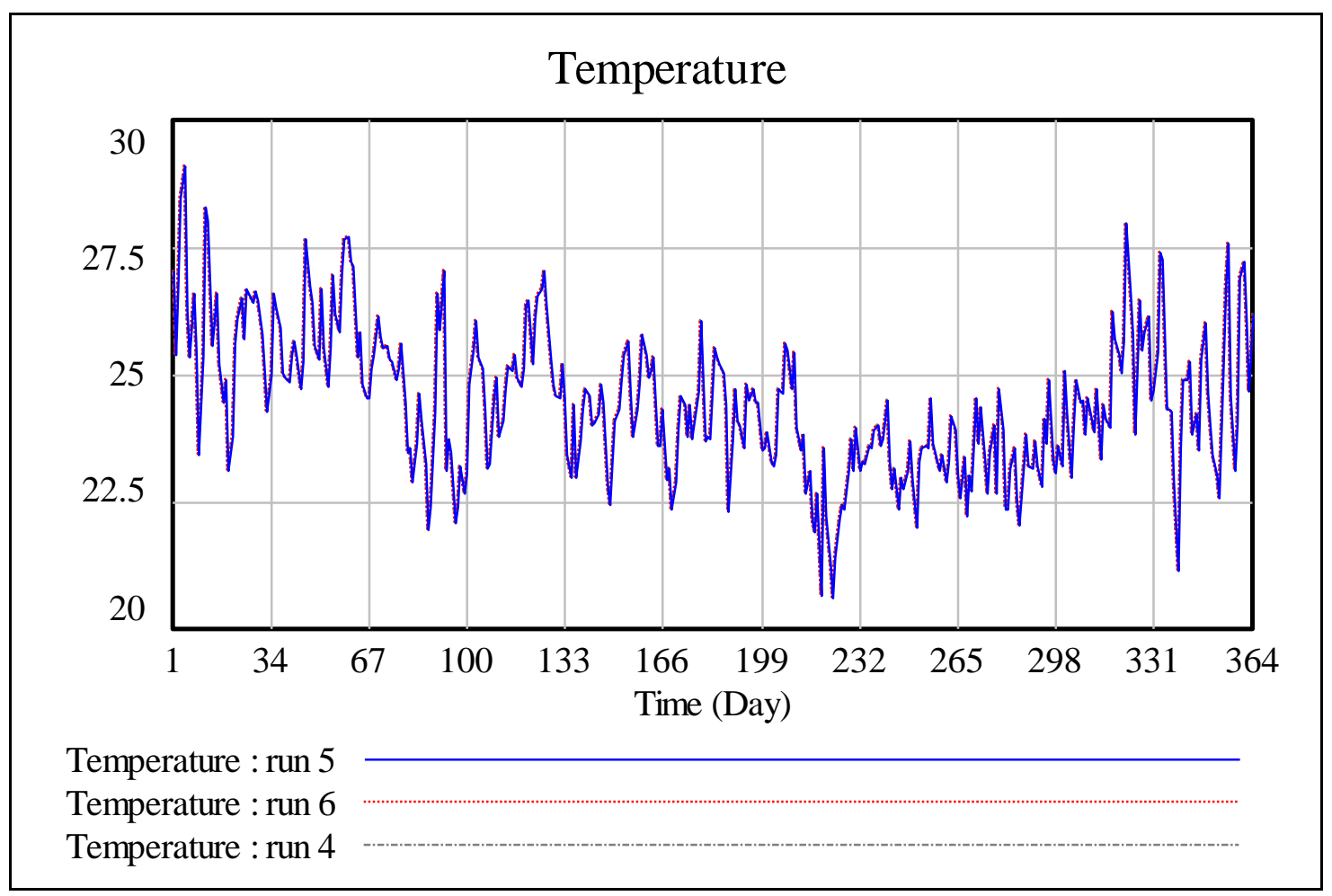

Figure 52: Temperature simulation 2008

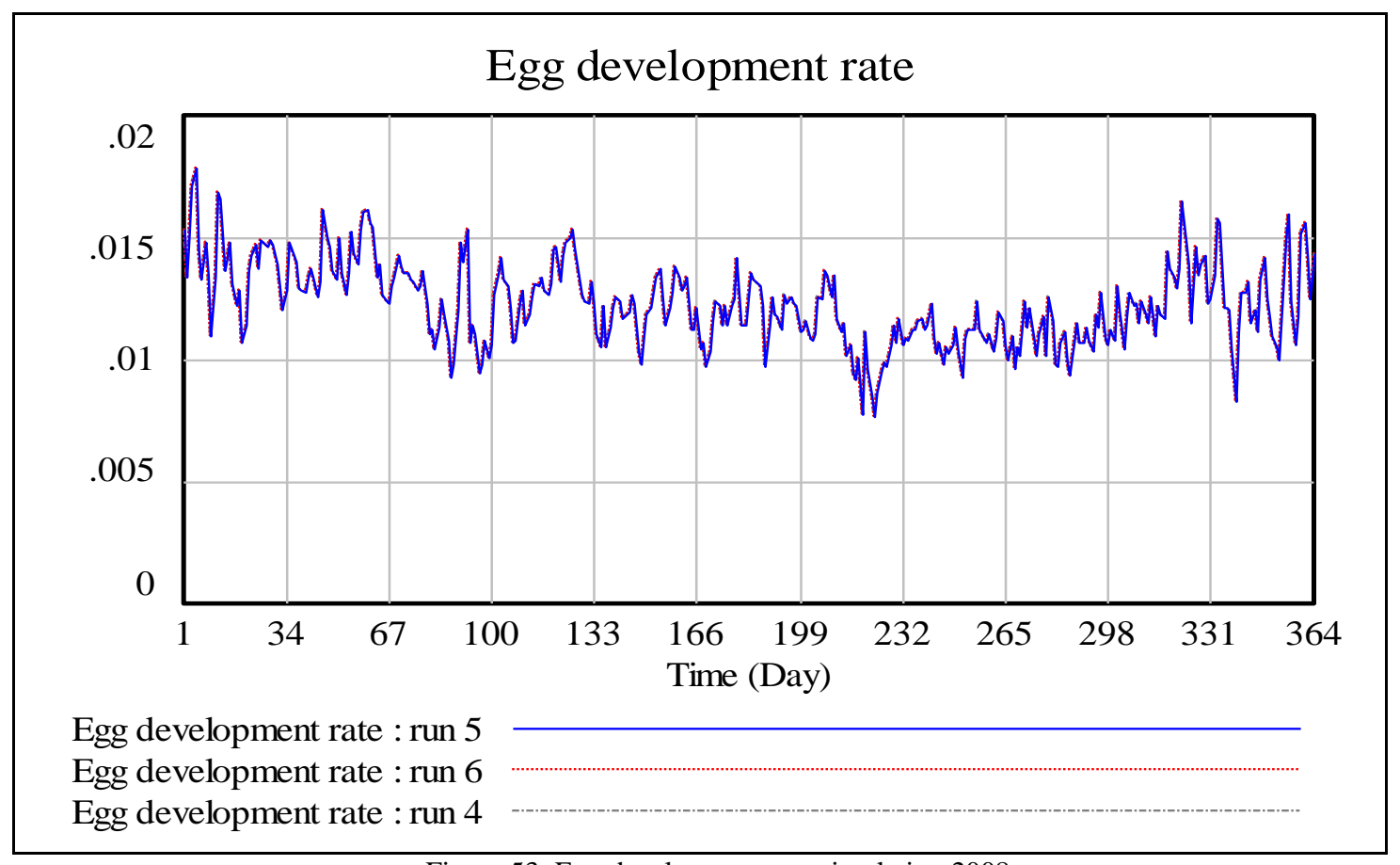

Figure 53: Egg development rate simulation 2008 


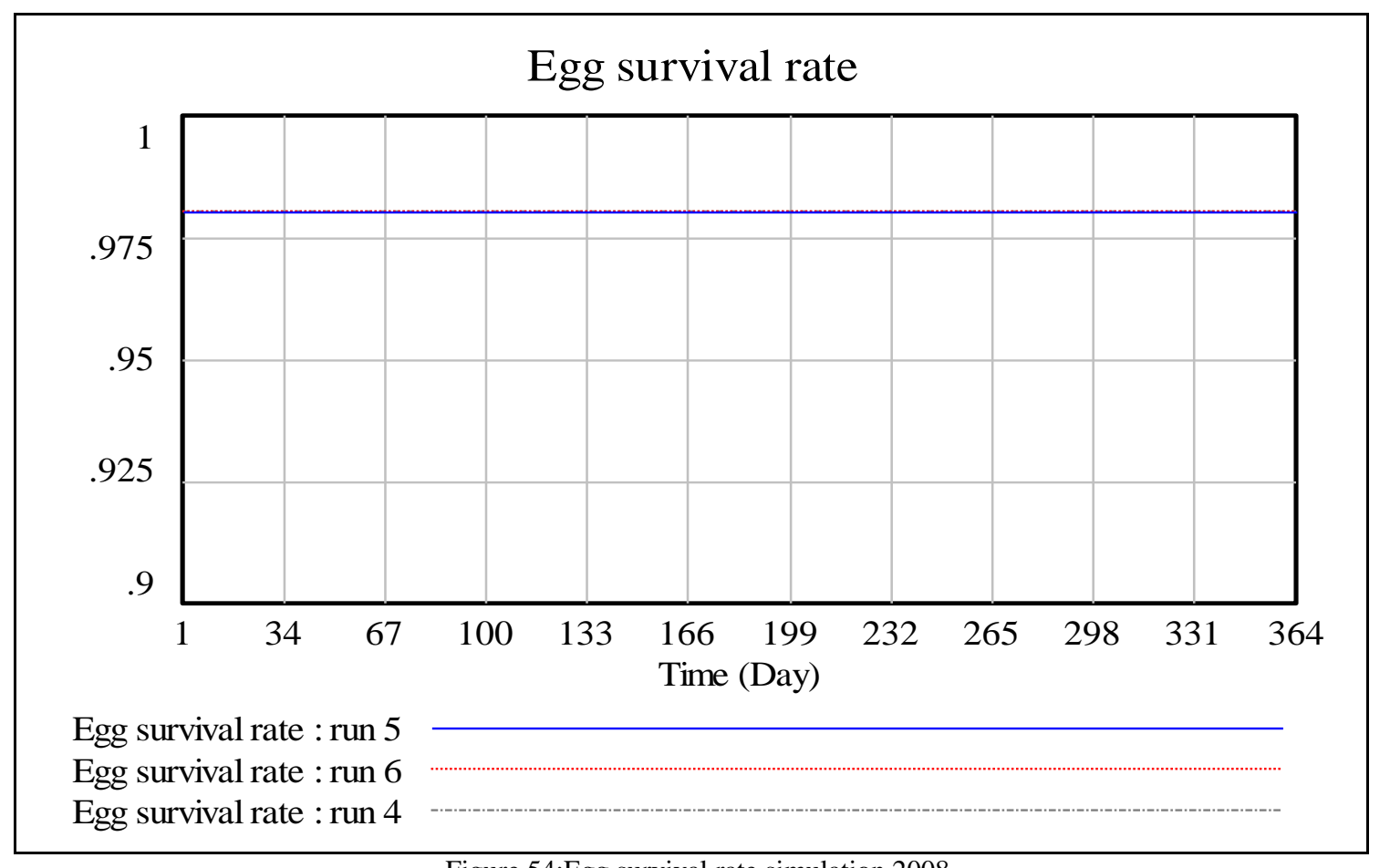

Figure 54:Egg survival rate simulation 2008

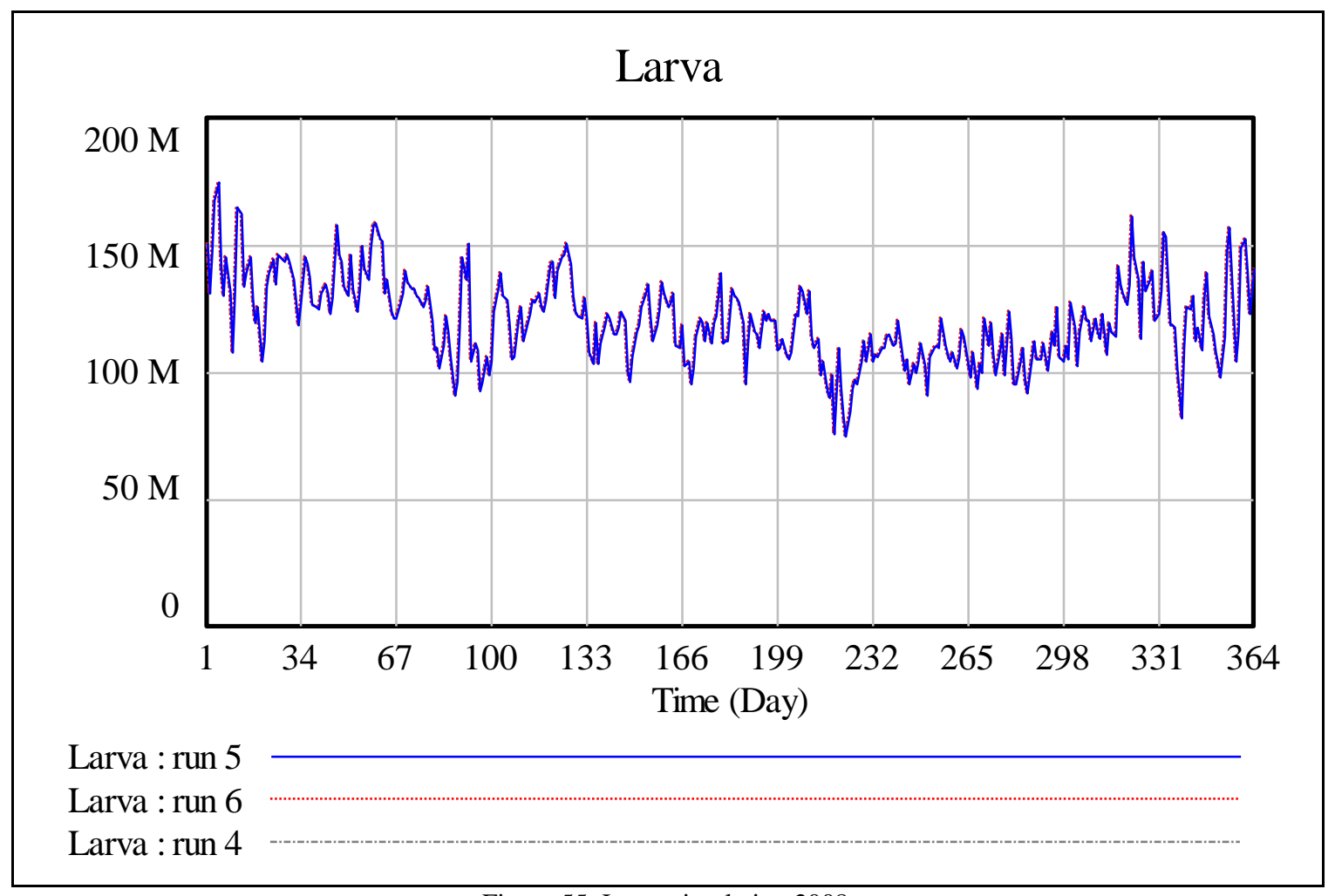

Figure 55: Larva simulation 2008 


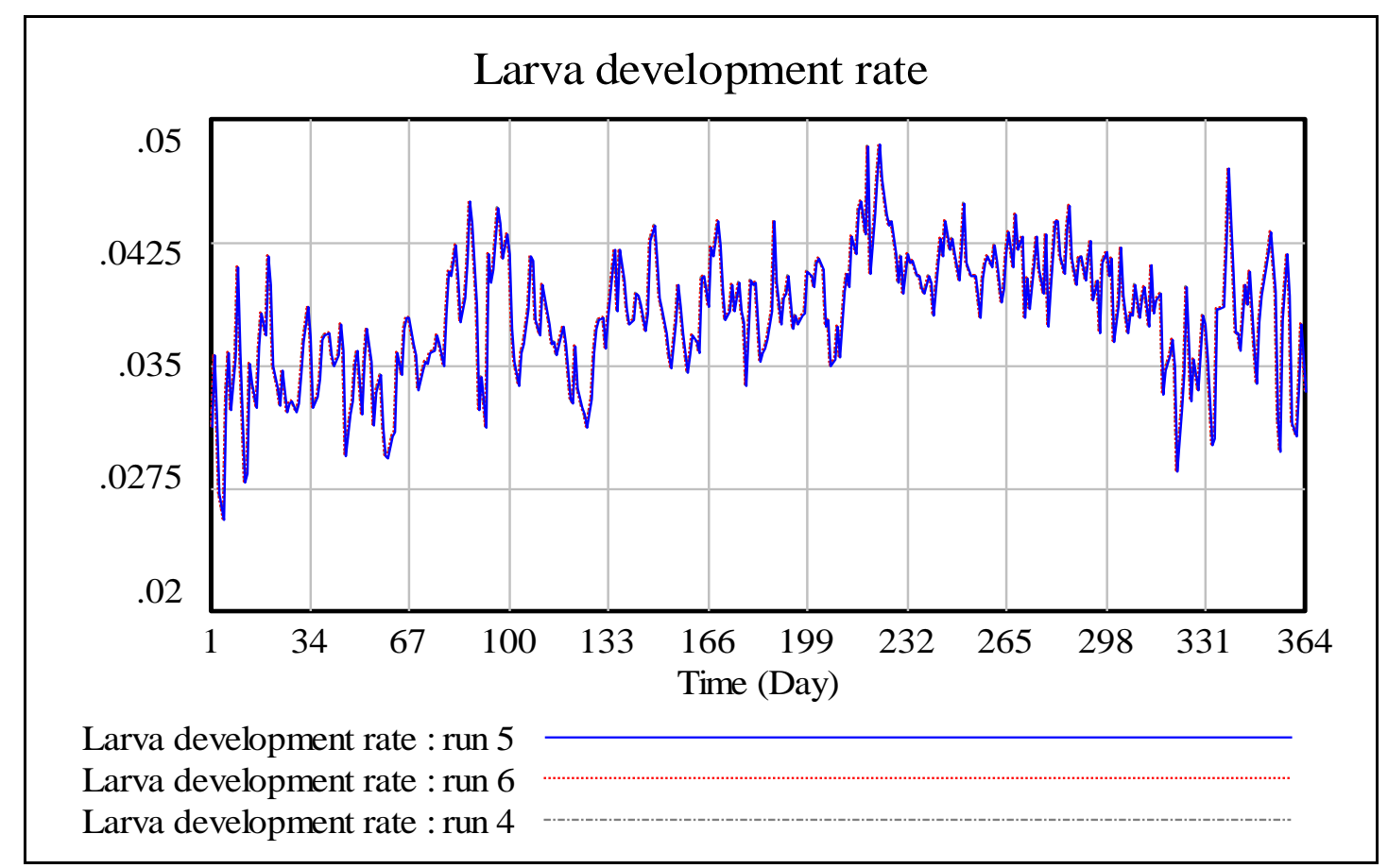

Figure 56: Larva development rate simulation 2008

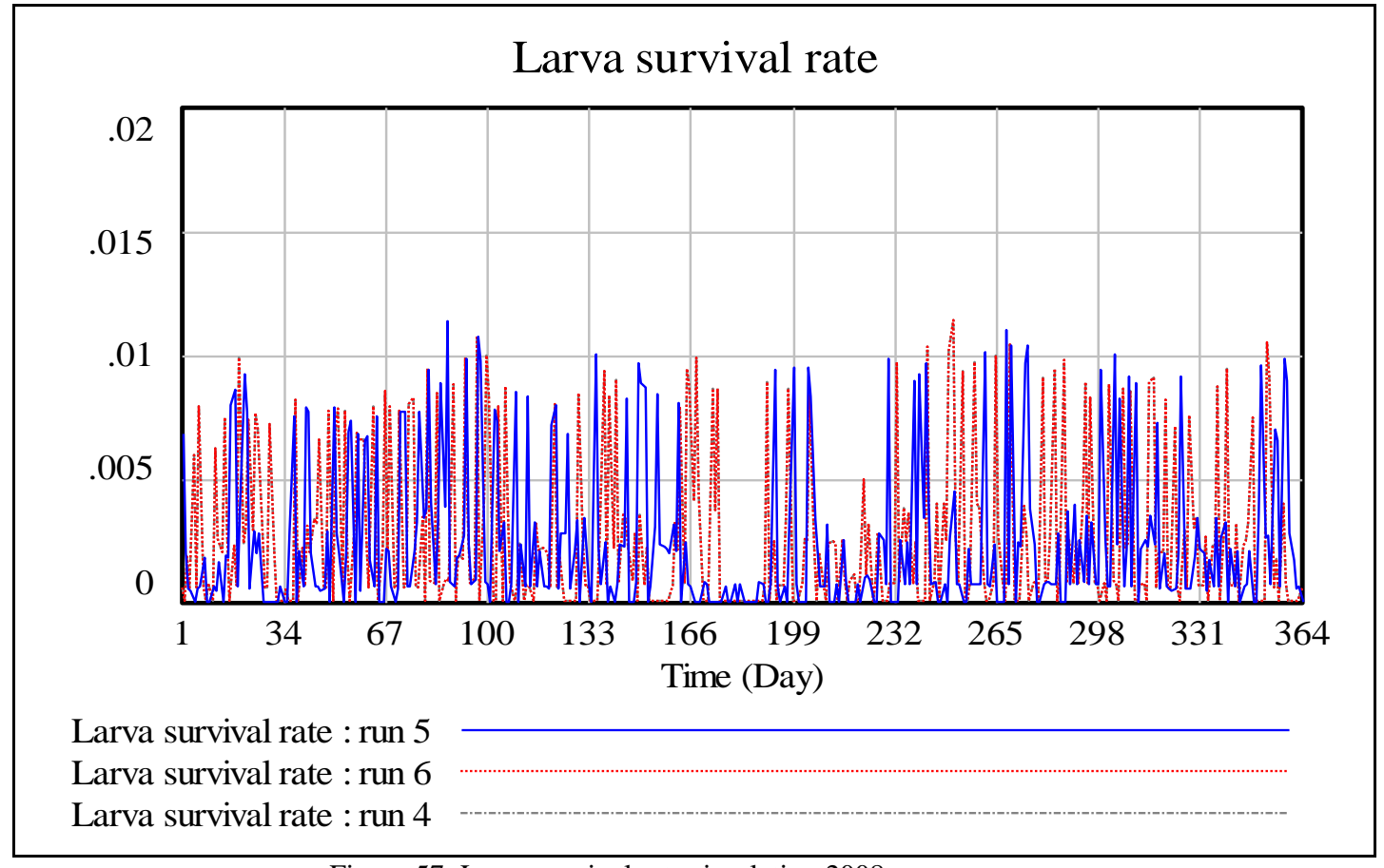

Figure 57: Larva survival rate simulation 2008 


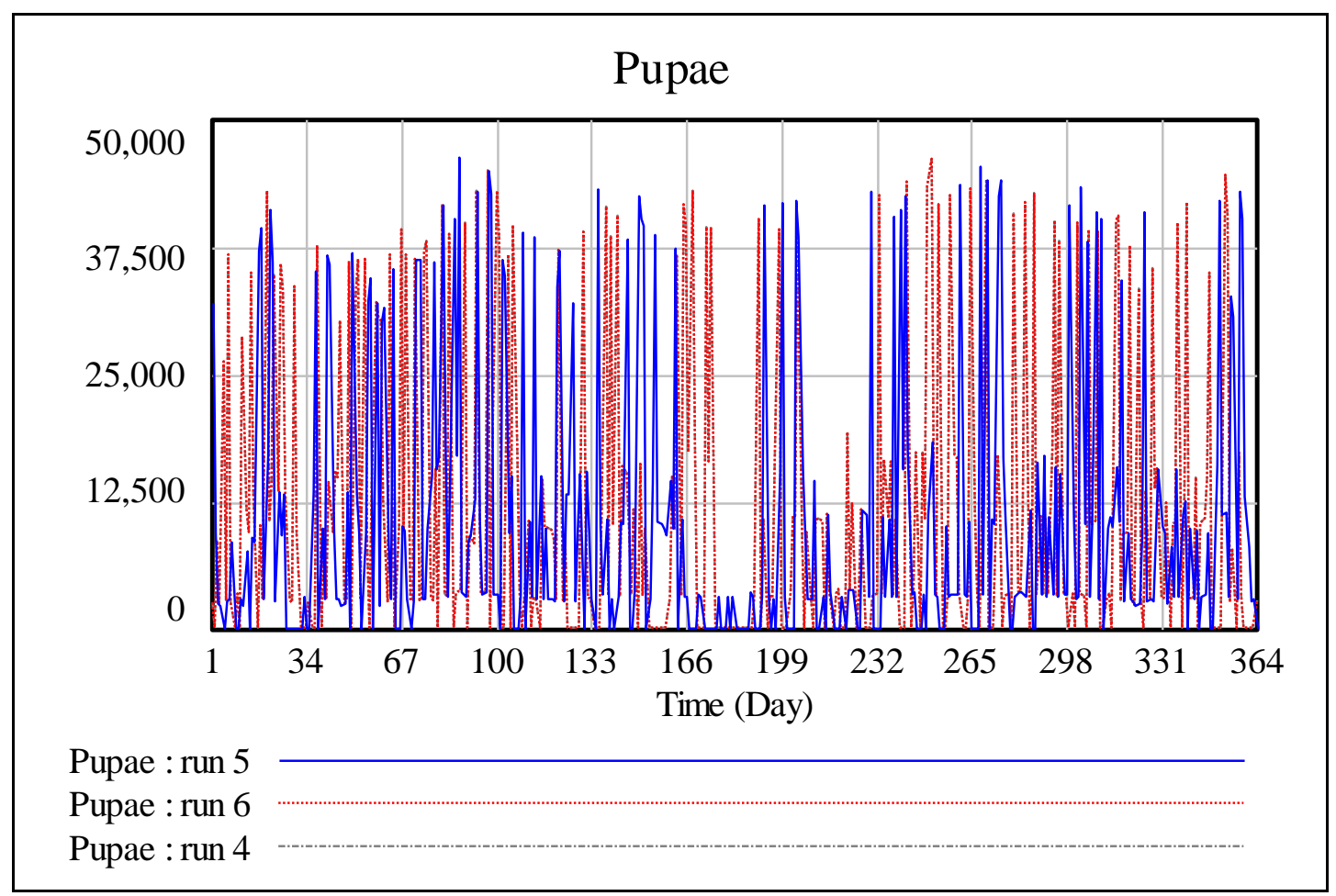

Figure 58: Pupae simulation 2008

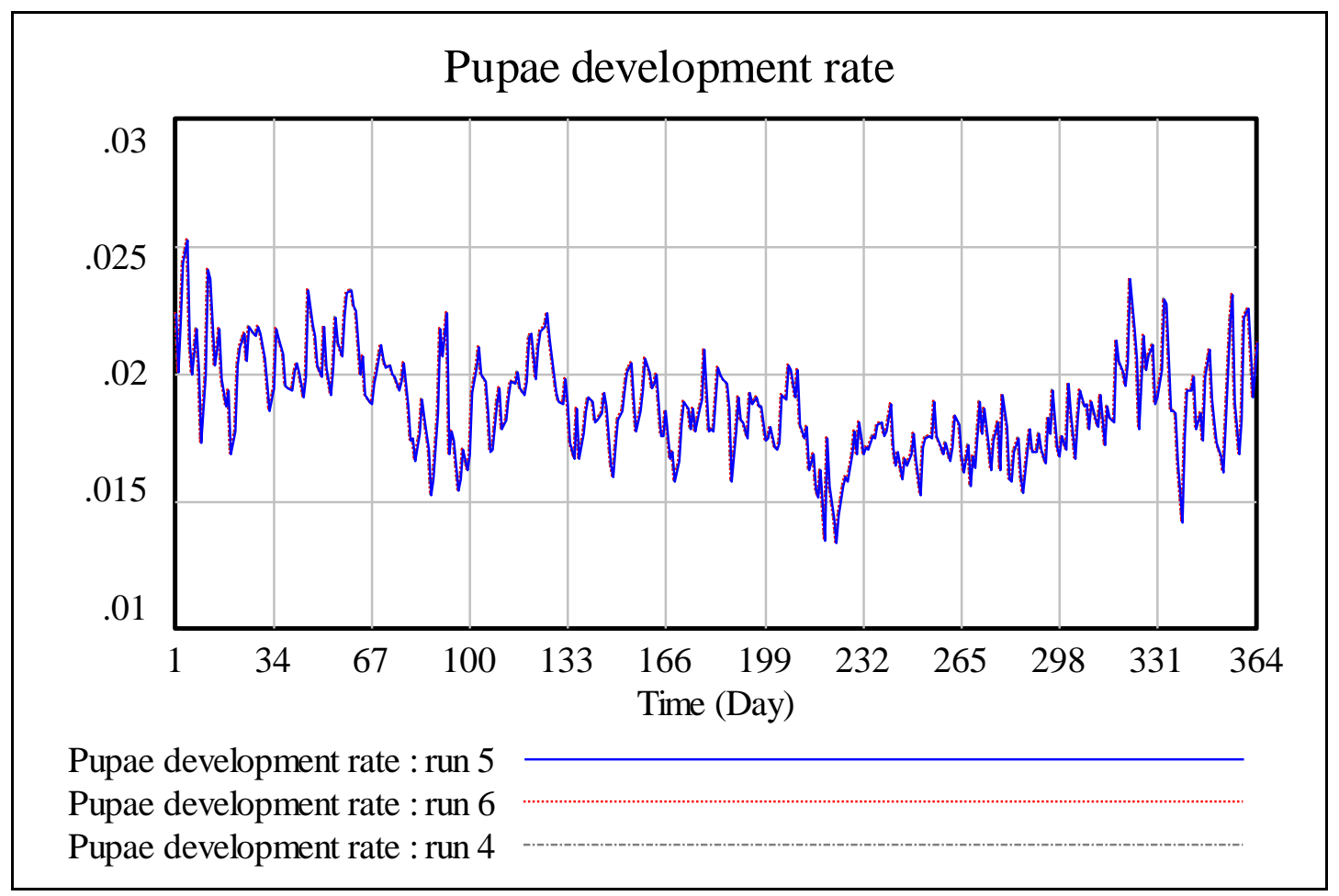

Figure 59: Pupae development rate simulation 2008 


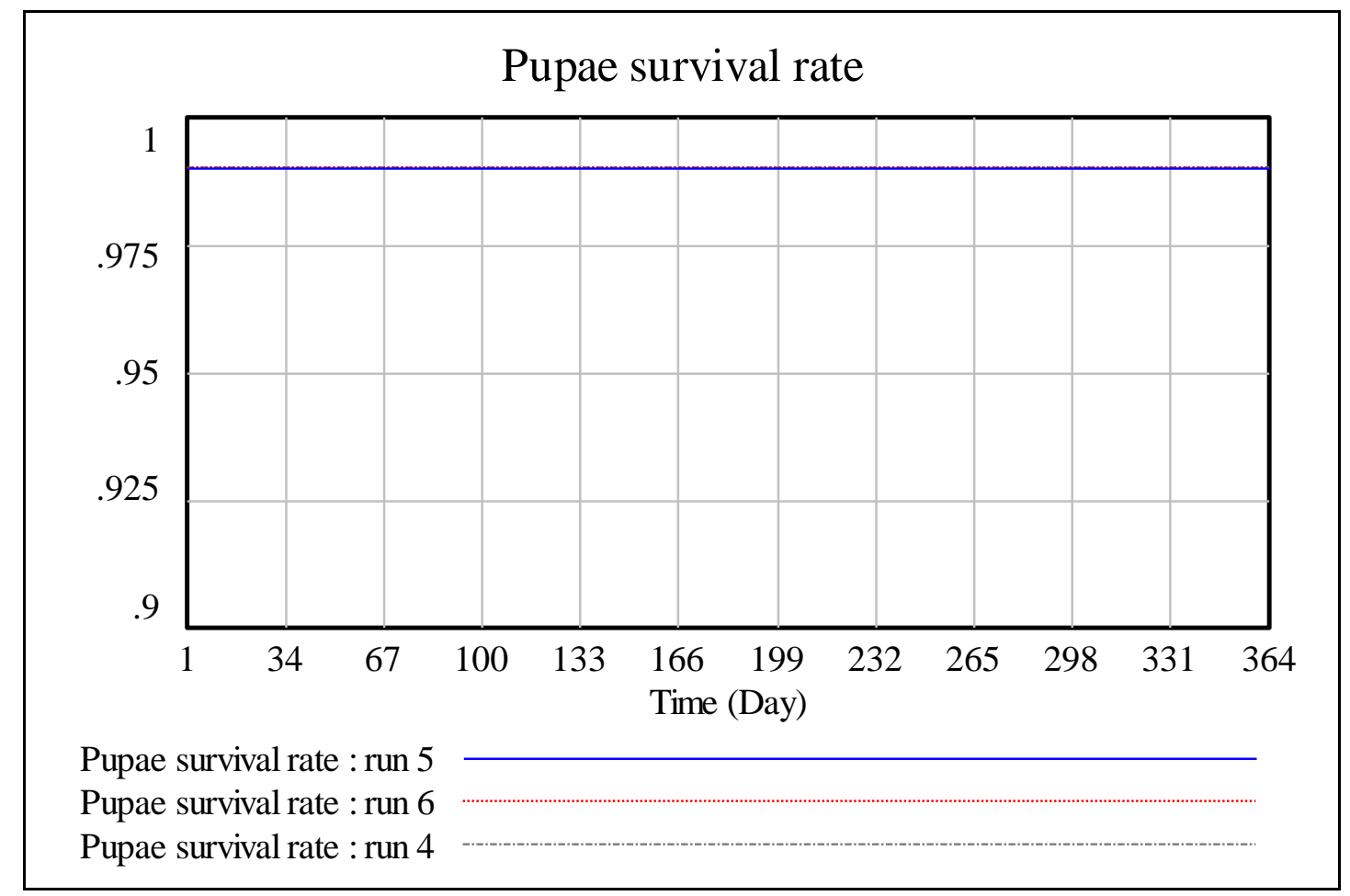

Figure 60: Pupae survival rate simulation 2008

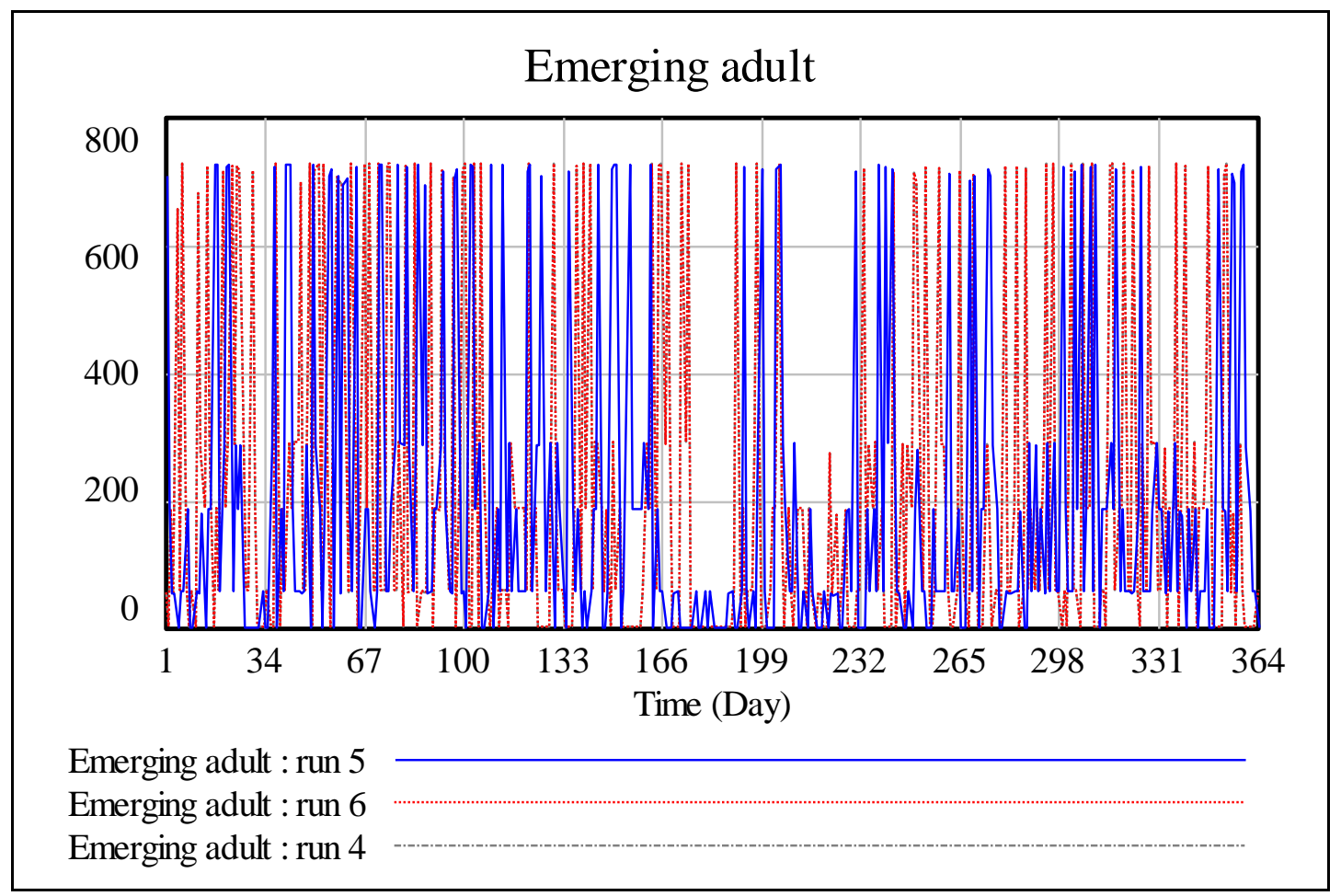

Figure 61: Emerging adult simulation 2008 


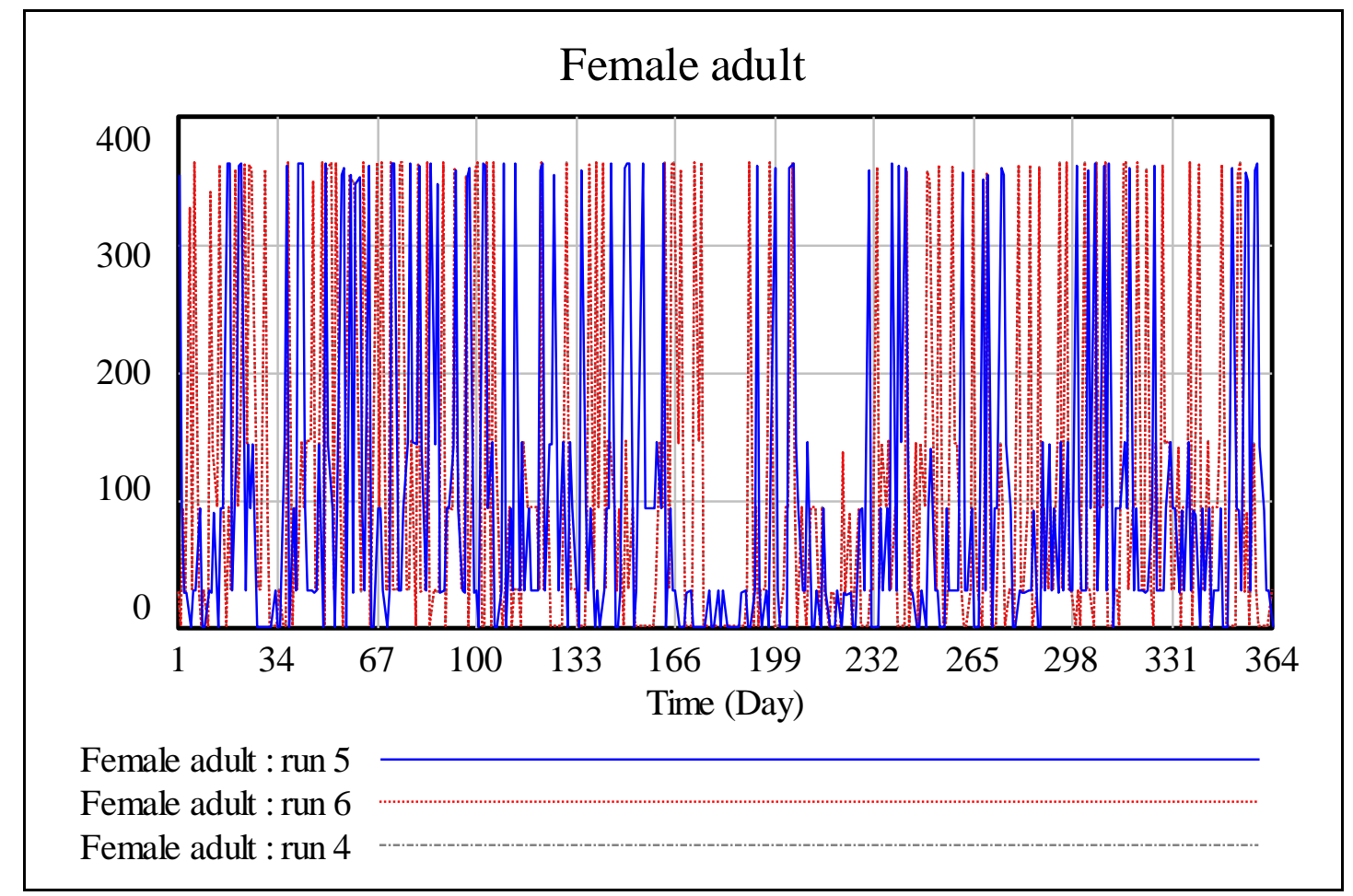

Figure 62: Female adult simulation 2008

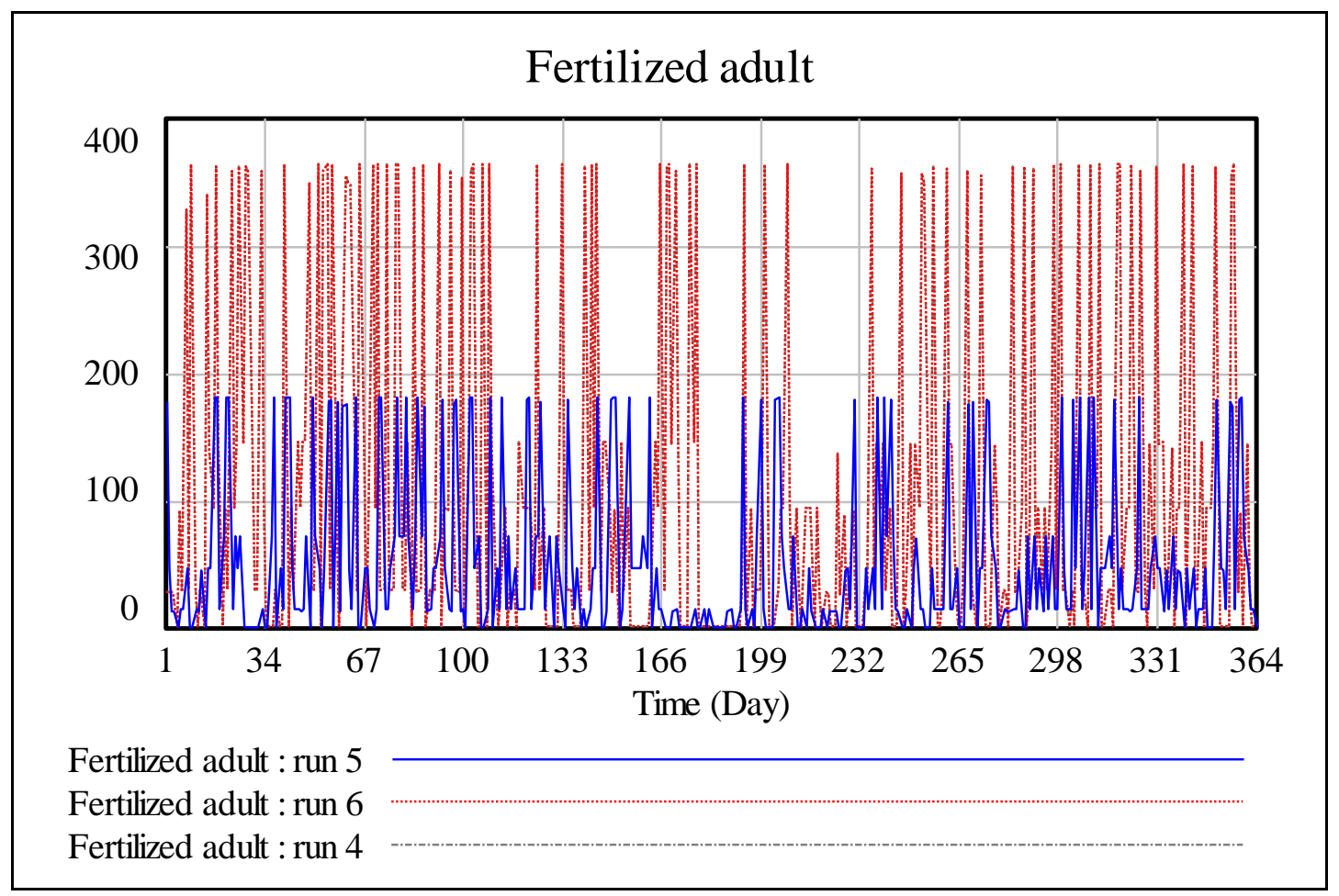

Figure 63: Fertilized adult simulation 2008 


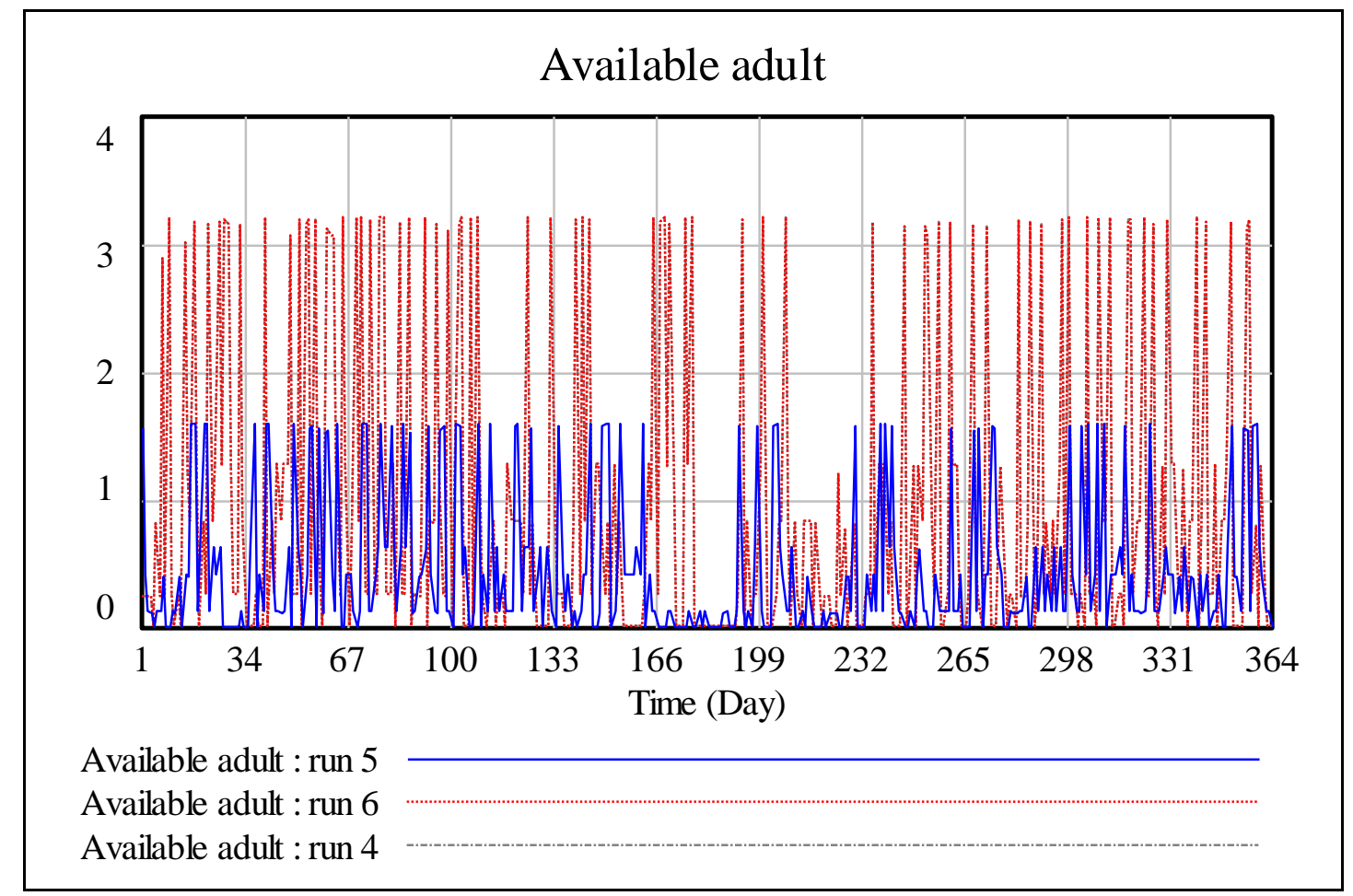

Figure 64: Available adult simulation 2008

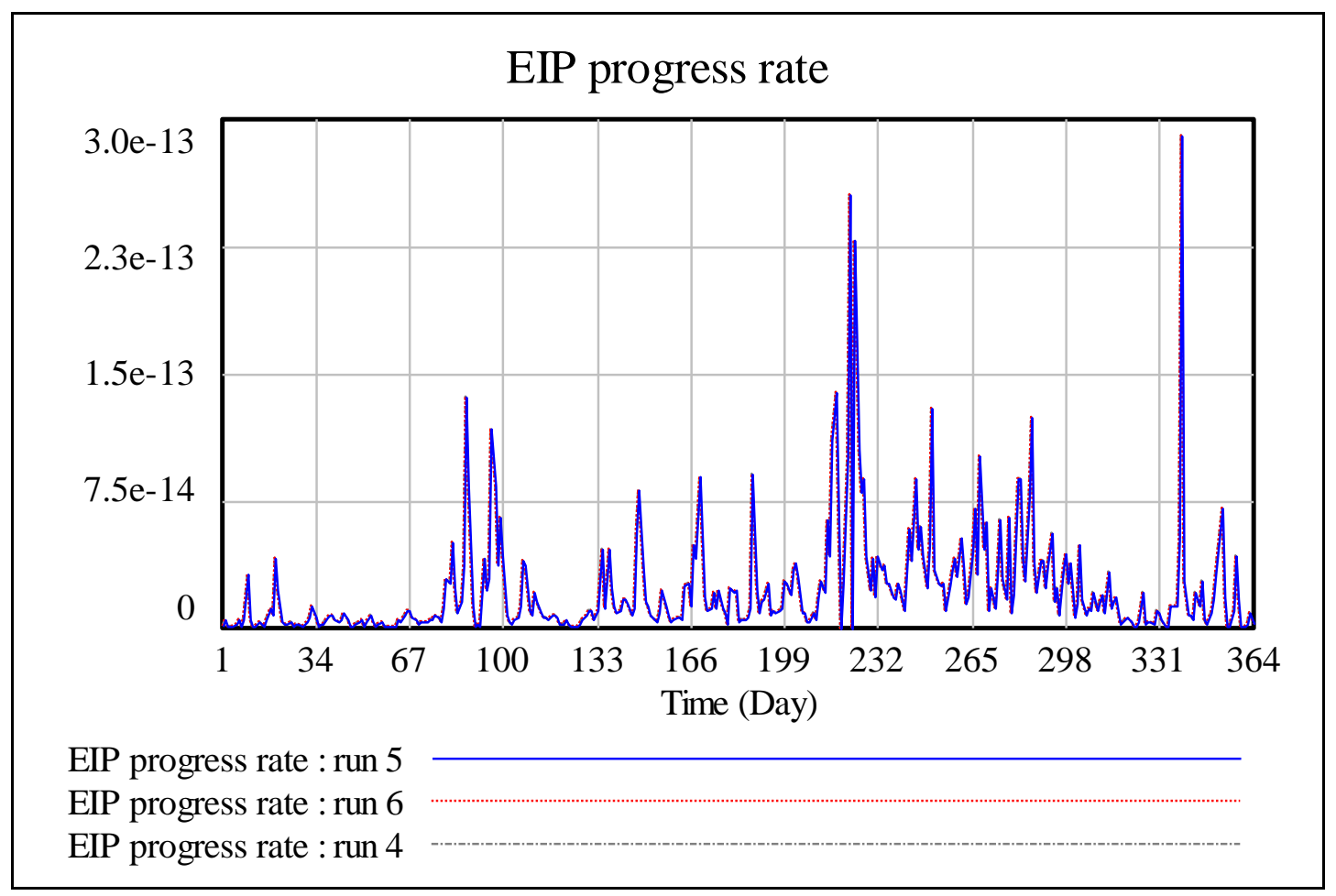

Figure 65: EIP progress rate simulation 2008 


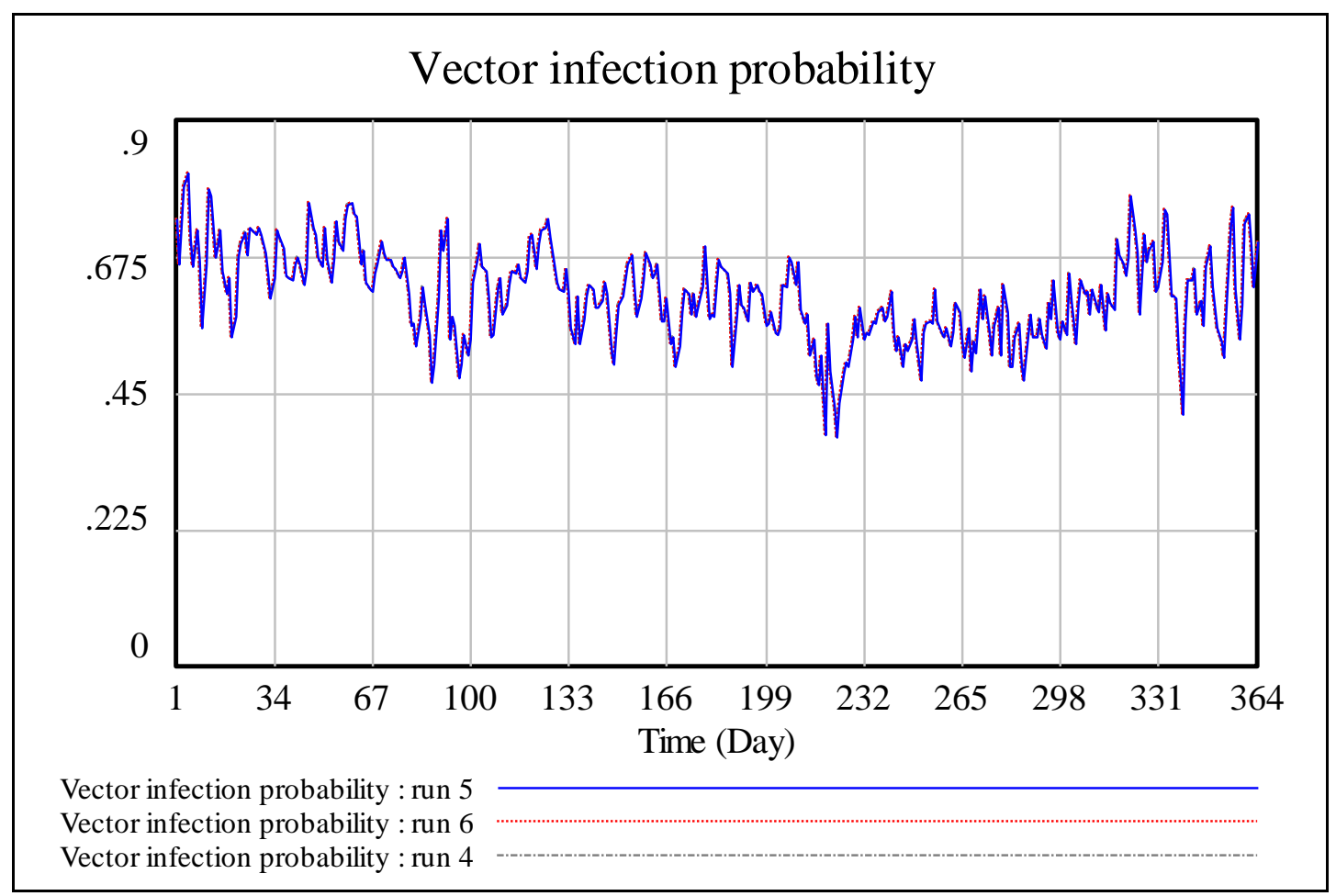

Figure 66: Vector infection probability simulation 2008

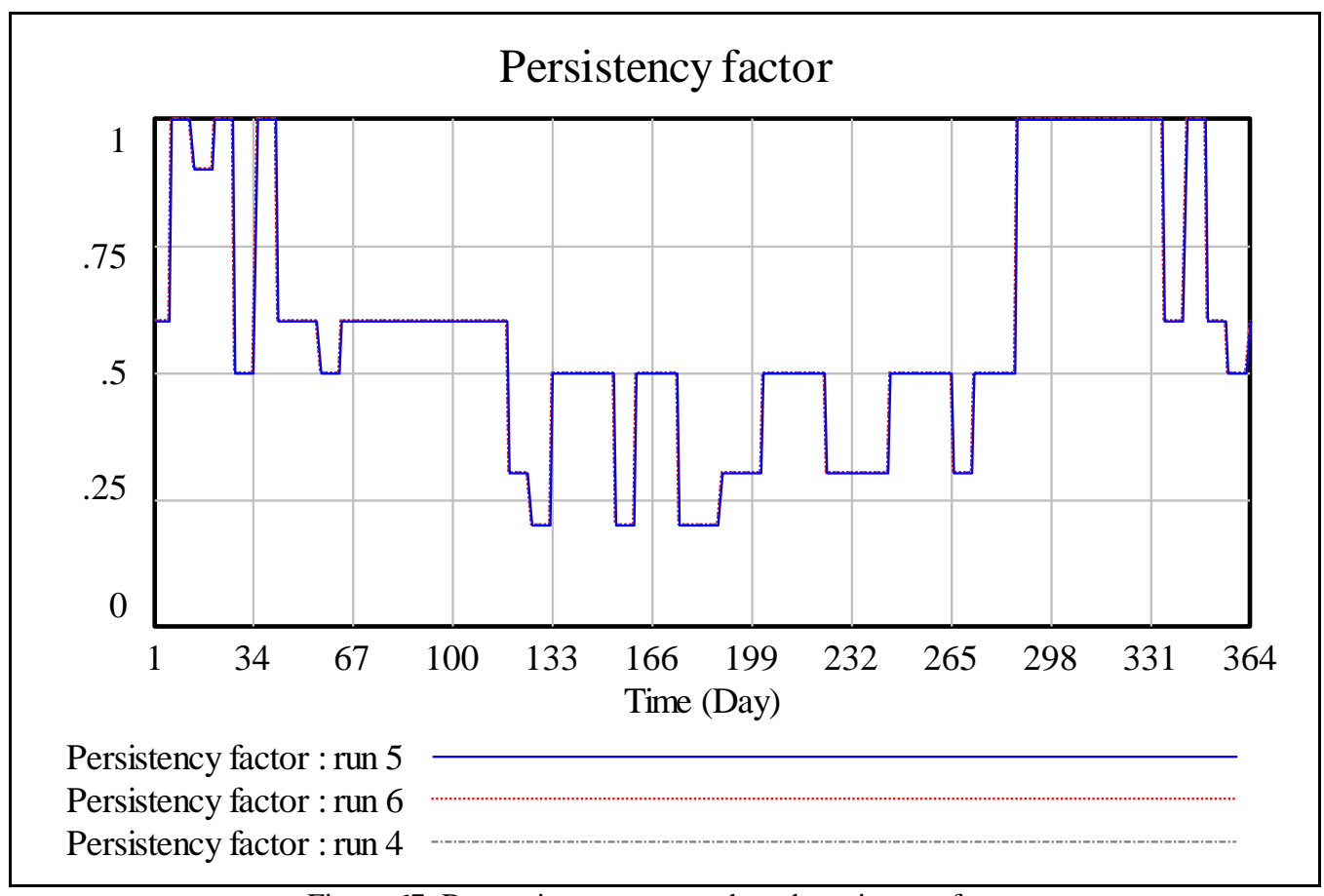

Figure 67: Dew point temperature based persistency factor simulation 2008 


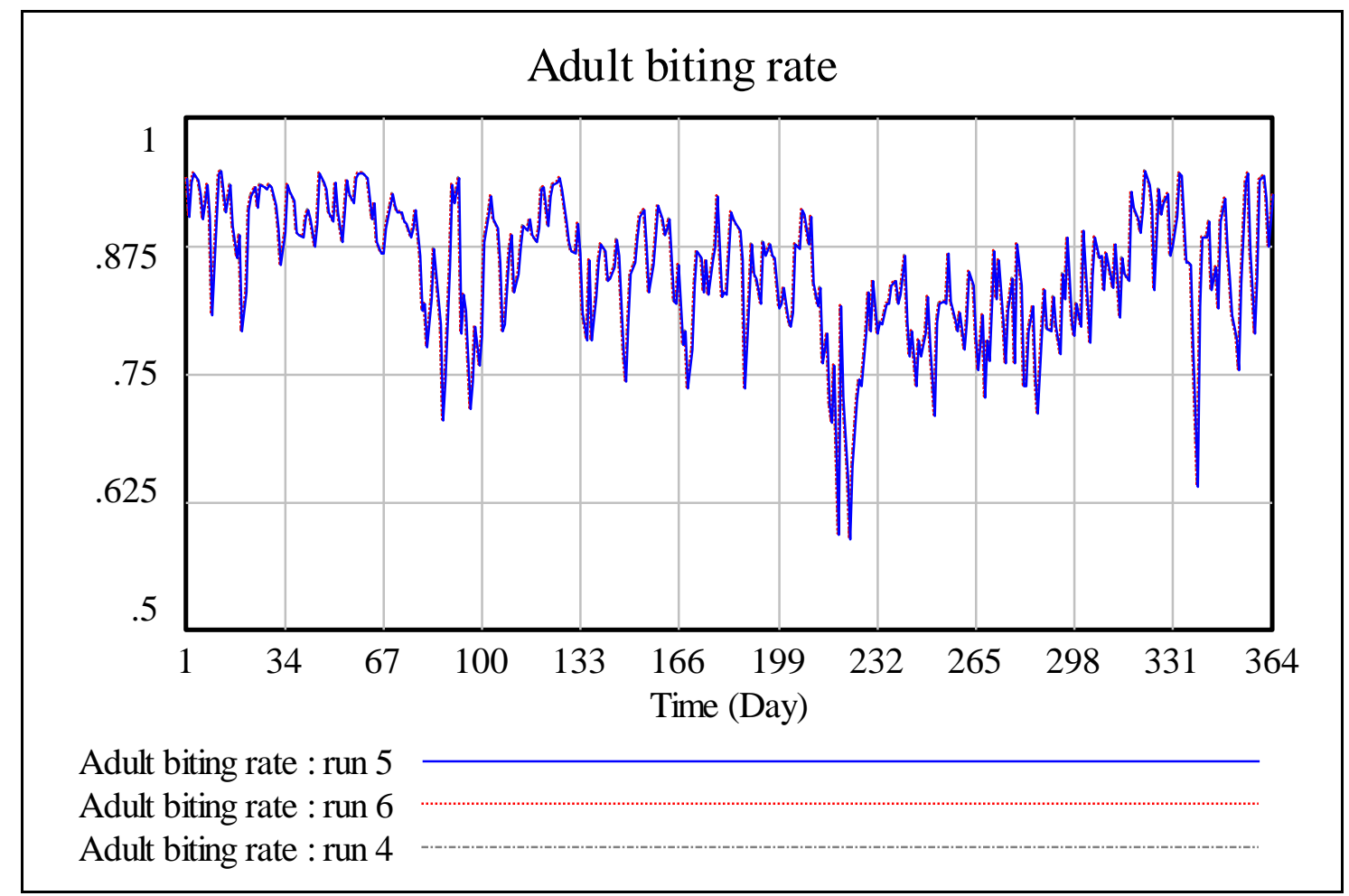

Figure 68: Adult biting rate simulation 2008

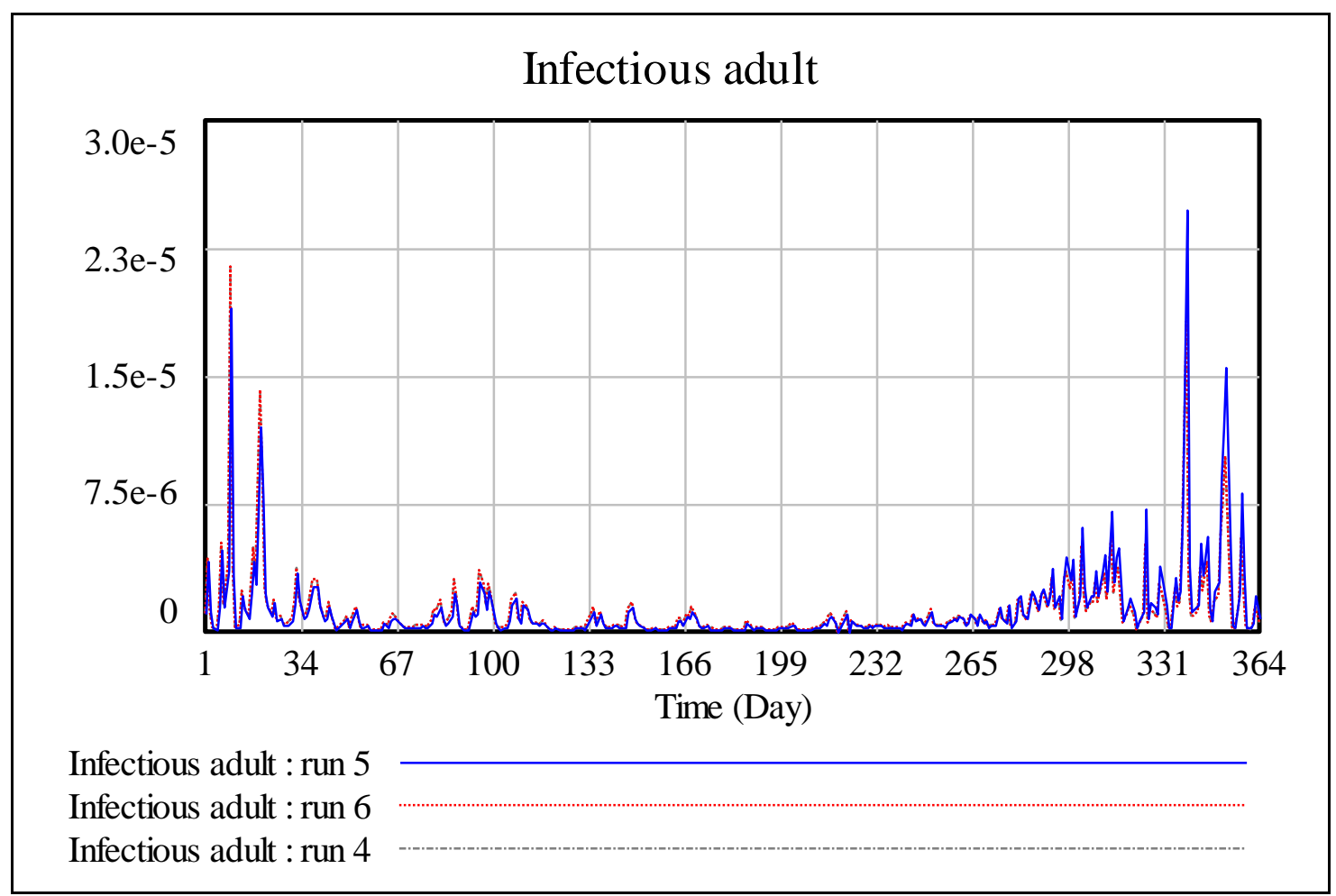

Figure 69: Infectious adult simulation 2008 


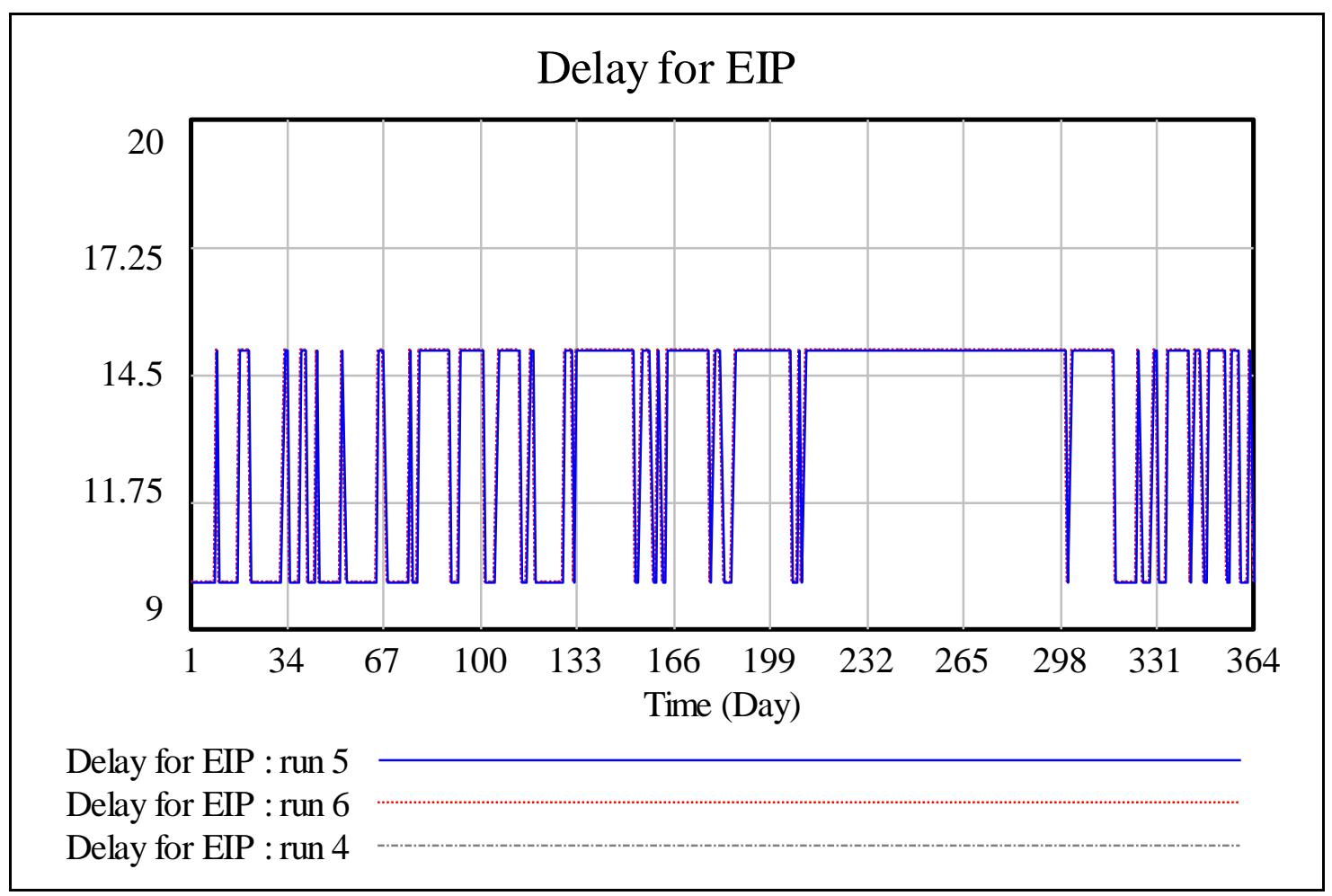

Figure 70: Delay for EIP simulation 2008

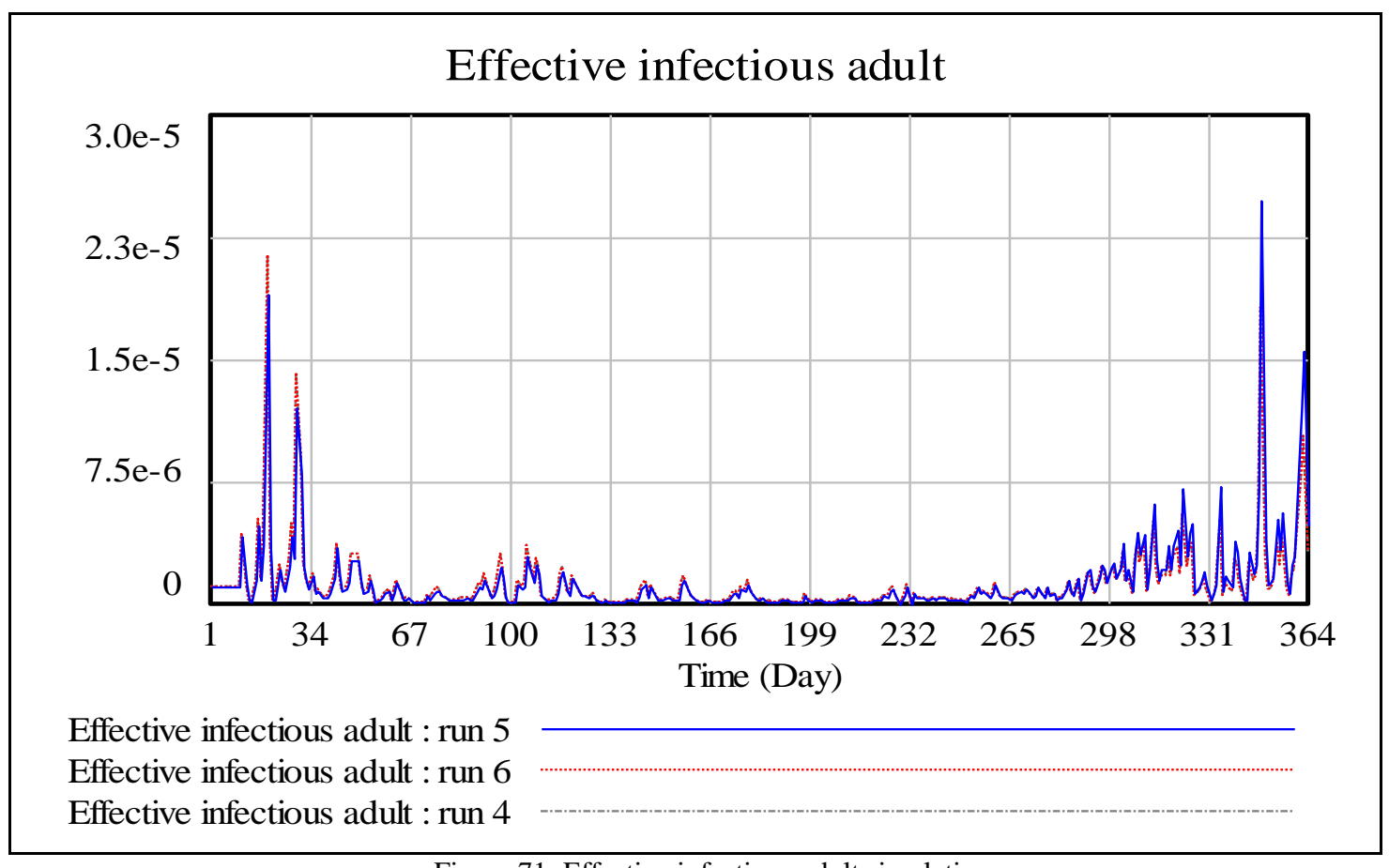

Figure 71: Effective infectious adult simulation 


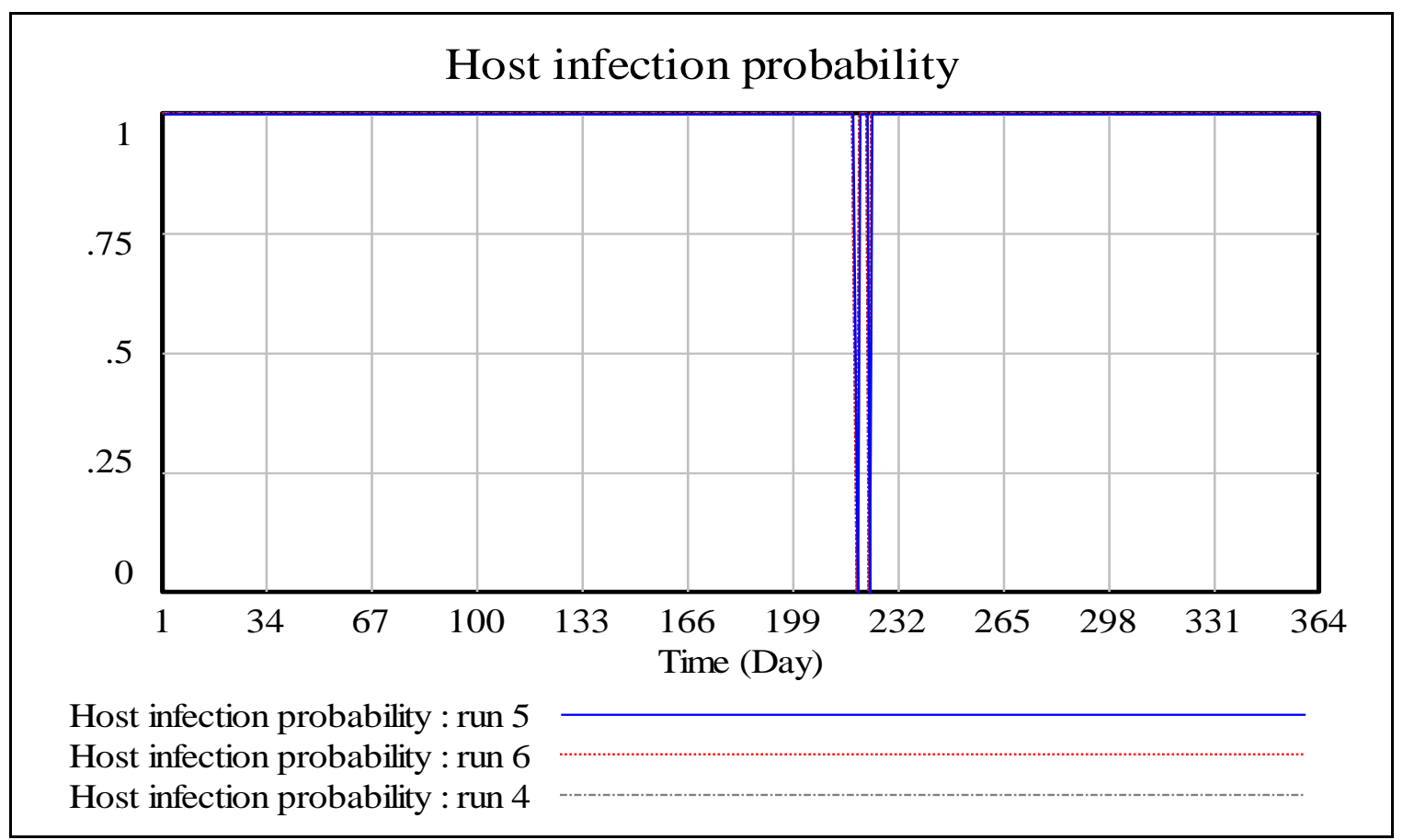

Figure 72: Host infection probability simulation

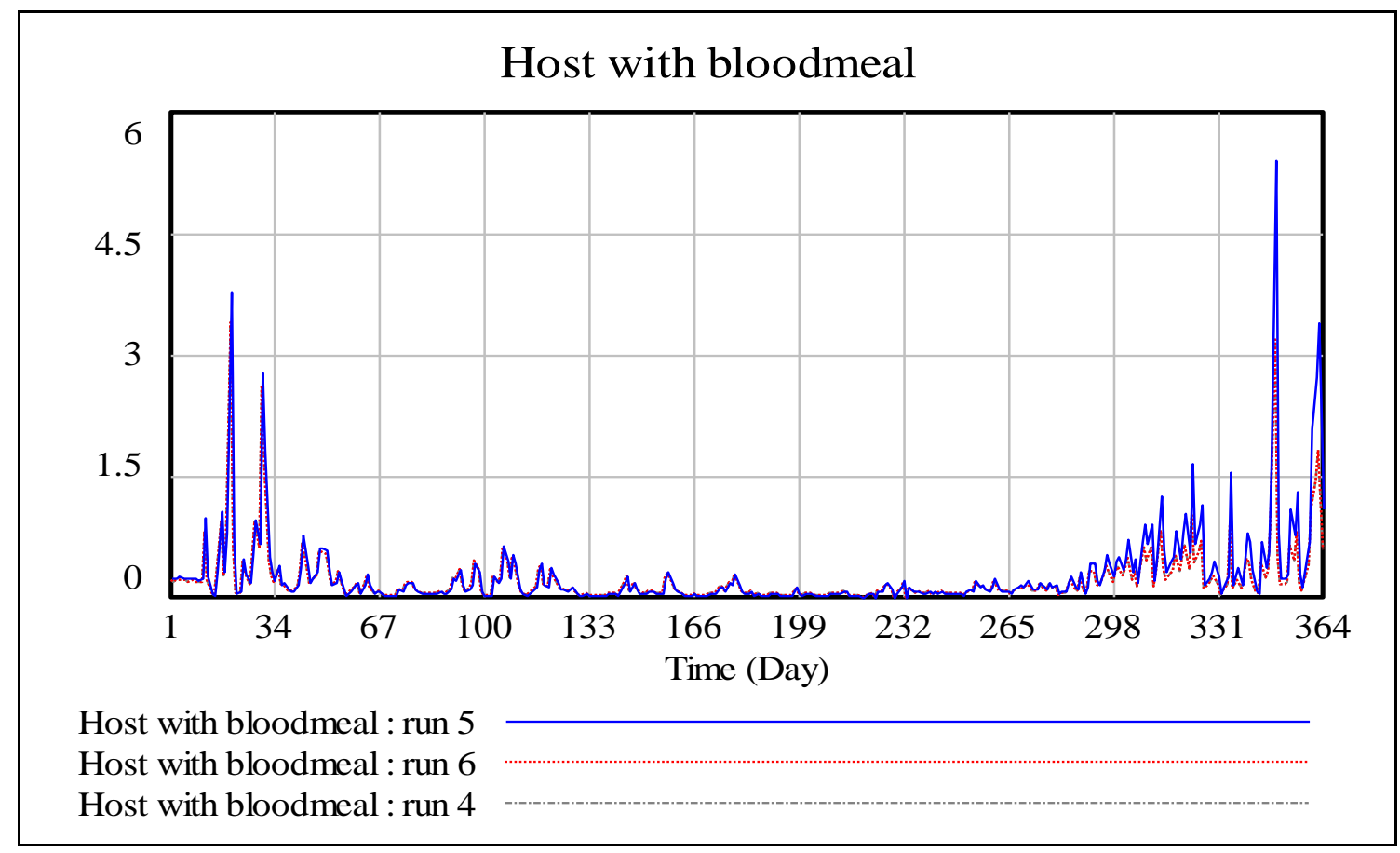

Figure 73: Host with bloodmeal simulation 2008 


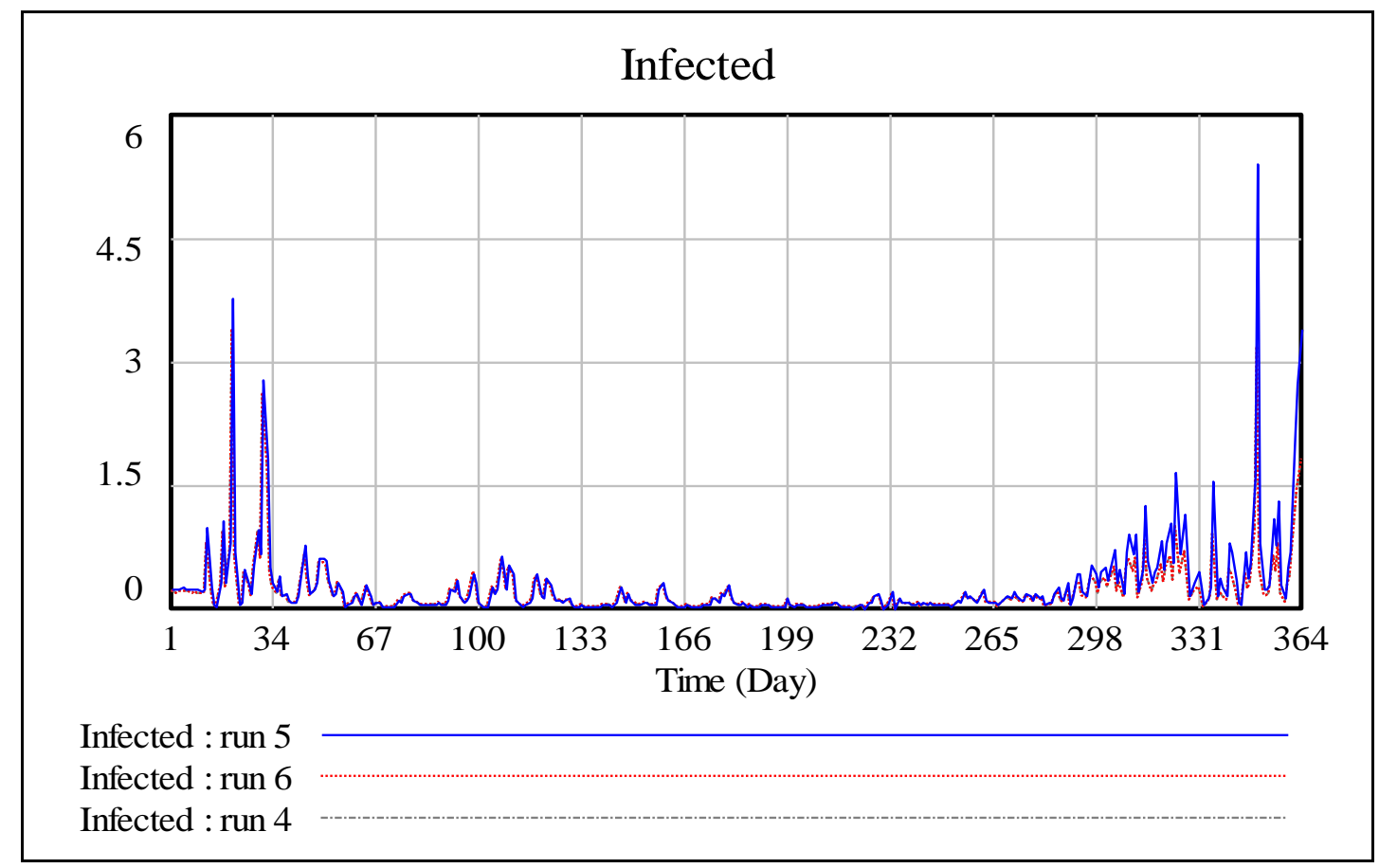

Figure 74: Infected simulation 2008

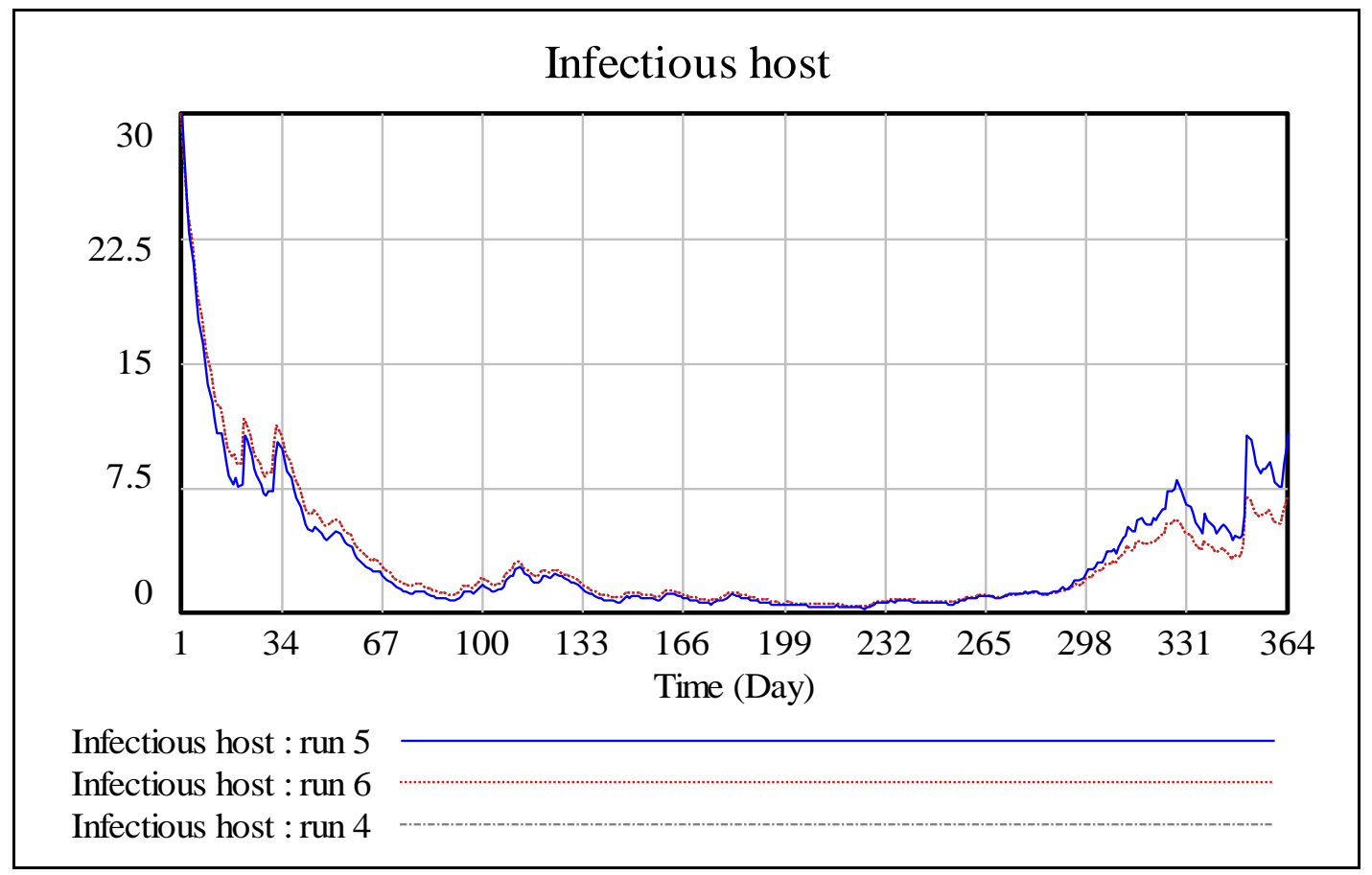

Figure 75: Infectious host simulation 2008 
Vensim simulations 2004

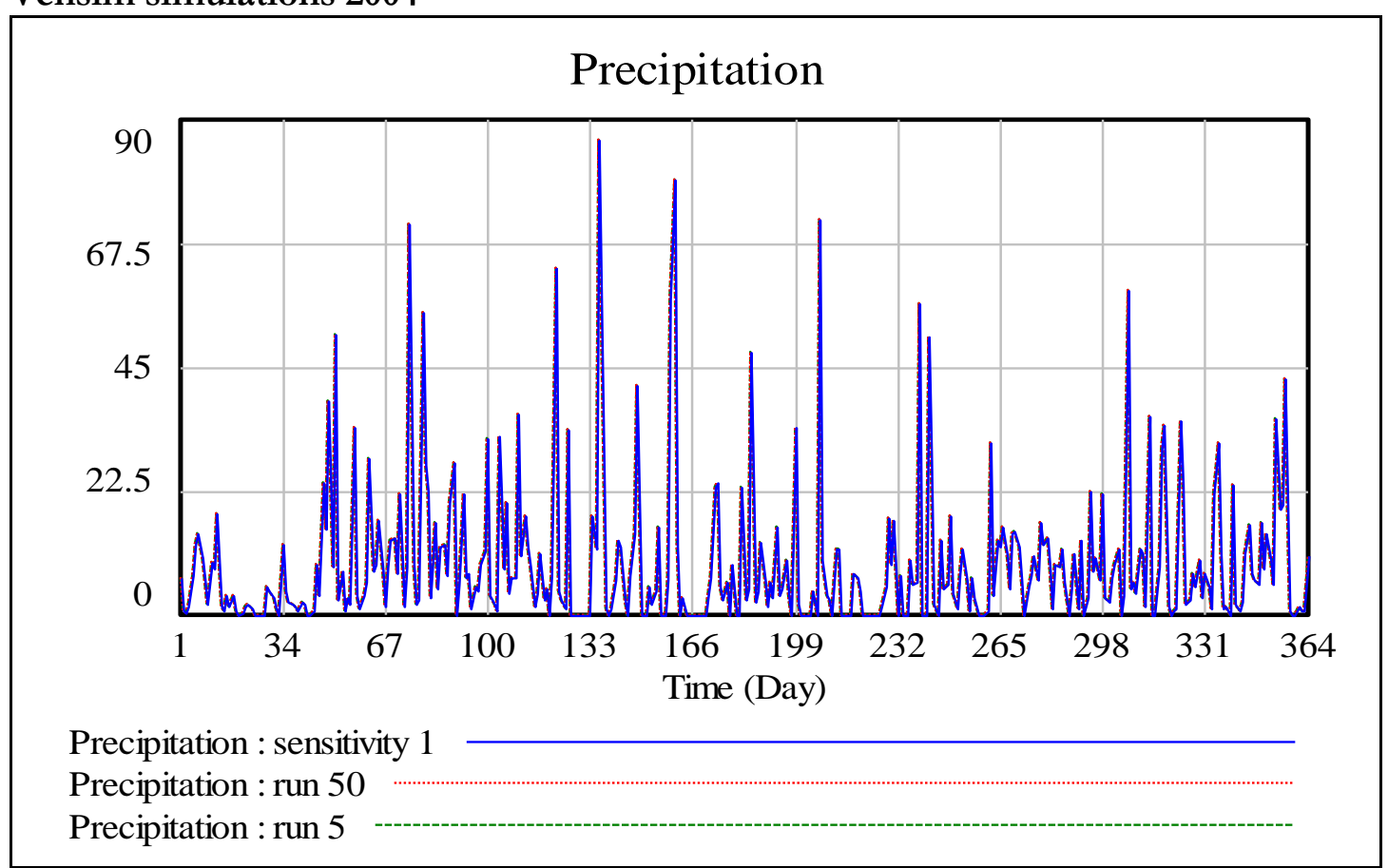

Figure 76: Precipitation simulation 2004

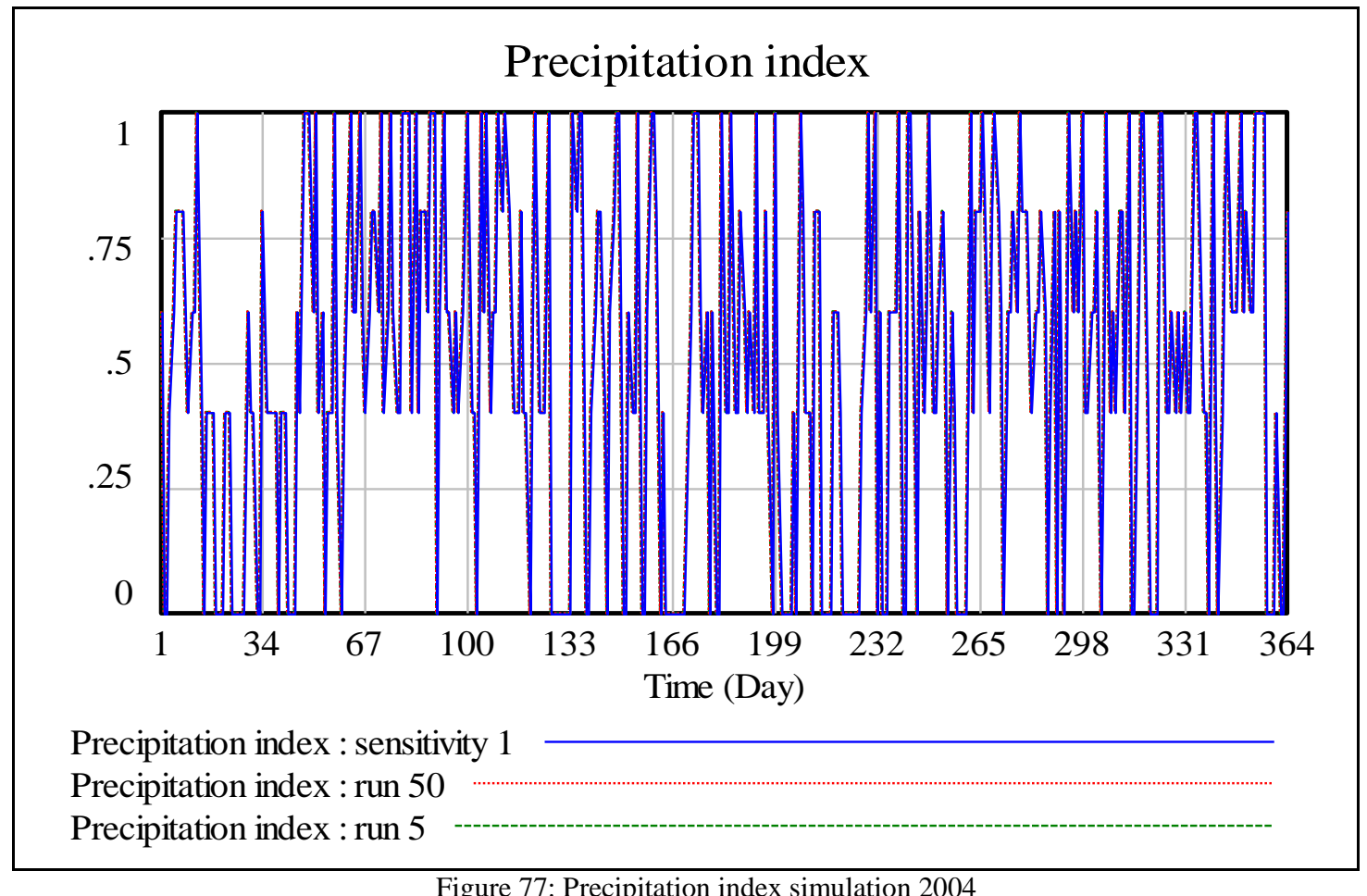

Figure 77: Precipitation index simulation 2004 


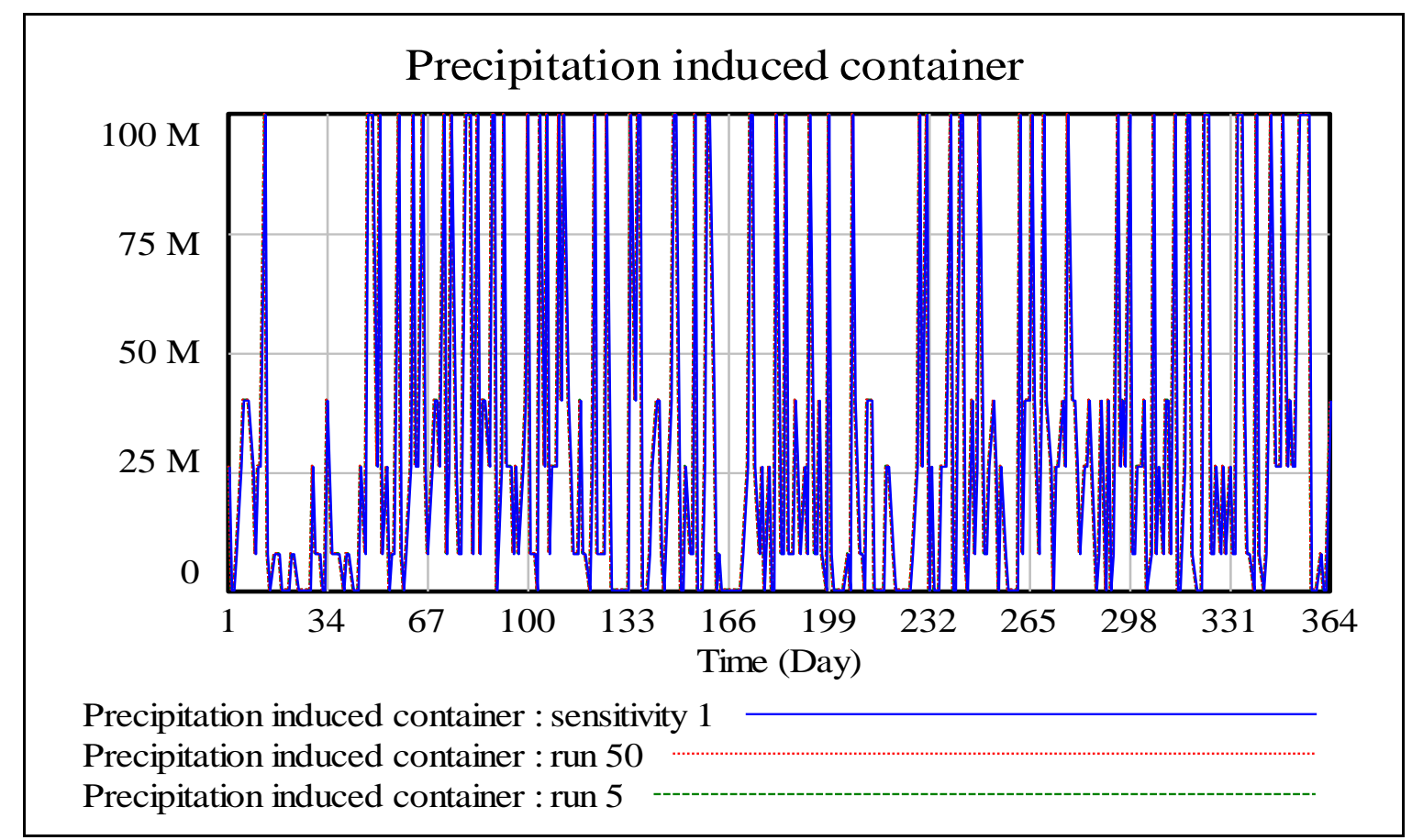

Figure 78: Precipitation induced container simulation 2004

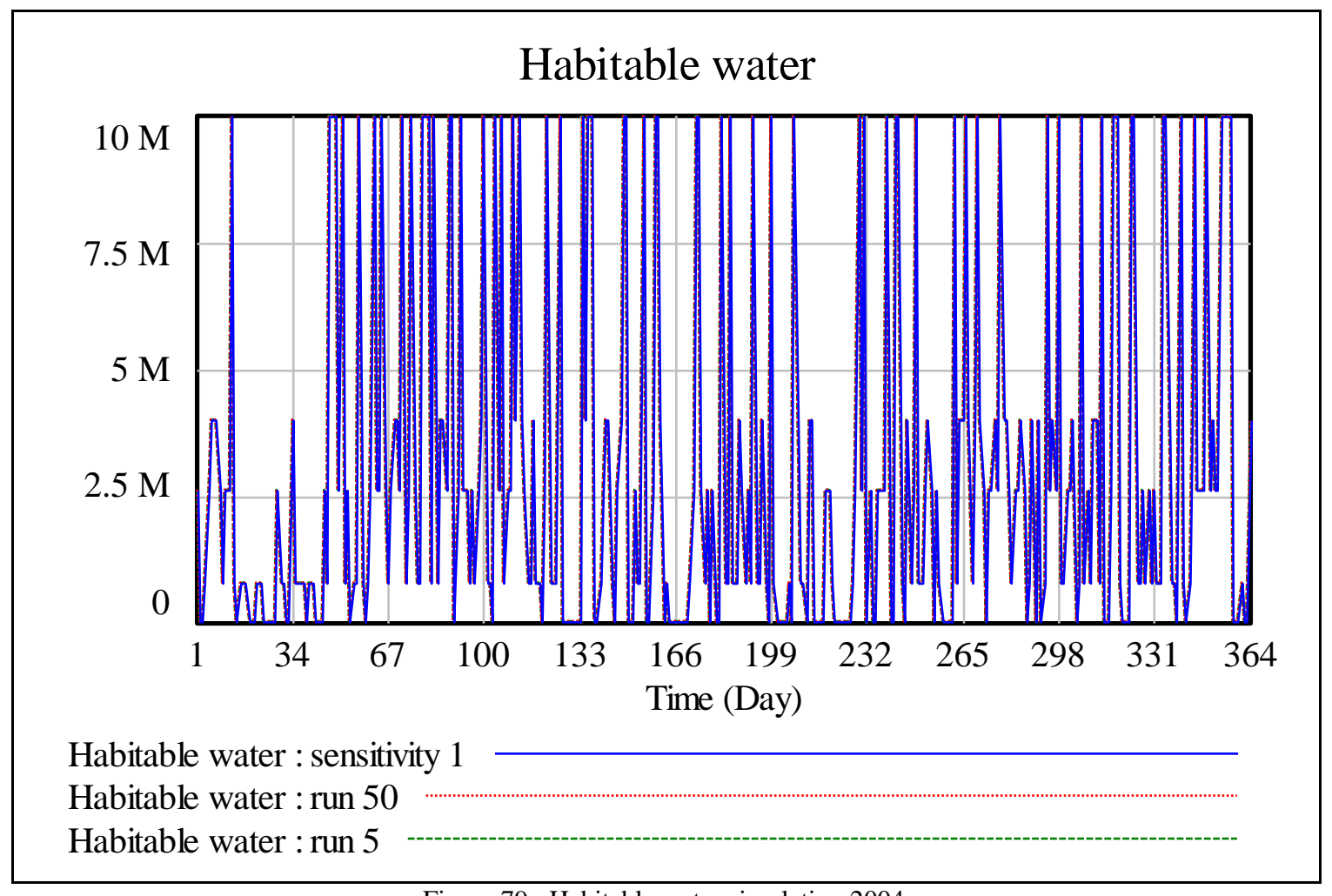

Figure 79 : Habitable water simulation 2004 


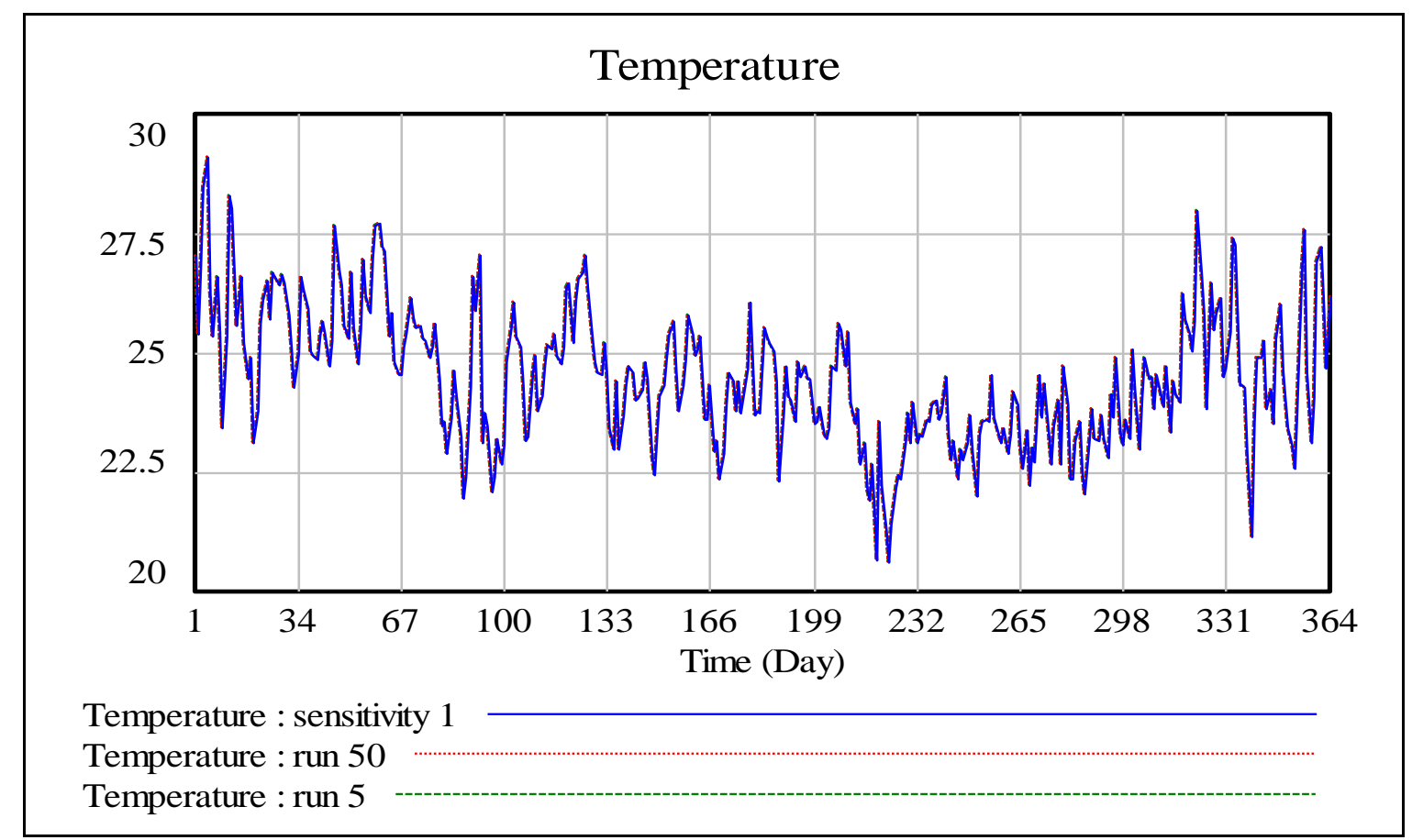

Figure 80: Temperature simulation 2004

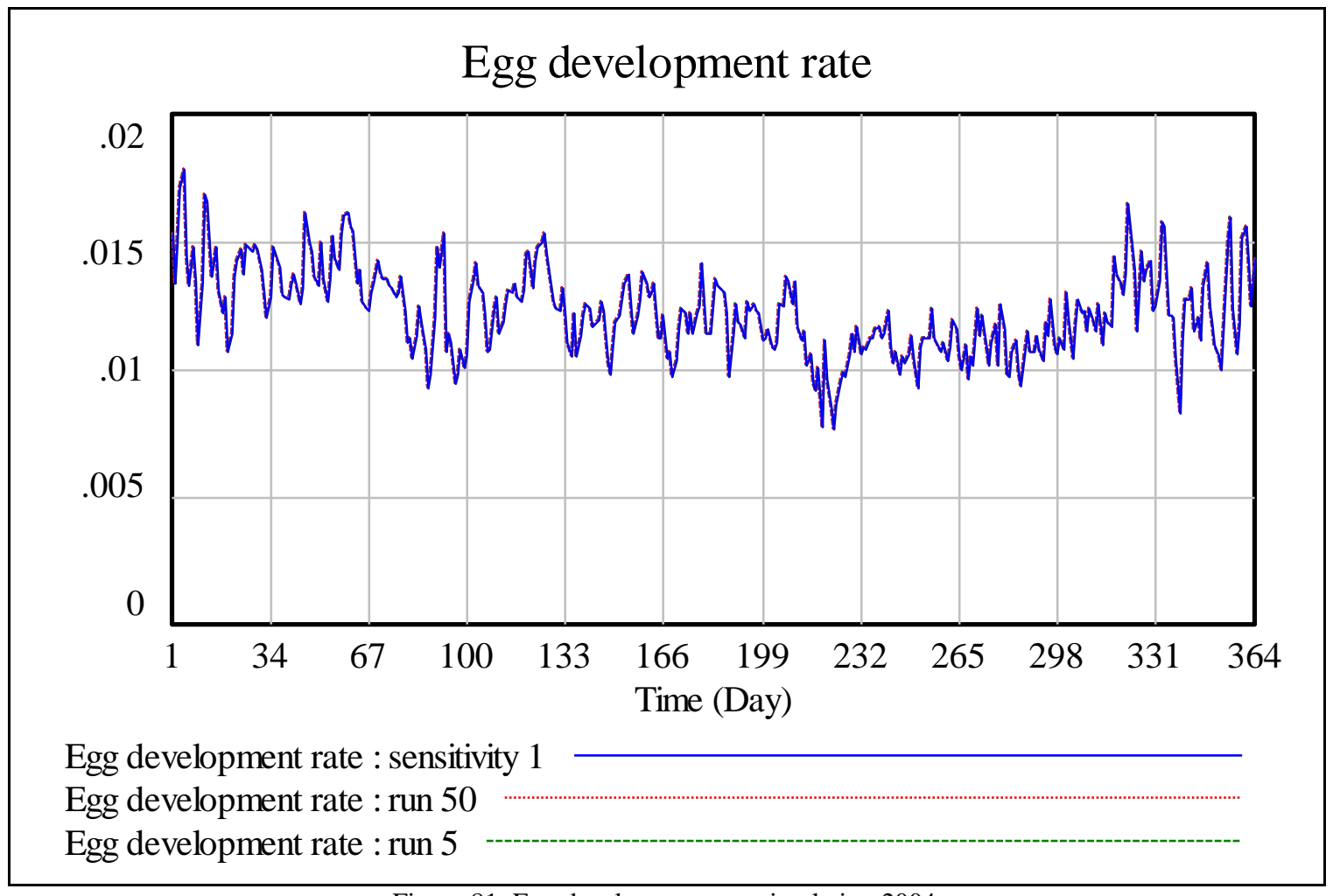

Figure 81: Egg development rate simulation 2004 


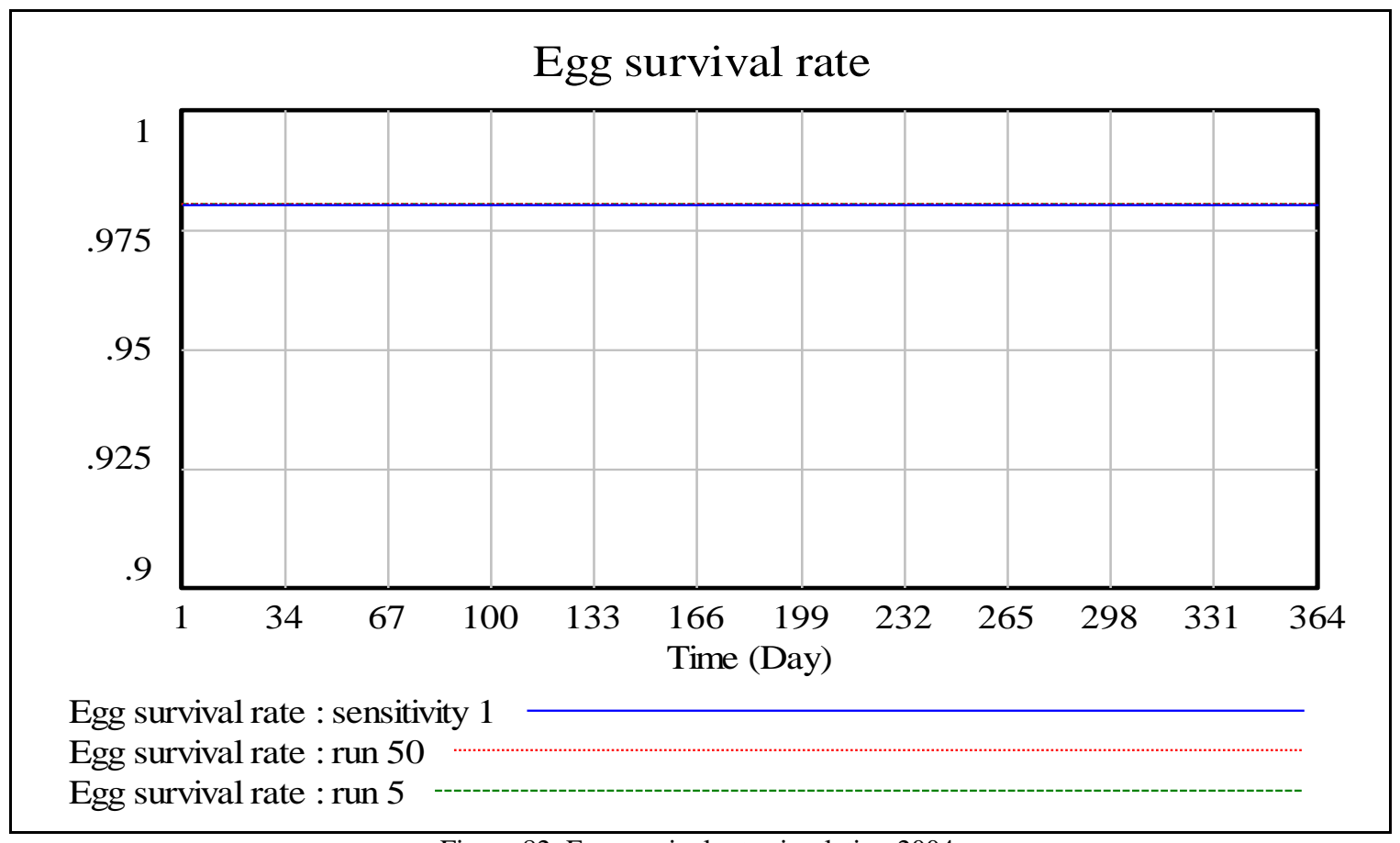

Figure 82: Egg survival rate simulation 2004

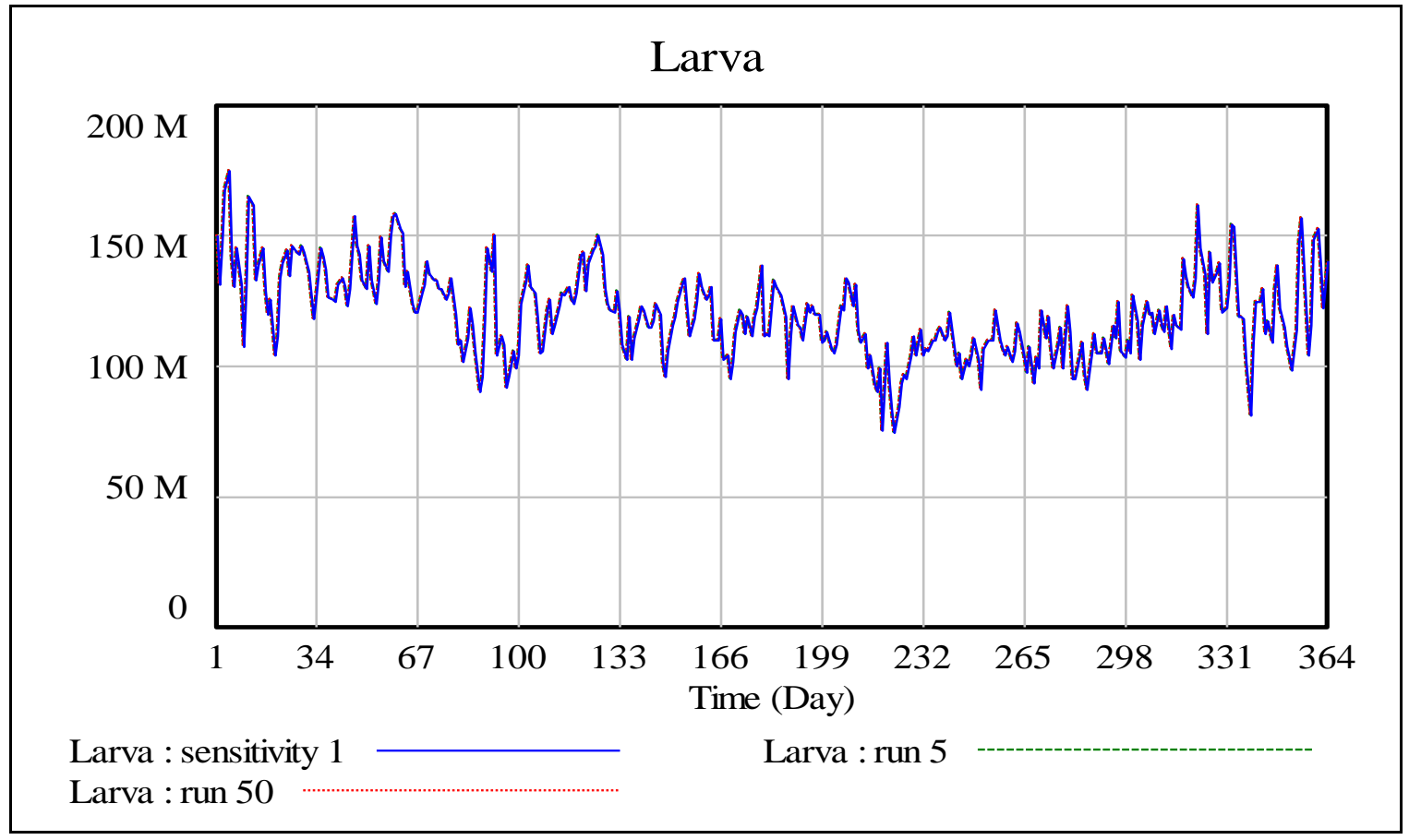

Figure 83: Larva simulation 2004 


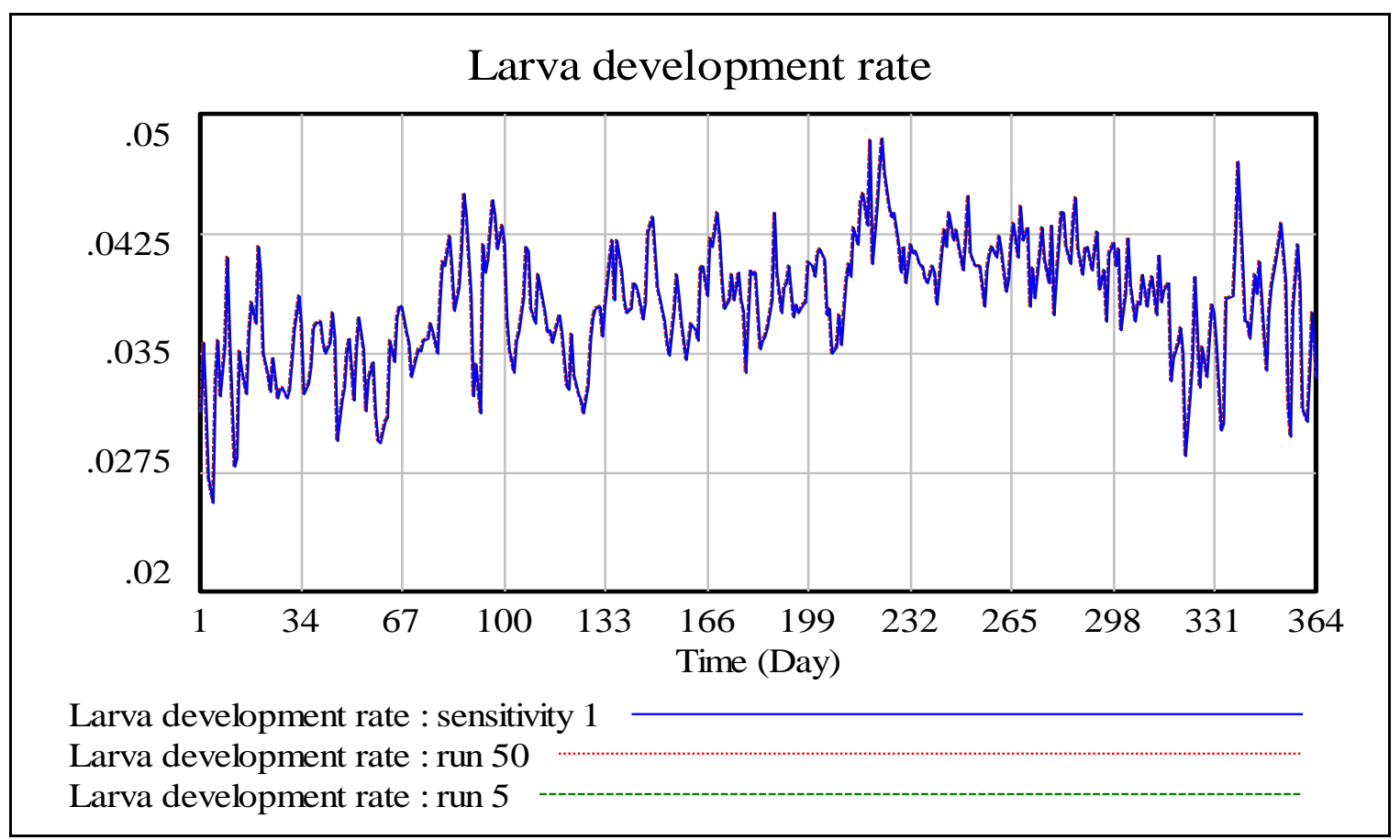

Figure 84: Larva development rate simulation 2004

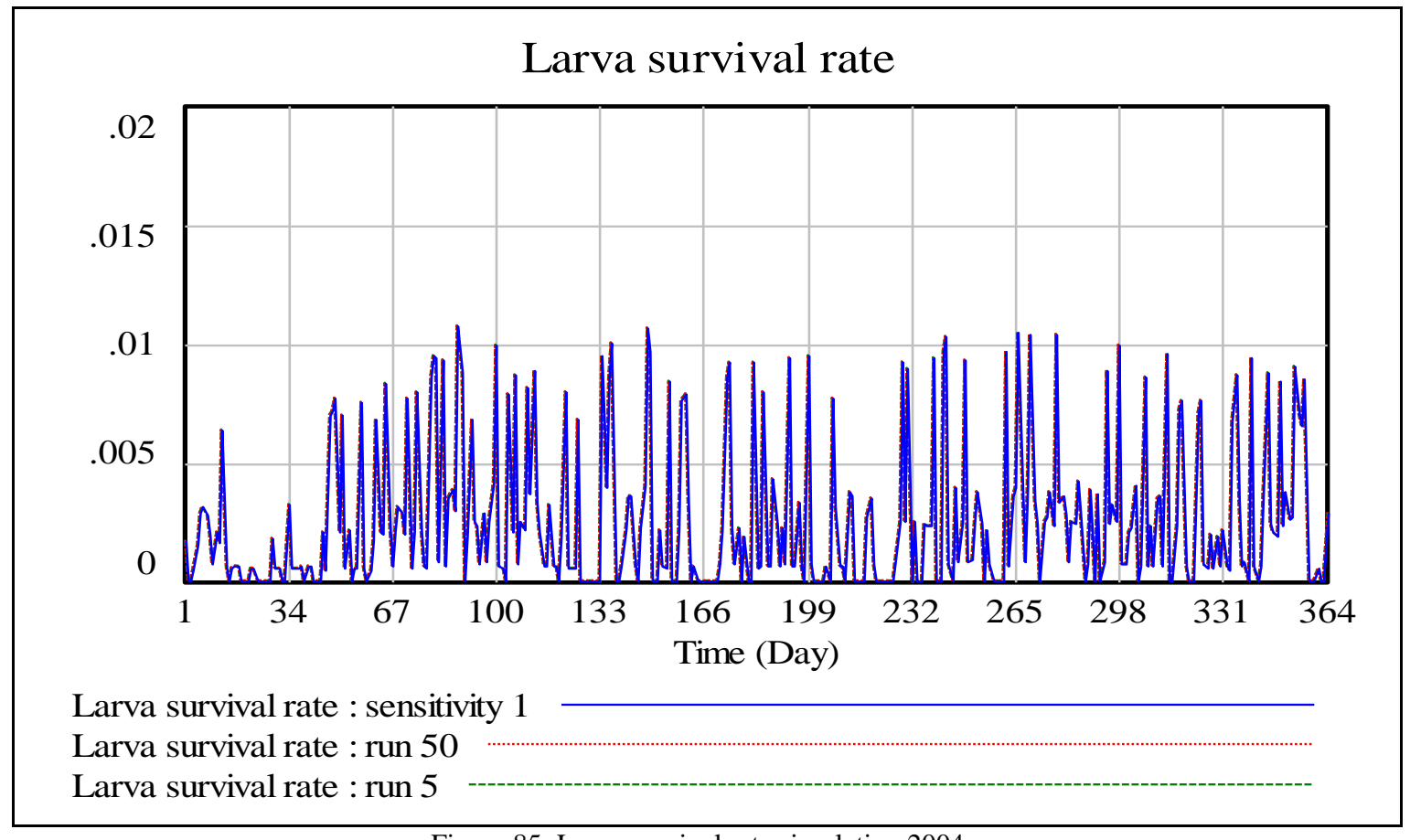

Figure 85: Larva survival rate simulation 2004 


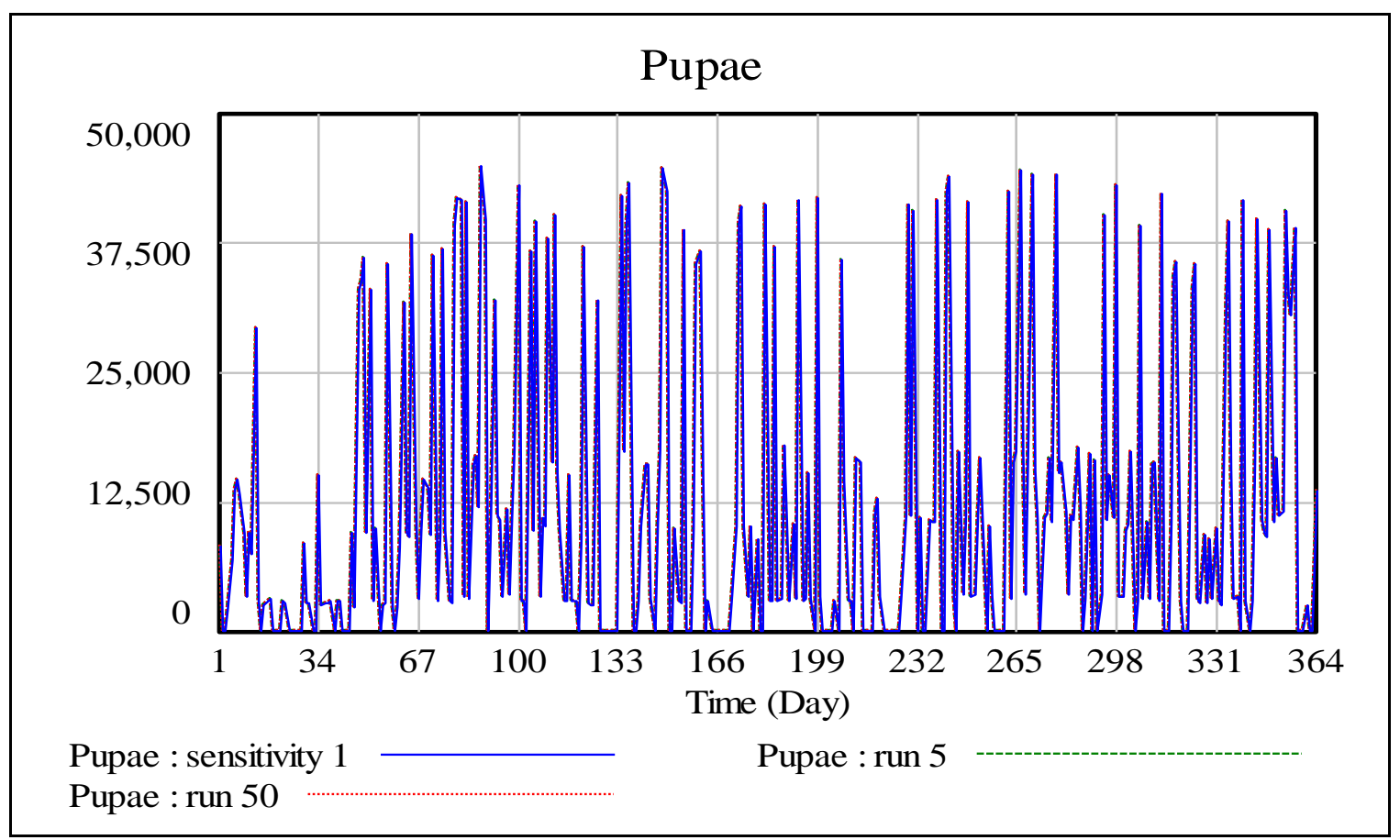

Figure 86: Pupae simulation 2004

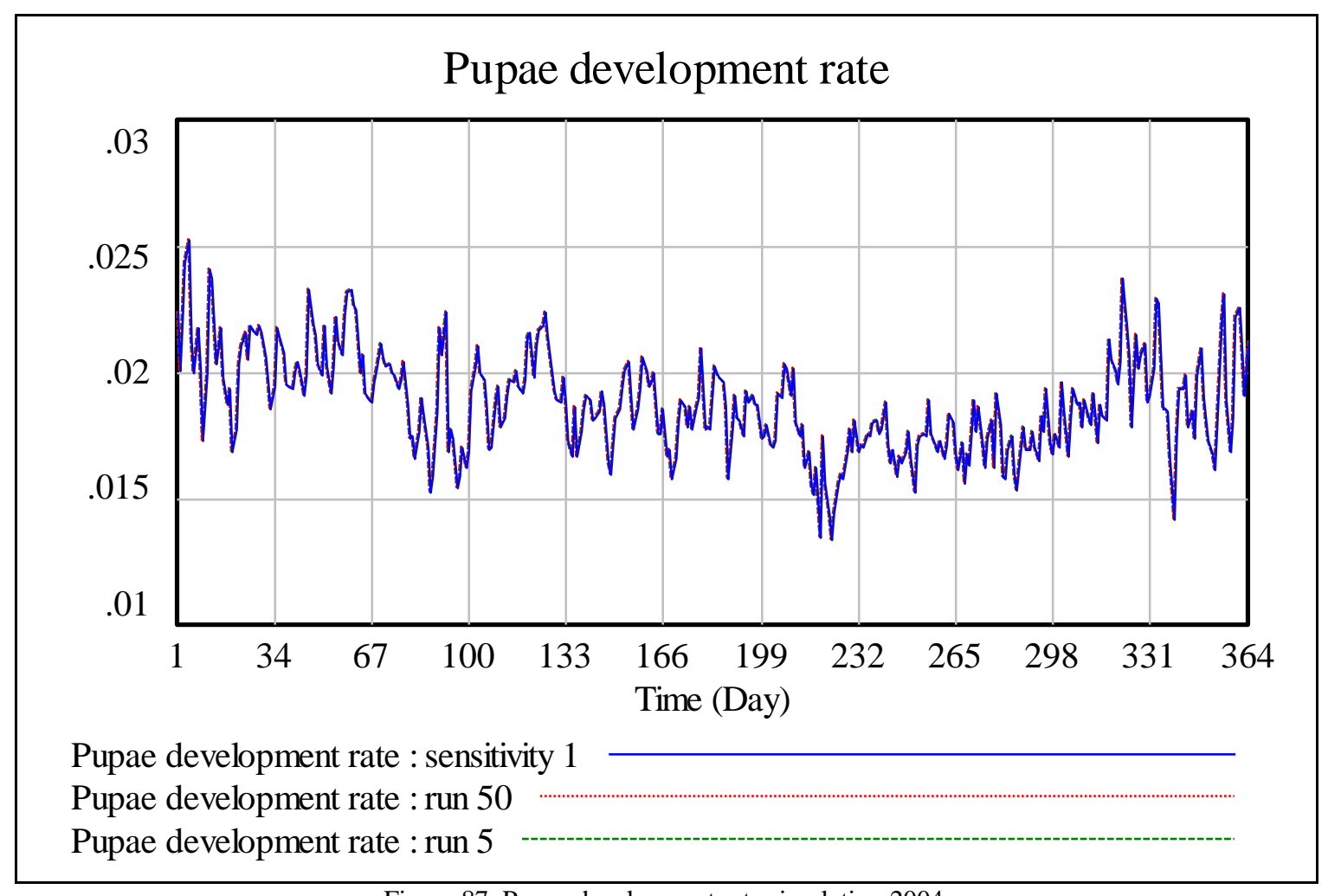

Figure 87: Pupae development rate simulation 2004 


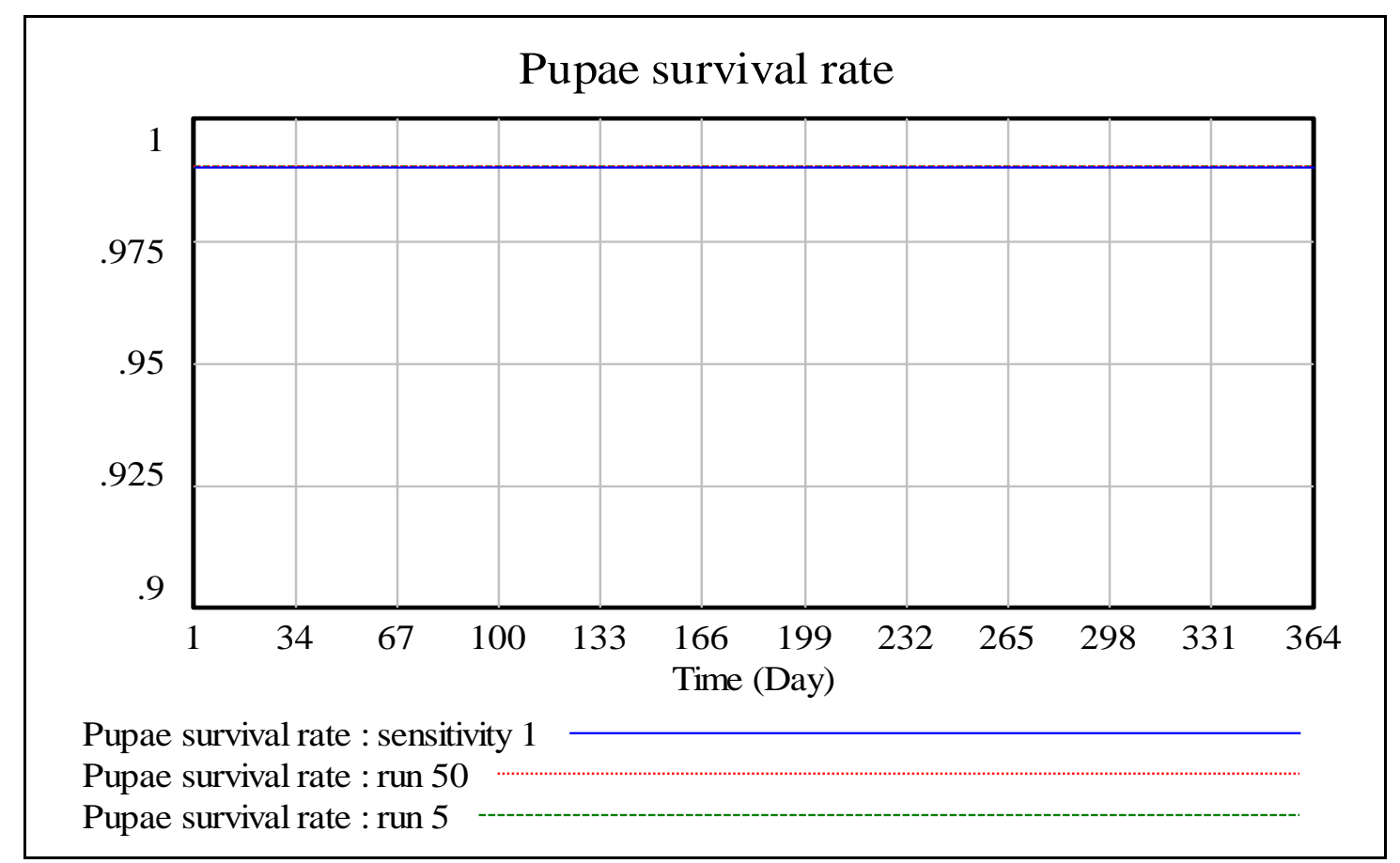

Figure 88: Pupae survival rate simulation 2004

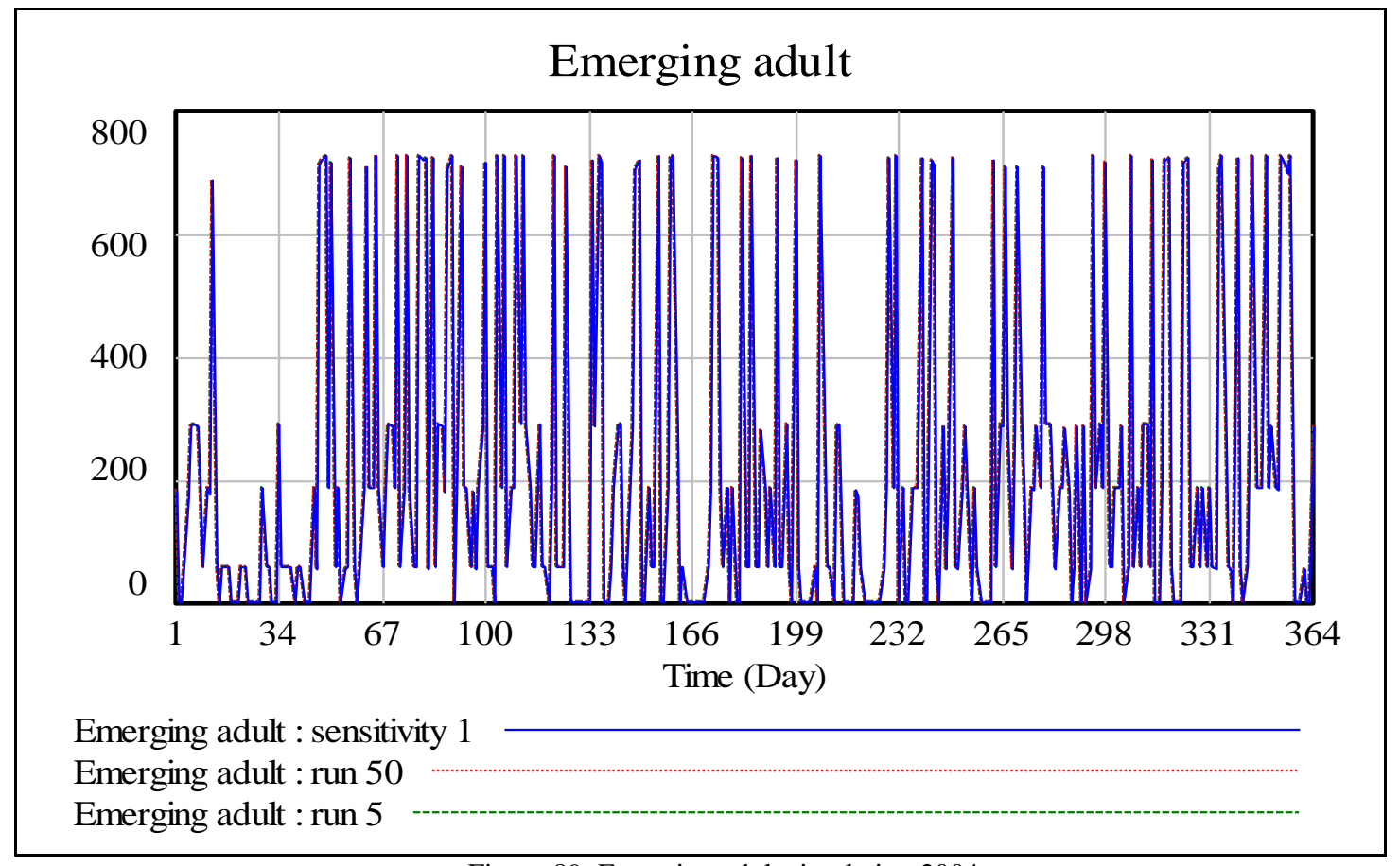

Figure 89: Emerging adult simulation 2004 


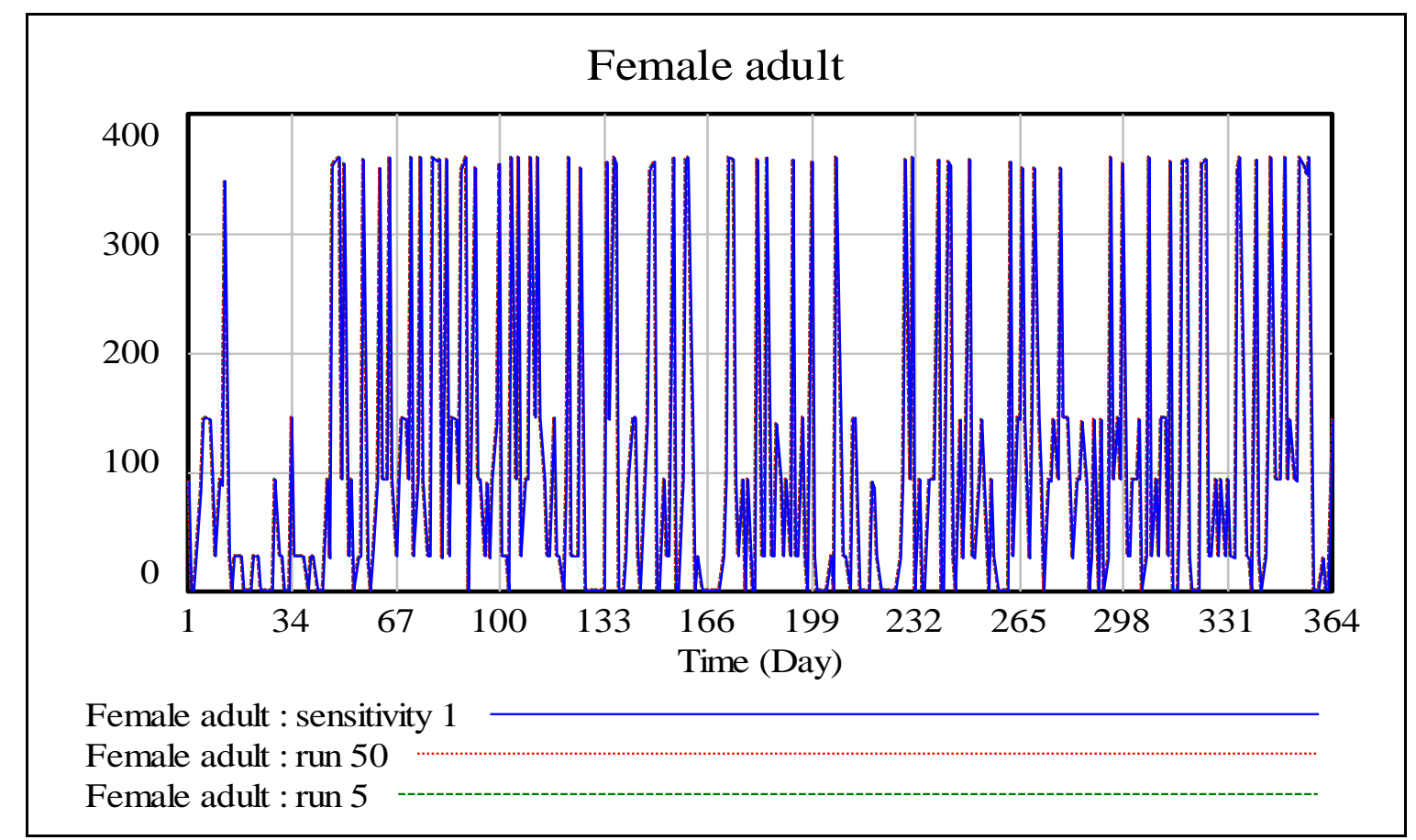

Figure 90: Female adult simulation 2004

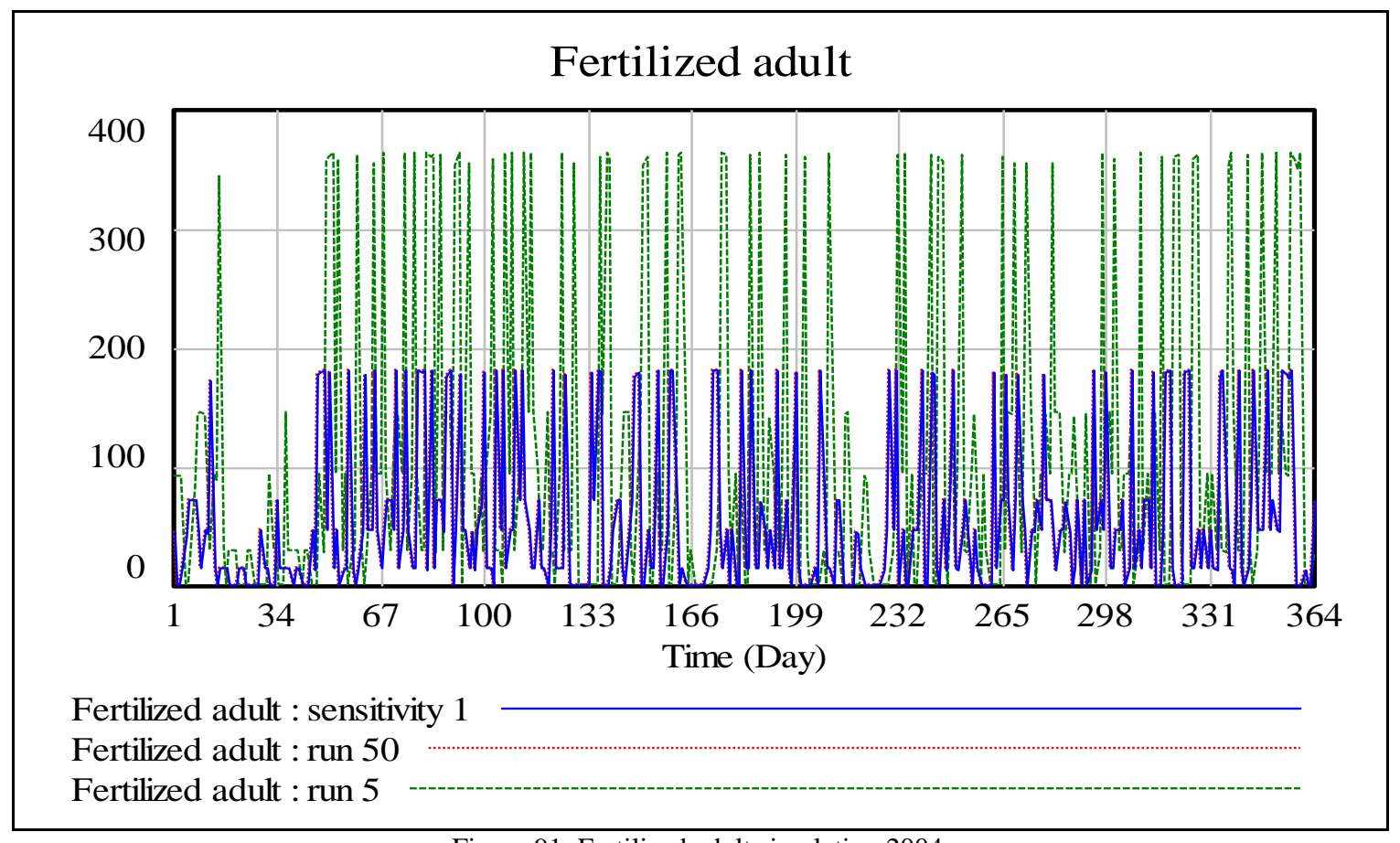

Figure 91: Fertilized adult simulation 2004 


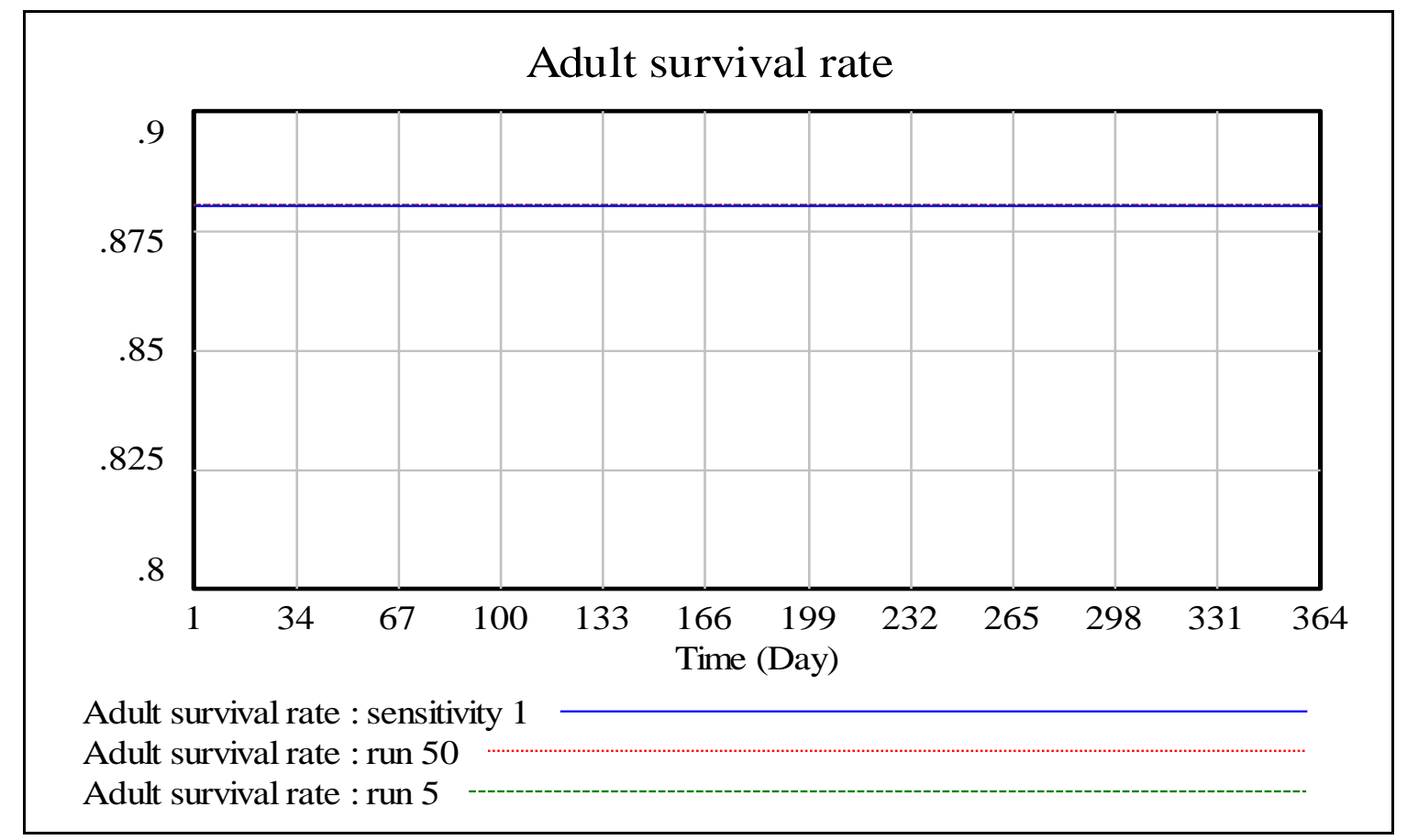

Figure 92: Adult survival rate simulation 2004

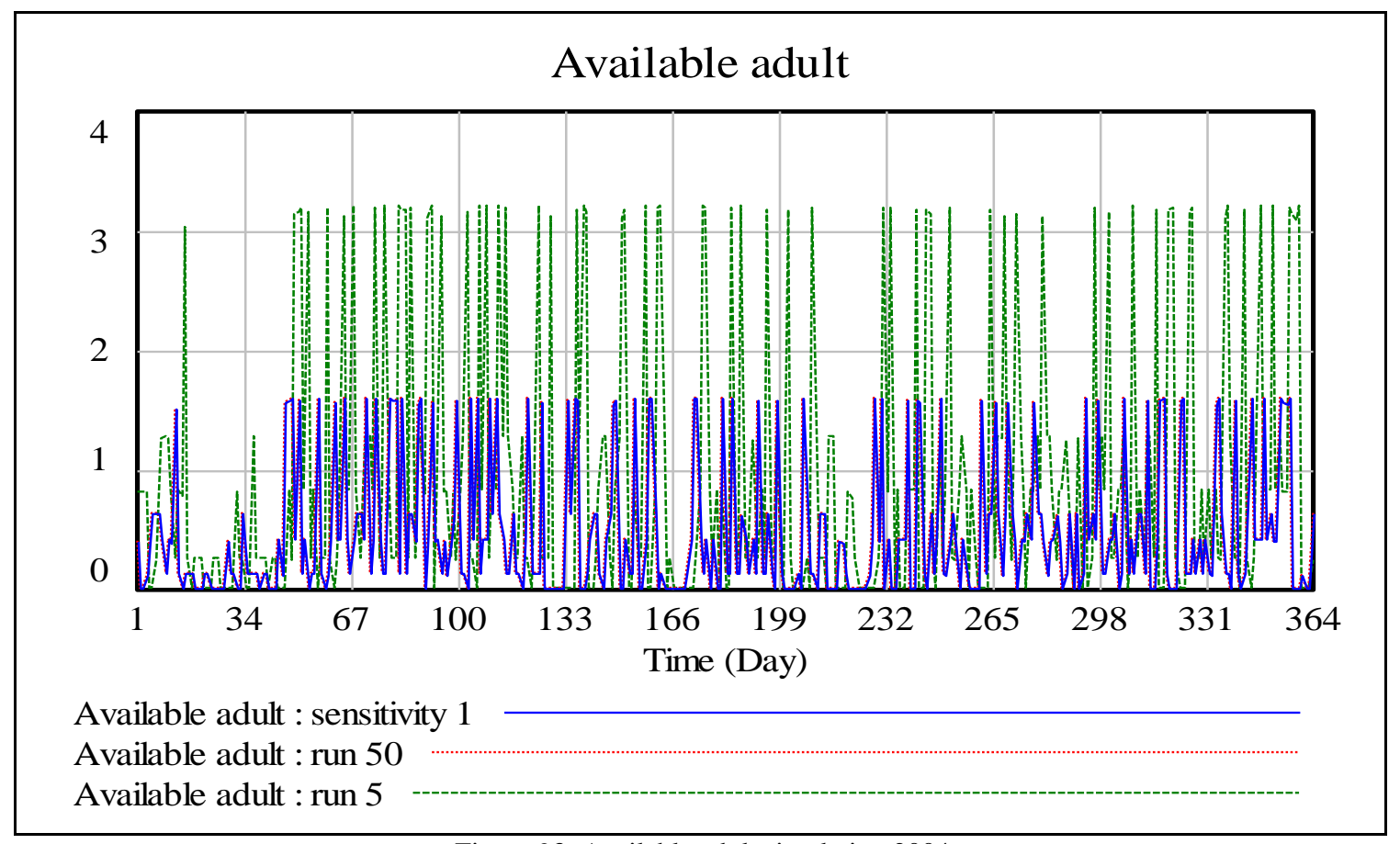

Figure 93: Available adult simulation 2004 


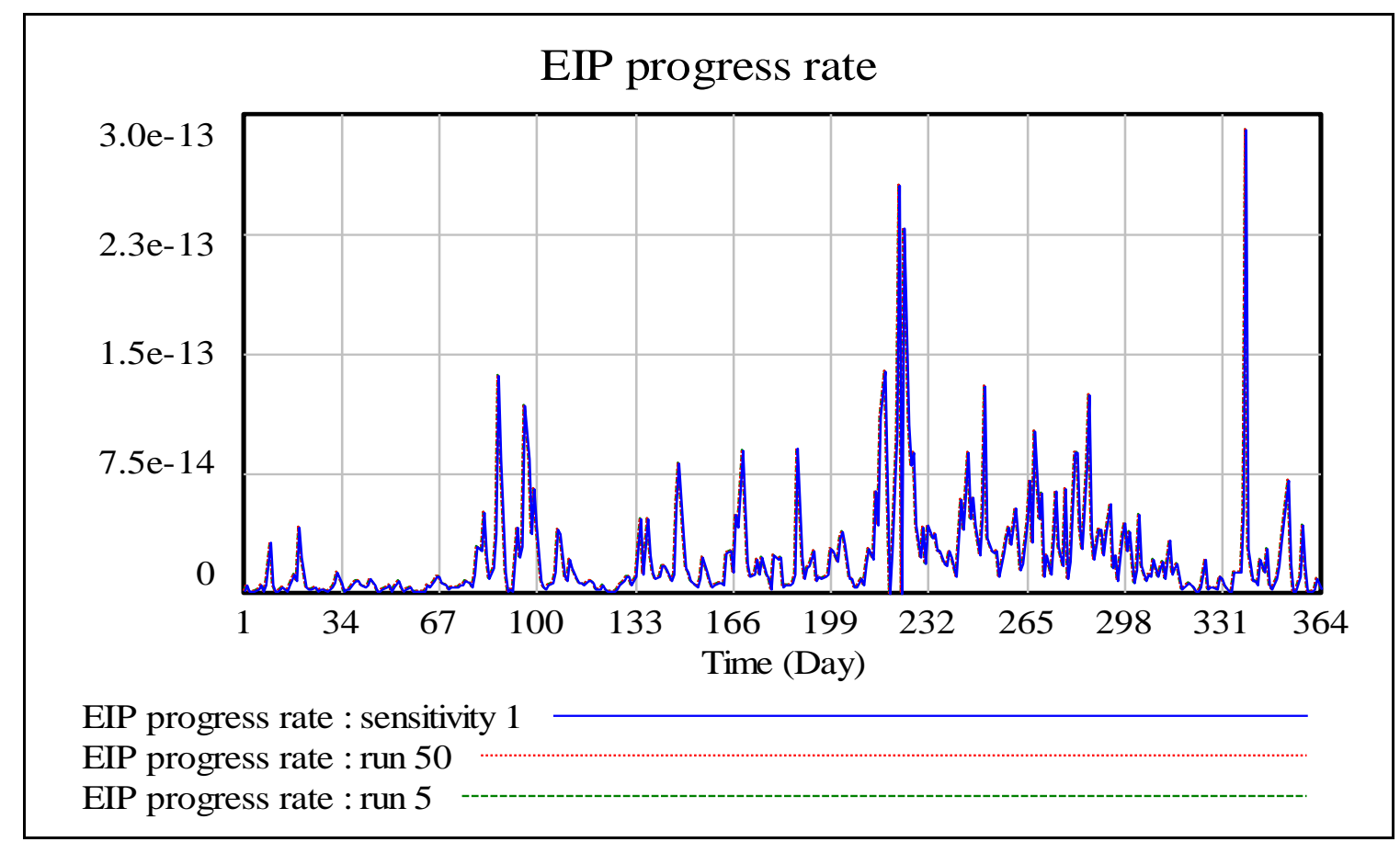

Figure 94: EIP progress rate simulation 2004

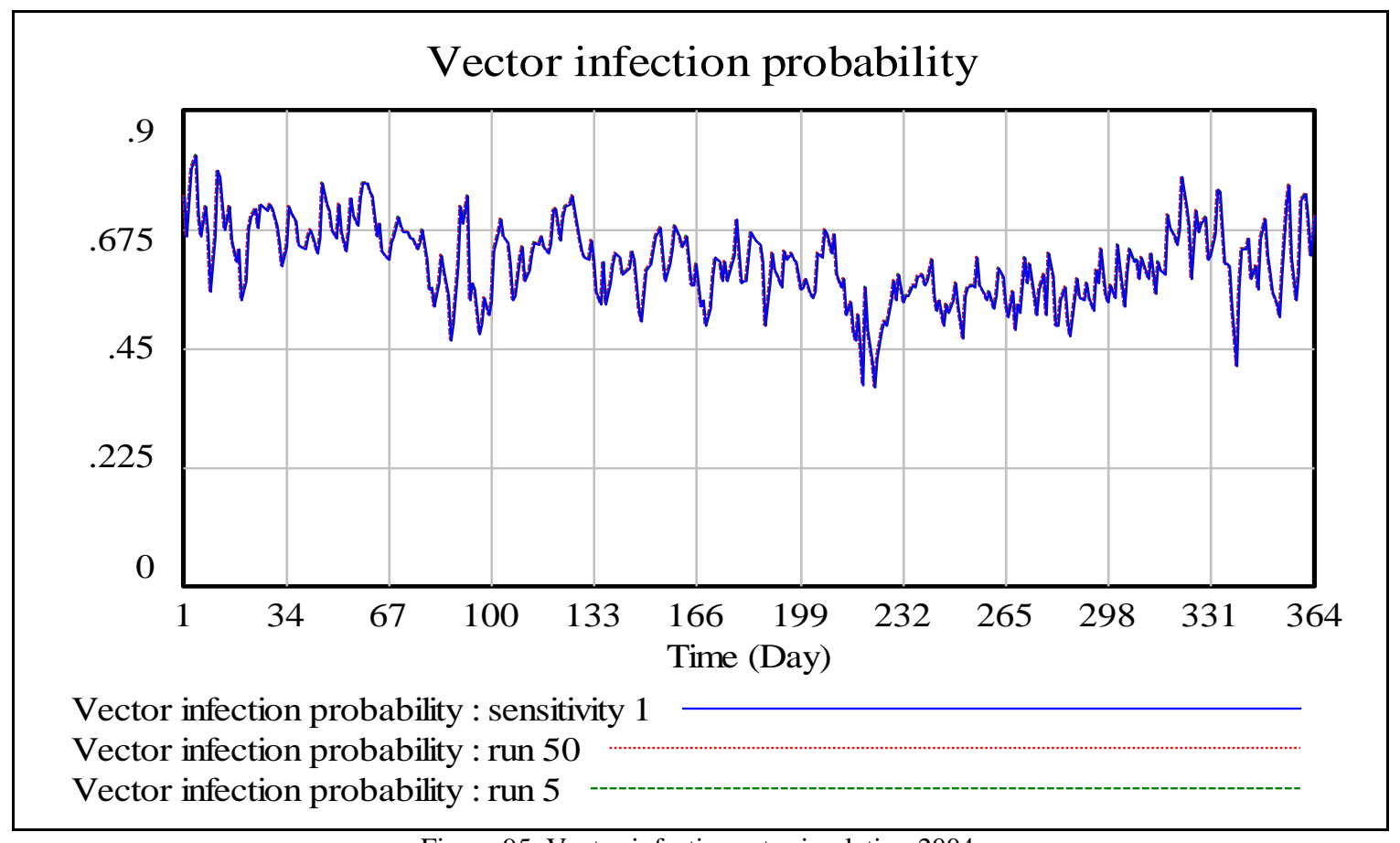

Figure 95: Vector infection rate simulation 2004 


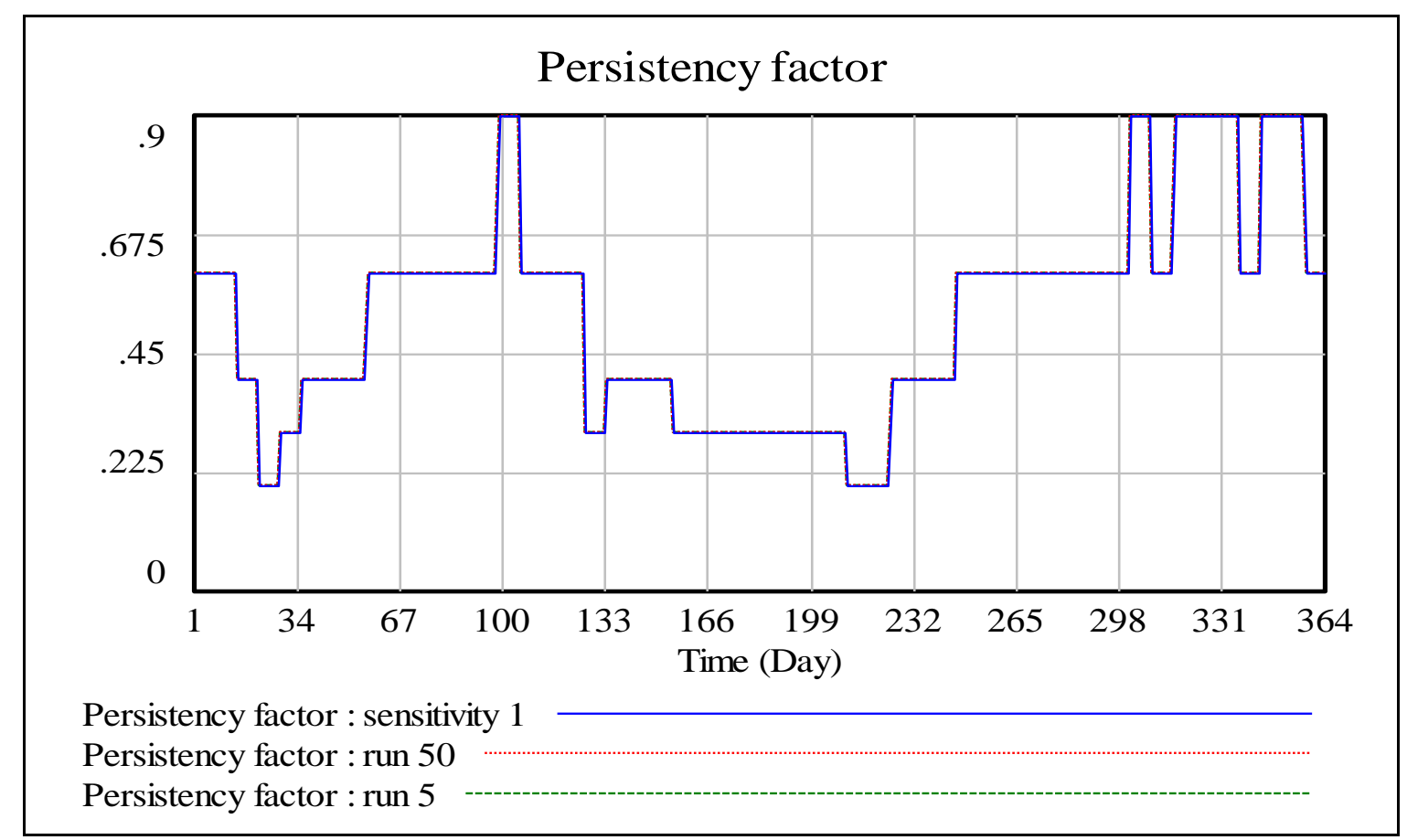

Figure 96: Dew point temperature based persistency factor simulation 2004

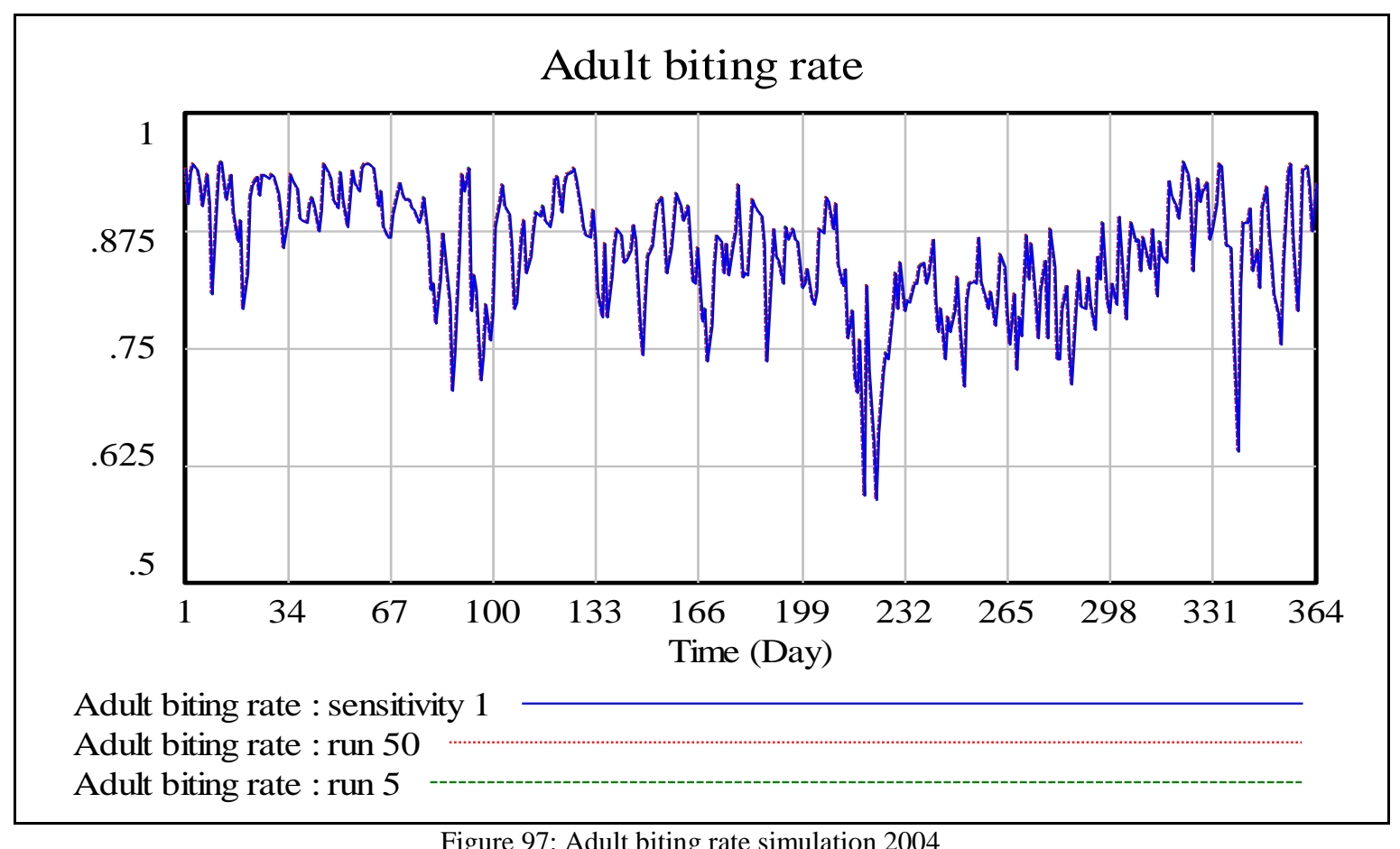

Figure 97: Adult biting rate simulation 2004 


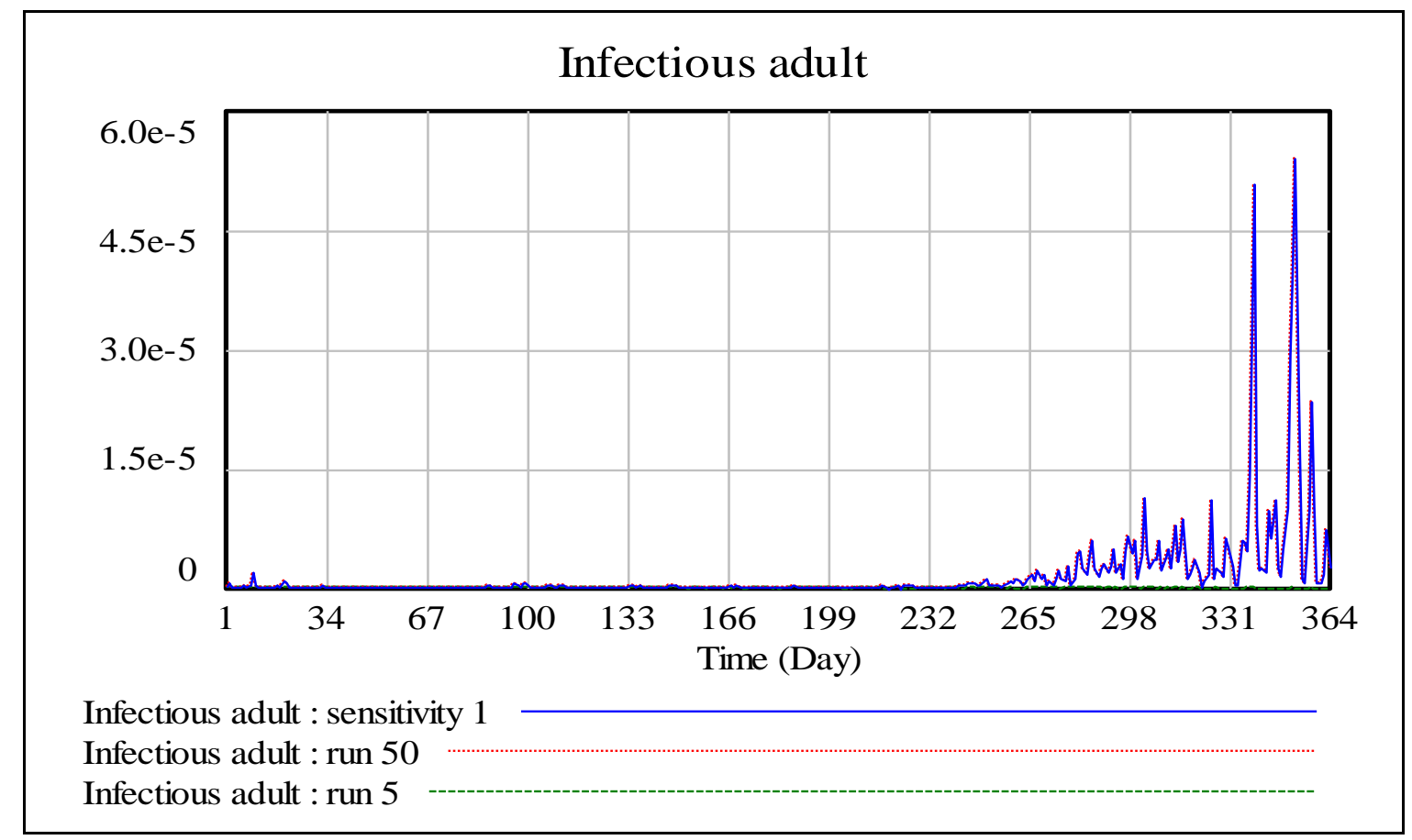

Figure 98: Infectious adult simulation 2004

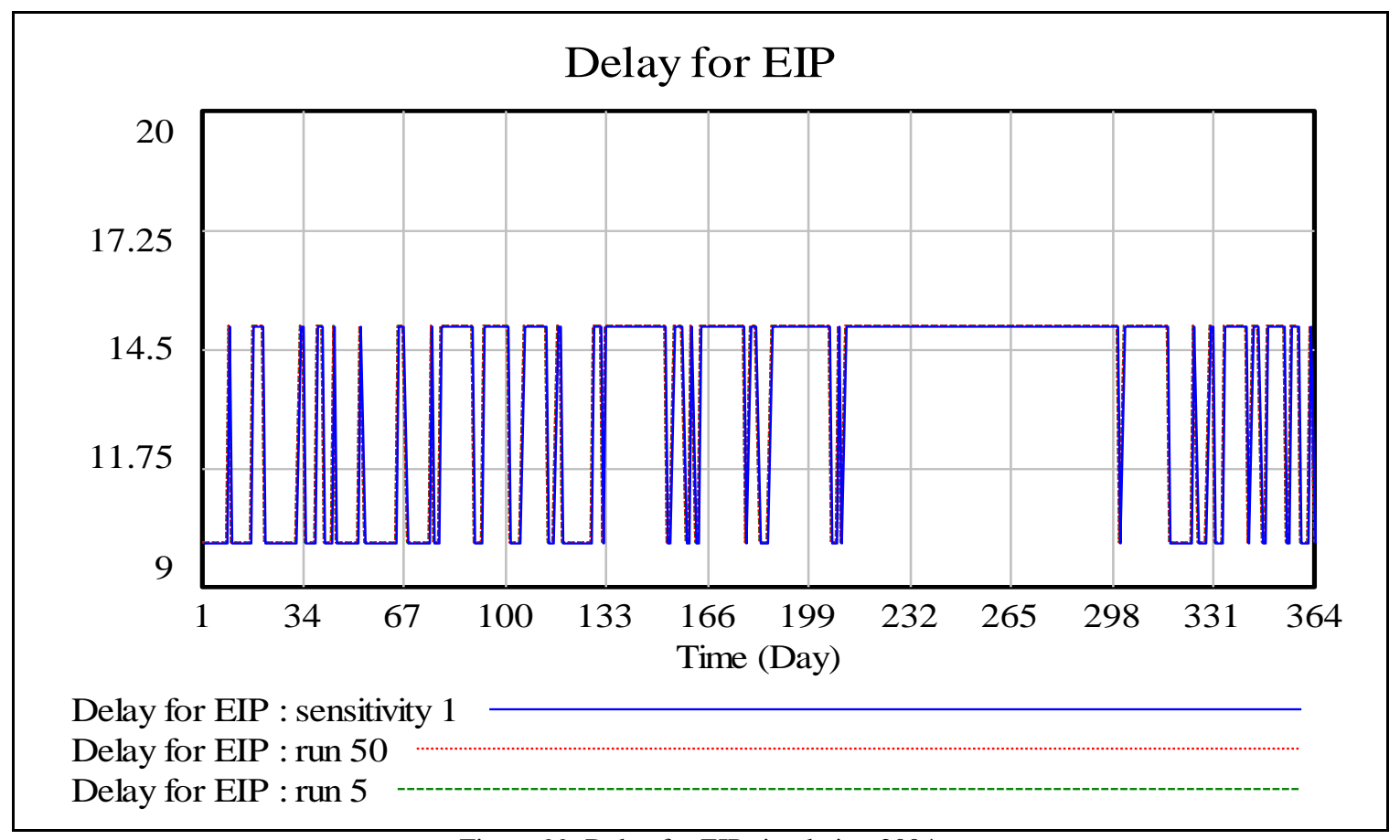

Figure 99: Delay for EIP simulation 2004 


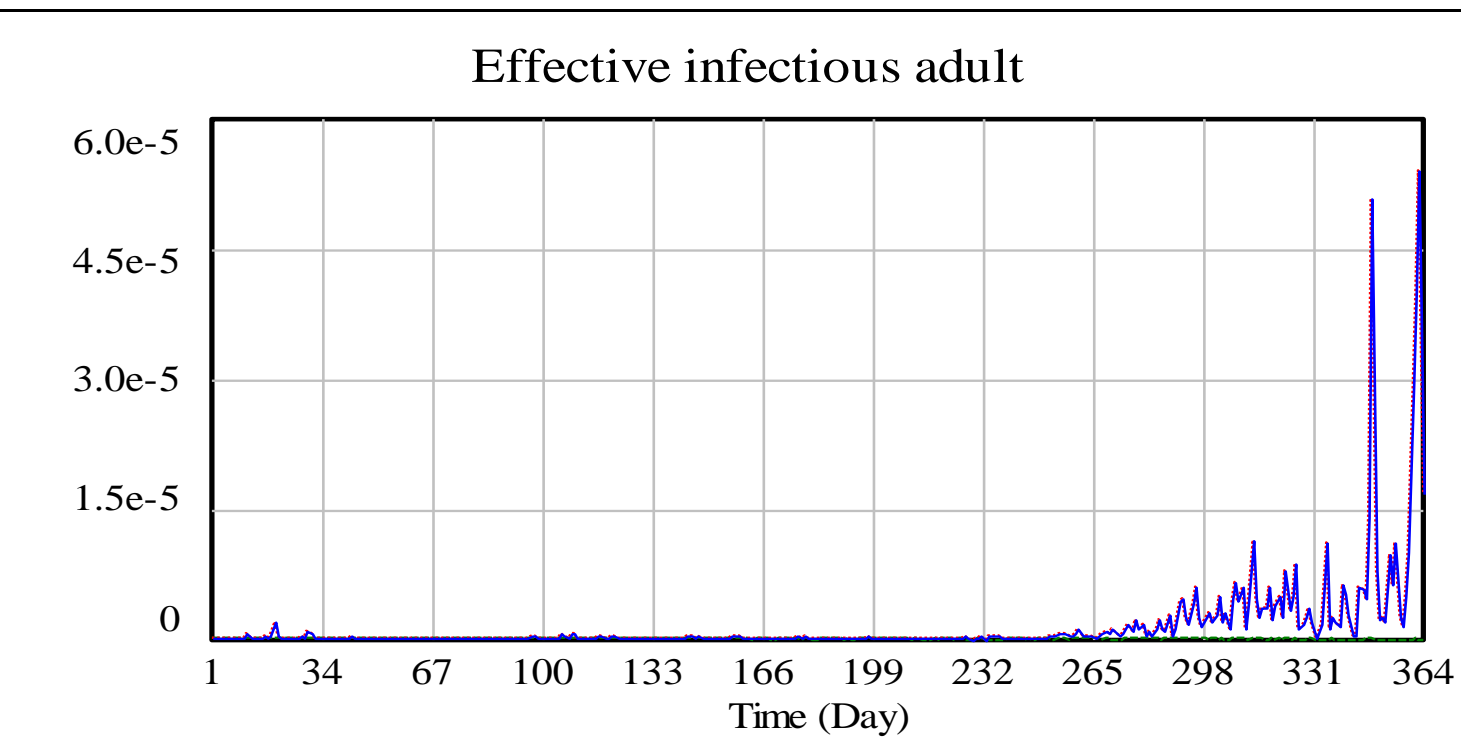

Effective infectious adult : sensitivity 1

Effective infectious adult : run 50

Effective infectious adult : run 5

Figure 100: Effective infectious adult simulation 2004

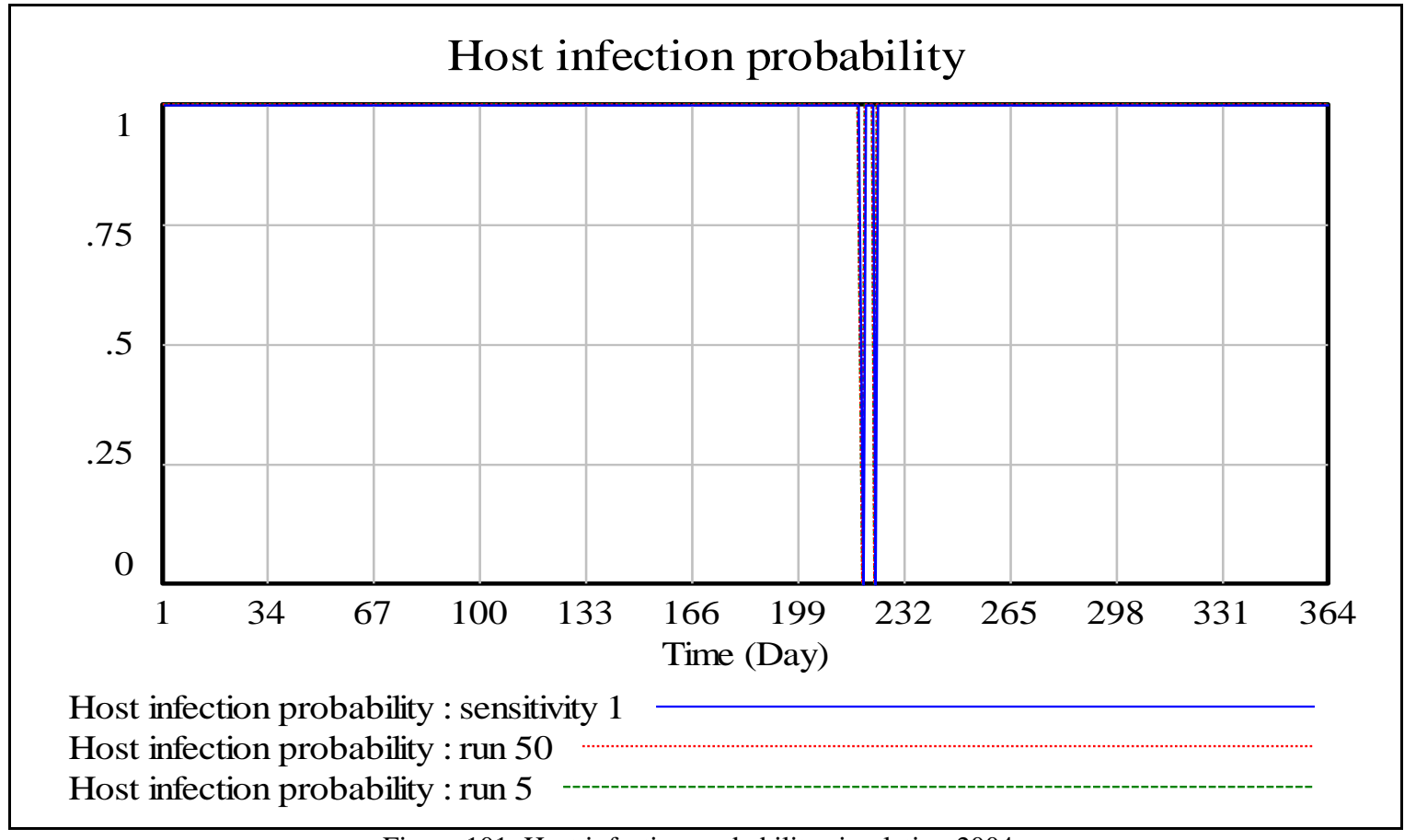

Figure 101: Host infection probability simulation 2004 


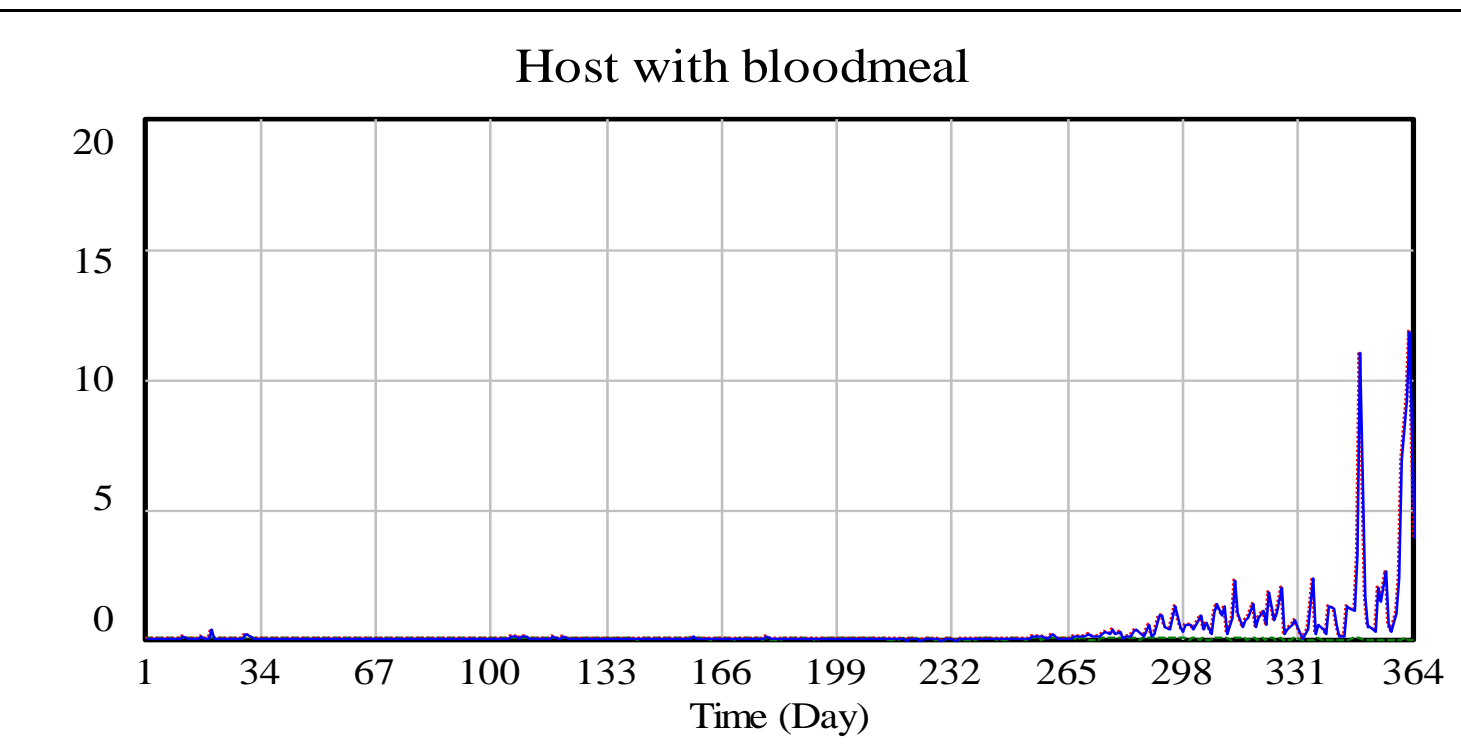

Host with bloodmeal : sensitivity 1

Host with bloodmeal : run 50

Host with bloodmeal : run 5

Figure 102: Host with bloodmeal simulation 2004

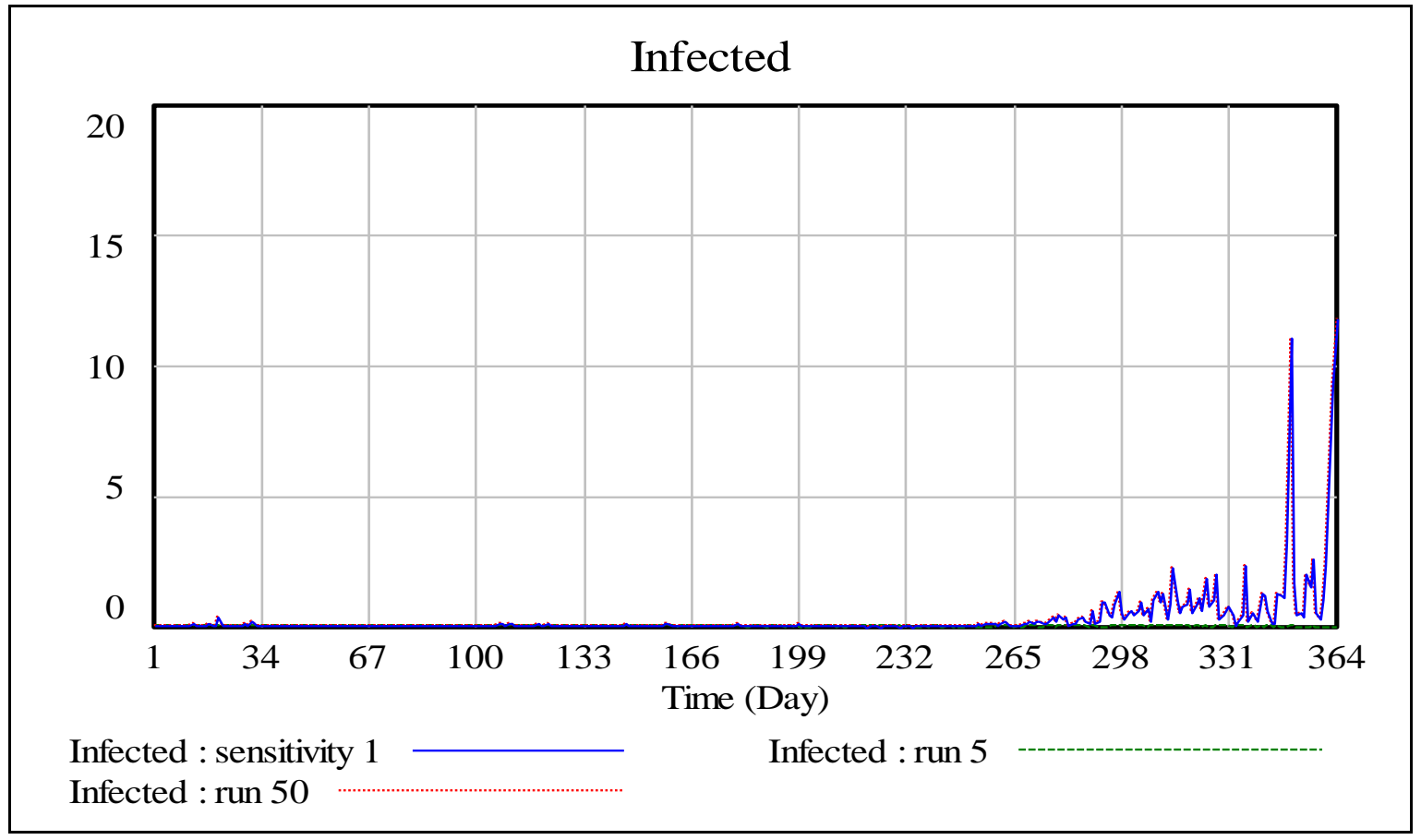

Figure 103: Infected simulation 2004 


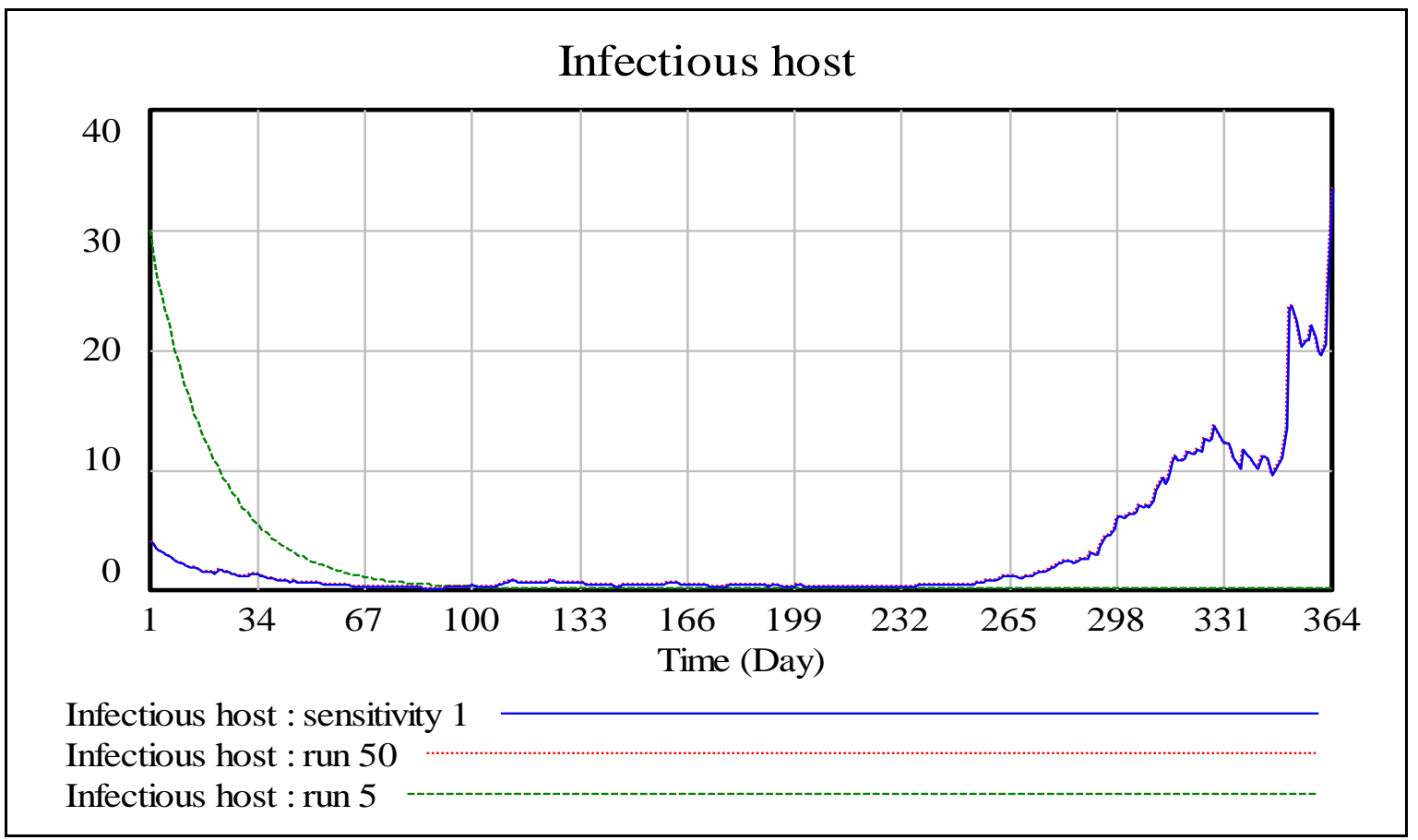

Figure 104: Infectious host simulation 2004 


\section{Bibliography}

Althouse, B. M., Hanley, K. A., Diallo, M., Sall, A. A., Ba, Y., Faye, O., et al. (2015). Impact of Climate and Mosquito Vector Abundance on Sylvatic Arbovirus. The American Society of Tropical Medicine and Hygiene , 92(1), 88-97.

Anderson, R. M., \& May, R. M. (1992). Infectious Diseases of Humans: Dynamics and Control. Oxford: Oxford University Press.

Andraud, M., Hens, N., Marais, C., \& Beutels, P. (2012). Dynamic Epidemiological Models for Dengue Transmission: A Systematic Review of Structural Approaches. PLOS ONE , 7 (11).

Anno, S., Imaoka, K., Tadono, T., Igarashi, T., Sivaganesh, S., Kannathasan, S., et al. (2015). Space-time clustering characteristics of dengue based on ecological, socioeconomic and demographic factors in northern Sri Lanka. Geospat Health, 10 (2).

Araujo, R. V., Albertini, M. R., Costa-da-Silva, A. L., Suesdek, L., Franceschi, N. C., Bastos, N. M., et al. (2015). São Paulo urban heat islands have a higher incidence of dengue than other urban areas. The Brazilian Journal of Infectious Diseases , 19, 146155.

Arboleda, S., Jaramillo, N., \& Peterson, A. T. (2009). Mapping Environmental Dimensions of Dengue Fever Transmission Risk in the Aburrá Valley, Colombia. Int. J. Environ. Res. Public Health , 6, 3040-3055.

Arboleda, S., Jaramillo-O., N., \& Peterson, A. T. (2012). Spatial and temporal dynamics of Aedes aegypti larval sites in Bello, Colombia. Journal of Vector Ecology , 37, 37-48.

Azil, A. H., Long, S. A., Ritchie, S. A., \& Williams, C. R. (2010). The development of predictive tools for pre-emptive dengue vector control: a study of Aedes aegypti abundance and meteorological variables in North Queensland, Australia. Tropical Medicine and International Health, 15 (10), 1190-1197.

Bannister-Tyrrell, M., Williams, C., Ritchie, S. A., Rau, G., Lindesay, J., Mercer, G., et al. (2013). Weather-Driven Variation in Dengue Activity in Australia Examined Using a Process-Based Modeling Approach. Am J Trop Med Hyg , 88, 65-72.

Barbazan, P., M., G., W., B., W., T., D., P., \& J.-P., G. O. (2010). Modelling the effect of temperature on transmission of dengue. Medical and Veterinary Entomology, 24, 66-73.

Bartley, L., Donnelly, C., \& Garnett, G. (2002). The seasonal pattern of dengue in endemic areas: mathematical models of mechanisms. Trans R Soc Trop Med Hyg , 96 (4), 387-397. 
Benthem, B. H., Vanwambeke, S. O., Khantikul, N., Burghoorn-maas, C., Panart, K., Oskam, L., et al. (2005). Spatial Patterns of dengue and risk factors for seropositivity for dengue infection. Am. J. Trop. Med. Hyg., 7 , 72 (2), 201-208.

Bergquist, N. (2001). Vector-borne parasitic diseases: new trends in data collection and risk assessment. Acta Tropica, 79, 13-20.

Brunkard, J. M., Cifuentes, E., \& Rothenberg, S. J. (2008). Assessing the roles of temperature,precipitation, and ENSO in dengue re-emergence on the Texas-Mexico border region. salud pública de méxico , 50 (3), 227-234.

Buczak, A. L., Koshute, P. T., Babin, S. M., Feighner, B. H., \& Lewis, S. H. (2012). A data-driven epidemiological prediction method for dengue outbreaks using local and remote sensing data. BMC Medical Informatics and Decision Making , 12 (124).

Chakravarti, A., \& Kumaria, R. (2005). Eco-epidemiological analysis of dengue infection during an outbreak of dengue fever, India. Virology Journal , 2 (32).

Chan, T.-C., Hu, T.-H., \& Hwang, J.-S. (2015). Daily forecast of dengue fever incidents for urban villages in a city. International Journal of Health Geographics , 14 (9).

Chowell, G., Torre, C. A., Munayco-escate, C., Suarez-ognio, L., Lopez-Cruz, R., Hyman, J. M., et al. (2008). Spatial and temporal dynamics of dengue fever in Peru: 1994-2006. Epidemiol Infect, 136 (12), 1667-1677.

Costa, E. A., Santos, E. M., Correia, J. C., \& Albuquerque, C. M. (2010). Impact of small variations in temperature and humidity on the reproductive activity and survival of Aedes aegypti (Diptera, Culicidae). Revista Brasileira de Entomologia , 54 (3), 488-493.

Curran, P., Atkinson, P., Foody, G., \& EJ, M. (Adv Parasitol). Linking remote sensing, land cover and disease. Am J Epidemiol , 47, 37-80.

D, B., S, D., \& RN, D. (1993). Observations on the breeding habitats of Aedes aegypti in Calcutta following an episode of dengue haemorrhagic fever. Indian Journal of Medical, 97, 44-46.

Depradine, C., \& Lovell, E. (2004). Climatological variables and the incidence of Dengue fever in Barbados. International Journal of Environmental , 14 (6), 429-441.

Descloux, E., Mangeas, M., Menkes, C. E., Lengaigne, M., Leroy, A., Tehei, T., et al. (2012). Climate-Based Models for Understanding and Forecasting Dengue Epidemics. PLoS Negl Trop Dis , 6 (2).

Diekmann, O., \& Heesterbeek, J. (2000). Mathematical epidemiology of infectious diseases:model building, analysis and interpretation (Vol. 5). John Wiley \& Sons. 
DJ, G. (2002). Epidemic dengue/dengue hemorrhagic fever as a public health, social and economic problem in the 21st century. Trends Microbiol , 10 (2), 100-103.

Dom, N. C., Hassan, A. A., Latif, Z. A., \& Ismail, R. (2012). Generating temporal model using climate variables for the prediction of dengue cases in Subang Jaya, Malaysia. Asian Pacific Journal of Tropical Disease , 3, 352-361.

Duncombe, J., Clements, A., Davis, J., Hu, W., \& Ritchie, P. W. (2013). Spatiotemporal patterns of Aedes aegypti populations in Cairns, Australia: assessing drivers of dengue transmission. Tropical Medicine and International Health, 18 (7), 839-849.

Estallo, E. L., Lamfri, M. A., Scavuzzo, C. M., Almeida, F. F., Introini, M. V., Zaidenberg, M., et al. (2008). Models for Predicting Aedes aegypti Larval Indices Based on Satellite Images and Climatic Variables. J. Am. Mosq. Control Assoc. , 24, 368-376.

Esteva, L., \& Vargas, C. (1998). Analysis of a dengue disease transmission model. Mathematical Biosciences, 150 (2), 131-151.

Esteva, L., \& Vargas, C. (2000). Influence of vertical and mechanical transmission on the dynamics of dengue disease. Mathematical Biosciences , 167 (1), 51-64.

Favie, C., Degallier, N., \& Vilarinhos, P. (2006). Effects of climate and different management strategies on Aedes aegypti breeding sites: a longitudinal survey in Brasilia (DF, Brazil). Tropical Medicine and International Health , 11, 1104-1118.

Feng, Z., \& Velasco-Herna'ndez, J. X. (1997). Competitive exclusion in a vector-host model for the dengue fever. J. Math. Biol, 35, 523-544.

Focks, D. A., Brenner, R. J., Hayes, J., \& Daniels, E. (2000). Transmission thresholds for dengue in terms of aedes aegypti pupae per person with discussion of their utility in source reduction efforts. The American Society of Tropical Medicine and Hygiene , 62(1), $11-18$.

Fuller, D. O., Troyo, A., \& Beier, J. C. (2009). El Niño Southern Oscillation and vegetation dynamics as predictors of dengue fever cases in Costa Rica. Environ Res Lett, 4, 140111-140118.

Fuller, D. O., Troyo, A., \& Beier, J. C. (2009). El Niño Southern Oscillation and vegetation dynamics as predictors of dengue fever cases in Costa Rica. Environ Res Lett, 4 (4), 140111-140118.

Fuller, D. O., Troyo, A., Calderón-Arguedas, O., \& Beier, J. C. (2009). Dengue vector (Aedes aegypti) larval habitats in an urban environment of Costa Rica analysed with ASTER and QuickBird imagery. Int. J. Remote Sens. , 31, 3-11. 
Githeko, A. K., Lindsay, S. W., Confalonieri, U. E., \& Patz, J. A. (2000, January). Climate change and vector-borne diseases: a regional analysis. Bulletin of the World Health Organization, 78.

Hales, S., de Wet, N., Maindonald, J., \& Woodward, A. (2002). Potential effect of population and climate changes on global distribution of dengue fever: an empirical model. Lancet, 360, 830-834.

Hay, S. I., Packer, M. J., \& Rogers, D. J. (2010). Review article: The impact of remote sensing on the study and control of invertebrate intermediate hosts and vectors for disease. International Journal of Remote Sensing , 18 (14), 2899-2930.

HM, T., KM, A., \& S., T. (1998). The effect of temperature and humidity on dengue virus propagation in Aedes aegypti mosquitos. Southeast Asian J Trop Med Public Health , 29 (2), 280-284.

Hopp, M. J., \& Foley, J. A. (2001). Global-scale ralelationships between climate and the dengue fever vector, aedes aegypti. Climatic Change , 48, 441-463.

Hu, W., Clements, A., Williams, G., \& Tong, S. (2010). Dengue fever and El Nin o/Southern Oscillation in Queensland, Australia: a time series predictive model. Occup Environ Med , 67, 307-311.

Hurtado-Diaz, M., Riojas-Rodriguez, H., Rothenberg, S. J., Gomez-Dantes, H., \& Cifuentes, E. (2007). Short communication: Impact of climate variability on the incidence of dengue in Mexico. Tropical Medicine and International Health, 12, 1327-1337.

Jansen, N., \& Beebe, C. (2010). The dengue vector Aedes aegypti: what comes next. Microbes and Infection, 22.

Kalluri, S., Gilruth, P., Rogers, D., \& Szczur, M. (2007). Surveillance of Arthropod Vector-Borne Infectious Diseases Using Remote Sensing Techniques: A Review. PLoS Pathog , 3, 1361-1371.

Karl, S., Halder, N., Kelso, J. K., Ritchie, S. A., \& Milne, G. J. (2014). A spatial simulation model for dengue virus infection in urban areas. BMC Infectious Diseases , 14 (447).

Kasperska, E., Kasperski, A., \& Mateja-Losa, E. (2013). Sensitivity Analysis and Optimization on Some Models of Archetypes Using Vensim - Theoretical Issue. Studia Ekonomiczne , 153, 33-52.

Kasperska, E., Mateja-Losa, E., \& Marjasz, R. (2013). Sensitivity Analysis and Optimization for Selected Supply Chain Management Issues in the Company - Using System Dynamics and Vensim. Journal of Entrepreneurship Management and Innovation (JEMI) , 9 (2), 29-44. 
Kasperska, E., Mateja-Losa, E., Bajon, T., \& Marjasz, R. (2014). Did Napoleon Have to Lose the Waterloo Battle?": Some Sensitivity Analysis and Optimization Experiments Using Simulation by Vensim. Studia Ekonomiczne, 188, 97-118.

Kearney, M., P., P. W., Ritchie, S., \& Ary, A. H. (2009). Integrating biophysical models and evolutionary theory to predict climatic impacts on species' ranges: the dengue mosquito Aedes aegypti in Australia. Functional Ecology, 23, 528-538.

Khalid, B., \& Ghaffar, A. (2015). Dengue transmission based on urban environmental gradients in different cities of Pakistan. Int J Biometeorol, 59, 267-283.

Khalid, B., \& Ghaffar, A. (2015). Dengue transmission based on urban environmental gradients in different cities of Pakistan. International Journal of Biometeorology , 59 (3), 267-283.

Limper, M., Thai, K. T., Gerstenbluth, I., Osterhaus, A. D., Duits, A. J., \& Gorp, E. C. (2014). Climate Factors as Important Determinants of Dengue Incidence in Curacao. Zoonoses and Public Health .

Lin, Y., Kaihu, H., \& Jinyuan, Z. (2012). Research on the Optimization of Retailer Inventory Strategy based on System Dynamics Simulation. Service Systems and Service Management (ICSSSM). Shanghai: IEEE.

Ljung, L. (1999). System Identification: Theory for the User, 2nd Edition . New Jersey: IEEE Robotics \& Automation Magazine.

Lowe, R., TrevorC.Bailey, DavidB.Stephenson, RichardJ.Graham, CaioA.S.Coelho, Carvalho, M. S., et al. (2011). Spatio-temporalmodellingofclimatesensitivediseaserisk:Towards an early warning system for dengue in Brazil. Computers \&Geosciences , 37, 371-381.

Lu, L., Lin, H., Tian, L., Yang, W., Sun, J., \& Liu, Q. (2009). Time series analysis of dengue fever and weather in Guangzhou, China. BMC Public Health, 9 (395).

Luz, P. M., Mendes, B. V., Codeço, C. T., Struchiner, C. J., \& Galvani, A. P. (2008). Time Series Analysis of Dengue Incidence in Rio de Janeiro, Brazil. Am J Trop Med Hyg , 79 (6), 933-939.

Machault, V., Yébakima, A., Etienne, M., Vignolles, C., Palany, P., Tourre, Y. M., et al. (2014). Mapping Entomological Dengue Risk Levels in Martinique Using HighResolution Remote-Sensing Environmental Data. ISPRS Int. J. Geo-Inf , 3 (4), 13521371.

Maidana, N. A., \& Yang, H. M. (2008). Describing the geographic spread of dengue disease by traveling waves. Mathematical Biosciences , 215 (1), 64-77. 
Massad, E., Ortega, N. R., Struchiner, C. J., \& Burattini, M. N. (2003). Fuzzy epidemics. Artificial intelligence in medicine , 29 (3), 241-259.

Mohammed, A., \& Chadee, D. D. (2011). Effects of different temperature regimens on the development of Aedes aegypti (L.) (Diptera: Culicidae) mosquitoes. Acta Tropica, 119, 38-43.

Montoya, Y., Holechek, S., Caceres, O., Palacios, A., Burans, J., Guevara, C., et al. (2003). Circulation of Dengue Viruses in North-Western Peru, 2000-2001. Dengue Bulletin , 27, 52-62.

Morin, C. W., Comrie, A. C., \& Ernst, K. (2013). Climate and Dengue Transmission: Evidence and Implications. Environmental Health Perspectives , 121, 11-12.

Morrison, A. C., Minnick, S. L., Rocha, C., Forshey, B. M., Stoddard, S. T., Getis, A., et al. (2010). Epidemiology of Dengue Virus in Iquitos, Peru 1999 to 2005: Interepidemic and Epidemic Patterns of Transmission. Neglected Tropical Diseases .

Murray, J. (2003). Geographic spread and control of epidemics. Mathematical Biology,II: Spatial Models and Biomedical Applications, Springer, 661-721.

Neteler, M., Roiz, D., Rocchini, D., Castellani, C., \& Rizzoli, A. (2011). Terra and Aqua satellites track tiger mosquito invasion: modelling the potential distribution of Aedes albopictus in north-eastern Italy. International Journal of Health Geographics , 10.

Newman, M. E. (2002). The spread of epidemic disease on networks. Phys. Rev. E, 66.

Newton, E. A., \& Reiter, P. (1992). A model of the transmission of dengue fever with an evaluation of the impact of ultra-low volume (ULV) insecticide applications on dengue epidemics. Am J Trop Med Hyg , 47 (6), 709-720.

Nishiura, H. (2006). Mathematical and Statistical Analyses of the Spread of Dengue. Dengue Bulletin , 30, 51-67.

O'Gower, A. (1956). Control Measures for Aedes aegypti. Surveys in Northern Australia. Health , 6, 40-42.

Olson, J. A. (2006). Climate change and health: global to local. Annals of Tropical Medicine \& Parasitology , 100, 535-549.

Organization, W. H. (2009). Dengue: guidelines for diagnosis, treatment,prevention and control.

Otero, M., \& Solari, H. (2010). Stochastic eco-epidemiological model of dengue disease transmission by Aedes aegypti mosquito. Mathematical Biosciences , 223 (1), 32-46. 
Otero, M., Solari, H. G., \& Schweigmann, N. (2006). A Stochastic Population Dynamics Model for Aedes Aegypti: Formulation and Application to a City with Temperate Climate. Bulletin of Mathematical Biology, 68 (8), 1945-1974.

Peterson, A. T., Martínez-Campos, C., Nakazawa, Y., \& Martínez-Meyer, E. (2005). Time-specific ecological niche modeling predicts spatial dynamics of vector insects and human dengue cases. Trans R Soc Trop Med Hyg , 99 (9), 647-655.

Pongsumpun, P., \& Tang, I. (2001). A realistic age structured transmission model for dengue hemorrhagic fever in Thailand. Southeast Asian J Trop Med Public Health, 32 (2), 336-340.

Rohani, A., Wong, Y., Zamre, I., Lee, H., \& Zurainee, M. (2009). The effect of extrinsic incubation temperature on development of dengue serotype 2 and 4 viruses in Aedes Aegypti. Southeast Asian Journal of Tropical Medicine and Public Health, 40, 942-950.

Roiz, D., Neteler, M., Castellani, C., Arnoldi, D., \& Rizzoli, A. (2011). Climatic Factors Driving Invasion of the Tiger Mosquito (Aedes albopictus) into New Areas of Trentino, Northern Italy. PLoS One , 6.

Rotela, C., Fouque, F., Lamfri, M., Sabatier, P., Introini, V., Zaidenberg, M., et al. (2007). Space-time analysis of the dengue spreading dynamics in the 2004 Tartagal outbreak, Northern Argentina. Acta Tropica, 103 (1), 1-13.

Sang, S., Gu, S., Bi, P., Yang, W., Yang, Z., Xu, L., et al. (2014). Predicting Unprecedented Dengue Outbreak Using Imported Cases and Climatic Factors in Guangzhou, 2014. PLoS Negl Trop Dis , 9 (5).

Sarfraz, M. S., Tripathi, N. K., Tipdecho, T., Thongbu, T., Kerdthong, P., \& Souris, M. (2012). Analyzing the spatio-temporal relationship between dengue vector larval density and land-use using factor analysis and spatial ring mapping. BMC Public Health, 12.

SB, H. (2008). Dengue virus-mosquito interactions. Anпи Rev Entomol , 53, 273-291.

Shope, R. (1991). Global climate change and infectious diseases. Environ Health Perspect , 96, 171-174.

Side, S., \& Noorani, S. M. (2013). A SIR Model for Spread of Dengue Fever Disease (Simulation for South Sulawesi, Indonesia and Selangor, Malaysia). World Journal of Modelling and Simulation, 9 (2), 96-105.

Stefani, A., Dusfour, I., Corrêa, A. P., Cruz, M. C., Dessay, N., Galardo, A. K., et al. (2013). Land cover, land use and malaria in the Amazon: a systematic literature review of studies using remotely sensed data. Malaria Journal, 12 (192). 
Torres, C., Barguil, S., Melgarejo, M., \& Olarte, A. (2014). Fuzzy model identification of dengue epidemic in Colombia based on multiresolution analysis. Artificial Intelligence in Medicine, 60, 41-51.

Tran, A., \& Raffy, M. (2006). On the dynamics of dengue epidemics from large-scale information. Theoretical Population Biology, 69 (1), 3-12.

Troyo, A., Fuller, D. O., Calderón-Arguedas, O., Solano, M. E., \& Beier, J. C. (2009). Urban structure and dengue fever in Puntarenas, Costa Rica. Singap J Trop Geogr. , 30 (2), 265-282.

Vaidya, A., Bravo-Salgado, A. D., \& Mikler, A. R. (2014). Modeling climate-dependent population dynamics of mosquitoes to guide public health policies. 5th ACM Conference on Bioinformatics,Computational Biology, and Health Informatics (pp. 380-389). Newport Beach, California: ACM.

Vanwambeke, S. O., Bennett, S. N., \& Kapan, D. D. (2011). Spatially disaggregated disease transmission risk: land cover, land use and risk of dengue transmission on the island of Oahu. Trop Med Int Health. , 16 (2), 174-185.

Vezzani, D., Velázquez, S. M., \& Schweigmann, N. (2004). Seasonal pattern of abundance of Aedes aegypti (Diptera: Culicidae) in Buenos Aires City, Argentina. Memórias do Instituto Oswaldo Cruz, 99 (4), 351-356.

Wu, P.-C., Guoa, H.-R., Lung, S.-C., Lin, C.-Y., \& Sua, H.-J. (2007). Weather as an effective predictor for occurrence of dengue fever in Taiwan. Acta Tropica, 103, 50-57.

Xu, H.-Y., Fu, X., Lee, L. K., Ma, S., Goh, K. T., Wong, J., et al. (2014). Statistical Modeling Reveals the Effect of Absolute Humidity on Dengue in Singapore. Plos Neglected Tropical Diseases, 8 (5).

Yang, G.-J., Vounatsou, P., Xiao-Nong, Z., Utzinger, J., \& Tanner, M. (2005). A review of geographic information system and remote sensing with applications to the epidemiology and control of schistosomiasis in China. Acta Tropica , 96, 117-129.

Yu, H.-L., Yang, S.-J., Yen, H.-J., \& Christakos, G. (2011). A spatio-temporal climatebased model of early dengue fever warning in southern Taiwan. Stoch Environ Res Risk Assess , 25, 485-494. 